\title{
Bio-inspired Water-Driven Catalytic Enantioselective Protonation
}

Si Joon Park,${ }^{\dagger}$ In-Soo Hwang, ${ }^{\dagger}$ Young Jun Chang, ${ }^{\dagger}$ Choong Eui Song*

†These authors contributed equally.

*Corresponding author. E-mail: s1673@skku.edu

\section{Supporting Information}




\section{Table of Contents}

$\begin{array}{ll}\text { General Information } & \text { S3 }\end{array}$

$\begin{array}{ll}\text { Synthetic Procedures and Analytical Data } & \text { S5 }\end{array}$

Catalyst preparation $\quad$ S5

General procedure for the enantioselective protonation reaction under various conditions

$\begin{array}{lr}\text { Supplementary Tables } & \text { S14 }\end{array}$

Table S1. Screening of hydrophobic cosolvents $\quad$ S14

Table S2. Equivalent screening $\quad$ S15

Table S3. Effect of droplet size under the biphasic microfluidic conditions $\quad$ S16

Table S4. High-pressure experiments for the determination of activation volume $\quad$ S17

Table S5. Study of the effect of catalyst optical purity on product optical purity $\quad$ S18

$\begin{array}{lr}\text { Supplementary Figures } & \text { S19 }\end{array}$

Figure S1. Thiol screening $\quad$ S19

Figure S2. Schematic representation of the double-subtraction procedure of IR spectra

S20

Figure S3. Isotope experiment with $\left[1-{ }^{2} \mathrm{H}\right]$-phenylglyoxal $\quad$ S21

Figure S4. Study of the rate determining step for isomerization of hemithioacetals $\quad$ S22

$\begin{array}{ll}\text { NMR Spectra } & \text { S23 }\end{array}$

$\begin{array}{ll}\text { NMR Spectra of catalysts } & \text { S23 }\end{array}$

NMR Spectra of protonation products $\quad$ S25

$\begin{array}{ll}\text { HPLC Spectra } & \text { S46 }\end{array}$

HPLC Spectra of Scheme 1B $\quad$ S46

HPLC Spectra of Table S1 $\quad$ S51

HPLC Spectra of Table S2 $\quad$ S64

HPLC Spectra of Scheme 1C/Figure S1 $\quad$ S68

$\begin{array}{ll}\text { HPLC Spectra of Scheme 2C } & \text { S75 }\end{array}$

HPLC Spectra of Scheme $3 \quad$ S81

References

S111 


\section{General Information}

\section{Chemicals}

Chemicals were purchased from various companies (including Acros, Aldrich, TCI, Alfa Aesar and Fluka) as reagent grade and used without further purification. The glyoxals 1a, 1e-1g and $1 \mathrm{~m}$ were obtained as a hydrate commercially and used without further purification. The glyoxals 1b-1d, 1h-11, 1n and 1o were prepared from the corresponding ketone ${ }^{1}$ or aldehyde ${ }^{2}$ according to the literature procedure.

\section{Solvents}

Dried solvents (including $\mathrm{CH}_{2} \mathrm{Cl}_{2}$, THF, toluene, and xylenes) were purchased from various companies (including Merck, Aldrich, and Alfa Aesar) and used without further purification. Additional solvents were purchased from various commercial suppliers.

\section{Inert Gas}

Dry argon was purchased from Hanmi Gas with > 99.99\% purity.

\section{Glassware}

All non-aqueous reactions were performed in flame-dried glassware under Ar. Solvents were removed under reduced pressure at $30{ }^{\circ} \mathrm{C}$ using a rotary evaporator and were dried under high vacuum $\left(10^{-1} \mathrm{mbar}\right)$.

\section{Thin Layer Chromatography}

Thin-layer chromatography (TLC) was performed using silica gel plates (Merck, Kieselgel 60 F254 $0.25 \mathrm{~mm}$ ).

\section{Column chromatography}

Column chromatography was carried out using Merck silica gel (60 A, 230-400 mesh, particle size $0.040-0.063 \mathrm{~mm}$ ) with technical grade solvents. Elution was accelerated using compressed air.

\section{Nuclear Magnetic Resonance Spectroscopy}

${ }^{1} \mathrm{H},{ }^{13} \mathrm{C}$ and ${ }^{19} \mathrm{~F}$ nuclear magnetic resonance (NMR) spectra were recorded on a Bruker Ascend ${ }^{\mathrm{TM}}$ 500 spectrometer in a suitable deuterated solvent. The solvent employed and the respective measuring frequency are indicated for each experiment. Chemical shifts are reported with tetramethylsilane (TMS) serving as an internal reference for ${ }^{1} \mathrm{H}$ and ${ }^{13} \mathrm{C}$ NMR analysis and with two or one digits after the comma. The resonance multiplicity is described as s (singlet), $d$ (doublet), t (triplet), q (quartet), $\mathrm{m}$ (multiplet), and bs (broad singlet). All spectra were recorded at $298 \mathrm{~K}$ unless otherwise noted and processed with the MestReNova 6.0.2 suites of programs, and the coupling constants are reported as observed. The residual deuterated solvent signal relative to tetramethylsilane was used as the internal reference in the ${ }^{1} \mathrm{H}$ NMR spectra $\left(\mathrm{CDCl}_{3} \delta\right.$ 7.26), and are reported as follows: chemical shift $\delta$ in ppm (multiplicity, coupling constant $J$ in $\mathrm{Hz}$, number of protons). ${ }^{13} \mathrm{C}$ NMR spectra are reported in ppm from tetramethylsilane (TMS), with the solvent resonance as the internal standard $\left(\mathrm{CDCl}_{3} \delta\right.$ 77.2). All spectra are broadband decoupled unless otherwise noted. ${ }^{19} \mathrm{~F}$ NMR $(470.4 \mathrm{MHz})$ spectra were recorded using a Bruker 
Ascend ${ }^{\mathrm{TM}} 500$ spectrometer with benzotrifluoride $\left(\mathrm{C}_{6} \mathrm{H}_{5} \mathrm{CF}_{3}\right)$ as the external standard. All spectra are broadband decoupled unless otherwise noted.

Mass Spectrometry

High-resolution mass spectrometry (HRMS) was recorded using a Bruker Compact mass spectrometer.

Infrared Spectroscopy

Infrared (IR) spectroscopy was performed using a Bruker Vertex 70 spectrometer with the MIRacle Micro ATR accessory.

\section{Specific Rotations}

Specific rotations $\left([\alpha]_{\mathrm{D}}{ }^{\mathrm{T}}\right)$ were measured with a PerkinElmer Polarimeter 343 Plus at room temperature with a sodium lamp (sodium D line, $\lambda=589 \mathrm{~nm}$ ).

\section{High Performance Liquid Chromatography}

High performance liquid chromatography (HPLC) was performed on a YL9100 Plus HPLC System instrument equipped with an isostatic pump using a chiral column (CHIRALPAK AD-H, CHIRALCEL OD-H, CHIRALCEL OX-3, CHIRALPAK IA, CHIRALPAK IC; $250 \times 4.6$ mm).

\section{Magnetic stirrer}

The stirring rate (rpm) was controlled using a CORNING PC-420D.

\section{Syringe Pump}

The injection speed (flow rate) was controlled with a KD Scientific Legato 200 syringe pump.

\section{Tubing}

FEP tubing was used (250, 500, 750 and $1000 \mu \mathrm{m}$ ID). 


\section{Synthetic procedures and analytical data}

\section{Catalyst preparation}

Catalysts were prepared according to the published procedures. ${ }^{3}$

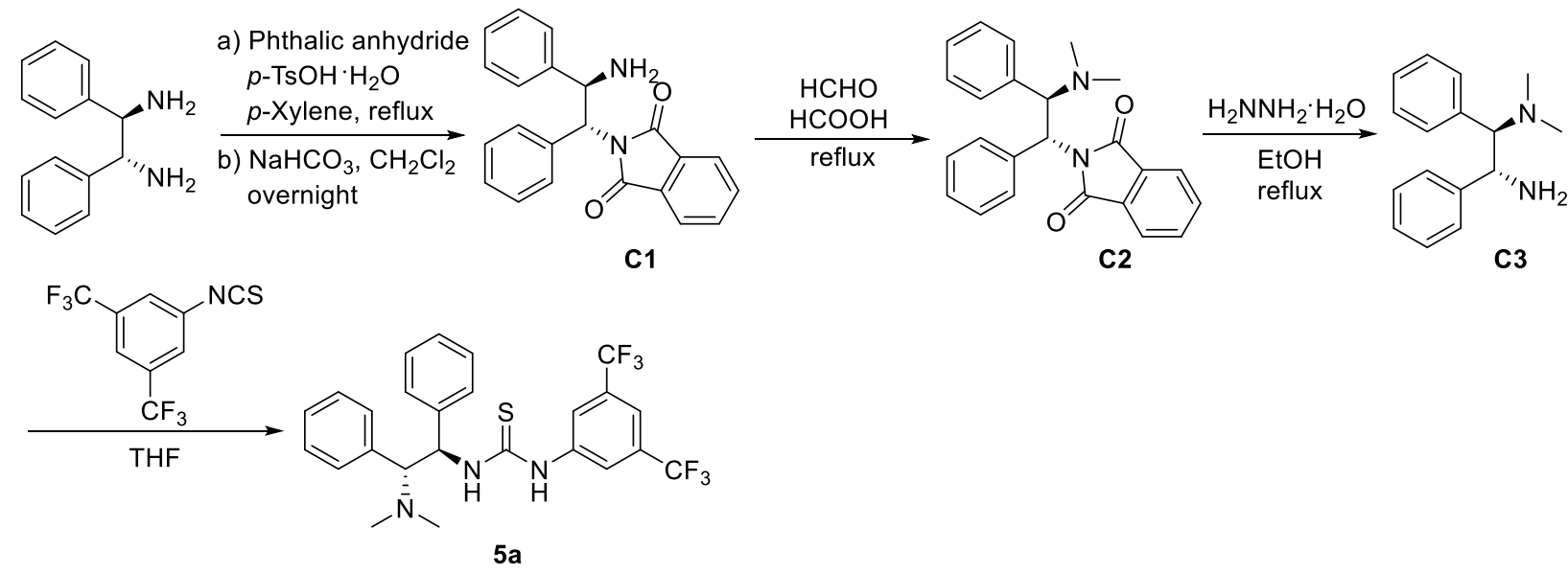

Preparation of C1: $p$-TsOH $\cdot \mathrm{H}_{2} \mathrm{O}(3.8 \mathrm{~g}, 20 \mathrm{mmol}, 1$ equiv) was dissolved in $p$-xylene (50 mL). The reaction mixture was stirred at $60{ }^{\circ} \mathrm{C}$ for 30 minutes, which generated a pink solution. After cooling to room temperature, $(1 R, 2 R)-(+)-1,2$-diphenylethylenediamine $(4.25 \mathrm{~g}, 20 \mathrm{mmol}, 1$ equiv) was added to the solution followed by phthalic anhydride ( $2.96 \mathrm{~g}, 20 \mathrm{mmol}, 1$ equiv). The reaction mixture was stirred under reflux for 3 hours. After the solution was cooled to room temperature, the colorless solid was collected by filtration and washed with 1:1 mixture of $p$ xylene and hexane. The solid was then dried under vacuum for 30 minutes. Subsequently, DCM $(50 \mathrm{~mL})$ and saturated $\mathrm{NaHCO}_{3}$ (aq.) $(32 \mathrm{~mL})$ were added, and the solution was stirred overnight. The organic layer was separated, washed with brine, and dried over anhydrous $\mathrm{Na}_{2} \mathrm{SO}_{4}$. The solvent was evaporated in vacuo to give product $\mathbf{C 1}$ as a white solid (5.53 g, $81 \%$ yield), which was used without further purification.

Preparation of C2: A solution of C1 $(5.53 \mathrm{~g}, 16.1 \mathrm{mmol})$ in $\mathrm{HCHO}(37 \%$ aq., $12.1 \mathrm{~mL})$ and $\mathrm{HCOOH}(80 \%$ aq., $9.7 \mathrm{~mL})$ was stirred under reflux for 12 hours. After cooling to room temperature, saturated $\mathrm{Na}_{2} \mathrm{CO}_{3}$ (aq.) $(30 \mathrm{~mL})$ was added dropwise at $0{ }^{\circ} \mathrm{C}$. The solution was extracted with DCM, and dried over anhydrous $\mathrm{Na}_{2} \mathrm{SO}_{4}$. The reaction mixture was concentrated in vacuo, and purified by silica gel column chromatography (hexanes/EtOAc $=1: 4$ ) to yield a yellow solid (5.56 g, $92 \%$ yield).

Preparation of C3: To a solution of C2 (5.56 g, $15 \mathrm{mmol})$ in ethanol (80 $\mathrm{mL})$, hydrazine monohydrate $(2.2 \mathrm{~mL}, 45 \mathrm{mmol}, 3$ equiv) was added dropwise. The mixture was stirred under reflux for 6 hours. After the solution was cooled to room temperature, it was diluted with diethyl ether and filtered to remove the precipitate. The filtrate was washed with DI water twice to remove excess hydrazine. The organic layer was dried over $\mathrm{Na}_{2} \mathrm{SO}_{4}$ and concentrated in vacuo. C3 was obtained as a yellow solid (2.95 g, 82\% yield), which was used without further purification. 
Preparation of 5a: In a flame-dried RBF, C3 $(2.4 \mathrm{~g}, 10 \mathrm{mmol})$ was dissolved in THF (anhydrous, $25 \mathrm{~mL}$ ), and cooled down to $0{ }^{\circ} \mathrm{C}$. Subsequently, the corresponding isothiocyanate (13 mmol, 1.3 equiv) was added to the reaction mixture dropwise, which was then stirred at RT for 24 hours. The reaction mixture was concentrated in vacuo, and purified by silica gel column chromatography (hexanes/acetone $=1: 8)$ to yield a white solid $(4.757 \mathrm{~g}, 93 \%$ yield $)$.

Catalyst 5a: white solid<smiles>[R16][R16]N(C)[C@H](c1ccccc1)[C@@H](NC(=S)Nc1cc(C(F)(F)F)cc(C(F)(F)F)c1)c1ccccc1</smiles>

${ }^{1} \mathrm{H}$ NMR $\left(500 \mathrm{MHz}, \mathrm{CDCl}_{3}\right): \delta 8.45$ (br s, 2H), $7.84-7.62(\mathrm{~m}$, $2 \mathrm{H}), 7.29-7.02(\mathrm{~m}, 10 \mathrm{H}), 5.42(\mathrm{br} \mathrm{s}, 1 \mathrm{H}), 3.83(\mathrm{~d}, J=11.0 \mathrm{~Hz}$, $2 \mathrm{H}), 2.20(\mathrm{~s}, 6 \mathrm{H}) ;{ }^{13} \mathrm{C} \mathrm{NMR}\left(125 \mathrm{MHz}, \mathrm{CDCl}_{3}\right): \delta 180.47$, $139.58,139.17,132.56\left(\mathrm{q},{ }^{2} J_{\mathrm{C}-\mathrm{F}}=33.57 \mathrm{~Hz}\right), 131.34,129.91$, $128.54,128.07,127.95,127.84,123.75,122.93\left(\mathrm{q},{ }^{1} J_{\mathrm{C}-\mathrm{F}}=\right.$ $273.00 \mathrm{~Hz}), 119.03,73.94,59.43,40.52$.

The analytical data was identical to the reported value. ${ }^{3}$

Catalyst 5b: New compound, white solid; mp: $95^{\circ} \mathrm{C}$<smiles>COc1ccc(C(c2ccc(OC)cc2)[C@H](NC(=S)Nc2cc(C(F)(F)F)cc(C(F)(F)F)c2)N(C)C)cc1</smiles>

$(S, S)-5 b$

${ }^{1} \mathrm{H}$ NMR (500 MHz, $\left.\mathrm{CDCl}_{3}\right): \delta 8.21$ (br s, 2H), $7.73(\mathrm{~s}$, 2H), $7.65(\mathrm{~s}, 1 \mathrm{H}), 7.07(\mathrm{~d}, J=8.2 \mathrm{~Hz}, 2 \mathrm{H}), 6.97(\mathrm{~d}, J=$ $8.4 \mathrm{~Hz}, 2 \mathrm{H}), 6.79(\mathrm{~d}, J=8.7 \mathrm{~Hz}, 2 \mathrm{H}), 6.72(\mathrm{~d}, J=8.0 \mathrm{~Hz}$, 2H), 5.14 (s, 1H), 3.77 (s, 3H), 3.71 (s, 3H), 2.19 (s, 6H); ${ }^{13} \mathrm{C}$ NMR $\left(125 \mathrm{MHz}, \mathrm{CDCl}_{3}\right): \delta 159.17,130.94,128.96$, $123.41,122.94\left(\mathrm{q},{ }^{1} J_{\mathrm{C}-\mathrm{F}}=272.8 \mathrm{~Hz}\right), 118.77,114.23$, $113.32,73.53,59.08,55.14,40.44$.

IR (neat): $v 1611,1512,1469,1382,1277,1249,1177$, 1134, 1037.

HRMS (m/z, ESI): $[\mathrm{M}+\mathrm{H}]^{+}$cald. for $\mathrm{C}_{27} \mathrm{H}_{28} \mathrm{~F}_{6} \mathrm{~N}_{3} \mathrm{O}_{2} \mathrm{~S}, 572.1807$; found, 572.1803 . 


\section{General procedure for the enantioselective protonation reaction under various conditions}

Under on-water batch conditions: The corresponding thiol 2 ( $1 \mathrm{mmol}, 10$ equiv) was added to a mixture of glyoxal $1(0.1 \mathrm{mmol})$, catalyst 5 (0.03 mmol, $30 \mathrm{~mol} \%)$, eucalyptol (1 mmol, 10 equiv) and brine $(2.0 \mathrm{~mL})$. The reaction mixture was stirred vigorously with a magnetic bar at $1150 \mathrm{rpm}$ and the temperature was set to $20{ }^{\circ} \mathrm{C}$. After completion of the reaction $(96 \mathrm{~h})$, the reaction mixture was extracted with EtOAc $(3 \times 5 \mathrm{~mL})$. The combined organic layers were washed with brine, dried over $\mathrm{Na}_{2} \mathrm{SO}_{4}$, filtered, and concentrated in vacuo. The residue was purified by column chromatography on silica gel eluting with a hexanes/ethyl acetate mixture, affording the desired product 4.

Under on-water microfluidic conditions: The organic solutions (solution 1: in-situ-generated hemithioacetal $3(0.2 \mathrm{mmol})$ made by heating a mixture of phenylglyoxal $\mathbf{1}$ and thiol $\mathbf{2}$ (10 equiv) in eucalyptol $(16.7 \mathrm{~mL})$; solution 2 : catalyst 5a $(0.06 \mathrm{mmol}, 30 \mathrm{~mol} \%)$ in eucalyptol $(16.7 \mathrm{~mL})$ ) and the aqueous solution (brine, $5.13 \mathrm{M}$ )) were loaded into separate syringes, then injected onto the static mixing connector. The brine and organic solutions were brought together at the mixing connector, then coflowed and compartmentalized into droplets by flow focusing of the aqueous phase with the organic phase. After the tube was filled with the reaction mixture, the flows of brine and organic solutions were stopped, and the outlet of the tube was then sealed tightly with a paraffin film. The other end of the tube was also sealed with a paraffin film. The biphasic plugs were kept inside the FEP tubing at $20{ }^{\circ} \mathrm{C}$ without any shaking. After $96 \mathrm{~h}$, the reaction mixture was collected from the outlet of the tubing by flushing with argon. The organic phase was then purified by chromatography to determine the ee value $(92 \% e e)$. Conversion of the reaction mixture was determined using ${ }^{1} \mathrm{H}$ NMR spectroscopy by comparing the product peak integration with the reactant peak integration.

Typical high-pressure experimental procedure: Phenylglyoxal 1a (15.2mg, $0.1 \mathrm{mmol}$ ), tertiary butyl thiol $\mathbf{2 d}(112 \mu \mathrm{L}, 1 \mathrm{mmol})$, catalyst $5 \mathbf{a}(15.3 \mathrm{mg}, 0.03 \mathrm{mmol})$ and anhydrous eucalyptol or dichloromethane $(2 \mathrm{~mL})$ were added to a $4 \mathrm{~mL}$ Teflon tube at $20{ }^{\circ} \mathrm{C}$ and the tube was capped with exclusion of air. This tube was placed in a high-pressure reactor vessel $(1.5 \mathrm{~cm}$ inner diameter; $12 \mathrm{~cm}$ vessel diameter; $27.5 \mathrm{~cm}$ height) (see photographs below). The cylinder was filled with water and the vessel was pressurized with a hydraulic press to subject the Teflon tube to the necessary pressure. After a specific time had elapsed, the pressure was removed by pulling up the ram of the hydraulic press, and the Teflon tube was recovered from the vessel. The reaction mixture was directly used to measure conversion by ${ }^{1} \mathrm{H} \mathrm{NMR}$.

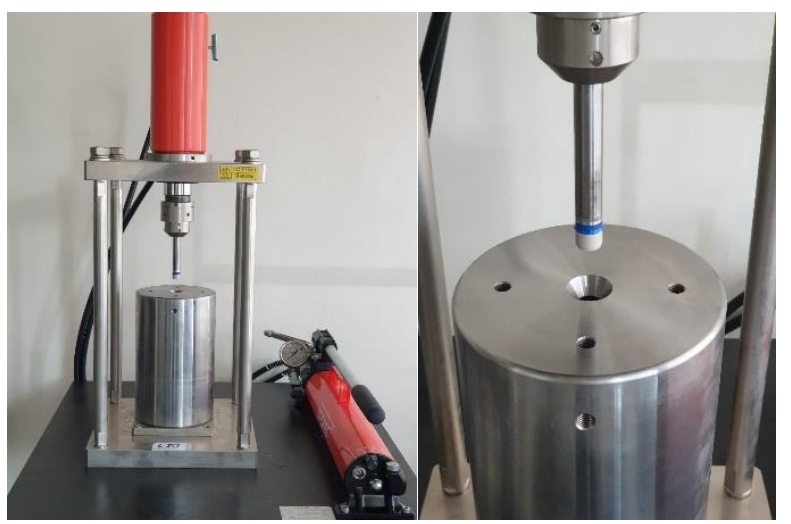

Hydraulic press and high-pressure reactor vessel for high pressure experiments 
Compound 4aa: Isolated yield: $24.1 \mathrm{mg}(93 \%)$; colorless liquid

${ }^{1} \mathrm{H}$ NMR $\left(500 \mathrm{MHz}, \mathrm{CDCl}_{3}\right): \delta 7.41-7.32(\mathrm{~m}, 5 \mathrm{H}), 7.28-7.20(\mathrm{~m}$,<smiles>O=C(SCc1ccccc1)C(O)c1ccccc1</smiles>

$(R)-\mathbf{4 a a}$

$5 \mathrm{H}), 5.20(\mathrm{~d}, J=4.4 \mathrm{~Hz}, 1 \mathrm{H}), 4.10(\mathrm{dd}, J=34.2,13.7 \mathrm{~Hz}, 2 \mathrm{H}), 3.52(\mathrm{~d}$, $J=4.4 \mathrm{~Hz}, 1 \mathrm{H}) ;{ }^{13} \mathrm{C}$ NMR $\left(125 \mathrm{MHz}, \mathrm{CDCl}_{3}\right): \delta 201.38,137.93$, 136.72, 128.99, 128.91, 128.84, 128.69, 127.49, 127.13, 79.91, 33.36. HPLC analysis: Chiralpak AD-H, Hex/IPA = 96/4, $1.0 \mathrm{~mL} / \mathrm{min}, 220$ $\mathrm{nm} ; \mathrm{t}_{R}=33.3 \min ($ major, $R$ ), 27.0 min (minor, $S$ )

The analytical data was identical to the reported value. ${ }^{4}$

Compound 4ab: Isolated yield: $15.4 \mathrm{mg}(74 \%)$; colorless liquid<smiles>CCCSC(=O)C(O)c1ccccc1</smiles>

$(R)-4 \mathbf{a b}$

${ }^{1} \mathrm{H}$ NMR $\left(500 \mathrm{MHz}, \mathrm{CDCl}_{3}\right): \delta 7.50-7.32(\mathrm{~m}, 5 \mathrm{H}), 5.22(\mathrm{~d}, J=4.4 \mathrm{~Hz}$, $1 \mathrm{H}), 3.66-3.50(\mathrm{~m}, 1 \mathrm{H}), 3.02-2.74(\mathrm{~m}, 2 \mathrm{H}), 1.70-1.46(\mathrm{~m}, 2 \mathrm{H}), 0.93$ $(\mathrm{t}, J=7.4 \mathrm{~Hz}, 3 \mathrm{H}) ;{ }^{13} \mathrm{C} \mathrm{NMR}\left(125 \mathrm{MHz}, \mathrm{CDCl}_{3}\right): \delta 201.98,138.22$, 128.84, 128.74, 127.08, 79.95, 30.84, 22.64, 13.29.

HPLC analysis: Chiralcel OD-H, Hex/IPA = 95/5, $1.0 \mathrm{~mL} / \mathrm{min}, 220 \mathrm{~nm}$; $\mathrm{t}_{R}=8.8 \min (\operatorname{minr}, S), 13.6 \min ($ major,$R)$

The analytical data was identical to the reported value. ${ }^{4}$

Compound 4ac: Isolated yield: $19.5 \mathrm{mg}(93 \%)$; colorless liquid<smiles>CC(C)SC(=O)[C@H](O)c1ccccc1</smiles>

$(R)-4$ ac

${ }^{1} \mathrm{H}$ NMR $\left(500 \mathrm{MHz}, \mathrm{CDCl}_{3}\right): \delta 7.44-7.34(\mathrm{~m}, 5 \mathrm{H}), 5.18(\mathrm{~d}, J=4.4 \mathrm{~Hz}$, $1 \mathrm{H}), 3.72-3.63(\mathrm{~m}, 1 \mathrm{H}), 3.60(\mathrm{t}, J=4.2 \mathrm{~Hz}, 1 \mathrm{H}), 1.28(\mathrm{dd}, J=16.5,6.9 \mathrm{~Hz}$, $6 \mathrm{H}) ;{ }^{13} \mathrm{C}$ NMR $\left(125 \mathrm{MHz}, \mathrm{CDCl}_{3}\right): \delta 201.79,138.27,128.86,128.78$, 127.18, 79.96, 35.22, 22.90, 22.84.

HPLC analysis: Chiralcel OD-H, Hex/IPA = 95/5, $1.0 \mathrm{~mL} / \mathrm{min}, 220 \mathrm{~nm} ; \mathrm{t}_{R}$ $=8.1 \min (\operatorname{minor}, S), 14.9 \min ($ major,$R)$

The analytical data was identical to the reported value. ${ }^{4}$

Compound 4ad: Isolated yield: $20.3 \mathrm{mg}(90 \%)$; colorless liquid

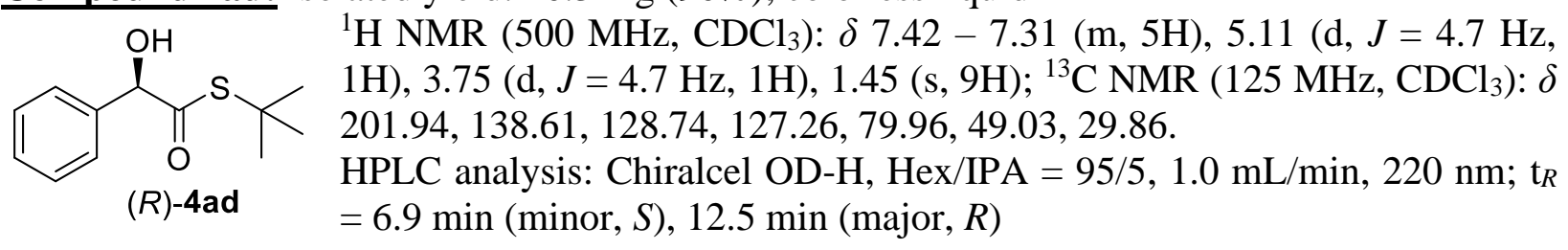

The analytical data was identical to the reported value. ${ }^{4}$ 
Compound 4ae: Isolated yield: $23.7 \mathrm{mg}$ (97\%); colorless liquid<smiles>O=C(Sc1ccccc1)C(O)c1ccccc1</smiles>

$(R)-4 a e$

${ }^{1} \mathrm{H}$ NMR $\left(500 \mathrm{MHz}, \mathrm{CDCl}_{3}\right): \delta 7.60-7.32(\mathrm{~m}, 10 \mathrm{H}), 5.34(\mathrm{~d}, J=4.3 \mathrm{~Hz}$, $1 \mathrm{H}), 3.47(\mathrm{~d}, J=4.5 \mathrm{~Hz}, 1 \mathrm{H}) ;{ }^{13} \mathrm{C} \mathrm{NMR}\left(125 \mathrm{MHz}, \mathrm{CDCl}_{3}\right): \delta 200.13$, 137.72, 134.64, 129.67, 129.31, 129.10, 128.92, 127.21, 126.54, 80.03. HPLC analysis: Chiralcel OD-H, Hex/IPA = 95/5, $1.0 \mathrm{~mL} / \mathrm{min}, 220 \mathrm{~nm}$; $\mathrm{t}_{R}=18.5 \mathrm{~min}$ (minor, $S$ ), 22.2 min (major, $R$ )

The analytical data was identical to the reported value. ${ }^{4}$

Compound 4af: Isolated yield: $28.1 \mathrm{mg}(96 \%)$; white solid<smiles>O=C(Sc1cccc2ccccc12)C(O)c1ccccc1</smiles>

(R)-4af

${ }^{1} \mathrm{H}$ NMR $\left(500 \mathrm{MHz}, \mathrm{CDCl}_{3}\right): \delta 7.97-7.80(\mathrm{~m}, 3 \mathrm{H}), 7.71-7.58(\mathrm{~m}$, $1 \mathrm{H}), 7.55-7.36(\mathrm{~m}, 8 \mathrm{H}), 5.38(\mathrm{~s}, 1 \mathrm{H}), 3.60(\mathrm{~s}, 1 \mathrm{H}) ;{ }^{13} \mathrm{C}$ NMR $(125$ $\left.\mathrm{MHz}, \mathrm{CDCl}_{3}\right): \delta 199.90,137.85,135.28,134.25,134.21,131.22$, $129.15,128.99,128.74,127.32,127.24,126.53,125.63,125.00$, 123.93, 80.17.

HPLC analysis: Chiralcel OD-H, Hex/IPA = 90/10, $1.0 \mathrm{~mL} / \mathrm{min}, 220$ $\mathrm{nm} ; \mathrm{t}_{R}=18.0 \min ($ major, $R), 27.5 \min (\operatorname{minor}, S)$

The analytical data was identical to the reported value. ${ }^{4}$

Compound 4ag: Isolated yield: $3 \mathrm{mg}$ (12\%); colorless liquid<smiles>O=C(Sc1cccs1)[C@@H](O)c1ccccc1</smiles>

${ }^{1} \mathrm{H} \mathrm{NMR}\left(500 \mathrm{MHz}, \mathrm{CDCl}_{3}\right): \delta 7.57-7.51(\mathrm{~m}, 1 \mathrm{H}), 7.47-7.44(\mathrm{~m}, 2 \mathrm{H})$, $7.42-7.38(\mathrm{~m}, 3 \mathrm{H}), 7.13-7.04(\mathrm{~m}, 2 \mathrm{H}), 5.33(\mathrm{~s}, 1 \mathrm{H}), 3.44(\mathrm{~s}, 1 \mathrm{H}) ;{ }^{13} \mathrm{C}$ NMR (125 MHz, $\left.\mathrm{CDCl}_{3}\right): \delta$ 200.08, 137.24, 136.09, 132.11, 129.22, 128.99, 127.92, 127.15, 123.35, 79.92.

HPLC analysis: Chiralpak IC, Hex/IPA = 95/5, $1.0 \mathrm{~mL} / \mathrm{min}, 220 \mathrm{~nm} ; \mathrm{t}_{R}=$ $11.8 \min$ (major, $R$ ), 14.4 min (minor, $S$ )

The analytical data was identical to the reported value. ${ }^{4}$

Compound 4bd: New compound, isolated yield: $22.6 \mathrm{mg}$ (82\%); white solid; mp: $123{ }^{\circ} \mathrm{C}$; TLC<smiles>CC(C)(C)SC(=O)[C@@H](O)c1ccc2ccccc2c1</smiles>
$($ EtOAc/ $n$-hexane, $1 / 4 \mathrm{v} / \mathrm{v}): \mathrm{R}_{f}=0.51 ;[\alpha]_{\mathrm{D}}^{20}=-209\left(\mathrm{c}=0.8\right.$ in $\left.\mathrm{CHCl}_{3}\right)$; ${ }^{1} \mathrm{H}$ NMR $\left(500 \mathrm{MHz}, \mathrm{CDCl}_{3}\right): \delta 7.93-7.74(\mathrm{~m}, 4 \mathrm{H}), 7.56-7.38(\mathrm{~m}$, $3 \mathrm{H}), 5.27(\mathrm{~d}, J=4.6 \mathrm{~Hz}, 1 \mathrm{H}), 3.88(\mathrm{~d}, J=4.6 \mathrm{~Hz}, 1 \mathrm{H}), 1.44(\mathrm{~s}, 9 \mathrm{H})$; ${ }^{13} \mathrm{C}$ NMR (125 MHz, $\left.\mathrm{CDCl}_{3}\right): \delta$ 201.90, 135.98, 133.46, 133.24, $(R)-\mathbf{4 b d}$ $128.69,128.20,127.79,127.07,126.44,126.35,124.33,80.13,49.11$,

29.87.

IR (neat): $v$ 1669, 1507, 1456, 1363, 1155, 973, 955, 810.

HRMS (m/z, ESI): [M+Na] ${ }^{+}$cald. for $\mathrm{C}_{16} \mathrm{H}_{18} \mathrm{OS}_{2} \mathrm{Na}, 297.0925$; found, 297.0922.

HPLC analysis: Chiralpak IA, Hex/IPA $=95 / 5,1.0 \mathrm{~mL} / \mathrm{min}, 220 \mathrm{~nm}$; $\mathrm{t}_{R}=13.2 \mathrm{~min}$ (minor, $S$ ), 15.0 min (major, $R$ ) 
Compound 4cd: New compound, isolated yield: $14.3 \mathrm{mg}(60 \%)$; white solid; mp: $73{ }^{\circ} \mathrm{C}$; TLC<smiles>Cc1ccc(C(O)C(=O)SC(C)(C)C)cc1</smiles>

$(R)-4 \mathrm{~cd}$

$($ EtOAc/ $n$-hexane, $1 / 4 \mathrm{v} / \mathrm{v}): \mathrm{R}_{f}=0.48 ;[\alpha]_{\mathrm{D}}^{20}=-142\left(\mathrm{c}=0.5\right.$ in $\left.\mathrm{CHCl}_{3}\right)$;

${ }^{1} \mathrm{H}$ NMR $\left(500 \mathrm{MHz}, \mathrm{CDCl}_{3}\right): \delta 7.26(\mathrm{~d}, J=8.1 \mathrm{~Hz}, 2 \mathrm{H}), 7.18(\mathrm{~d}, J=7.9$ $\mathrm{Hz}, 2 \mathrm{H}), 5.06(\mathrm{~d}, J=4.7 \mathrm{~Hz}, 1 \mathrm{H}), 3.67(\mathrm{~d}, J=4.8 \mathrm{~Hz}, 1 \mathrm{H}), 2.35$ (s, 3H), $1.45(\mathrm{~s}, 9 \mathrm{H}) ;{ }^{13} \mathrm{C} \mathrm{NMR}\left(125 \mathrm{MHz}, \mathrm{CDCl}_{3}\right): \delta 202.11,138.58,135.70$, $129.45,127.20,79.82,48.92,29.87,21.28$.

IR (neat): $v 1677,1609,1479,1455,1365,1260,1156,1051,969,902,779$.

HRMS (m/z, ESI): [M+Na] $]^{+}$cald. for $\mathrm{C}_{16} \mathrm{H}_{18} \mathrm{OS}_{2} \mathrm{Na}, 261.0925$; found, 261.0922 .

HPLC analysis: Chiralpak IA, Hex/IPA = 95/5, $1.0 \mathrm{~mL} / \mathrm{min}, 220 \mathrm{~nm} ; \mathrm{t}_{R}=9.1 \mathrm{~min}(\operatorname{minor}, S$ ), 11.6 min (major, $R$ )

Compound 4dd: New compound, isolated yield: $15.5 \mathrm{mg}$ (65\%); colorless liquid; TLC<smiles>Cc1cccc(C(O)C(=O)SC(C)(C)C)c1</smiles>

$(R)-4 \mathrm{dd}$

$($ EtOAc/ $n$-hexane, $1 / 4 \mathrm{v} / \mathrm{v}): \mathrm{R}_{f}=0.47 ;[\alpha]_{\mathrm{D}}{ }^{20}=-166\left(\mathrm{c}=0.5\right.$ in $\left.\mathrm{CHCl}_{3}\right)$;

${ }^{1} \mathrm{H}$ NMR $\left(500 \mathrm{MHz}, \mathrm{CDCl}_{3}\right): \delta 7.28-7.24(\mathrm{~m}, 1 \mathrm{H}), 7.20-7.13(\mathrm{~m}, 3 \mathrm{H})$, $5.06(\mathrm{~d}, J=4.5 \mathrm{~Hz}, 1 \mathrm{H}), 3.70(\mathrm{~d}, J=4.7 \mathrm{~Hz}, 1 \mathrm{H}), 2.36(\mathrm{~s}, 3 \mathrm{H}), 1.45(\mathrm{~s}$, $9 \mathrm{H}) ;{ }^{13} \mathrm{C}$ NMR $\left(125 \mathrm{MHz}, \mathrm{CDCl}_{3}\right): \delta 202.02,138.53,138.46,129.50$, $128.60,127.87,124.39,80.00,48.94,29.88,21.44$.

IR (neat): v1677, 1607, 1477, 1455, 1365, 1155, 1078, 1054, 969, 901, 776, 760, 718.

HRMS (m/z, ESI): [M+Na] $]^{+}$cald. for $\mathrm{C}_{16} \mathrm{H}_{18} \mathrm{OS}_{2} \mathrm{Na}, 261.0925$; found, 261.0923 .

HPLC analysis: Chiralpak IA, Hex/IPA =99/1, $1.0 \mathrm{~mL} / \mathrm{min}, 220 \mathrm{~nm} ; \mathrm{t}_{R}=16.8 \mathrm{~min}(\operatorname{minor}, S)$, 18.9 min (major, $R$ )

Compound 4ed: New compound, isolated yield: $10.1 \mathrm{mg}$ (40\%); colorless liquid; TLC<smiles>COc1ccc([C@@H](O)C(=O)SC(C)(C)C)cc1</smiles>
$($ EtOAc/ $n$-hexane, $1 / 4 \mathrm{v} / \mathrm{v}): \mathrm{R}_{f}=0.50 ;[\alpha]_{\mathrm{D}}{ }^{20}=-94\left(\mathrm{c}=1.0\right.$ in $\left.\mathrm{CHCl}_{3}\right)$; ${ }^{1} \mathrm{H}$ NMR $\left(500 \mathrm{MHz}, \mathrm{CDCl}_{3}\right): \delta 7.30-7.29(\mathrm{~m}, 2 \mathrm{H}), 6.92-6.89(\mathrm{~m}$, $2 \mathrm{H}), 5.05(\mathrm{~d}, J=4.6 \mathrm{~Hz}, 1 \mathrm{H}), 3.81(\mathrm{~s}, 3 \mathrm{H}), 3.64(\mathrm{~d}, J=4.6 \mathrm{~Hz}, 1 \mathrm{H})$, $1.45(\mathrm{~s}, 9 \mathrm{H}) ;{ }^{13} \mathrm{C} \mathrm{NMR}\left(125 \mathrm{MHz}, \mathrm{CDCl}_{3}\right): \delta 202.28,159.93,130.78$, $128.62,114.15,79.52,55.27,48.83,29.88$.

IR (neat): $v 1675,1600,1489,1455,1365,1260,1157,1045,972,901,830$.

HRMS (m/z, ESI): [M+Na $]^{+}$cald. for $\mathrm{C}_{13} \mathrm{H}_{18} \mathrm{O}_{3} \mathrm{SNa}, 277.0874$; found, 277.0871 .

HPLC analysis: Chiralpak IA, Hex/IPA = 95/5, $1.0 \mathrm{~mL} / \mathrm{min}, 220 \mathrm{~nm}$; $\mathrm{t}_{R}=13.2 \mathrm{~min}(\operatorname{minor}, S$ ), 16.4 min (major, $R$ )

Compound 4fd: New compound, isolated yield: $17.7 \mathrm{mg}(73 \%)$; white solid; mp: $40{ }^{\circ} \mathrm{C}$; TLC<smiles>CC(C)(C)SC(=O)[C@@H](O)c1ccc(F)cc1</smiles>
$($ EtOAc/ $n$-hexane, $1 / 4 \mathrm{v} / \mathrm{v}): \mathrm{R}_{f}=0.52 ;[\alpha]_{\mathrm{D}}{ }^{20}=-62.5\left(\mathrm{c}=1.2\right.$ in $\left.\mathrm{CHCl}_{3}\right)$; ${ }^{1} \mathrm{H}$ NMR $\left(500 \mathrm{MHz}, \mathrm{CDCl}_{3}\right): \delta 7.38-7.35(\mathrm{~m}, 2 \mathrm{H}), 7.08-7.04(\mathrm{~m}, 2 \mathrm{H})$, $5.09(\mathrm{~d}, J=4.49 \mathrm{~Hz}, 1 \mathrm{H}), 3.74(\mathrm{~d}, J=4.51 \mathrm{~Hz}, 1 \mathrm{H}), 1.45(\mathrm{~s}, 9 \mathrm{H}) ;{ }^{13} \mathrm{C}$ NMR $\left(125 \mathrm{MHz}, \mathrm{CDCl}_{3}\right): \delta 201.81,162.93\left(\mathrm{~d},{ }^{1} J_{\mathrm{C}-\mathrm{F}}=247.39 \mathrm{~Hz}\right)$, $134.44\left(\mathrm{~d},{ }^{4} J_{\mathrm{C}-\mathrm{F}}=3.17 \mathrm{~Hz}\right), 129.01\left(\mathrm{~d},{ }^{3} J_{\mathrm{C}-\mathrm{F}}=8.40 \mathrm{~Hz}\right), 115.68\left(\mathrm{~d},{ }^{2} J_{\mathrm{C}-\mathrm{F}}\right.$ $=21.72 \mathrm{~Hz}), 79.21,49.10,29.84 ;{ }^{19} \mathrm{~F} \mathrm{NMR}\left(470 \mathrm{MHz}, \mathrm{CDCl}_{3}\right): \delta-113.04$.

IR (neat): $v$ 2364, 2338, 1667, 1602, 1509, 1364, 1233, 1186, 1156, 1104, 1078, 1014, 971, 937, $851,808$.

HRMS (m/z, ESI): [M+Na] ${ }^{+}$cald. for $\mathrm{C}_{12} \mathrm{H}_{15} \mathrm{FO}_{2} \mathrm{SNa}$, 265.0675; found, 265.0672. 
HPLC analysis: Chiralpak IA, Hex/IPA = 95/5, $1.0 \mathrm{~mL} / \mathrm{min}, 220 \mathrm{~nm} ; \mathrm{t}_{R}=7.8 \mathrm{~min}(\operatorname{minor}, S), 9.1$ $\min ($ major, $R$ )

Compound 4gd: New compound, isolated yield: $27.5 \mathrm{mg}$ (95\%); colorless liquid; TLC<smiles>CC(C)(C)SC(=O)[C@@H](O)c1ccc(F)cc1F</smiles>
$($ EtOAc/ $n$-hexane, $1 / 4 \mathrm{v} / \mathrm{v}): \mathrm{R}_{f}=0.63 ;[\alpha]_{\mathrm{D}}{ }^{20}=-75\left(\mathrm{c}=1.0\right.$ in $\left.\mathrm{CHCl}_{3}\right)$;

${ }^{1} \mathrm{H}$ NMR $\left(500 \mathrm{MHz}, \mathrm{CDCl}_{3}\right): \delta 7.35-7.30(\mathrm{~m}, 1 \mathrm{H}), 6.92-6.82(\mathrm{~m}, 2 \mathrm{H})$, $5.32(\mathrm{~d}, J=4.70 \mathrm{~Hz}, 1 \mathrm{H}), 3.82(\mathrm{~d}, J=4.70 \mathrm{~Hz}, 1 \mathrm{H}), 1.47(\mathrm{~s}, 9 \mathrm{H}) ;{ }^{13} \mathrm{C}$ NMR $\left(125 \mathrm{MHz}, \mathrm{CDCl}_{3}\right): \delta 200.90,163.18,\left(\mathrm{dd},{ }^{1,3} J_{\mathrm{C}-\mathrm{F}}=12.06,250.42\right.$ $\mathrm{Hz}), 160.82\left(\mathrm{dd},{ }^{1,3} J_{\mathrm{C}-\mathrm{F}}=12.15,251.26 \mathrm{~Hz}\right), 130.07\left(\mathrm{dd}^{3,3} J_{\mathrm{C}-\mathrm{F}}=5.11\right.$, $9.96 \mathrm{~Hz}), 122.34\left(\mathrm{dd},{ }^{2,4} J_{\mathrm{C}-\mathrm{F}}=3.81,13.61 \mathrm{~Hz}\right), 111.81\left(\mathrm{dd},{ }^{2,4} J_{\mathrm{C}-\mathrm{F}}=3.68,21.45 \mathrm{~Hz}\right), 104.29(\mathrm{t}$, $\left.{ }^{2,2} J_{\mathrm{C}-\mathrm{F}}=25.47,25.47 \mathrm{~Hz}\right), 73.58\left(\mathrm{~d},{ }^{4} J_{\mathrm{C}-\mathrm{F}}=2.26 \mathrm{~Hz}\right), 49.27,29.82 ;{ }^{19} \mathrm{~F} \mathrm{NMR}\left(470 \mathrm{MHz}, \mathrm{CDCl}_{3}\right)$ : $\delta-108.78(\mathrm{~d}, J=8.2 \mathrm{~Hz}),-113.40(\mathrm{~d}, J=8.11 \mathrm{~Hz})$.

IR (neat): v 2364, 2338, 1667, 1602, 1509, 1364, 1233, 1186, 1156, 1104, 1078, 1014, 971, 937 , 851,808 .

HRMS (m/z, ESI): [M+Na] ${ }^{+}$cald. for $\mathrm{C}_{12} \mathrm{H}_{14} \mathrm{~F}_{2} \mathrm{O}_{2} \mathrm{SNa}$, 283.0580; found, 283.0579.

HPLC analysis: Chiralpak IA, Hex/IPA = 95/5, $1.0 \mathrm{~mL} / \mathrm{min}, 220 \mathrm{~nm} ; \mathrm{t}_{R}=8.4 \mathrm{~min}(\operatorname{minor}, S$ ), 9.9 $\min ($ major, $R$ )

Compound 4hd: New compound, isolated yield: $18.1 \mathrm{mg}$ (70\%); colorless liquid; TLC<smiles>CC(C)(C)SC(=O)[C@@H](O)c1ccc(Cl)cc1</smiles>
$($ EtOAc/ $n$-hexane, $1 / 4 \mathrm{v} / \mathrm{v}): \mathrm{R}_{f}=0.51 ;[\alpha]_{\mathrm{D}}{ }^{20}=-160\left(\mathrm{c}=0.63\right.$ in $\left.\mathrm{CHCl}_{3}\right)$; ${ }^{1} \mathrm{H}$ NMR $\left(500 \mathrm{MHz}, \mathrm{CDCl}_{3}\right): \delta 7.38-7.30(\mathrm{~m}, 4 \mathrm{H}), 5.08(\mathrm{~s}, 1 \mathrm{H}), 3.77$ $(\mathrm{s}, 1 \mathrm{H}), 1.45(\mathrm{~s}, 9 \mathrm{H}) ;{ }^{13} \mathrm{C} \mathrm{NMR}\left(125 \mathrm{MHz}, \mathrm{CDCl}_{3}\right): \delta 201.45,137.08$, 134.61, 128.91, 128.56, 79.23, 49.29, 29.84.

IR (neat): $v$ 1672, 1491, 1455, 1407, 1392, 1365, 1189, 1167, 1089, $1055,1015,969,847,802,744$.

HRMS (m/z, ESI): [M+Na] ${ }^{+}$cald. for $\mathrm{C}_{12} \mathrm{H}_{15} \mathrm{ClO}_{2} \mathrm{SNa}$, 281.0379; found, 281.0375.

HPLC analysis: Chiralpak IA, Hex/IPA = 99/1, $1.0 \mathrm{~mL} / \mathrm{min}, 220 \mathrm{~nm}$; $\mathrm{t}_{R}=19.8 \mathrm{~min}(\operatorname{minor}, S$ ), 23.2 min (major, $R$ )

Compound 4id: New compound, isolated yield: $23.1 \mathrm{mg}$ (95\%); colorless liquid; TLC<smiles>CC(C)(C)SC(=O)C(O)c1cccc(F)c1</smiles>

$(R)-4$ id $($ EtOAc/ $n$-hexane, $1 / 4 \mathrm{v} / \mathrm{v}): \mathrm{R}_{f}=0.42 ;[\alpha]_{\mathrm{D}}{ }^{20}=-52\left(\mathrm{c}=0.25\right.$ in $\left.\mathrm{CHCl}_{3}\right)$; ${ }^{1} \mathrm{H}$ NMR (500 MHz, $\left.\mathrm{CDCl}_{3}\right): \delta 7.38-7.30(\mathrm{~m}, 1 \mathrm{H}), 7.21-7.16(\mathrm{~m}, 1 \mathrm{H})$, $7.13-7.09(\mathrm{~m}, 1 \mathrm{H}), 7.08-6.99(\mathrm{~m}, 1 \mathrm{H}), 5.10(\mathrm{~d}, J=4.6 \mathrm{~Hz}, 1 \mathrm{H}), 3.80$ $(\mathrm{d}, J=4.7 \mathrm{~Hz}, 1 \mathrm{H}), 1.45(\mathrm{~s}, 9 \mathrm{H}) . ;{ }^{13} \mathrm{C} \mathrm{NMR}\left(125 \mathrm{MHz}, \mathrm{CDCl}_{3}\right): \delta$ 201.28, $162.90\left(\mathrm{~d},{ }^{1} J_{\mathrm{C}-\mathrm{F}}=246.8 \mathrm{~Hz}\right), 140.98\left(\mathrm{~d},{ }^{3} J_{\mathrm{C}-\mathrm{F}}=7.1 \mathrm{~Hz}\right), 130.20$ $\left(\mathrm{d},{ }^{3} J_{\mathrm{C}-\mathrm{F}}=8.2 \mathrm{~Hz}\right), 122.91\left(\mathrm{~d},{ }^{4} J_{\mathrm{C}-\mathrm{F}}=2.9 \mathrm{~Hz}\right), 115.64\left(\mathrm{~d},{ }^{2} J_{\mathrm{C}-\mathrm{F}}=21.2 \mathrm{~Hz}\right), 114.07\left(\mathrm{~d},{ }^{2} J_{\mathrm{C}-\mathrm{F}}=22.4\right.$ $\mathrm{Hz}), 79.27\left(\mathrm{~d},{ }^{4} J_{\mathrm{C}-\mathrm{F}}=1.8 \mathrm{~Hz}\right), 49.27,29.83$.

IR (neat): $v$ 1674, 1592, 1486, 1452, 1366, 1249, 1162, 1137, 1064, 975, 921, 756.

HRMS (m/z, ESI): [M+Na] ${ }^{+}$cald. for $\mathrm{C}_{12} \mathrm{H}_{15} \mathrm{FO}_{2} \mathrm{SNa}, 265.0675$; found, 265.0673.

HPLC analysis: Chiralcel OX-3, Hex/IPA = 99/1, $1.0 \mathrm{~mL} / \mathrm{min}, 220 \mathrm{~nm}$; $\mathrm{t}_{R}=12.6 \mathrm{~min}$ (minor, $S$ ), 15.1 min (major, $R$ ) 
Compound 4jd: New compound, isolated yield: $24.8 \mathrm{mg}$ (96\%); colorless liquid; TLC<smiles>CC(C)(C)SC(=O)C(O)c1cccc(Cl)c1</smiles>

$(R)-4 \mathbf{j d}$ $($ EtOAc/ $n$-hexane, $1 / 4 \mathrm{v} / \mathrm{v}): \mathrm{R}_{f}=0.42 ;[\alpha]_{\mathrm{D}}{ }^{20}=-122\left(\mathrm{c}=0.5\right.$ in $\left.\mathrm{CHCl}_{3}\right)$; ${ }^{1} \mathrm{H}$ NMR $\left(500 \mathrm{MHz}, \mathrm{CDCl}_{3}\right): \delta 7.40-7.38(\mathrm{~m}, 1 \mathrm{H}), 7.33-7.27(\mathrm{~m}$, $3 \mathrm{H}), 5.07(\mathrm{~d}, J=4.7 \mathrm{~Hz}, 1 \mathrm{H}), 3.80(\mathrm{~d}, J=4.7 \mathrm{~Hz}, 1 \mathrm{H}), 1.46(\mathrm{~s}, 9 \mathrm{H}) ;{ }^{13} \mathrm{C}$ NMR (125 MHz, $\left.\mathrm{CDCl}_{3}\right): \delta$ 201.22, 140.51, 134.61, 129.92, 128.85, $127.29,125.38,79.25,49.36,29.85$.

IR (neat): $v$ 1672, 1595, 1576, 1475, 1457, 1431, 1365, 1192, 1164, 1076, 969, 779, 744, 729, 685,630 .

HRMS (m/z, ESI): [M+Na] ${ }^{+}$cald. for $\mathrm{C}_{12} \mathrm{H}_{15} \mathrm{ClO}_{2} \mathrm{SNa}$, 281.0379; found, 281.0976.

HPLC analysis: Chiralcel OX-3, Hex/IPA = 99/1, $1.0 \mathrm{~mL} / \mathrm{min}, 220 \mathrm{~nm}$; $\mathrm{t}_{R}=14.1 \mathrm{~min}(\operatorname{minor}, S)$, 15.0 min (major, $R$ )

Compound 4kd: New compound, isolated yield: $28.4 \mathrm{mg}$ (94\%); colorless liquid; TLC<smiles>CC(C)(C)SC(=O)C(O)c1cccc(Br)c1</smiles>

$(R)-\mathbf{4 k d}$

$($ EtOAc/ $n$-hexane, $1 / 4 \mathrm{v} / \mathrm{v}): \mathrm{R}_{f}=0.55 ;[\alpha]_{\mathrm{D}}^{20}=-105\left(\mathrm{c}=0.95\right.$ in $\left.\mathrm{CHCl}_{3}\right)$; ${ }^{1} \mathrm{H}$ NMR $\left(500 \mathrm{MHz}, \mathrm{CDCl}_{3}\right): \delta 7.57-7.54(\mathrm{~m}, 1 \mathrm{H}), 7.49-7.45(\mathrm{~m}$, $1 \mathrm{H}), 7.34-7.31(\mathrm{~m}, 1 \mathrm{H}), 7.26-7.22(\mathrm{~m}, 1 \mathrm{H}), 5.06(\mathrm{~d}, J=4.5 \mathrm{~Hz}, 1 \mathrm{H})$, $3.79(\mathrm{~d}, J=4.7 \mathrm{~Hz}, 1 \mathrm{H}), 1.46(\mathrm{~s}, 9 \mathrm{H}) ;{ }^{13} \mathrm{C} \mathrm{NMR}\left(125 \mathrm{MHz}, \mathrm{CDCl}_{3}\right): \delta$ 201.18, 140.74, 131.76, 130.19, 130.17, 125.84, 122.74, 79.18, 49.37,

29.84 .

IR (neat): $v$ 2360, 1671, 1593, 1571, 1474, 1456, 1427, 1365, 1186, 1166, 1098, 1071, 1055, 968. HRMS (m/z, ESI): [M+Na] ${ }^{+}$cald. for $\mathrm{C}_{12} \mathrm{H}_{15} \mathrm{BrO}_{2} \mathrm{SNa}$, 324.9874; found, 324.9870.

HPLC analysis: Chiralcel OX-3, Hex/IPA = 99/1, $1.0 \mathrm{~mL} / \mathrm{min}, 220 \mathrm{~nm}$; $\mathrm{t}_{R}=14.6 \mathrm{~min}$ (minor, $S$ ), 16.2 min (major, $R$ )

Compound 4ld: New compound, isolated yield: $22.1 \mathrm{mg}$ (97\%); colorless liquid; TLC

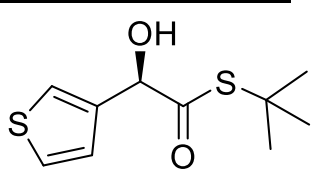

$(R)-4 \mid d$ $($ EtOAc/ $n$-hexane, $1 / 4 \mathrm{v} / \mathrm{v}): \mathrm{R}_{f}=0.57 ;[\alpha]_{\mathrm{D}}{ }^{20}=-61\left(\mathrm{c}=1.0\right.$ in $\left.\mathrm{CHCl}_{3}\right)$; ${ }^{1} \mathrm{H}$ NMR $\left(500 \mathrm{MHz}, \mathrm{CDCl}_{3}\right): \delta 7.36-7.34(\mathrm{~m}, 1 \mathrm{H}), 7.34-7.31(\mathrm{~m}, 1 \mathrm{H})$, $7.09(\mathrm{dd}, J=5.0,1.1 \mathrm{~Hz}, 1 \mathrm{H}), 5.21(\mathrm{~d}, J=5.0 \mathrm{~Hz}, 1 \mathrm{H}), 3.57(\mathrm{~d}, J=5.2 \mathrm{~Hz}$, $1 \mathrm{H}), 1.47(\mathrm{~s}, 9 \mathrm{H}) ;{ }^{13} \mathrm{C}$ NMR $\left(125 \mathrm{MHz}, \mathrm{CDCl}_{3}\right): \delta 201.38,139.32,126.49$, 125.94, 123.77, 75.96, 48.92, 29.85.

IR (neat): v 1676, 1476, 11455, 1364, 1154, 1082, 1050, 975, 927, 913, 837, 778, 744.

HRMS (m/z, ESI): [M+Na] $]^{+}$cald. for $\mathrm{C}_{10} \mathrm{H}_{14} \mathrm{O}_{2} \mathrm{SNa}$, 253.0333; found, 253.0331 .

HPLC analysis: Chiralpak IA, Hex/IPA $=95 / 5,1.0 \mathrm{~mL} / \mathrm{min}, 220 \mathrm{~nm} ; \mathrm{t}_{R}=11.2 \mathrm{~min}(\operatorname{minor}, S$ ), 12.7 min (major, $R$ )

Compound 4md: New compound, isolated yield: $3.9 \mathrm{mg}(24 \%) ;{ }^{1} \mathrm{H}$ NMRconversion: 80\%;<smiles>CC(O)C(=O)SC(C)(C)C</smiles>

$(R)-4 \mathrm{md}$ colorless liquid; TLC (EtOAc/ $n$-hexane, $1 / 4 \mathrm{v} / \mathrm{v}): \mathrm{R}_{f}=0.43 ;[\alpha]_{\mathrm{D}}{ }^{20}=+60(\mathrm{c}=$ 0.13 in $\left.\mathrm{CHCl}_{3}\right)$;

${ }^{1} \mathrm{H}$ NMR $\left(500 \mathrm{MHz}, \mathrm{CDCl}_{3}\right): \delta 4.24(\mathrm{q}, J=6.8 \mathrm{~Hz}, 1 \mathrm{H}), 2.94(\mathrm{~s}, 1 \mathrm{H}), 1.50(\mathrm{~s}$, 9H), $1.42(\mathrm{~d}, J=6.8 \mathrm{~Hz}, 3 \mathrm{H}) ;{ }^{13} \mathrm{C} \mathrm{NMR}\left(125 \mathrm{MHz}, \mathrm{CDCl}_{3}\right): \delta$ 204.46, 73.84, 48.30, 29.91, 21.52.

IR (neat): $v 1668,1455,1365,1148,1139,1037,964,915,887,738,666,652,638,626$.

HRMS (m/z, ESI): [M+Na] ${ }^{+}$cald. for $\mathrm{C}_{7} \mathrm{H}_{14} \mathrm{O}_{2} \mathrm{SNa}$, 185.0612; found, 185.0610 . 
HPLC analysis: Chiralcel OX-3, Hex/IPA = 99/1, $0.7 \mathrm{~mL} / \mathrm{min}, 220 \mathrm{~nm} ; \mathrm{t}_{R}=12.4 \mathrm{~min}(\operatorname{minor}, S$ ), $12.9 \min ($ major, $R$ )

Compound 4nd: New compound, isolated yield: $15.6 \mathrm{mg}(83 \%)$; colorless liquid; TLC<smiles>CCC[C@H](O)C(=O)SC(C)(C)C</smiles>
$($ EtOAc/ $n$-hexane, $1 / 4 \mathrm{v} / \mathrm{v}): \mathrm{R}_{f}=0.52 ;[\alpha]_{\mathrm{D}}^{20}=-33\left(\mathrm{c}=0.75\right.$ in $\left.\mathrm{CHCl}_{3}\right)$; (R)-4nd ${ }^{1} \mathrm{H}$ NMR $\left(500 \mathrm{MHz}, \mathrm{CDCl}_{3}\right): \delta 4.18-4.12(\mathrm{~m}, 1 \mathrm{H}), 2.92(\mathrm{~d}, J=5.4 \mathrm{~Hz}$, $1 \mathrm{H}), 1.83-1.72(\mathrm{~m}, 1 \mathrm{H}), 1.65-1.55(\mathrm{~m}, 1 \mathrm{H}), 1.50-1.40(\mathrm{~m}, 11 \mathrm{H}), 0.95(\mathrm{t}$, $J=7.4 \mathrm{~Hz}, 3 \mathrm{H}) ;{ }^{13} \mathrm{C} \mathrm{NMR}\left(125 \mathrm{MHz}, \mathrm{CDCl}_{3}\right): \delta 204.18,77.33,48.30$, $37.69,29.92,17.86,13.84$.

IR (neat): v 1676, 1477, 1456, 1391, 1364, 1163, 1141, 1068, 1033, 993, 940, 931, 740, 732.

HRMS (m/z, ESI): [M+Na] ${ }^{+}$cald. for $\mathrm{C}_{9} \mathrm{H}_{18} \mathrm{O}_{2} \mathrm{SNa}, 213.0925$; found, 213.0923.

HPLC analysis: Chiralpak IA-3, Hex/IPA = 99/1, $1.0 \mathrm{~mL} / \mathrm{min}, 220 \mathrm{~nm} ; \mathrm{t}_{R}=12.0 \mathrm{~min}(\operatorname{minor}, S)$, $12.9 \min ($ major, $R$ )

Compound 4od: New compound, isolated yield: $18.3 \mathrm{mg}(24 \%)$; colorless liquid; TLC<smiles>CCCCCC(O)C(=O)SC(C)(C)C</smiles>

(R)-4od $($ EtOAc/ $n$-hexane, $1 / 4 \mathrm{v} / \mathrm{v}): \mathrm{R}_{f}=0.55 ;[\alpha]_{\mathrm{D}}{ }^{20}=-105\left(\mathrm{c}=0.8\right.$ in $\left.\mathrm{CHCl}_{3}\right)$; ${ }^{1} \mathrm{H} \mathrm{NMR}\left(500 \mathrm{MHz}, \mathrm{CDCl}_{3}\right): \delta 4.17-4.12(\mathrm{~m}, 1 \mathrm{H}), 2.88(\mathrm{~d}, J=5.4$ $\mathrm{Hz}, 1 \mathrm{H}), 1.85-1.76(\mathrm{~m}, 1 \mathrm{H}), 1.66-1.57(\mathrm{~m}, 2 \mathrm{H}), 1.50(\mathrm{~s}, 9 \mathrm{H}), 1.48-$ $1.39(\mathrm{~m}, 3 \mathrm{H}), 1.32-1.29(\mathrm{~m}, 2 \mathrm{H}), 0.89(\mathrm{t}, J=6.8 \mathrm{~Hz}, 3 \mathrm{H}) ;{ }^{13} \mathrm{C}$ NMR $\left(125 \mathrm{MHz} \mathrm{CDCl}_{3}\right): \delta 204.15,77.51,48.33,35.58,31.53,29.93,24.13$,

$22.46,13.97$.

IR (neat): $v$ 1676, 1477, 1456, 1391, 1379, 1365, 1163, 1139, 1079, 1058, 965, 910, 732.

HRMS (m/z, ESI): [M+Na] $]^{+}$cald. for $\mathrm{C}_{11} \mathrm{H}_{22} \mathrm{O}_{2} \mathrm{SNa}$, 241.1238; found, 241.1236.

HPLC analysis: Chiralpak IA, Hex/IPA $=99 / 1,0.5 \mathrm{~mL} / \mathrm{min}, 220 \mathrm{~nm} ; \mathrm{t}_{R}=18.8 \mathrm{~min}$ (minor, $S$ ), $20.0 \min$ (major, $R$ ) 
Table S1. Screening of hydrophobic cosolvents ${ }^{a}$

\begin{tabular}{|c|c|c|c|}
\hline $1 a$ & $\begin{array}{c}\mathbf{2 a} \\
\text { (1.2 equiv) }\end{array}$ & $\begin{array}{l}\text { lyst } 5 \mathrm{a}(30 \mathrm{~mol} \%) \\
\text { ), additive (5 equiv) } \\
0^{\circ} \mathrm{C}, 24 \mathrm{~h} \\
1150 \mathrm{rpm}\end{array}$ & $\prod_{\substack{0 \\
\text { aa }}}^{\mathrm{OH}}$ \\
\hline Entry & Additive & Conversion $(\%)^{b}$ & $e e(\%)^{c}$ \\
\hline 1 & toluene & 82 & 39 \\
\hline 2 & $o$-xylene & 77 & 40 \\
\hline 3 & $m$-xylene & 74 & 43 \\
\hline 4 & $p$-xylene & 77 & 41 \\
\hline 5 & chlorobenzene & 82 & 39 \\
\hline 6 & bromobenzene & 75 & 43 \\
\hline 7 & 1,2-dichlorobenzene & 79 & 41 \\
\hline 8 & $\mathrm{CH}_{2} \mathrm{Cl}_{2}$ & 82 & 31 \\
\hline 9 & diethyl ether & 69 & 56 \\
\hline 10 & diisopropyl ether & 36 & 67 \\
\hline 11 & dibuthyl ether & 50 & 66 \\
\hline 12 & dipentyl ether & 43 & 68 \\
\hline 13 & MTBE (20 equiv) & 32 & 75 \\
\hline 14 & methoxy cyclohexane & 66 & 71 \\
\hline 15 & methyl propargyl ether & 91 & 39 \\
\hline 16 & THF & 82 & 51 \\
\hline 17 & 2-methyl THF & 62 & 62 \\
\hline 18 & CPME & 60 & 74 \\
\hline 19 & CPME ( 2.5 equiv)/THF ( 2.5 equiv) & 63 & 64 \\
\hline 20 & 1,2-dimethyxy ethane & 90 & 39 \\
\hline 21 & eucalyptol & 51 & 77 \\
\hline 22 & $(R)-(+)$-limonene (20 equiv) & 26 & 71 \\
\hline 23 & $(S)-(-)$-limonene (20 equiv) & 38 & 60 \\
\hline 24 & $(+)-(\alpha)$-pinene (20 equiv) & 47 & 51 \\
\hline 25 & $(-)-(\alpha)$-pinene (20 equiv) & 32 & 53 \\
\hline
\end{tabular}

${ }^{a}$ General reaction conditions: 1a $(0.1 \mathrm{mmol}), \mathbf{2}(0.12 \mathrm{mmol})$, and catalyst $5 \mathbf{a}(30 \mathrm{~mol} \%)$ on $2.0 \mathrm{~mL}$ of media at $20{ }^{\circ} \mathrm{C}$. ${ }^{b}$ The conversion was determined by ${ }^{1} \mathrm{H}$ NMR integration. ${ }^{c}$ Enantiomeric excess $(\% e e)$ was determined by highperformance liquid chromatography (HPLC). 
Table S2. Equivalent screening

\begin{tabular}{|c|c|c|c|c|c|}
\hline & O & \multicolumn{2}{|c|}{$\begin{array}{c}\text { catalyst } 5 \mathrm{a}(30 \mathrm{~mol} \%) \\
\text { brine }(2 \mathrm{~mL}) \text {, additive } \\
20^{\circ} \mathrm{C}, 24 \mathrm{~h}\end{array}$} & & $\begin{array}{c}O \\
4 a d\end{array}$ \\
\hline Entry & Thiol & Additive & Conversion (\%) & $e e(\%)$ & condition \\
\hline 1 & $t$-BuSH (2 equiv) & eucalyptol (5 equiv) & 32 & 89 & on-water \\
\hline 2 & $t$-BuSH ( 6 equiv) & eucalyptol (5 equiv) & 47 & 91 & on-water \\
\hline 3 & $t$-BuSH (10 equiv) & eucalyptol (5 equiv) & 61 & 90 & on-water \\
\hline 4 & $t$-BuSH (20 equiv) & eucalyptol (5 equiv) & 64 & 90 & on-water \\
\hline 5 & $t$-BuSH (6 equiv) & - & 68 & 70 & on-water \\
\hline 6 & $t$-BuSH (10 equiv) & - & 79 & 73 & on-water \\
\hline 7 & $t$-BuSH (20 equiv) & - & 81 & 78 & on-water \\
\hline
\end{tabular}


Table S3. Effect of droplet size under the biphasic microfluidic conditions

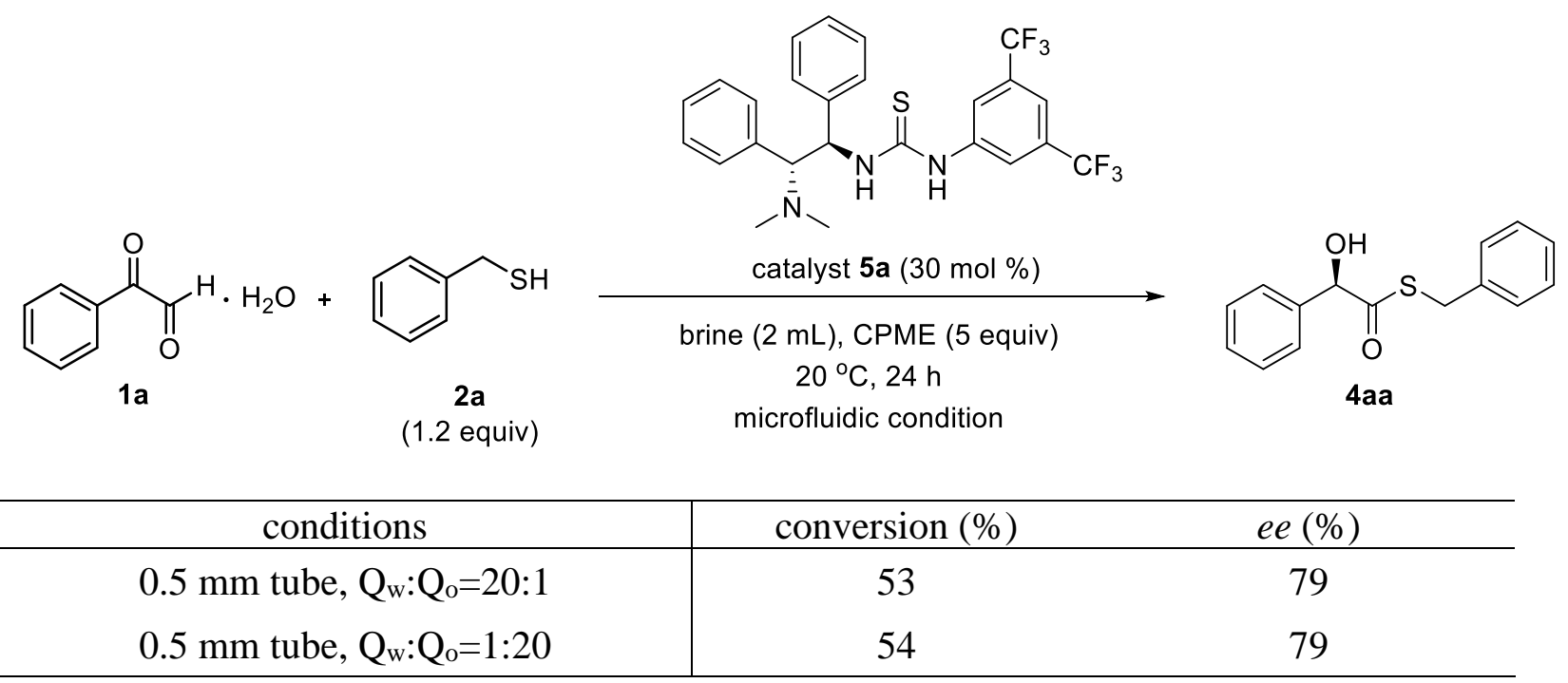


Table S4. High-pressure experiments for the determination of activation volume

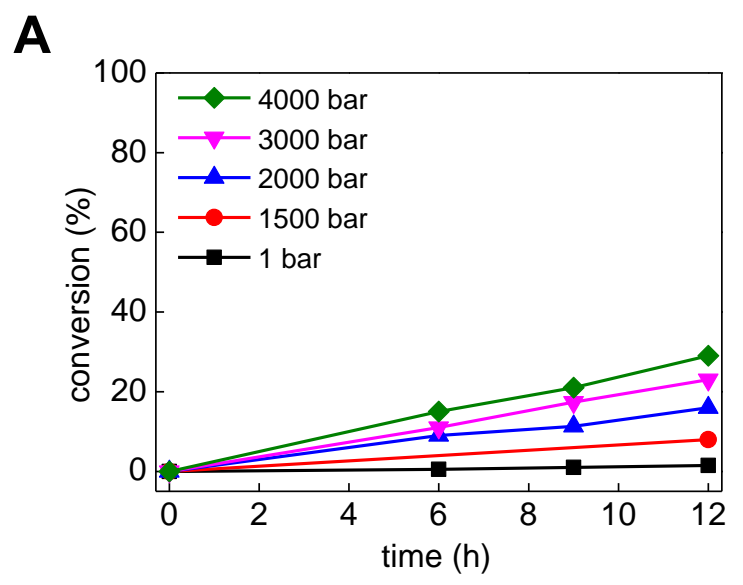

B

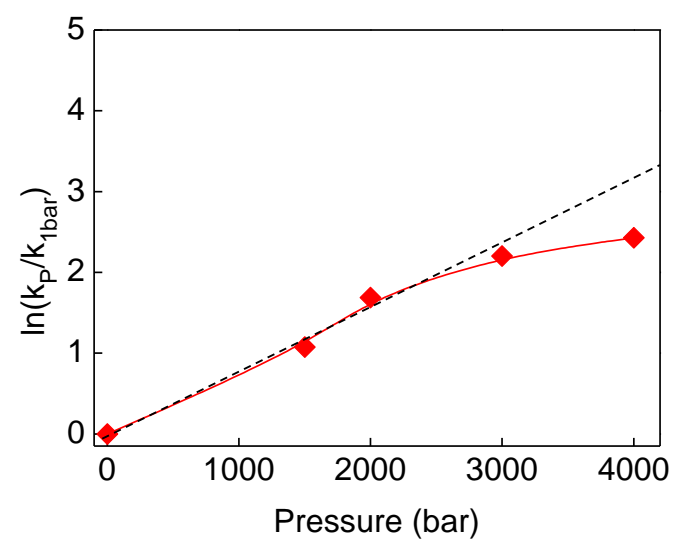

\begin{tabular}{ccccccc}
\hline Pressure & \multicolumn{5}{c}{ Conversion (\%) } & \multirow{2}{*}{$\mathrm{k}_{\text {rel }}$} \\
\cline { 2 - 6 }$(\mathrm{b} a \mathrm{r})$ & $6 \mathrm{~h}$ & $9 \mathrm{~h}$ & $12 \mathrm{~h}$ & $24 \mathrm{~h}$ & $36 \mathrm{~h}$ & \\
\hline 1 & $<1$ & 1 & - & 2 & - & 1 \\
1500 & - & - & 8 & 15 & 22 & 2.93 \\
2000 & 9 & 11 & 16 & - & - & 5.40 \\
3000 & 11 & 17 & 23 & - & - & 9.05 \\
4000 & 15 & 21 & 29 & - & - & 11.33 \\
\hline
\end{tabular}

(A) Effect of pressure on the reaction rate. $\mathrm{k}_{\text {rel }}=1.0(1 \mathrm{bar}), \mathrm{k}_{\text {rel }}=2.93(1.5 \mathrm{kbar}), \mathrm{k}_{\mathrm{rel}}=5.40$ (2 $\mathrm{kbar}), \mathrm{k}_{\mathrm{rel}}=9.05(3 \mathrm{kbar}), \mathrm{k}_{\mathrm{rel}}=11.33$ (4 kbar) (B) Determination of activation volume according to the equation $\ln \left(\mathrm{k}_{\mathrm{P}} / \mathrm{k}_{1 \mathrm{bar}}\right)=-\left(\Delta \Delta \mathrm{V}^{\ddagger} / \mathrm{RT}\right)(\mathrm{P}-1)+\mathrm{C}$.

*Comment 1: The kinetic analysis is proposed in terms of the formation of the thioester product 4aa, which leads to the same results as the analysis for the consumption of starting material.

*Comment 2: Plotting In $\left(\mathrm{k}_{P}-\mathrm{k}_{1 \mathrm{bar}}\right)$ vs. $(P-1)$ shows a typical curve with a linear dependence in the low-pressure region, leveling off at higher pressures. This may be due to the increased viscosity of the solvent at higher pressures. 
Table S5. Study of the effect of catalyst optical purity on product optical purity ${ }^{a}$

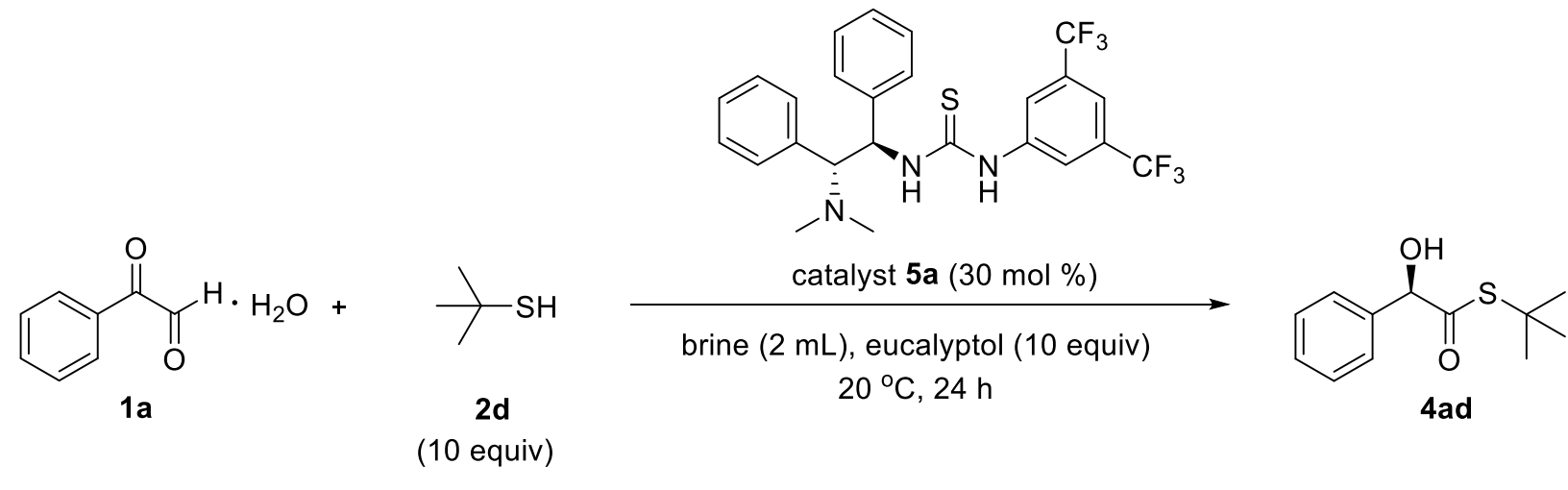

\begin{tabular}{|c|c|c|c|c|c|c|}
\hline$e e$ of catalyst $\mathbf{5 a}[\%]$ & 3 & 23 & 42 & 60 & 81 & 100 \\
\hline$e e$ of product $\mathbf{4 a d}[\%]$ & 2 & 21 & 38 & 55 & 74 & 91 \\
\hline
\end{tabular}

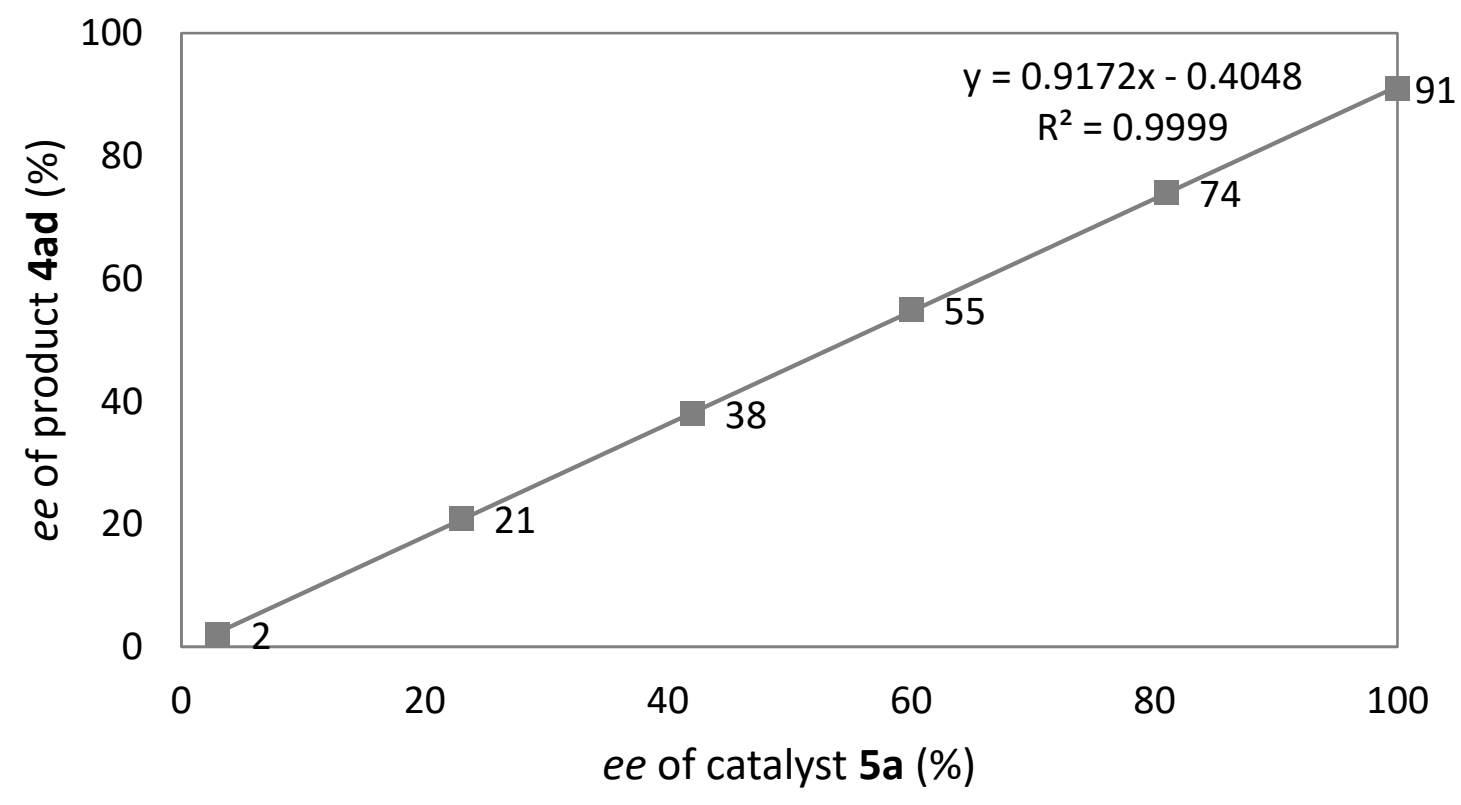


Figure S1. Thiol screening

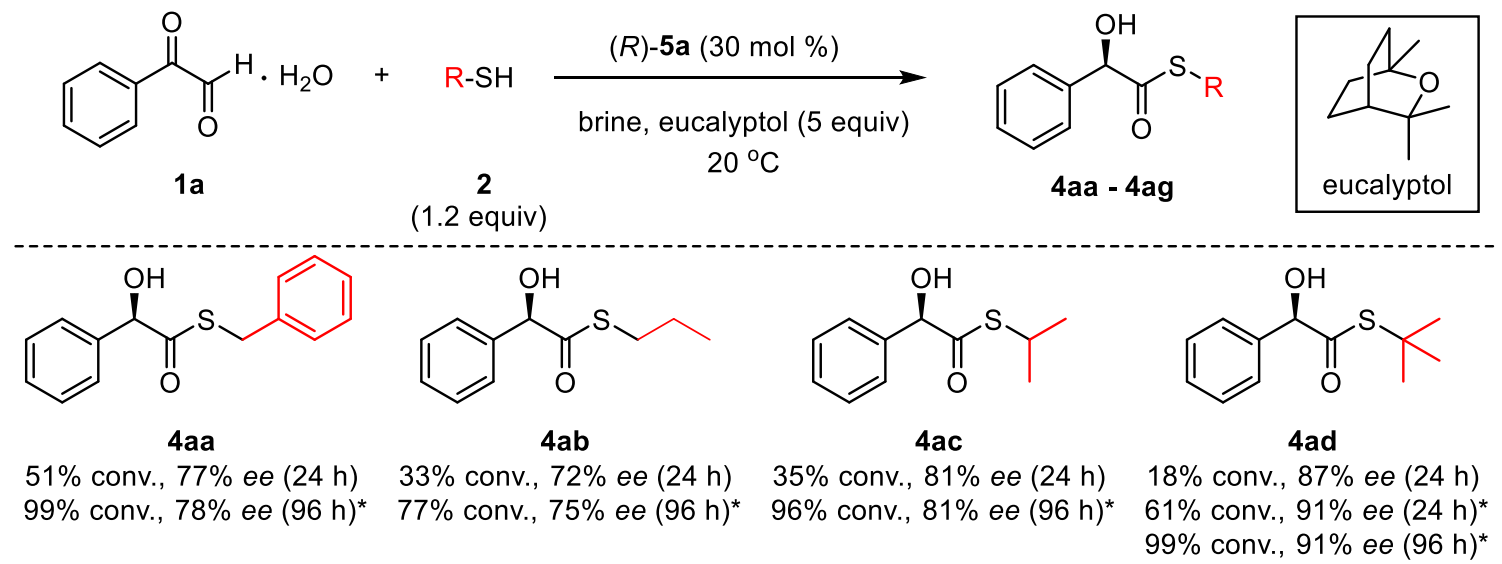

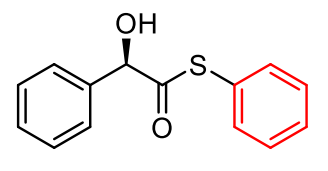

4ae

$24 \%$ conv., $36 \%$ ee $(24 \mathrm{~h})$

$99 \%$ conv., $37 \%$ ee $(96 \mathrm{~h})^{*}$

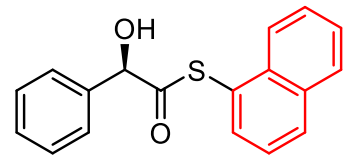

4 af

$24 \%$ conv., $30 \%$ ee $(24 \mathrm{~h})$ $99 \%$ conv., $31 \%$ ee $(96 \mathrm{~h})$ *

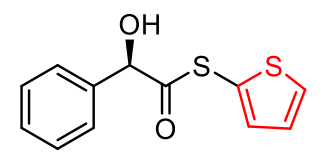

4ag

$>2 \%$ conv., ee: n.d. (24 h) $19 \%$ conv., $11 \%$ ee $(96 \mathrm{~h})^{*}$

*10 equiv of R-SH was used.

*Comments: Regardless of the degree of substitution, alkyl thiols were found to serve as suitable GSH surrogates in terms of enantioselectivity. However, aromatic and heteroaromatic thiols were markedly inferior for enantioselectivity. 
Figure S2. Schematic representation of the double-subtraction procedure of IR spectra
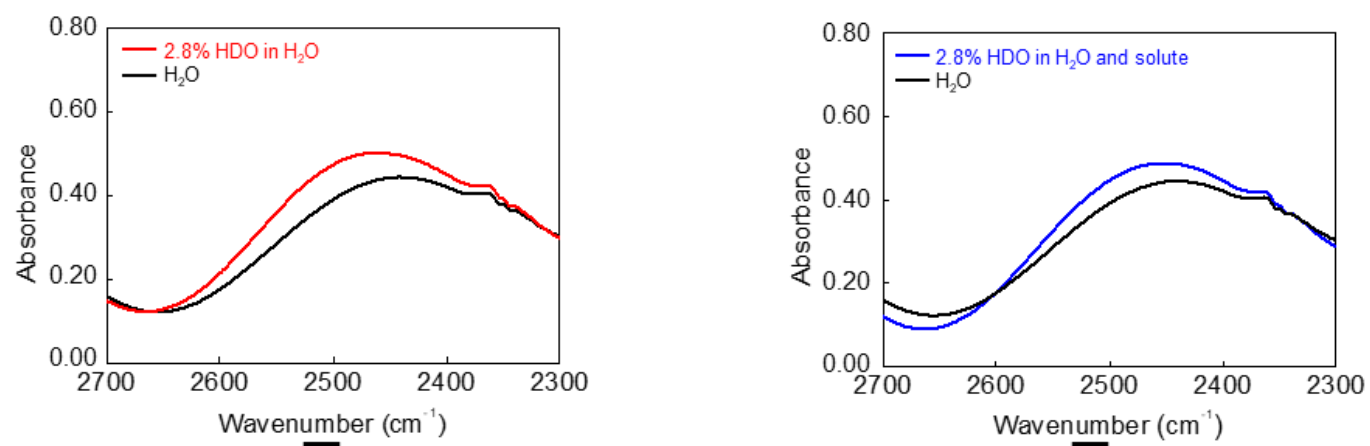

\section{Subtraction of $\mathrm{H}_{2} \mathrm{O}$ spectrum}
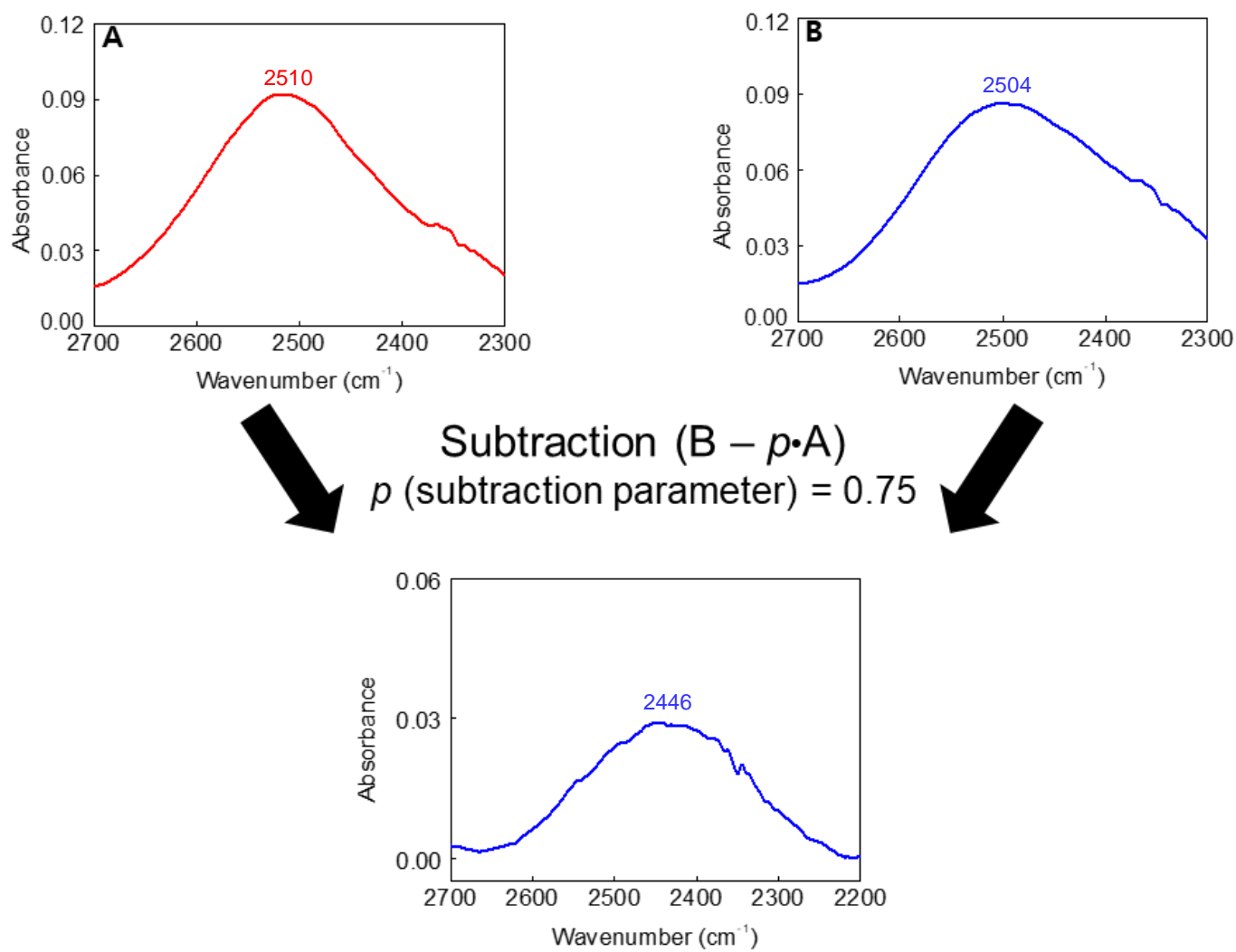

*The subtraction procedure developed by Lindgren and coworkers was used to determine the hydrogen bond strength of water molecules near the hydrophobic reaction mixture under onwater conditions. ${ }^{5}$

*Solutions of $2.8 \%$ (by volume) $\mathrm{HDO}$ in $\mathrm{H}_{2} \mathrm{O}$ were used for all IR measurements. The solution was prepared by mixing $1.4 \% \mathrm{D}_{2} \mathrm{O}$ and $98.6 \% \mathrm{H}_{2} \mathrm{O}$. A demountable flow cell (Specac, $\mathrm{ZnSe}$ window, GS20586) was used as a sample cell and deuterated triglycine sulphate (DTGS) as a detector. The spectra were collected in the transmission mode with a resolution of $4 \mathrm{~cm}^{-1}$. Thirty two interferograms were measured and apodized using the Gapp-Henzel function. The aperture of the IR beam was set to $2 \mathrm{~mm}$. Sample was injected into the cavity generated by $6 \mu \mathrm{m}$ thick Mylar spacers between the ZnSe windows mounted in the flow cell. OPUS software (Ver. 6.5) was used for subtraction of spectra. 
Figure S3. Isotope experiment with $\left[1-^{2} \mathrm{H}\right]$-phenylglyoxal

A

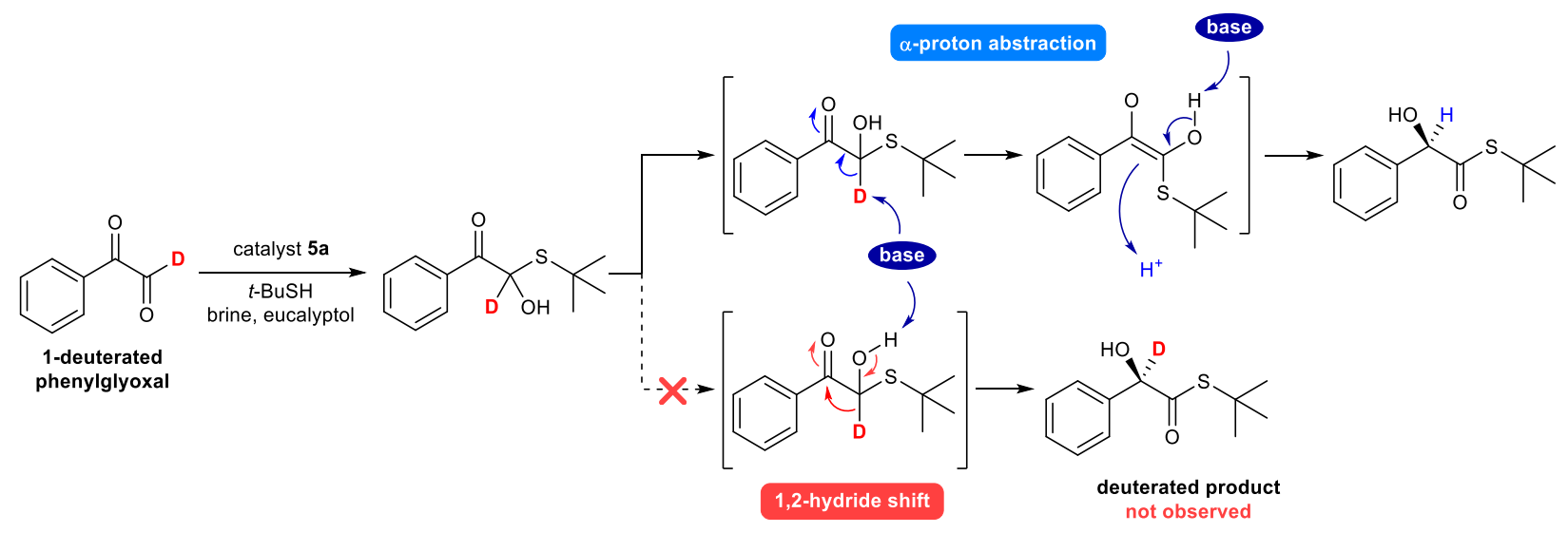

B

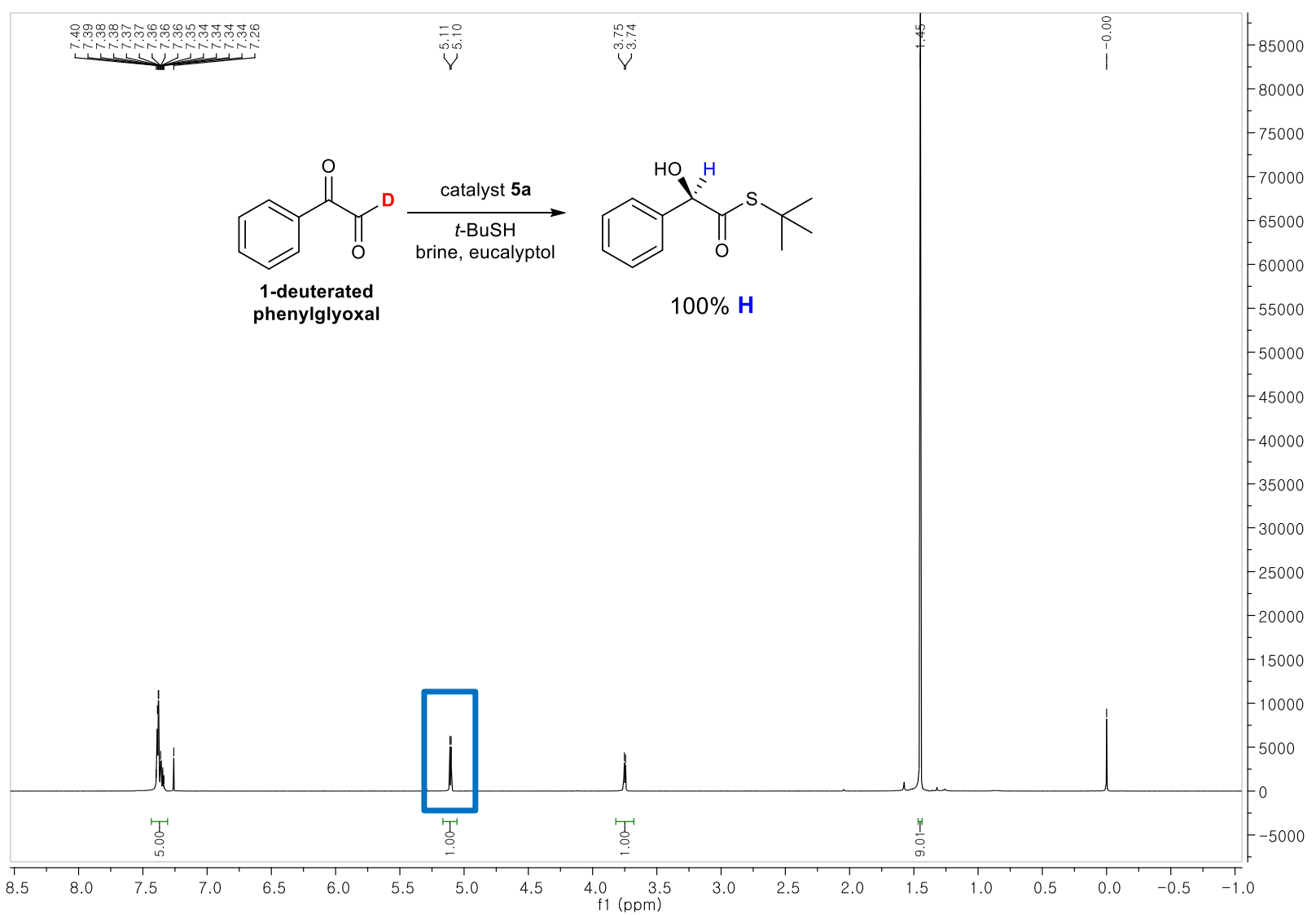

(A) Two possible mechanisms. (B) NMR spectrum of the isotope experiment with deuterated-phenylglyoxal. The reaction was performed with $\left[1-{ }^{2} \mathrm{H}\right]$-phenylglyoxal $(0.1 \mathrm{mmol}), \mathbf{2 d}(1 \mathrm{mmol})$, and catalyst $\mathbf{5 a}(30 \mathrm{~mol} \%)$ on brine $(2.0 \mathrm{~mL})$ and eucalyptol $(1 \mathrm{mmol})$ at $20^{\circ} \mathrm{C}$. 
Figure S4. Study of the rate determining step for isomerization of hemithioacetals
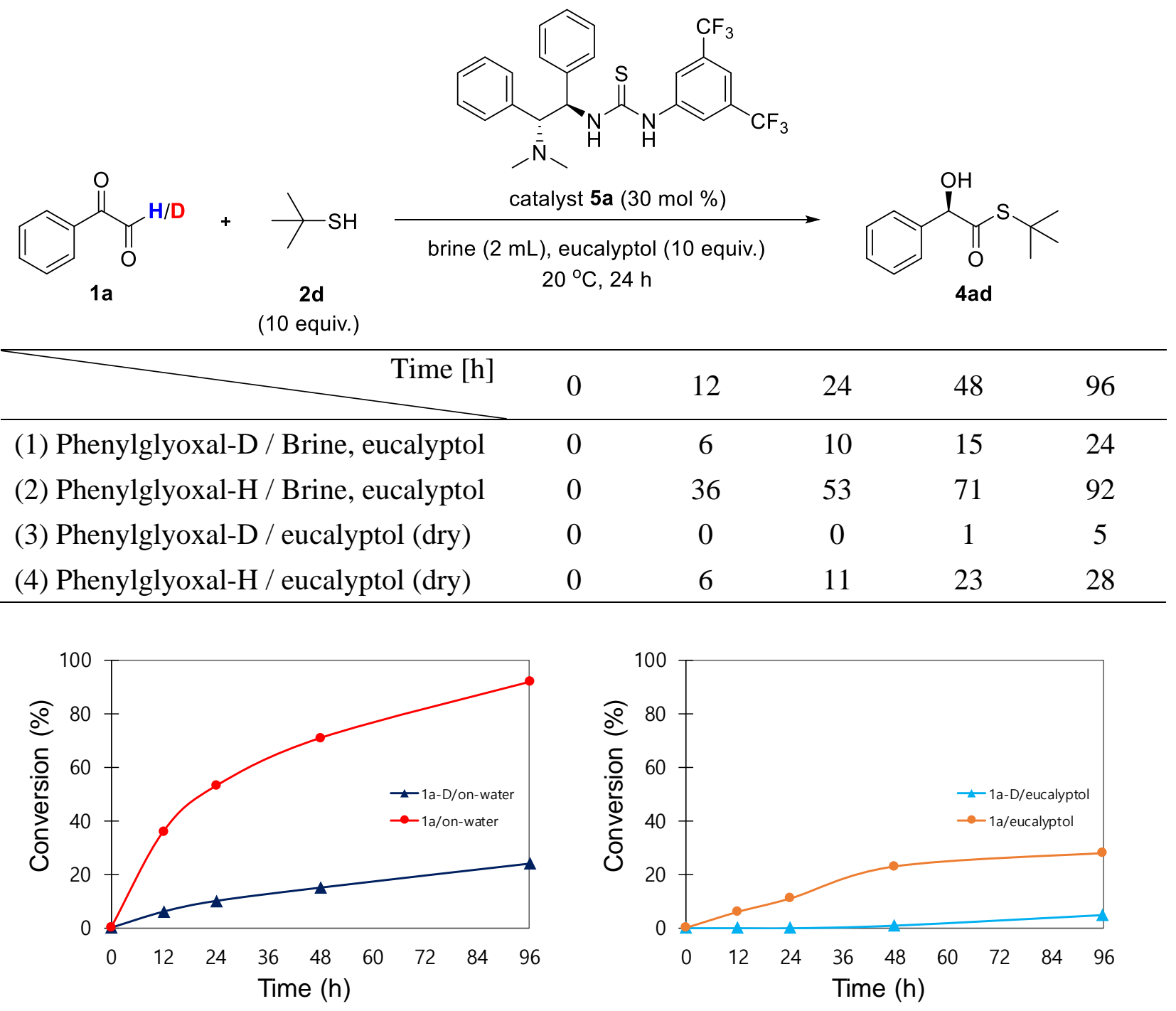


\section{NMR spectra}

NMR Spectra of catalysts

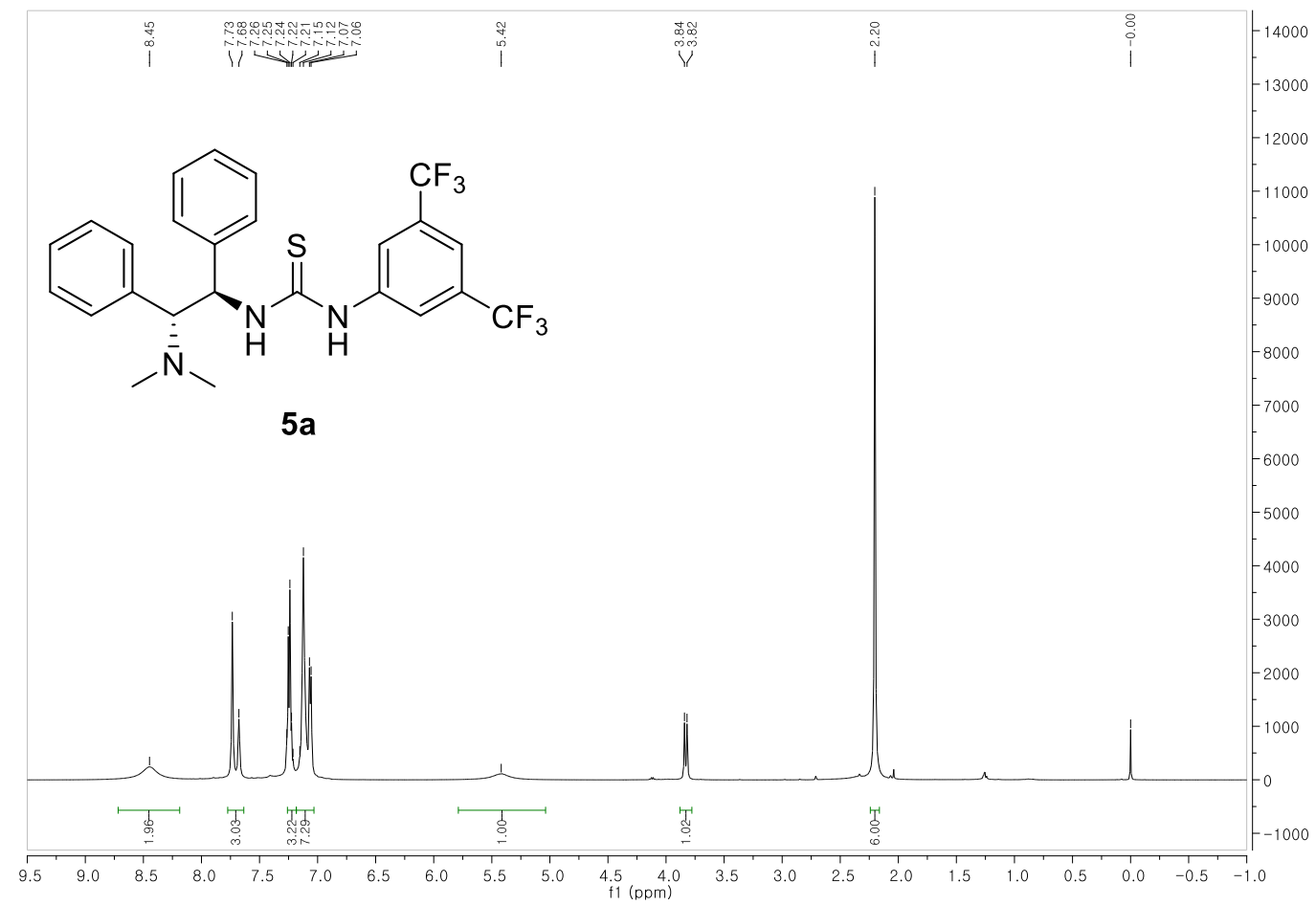

${ }^{1} \mathrm{H}$ NMR spectra of $\mathbf{5 a}$

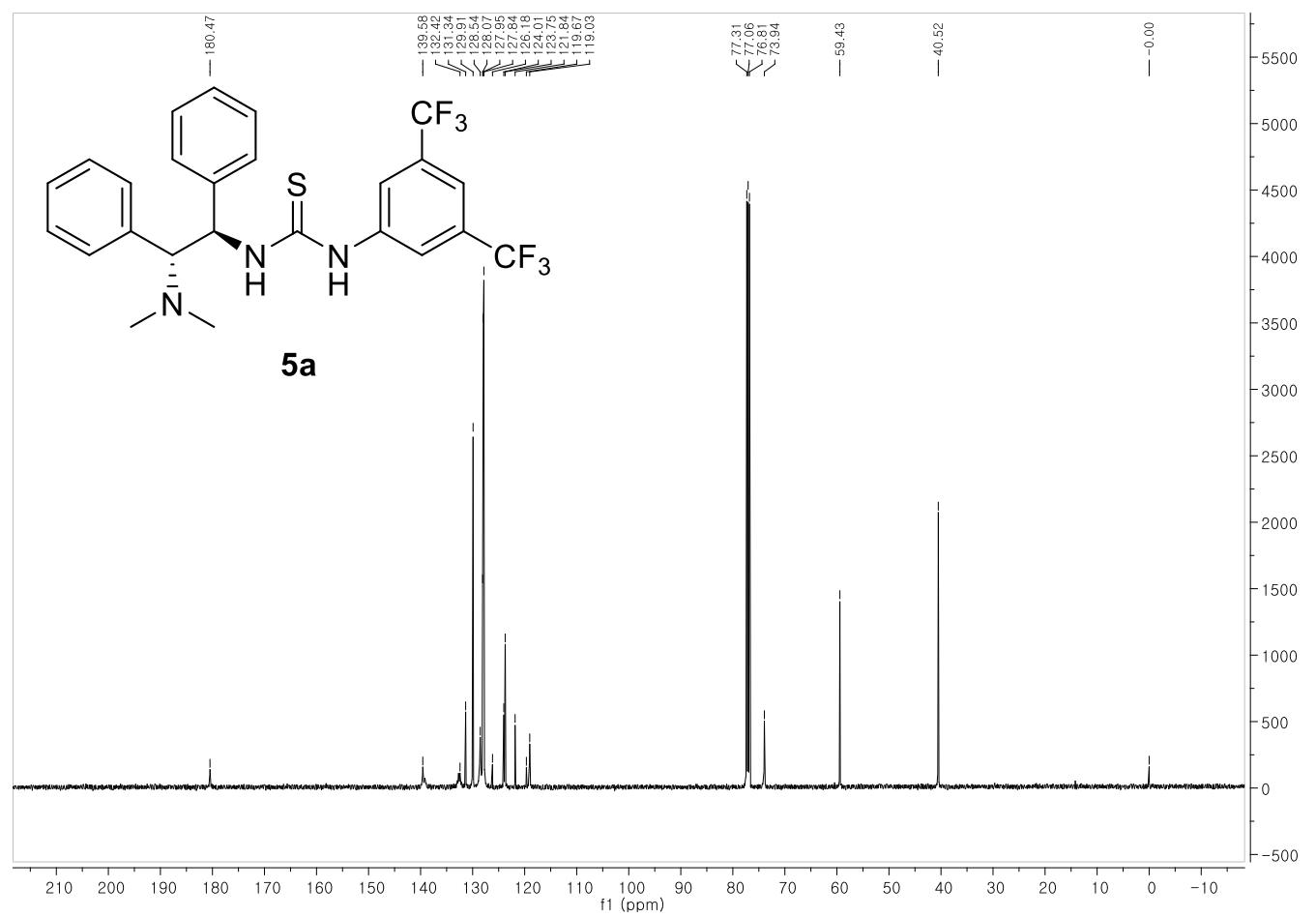

${ }^{13} \mathrm{C}$ NMR spectra of $\mathbf{5 a}$ 


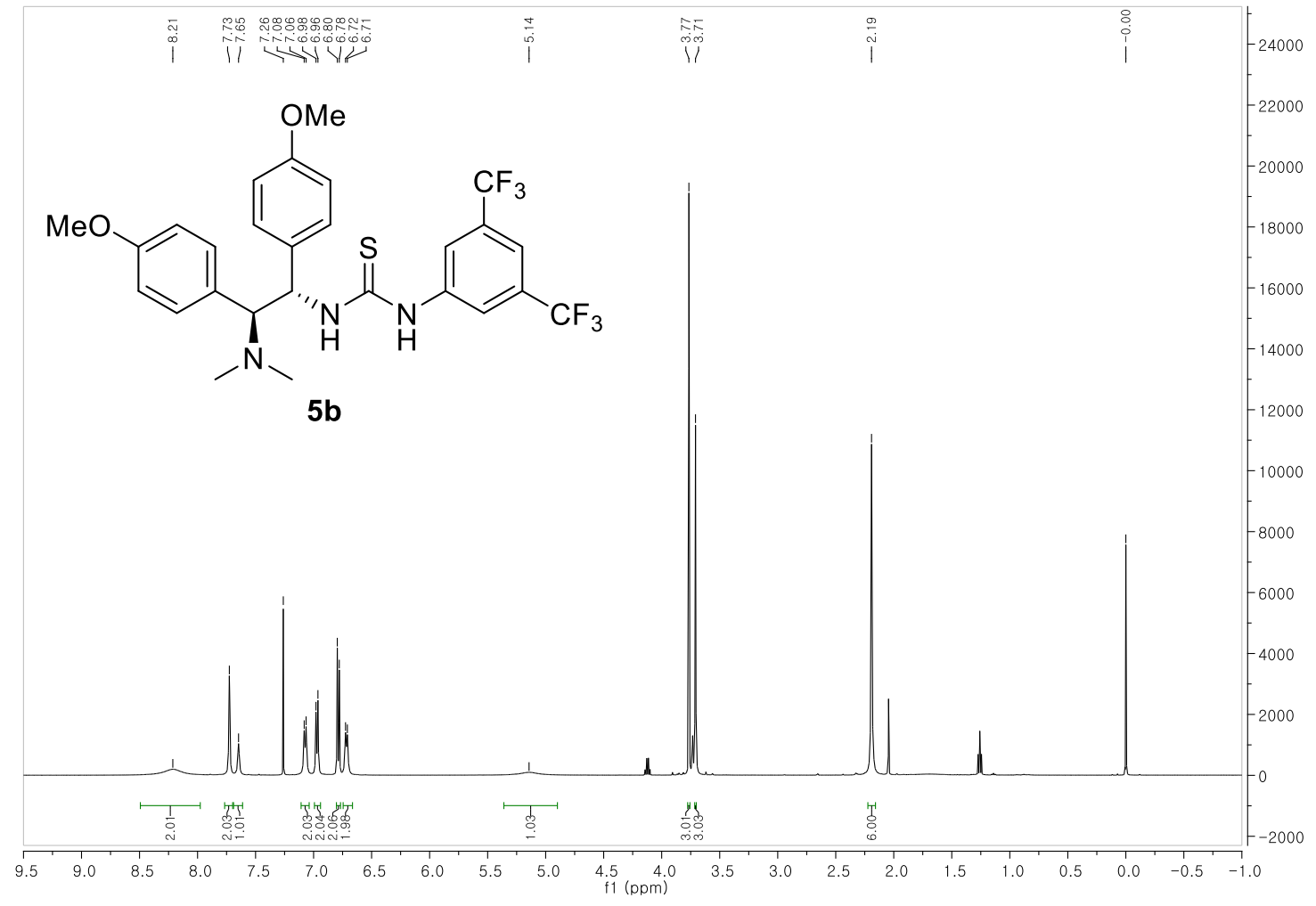

${ }^{1} \mathrm{H}$ NMR spectra of $\mathbf{5 b}$

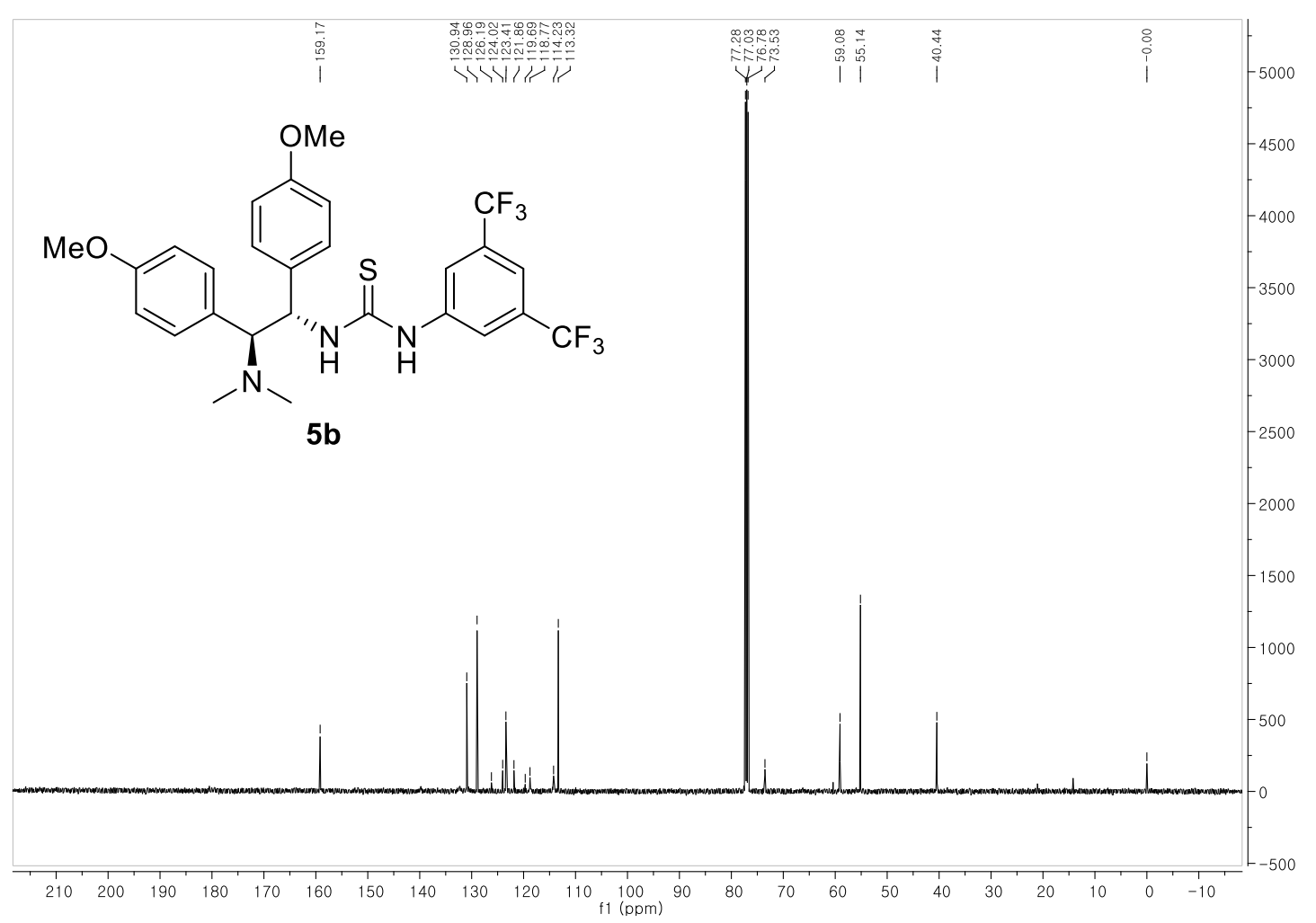

${ }^{13} \mathrm{C}$ NMR spectra of $\mathbf{5 b}$ 
NMR spectra of products 4

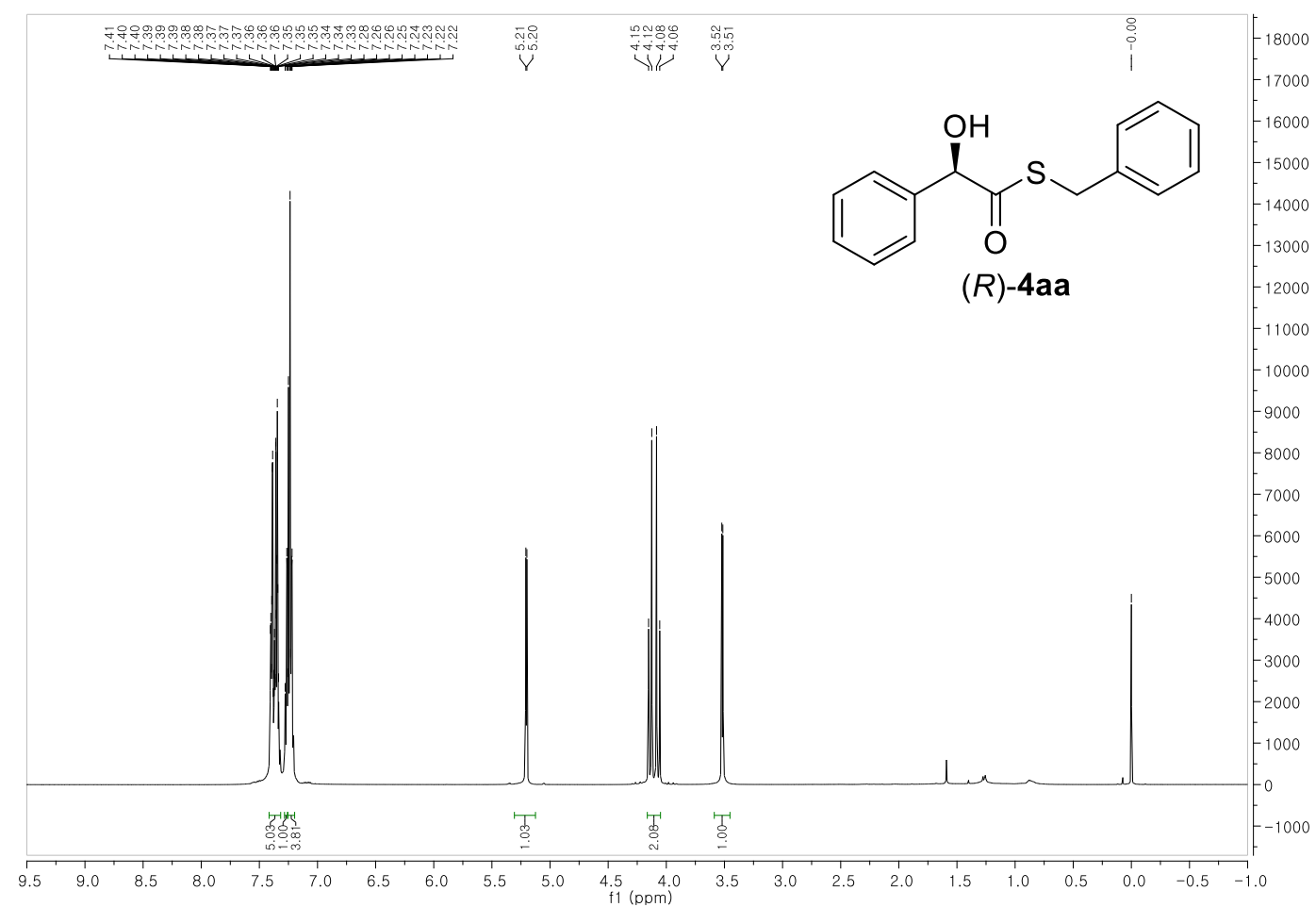

${ }^{1} \mathrm{H}$ NMR spectra of $\mathbf{4 a a}$

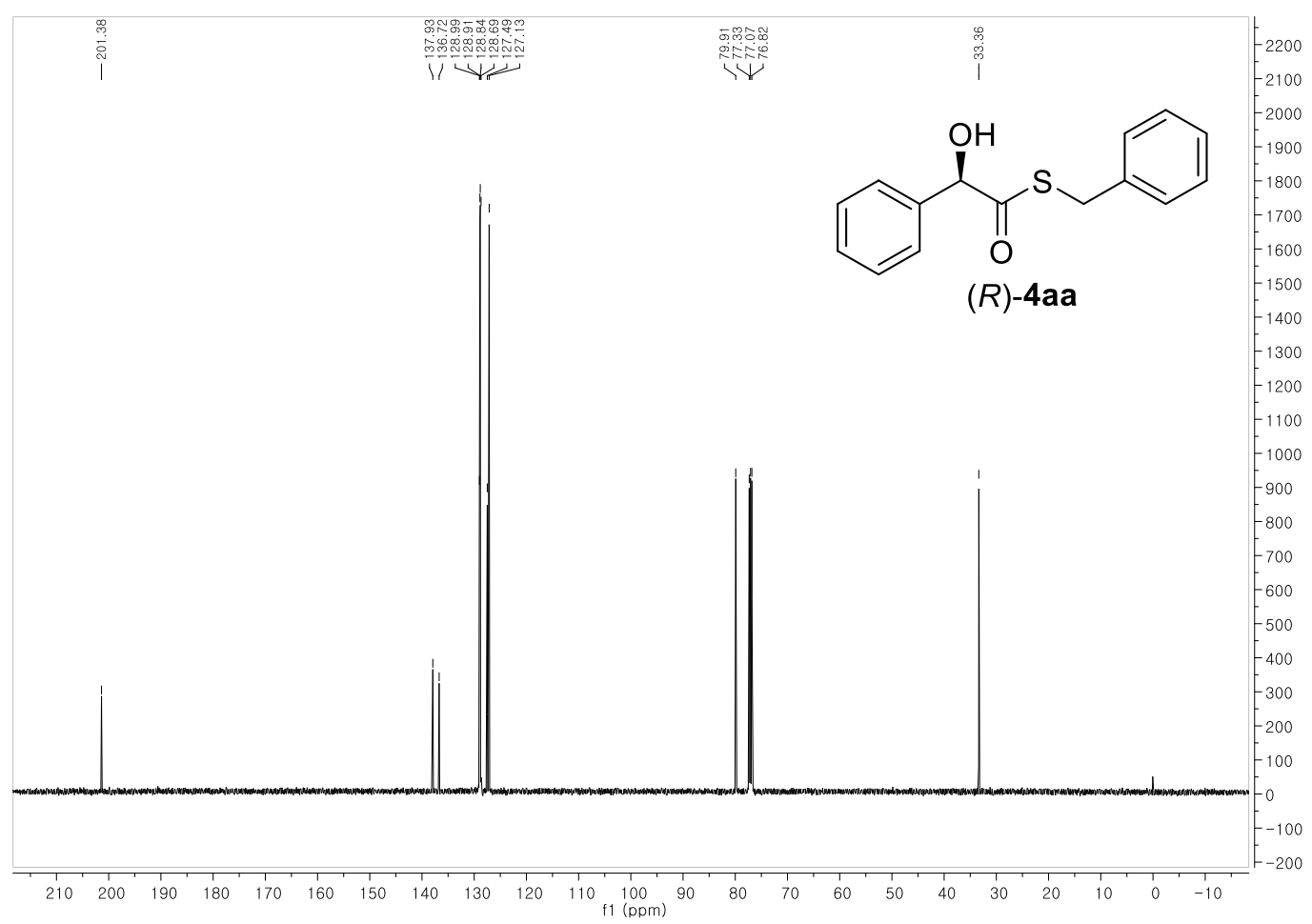

${ }^{13} \mathrm{C}$ NMR spectra of $\mathbf{4 a a}$ 


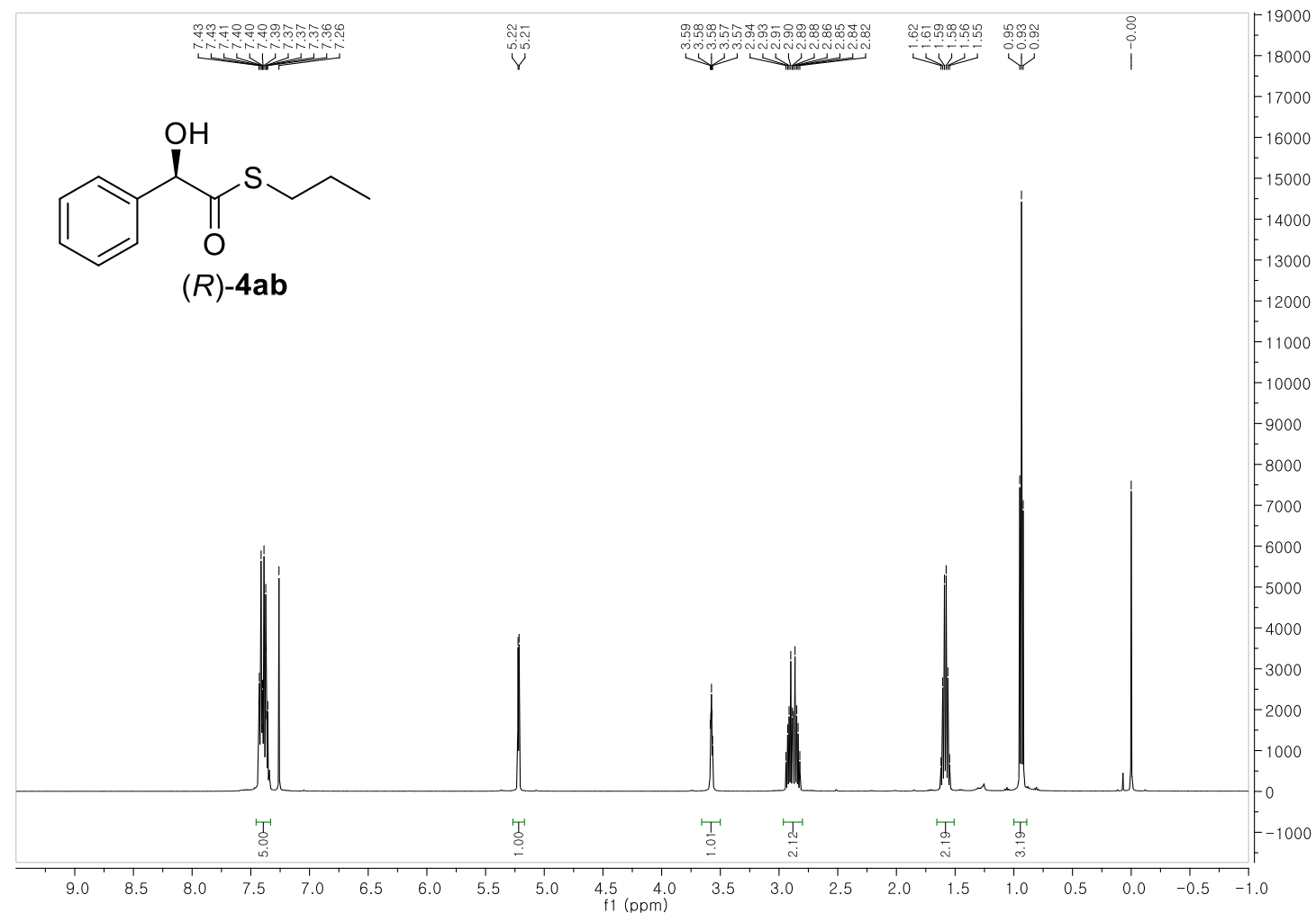

${ }^{1} \mathrm{H}$ NMR spectra of $\mathbf{4 a b}$

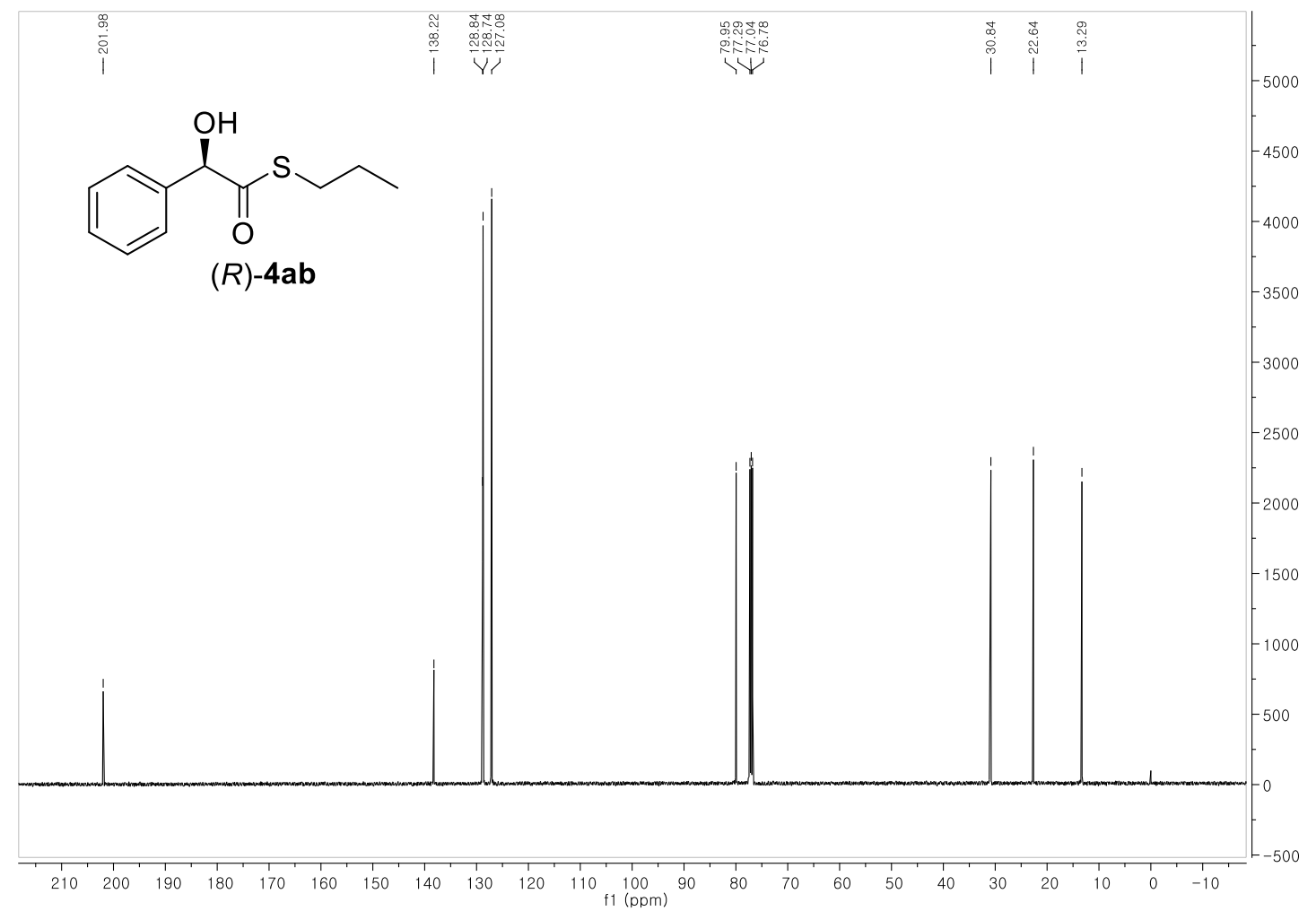

${ }^{13} \mathrm{C}$ NMR spectra of $\mathbf{4 a b}$ 


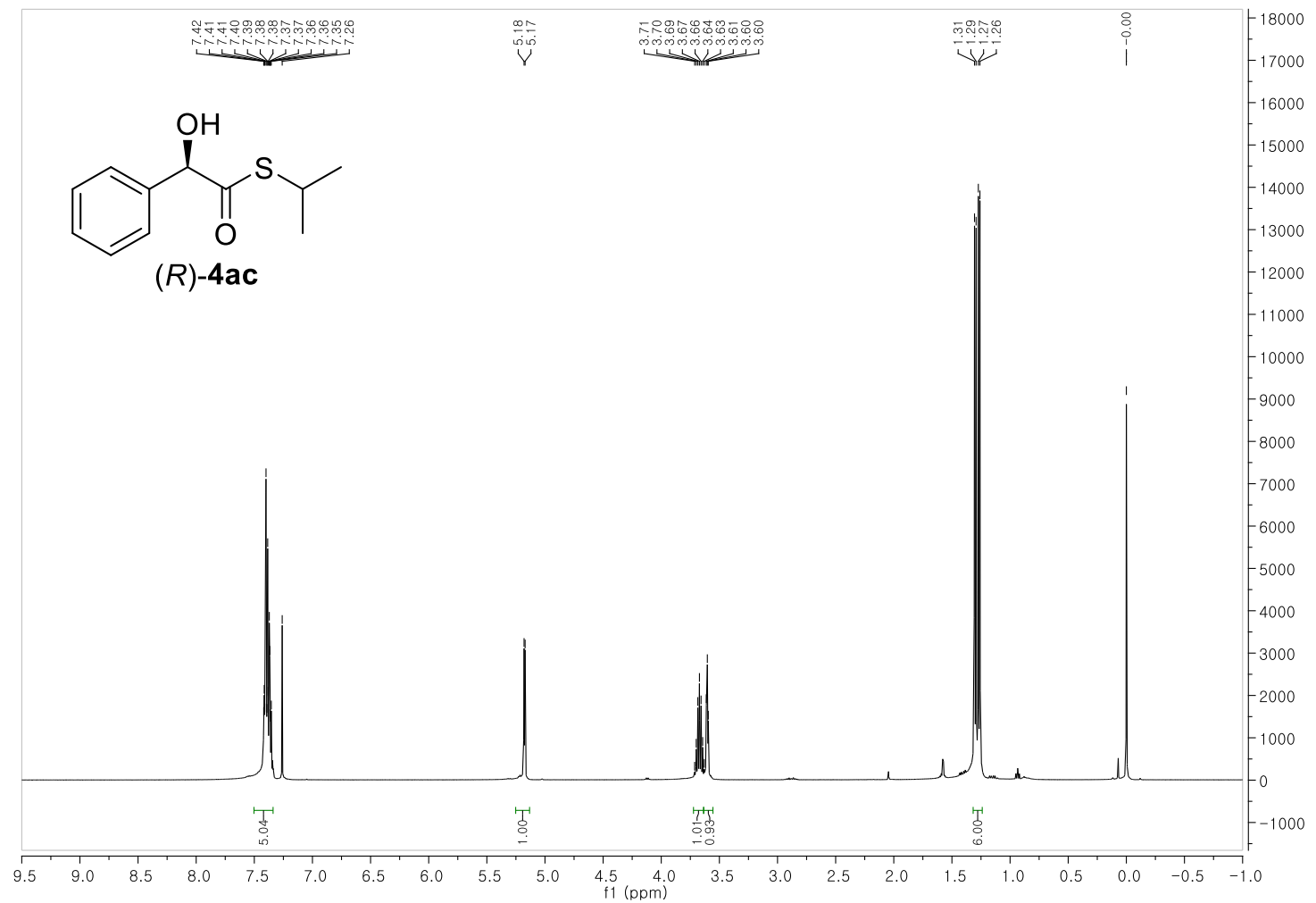

${ }^{1} \mathrm{H}$ NMR spectra of $4 \mathbf{a c}$

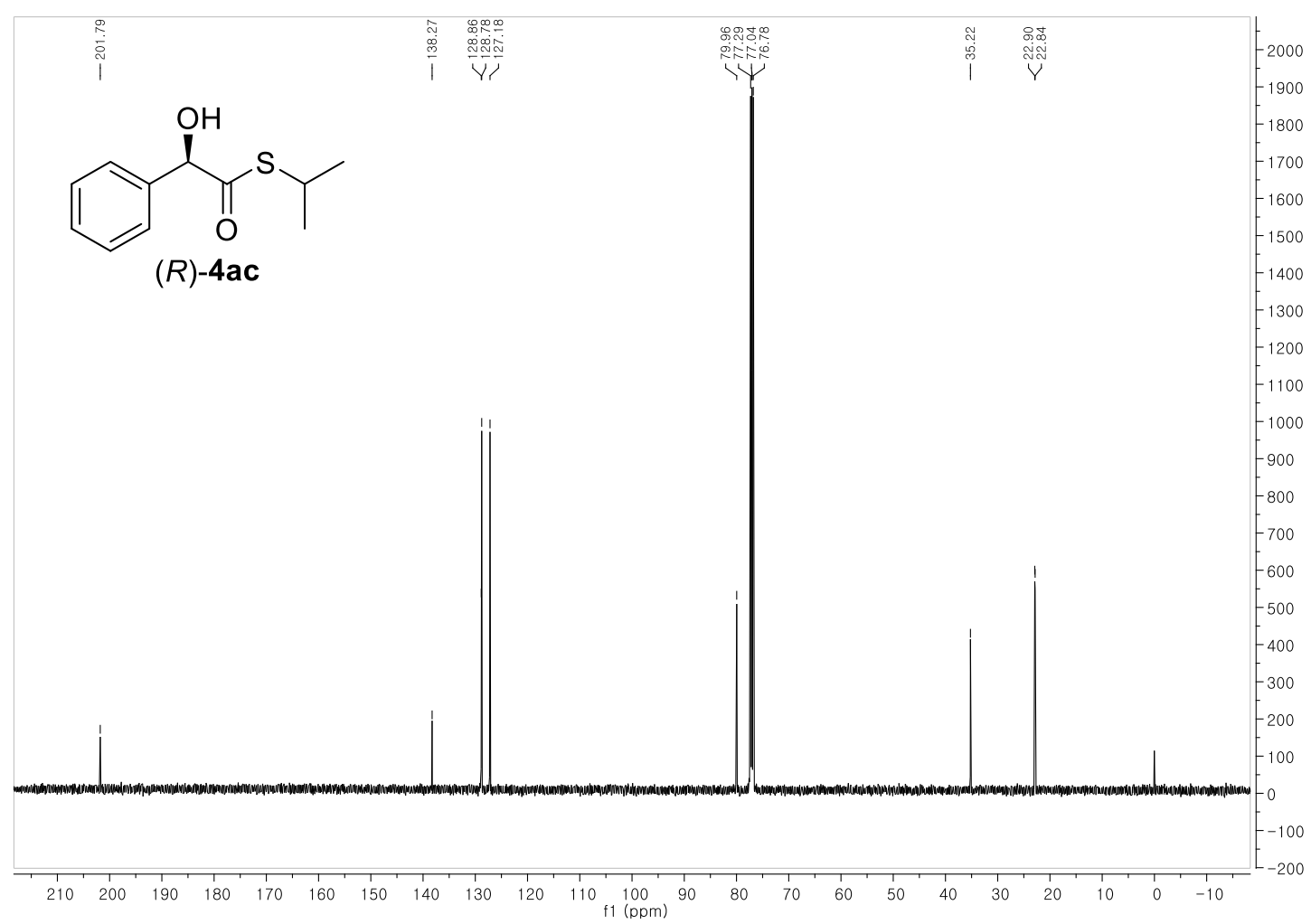

${ }^{13} \mathrm{C}$ NMR spectra of $\mathbf{4 a c}$ 


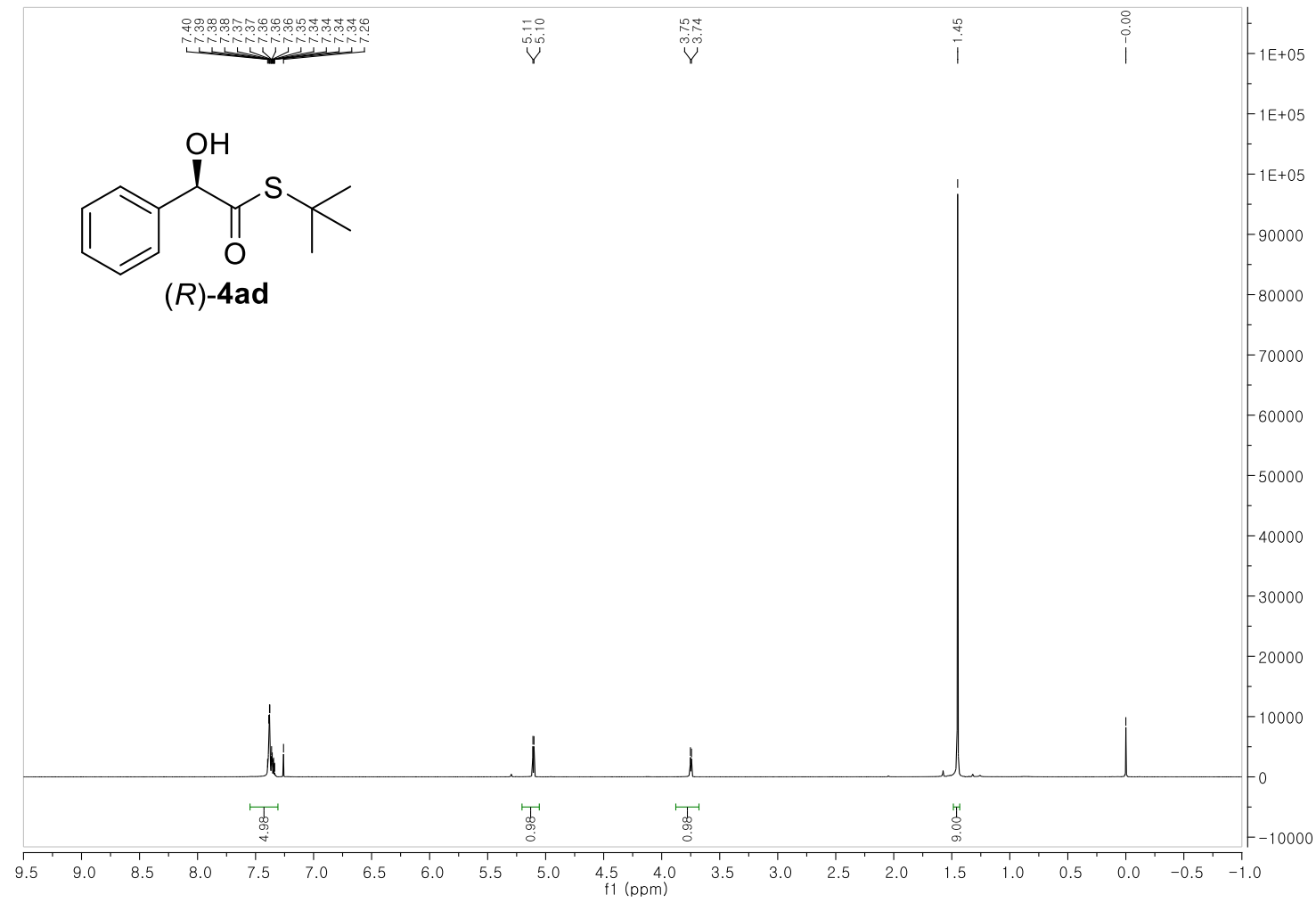

${ }^{1} \mathrm{H}$ NMR spectra of $\mathbf{4 a d}$

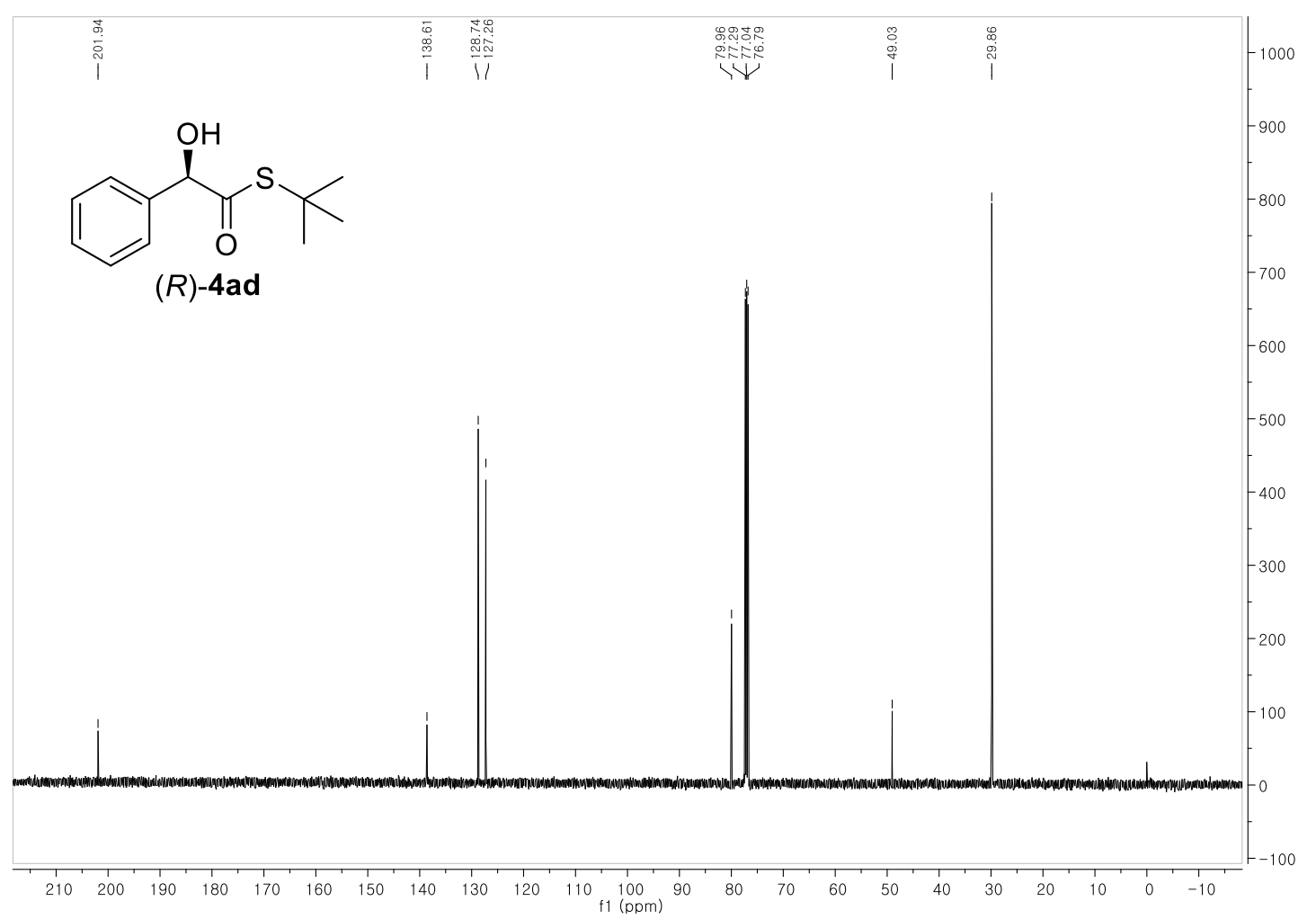

${ }^{13} \mathrm{C}$ NMR spectra of $\mathbf{4 a d}$ 


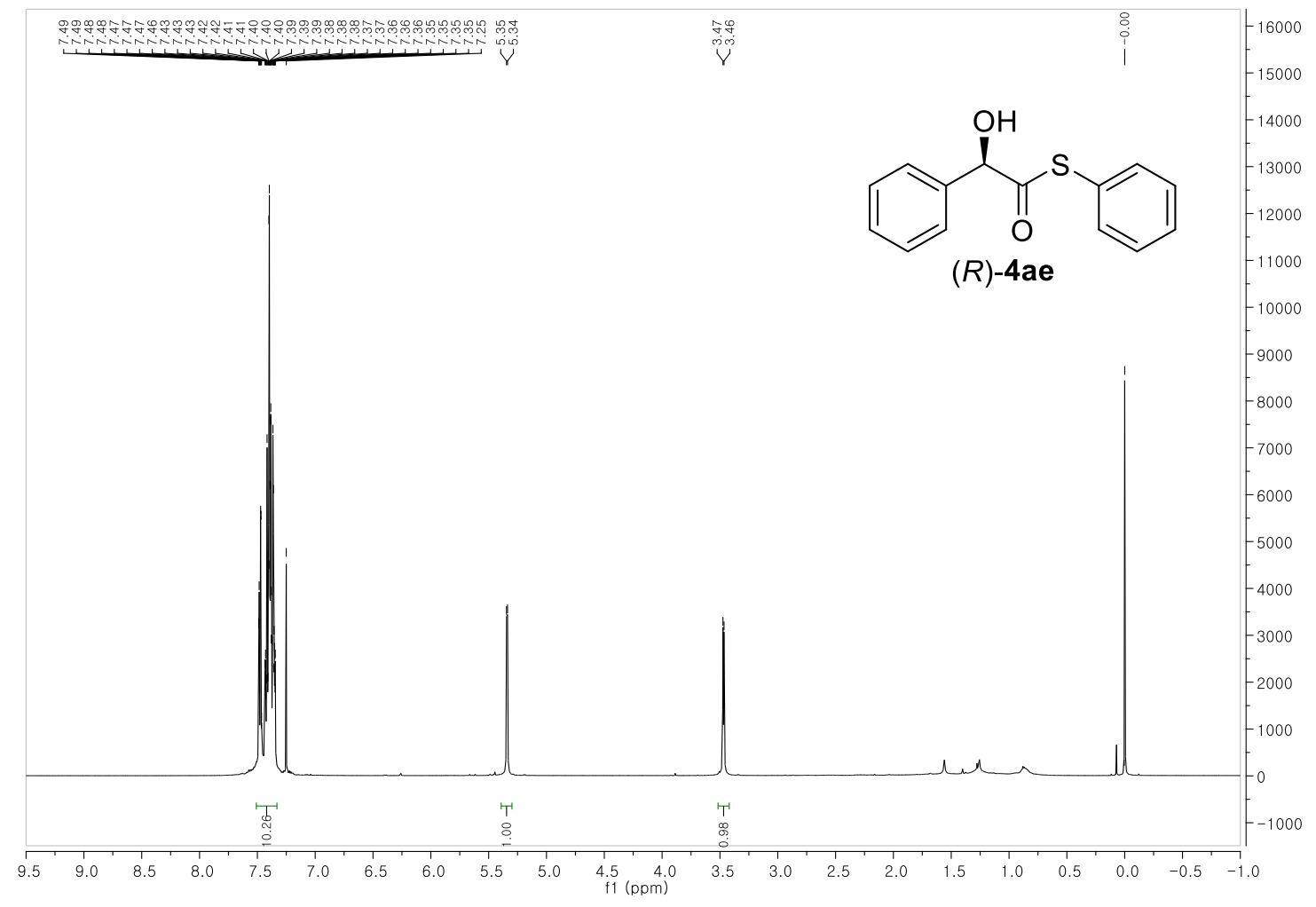

${ }^{1} \mathrm{H}$ NMR spectra of 4ae

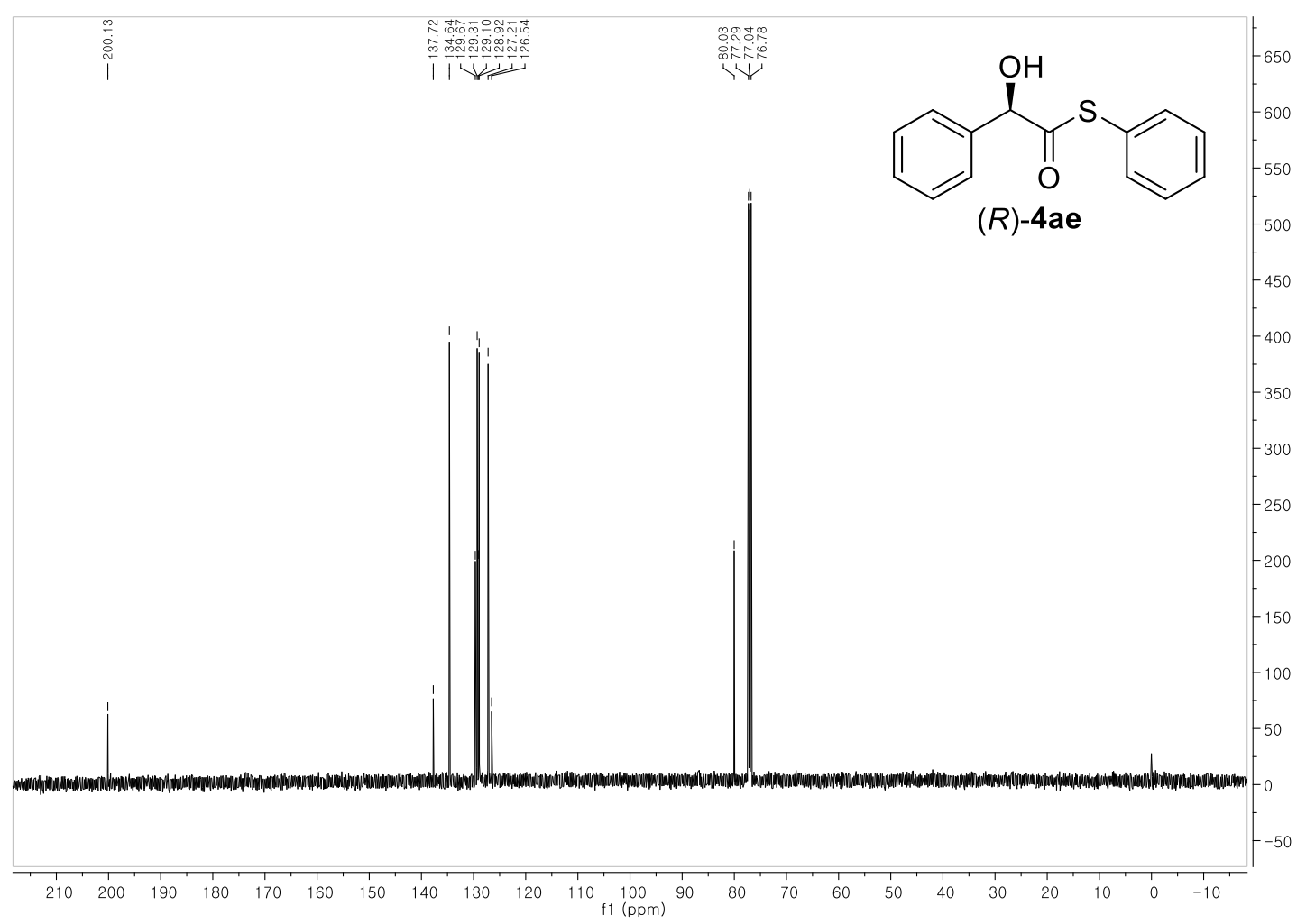

${ }^{13} \mathrm{C}$ NMR spectra of $4 \mathbf{a e}$ 


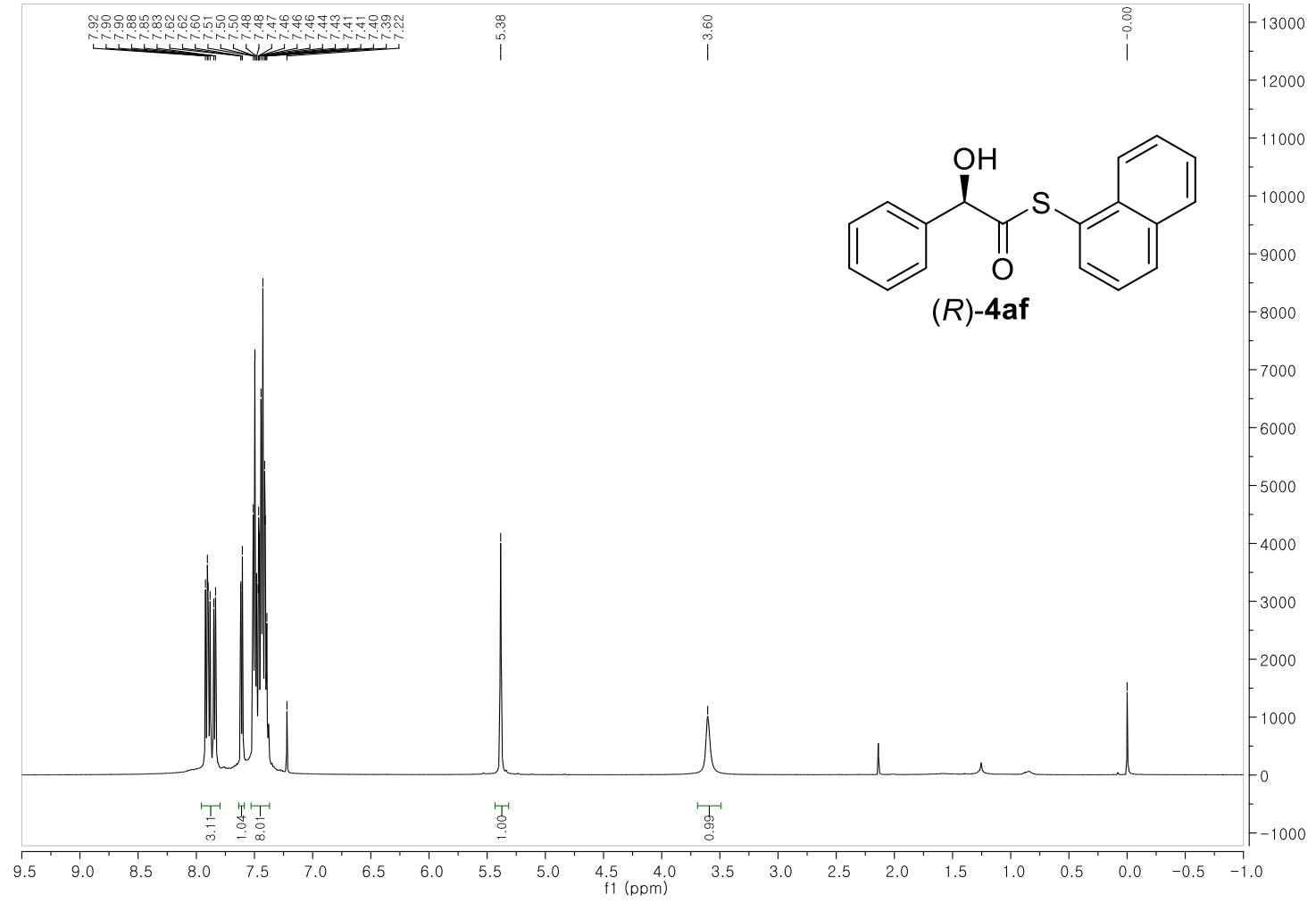

${ }^{1} \mathrm{H}$ NMR spectra of $\mathbf{4 a f}$

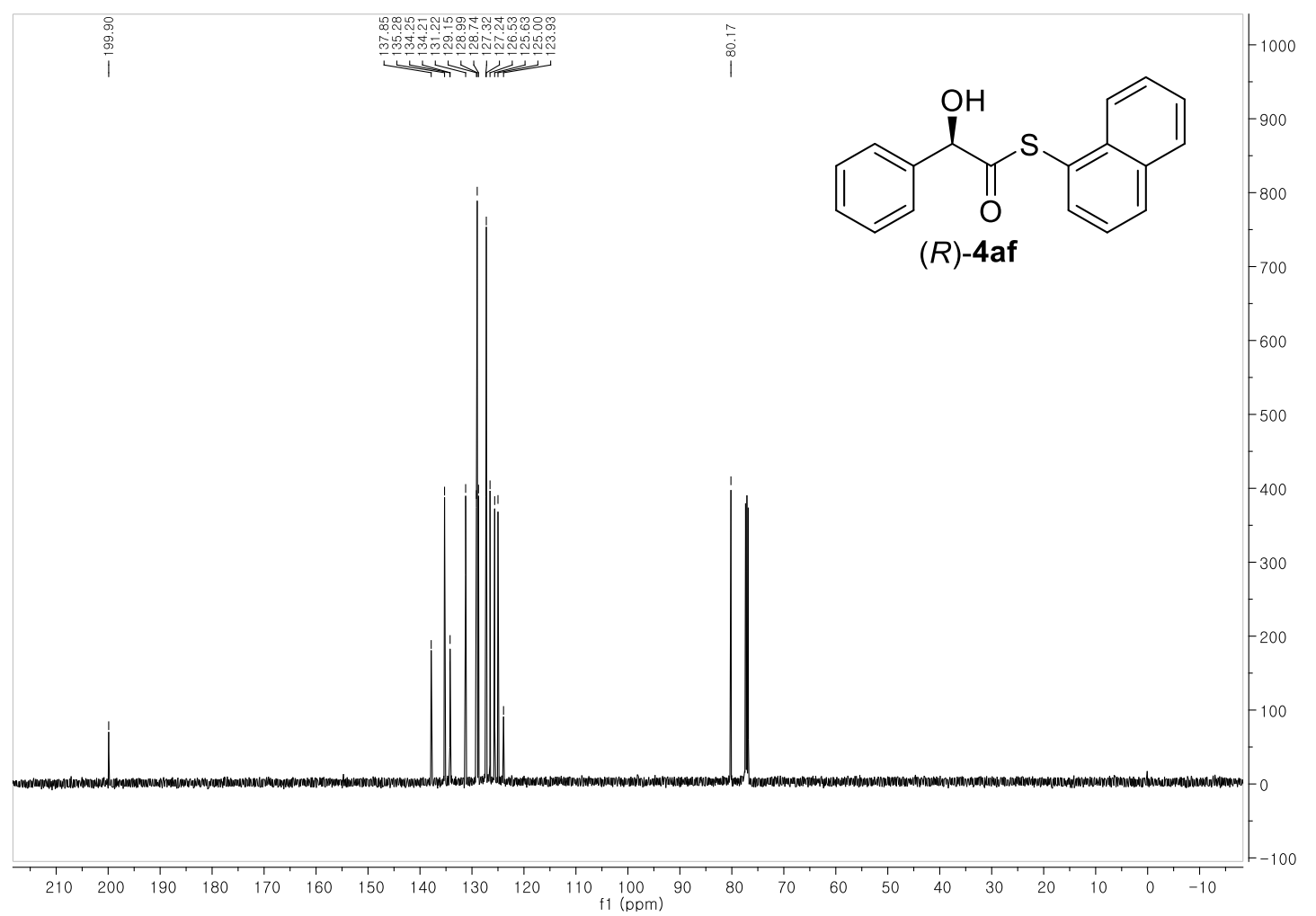

${ }^{13} \mathrm{C}$ NMR spectra of $\mathbf{4 a f}$ 


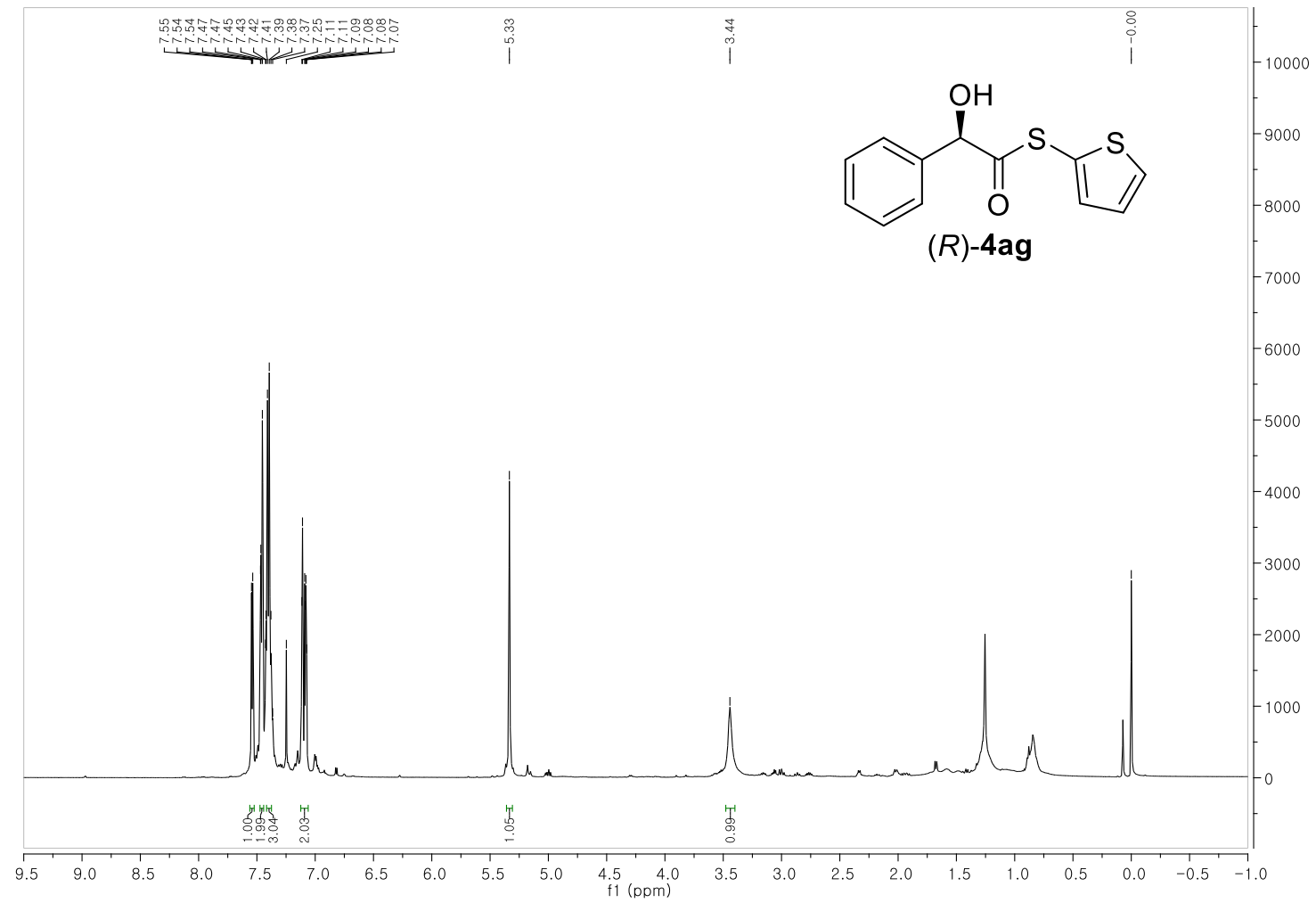

\section{${ }^{1} \mathrm{H}$ NMR spectra of $\mathbf{4 a g}$}

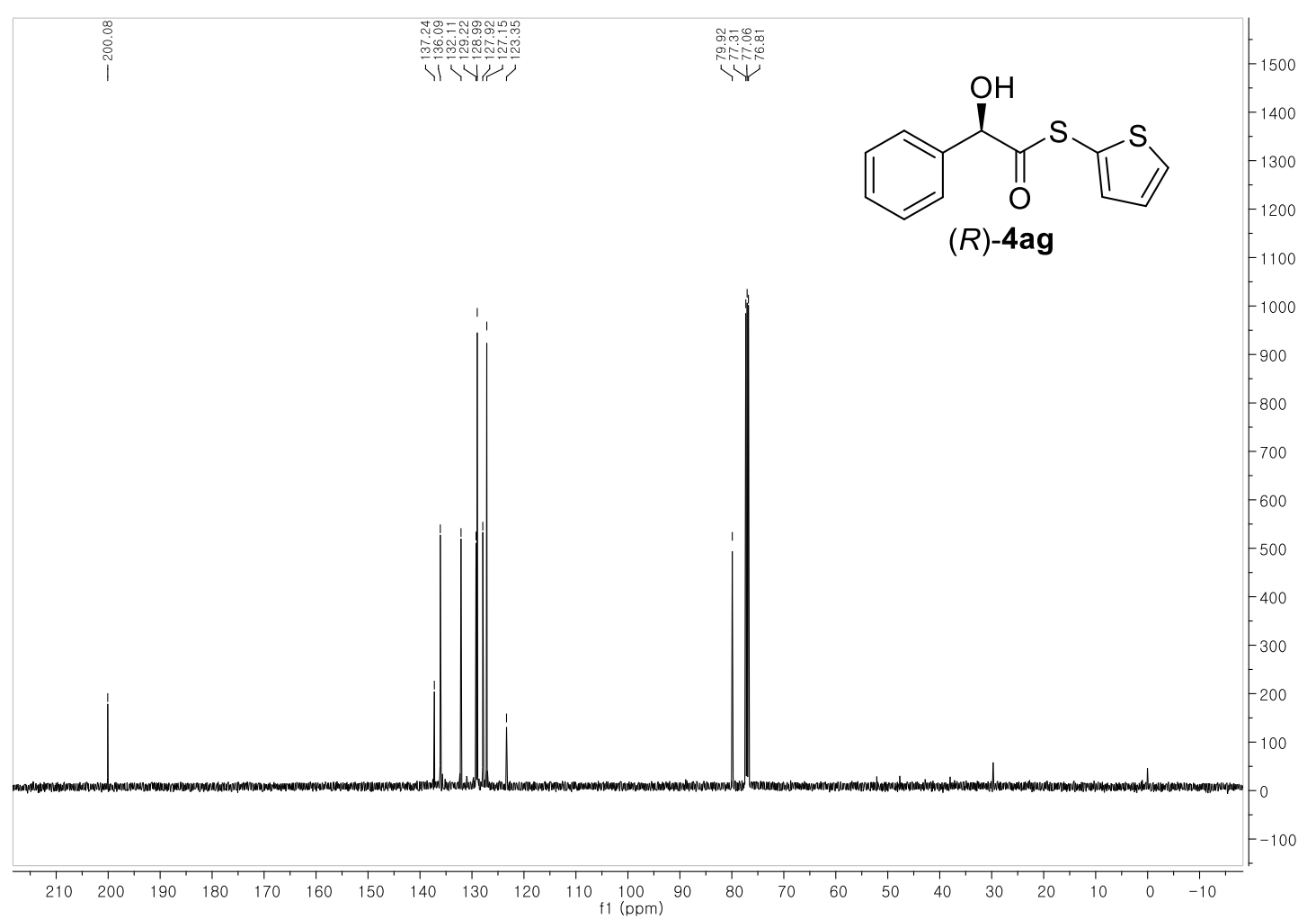

${ }^{13} \mathrm{C}$ NMR spectra of $\mathbf{4 a g}$ 


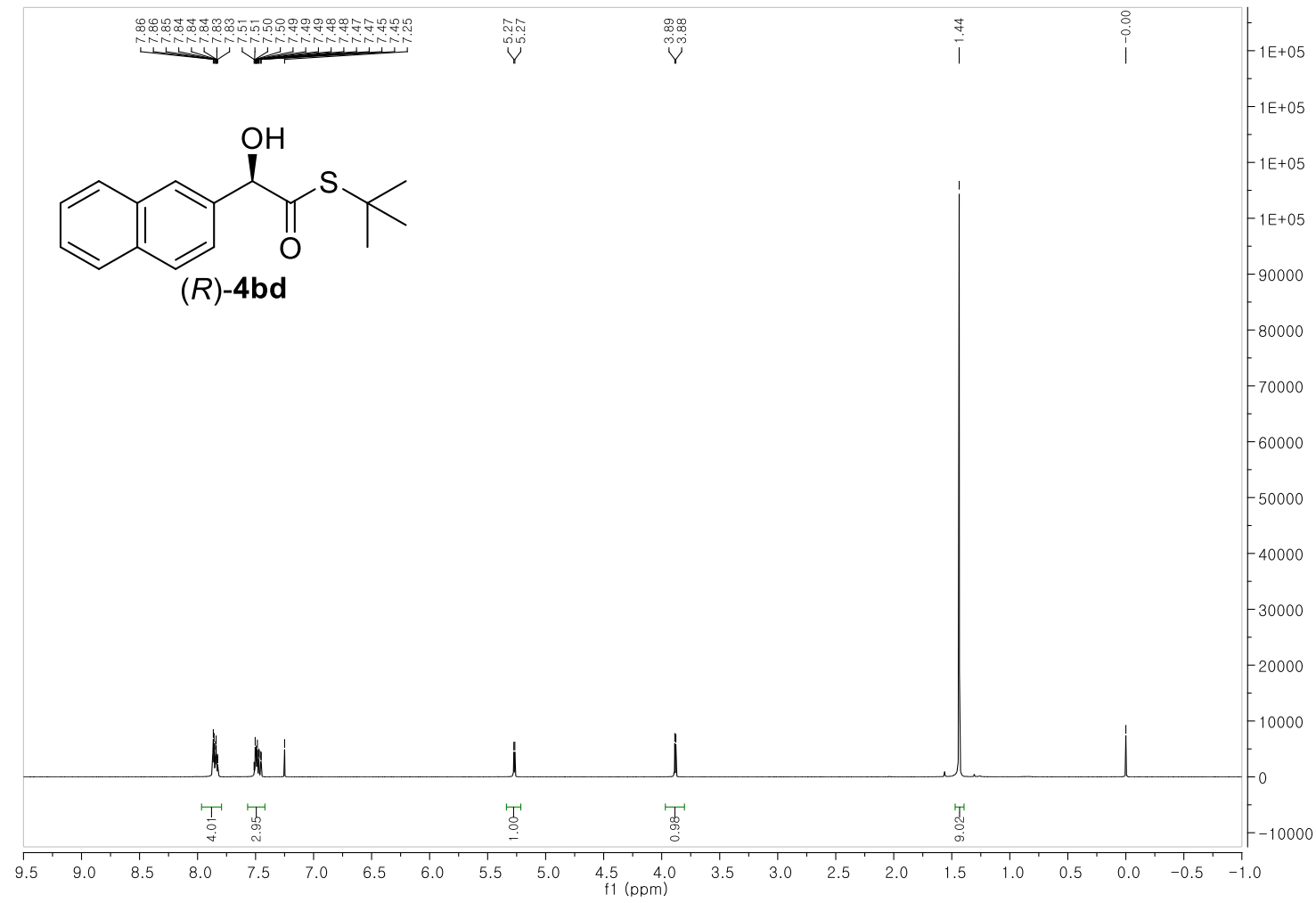

${ }^{1} \mathrm{H}$ NMR spectra of $\mathbf{4 b d}$

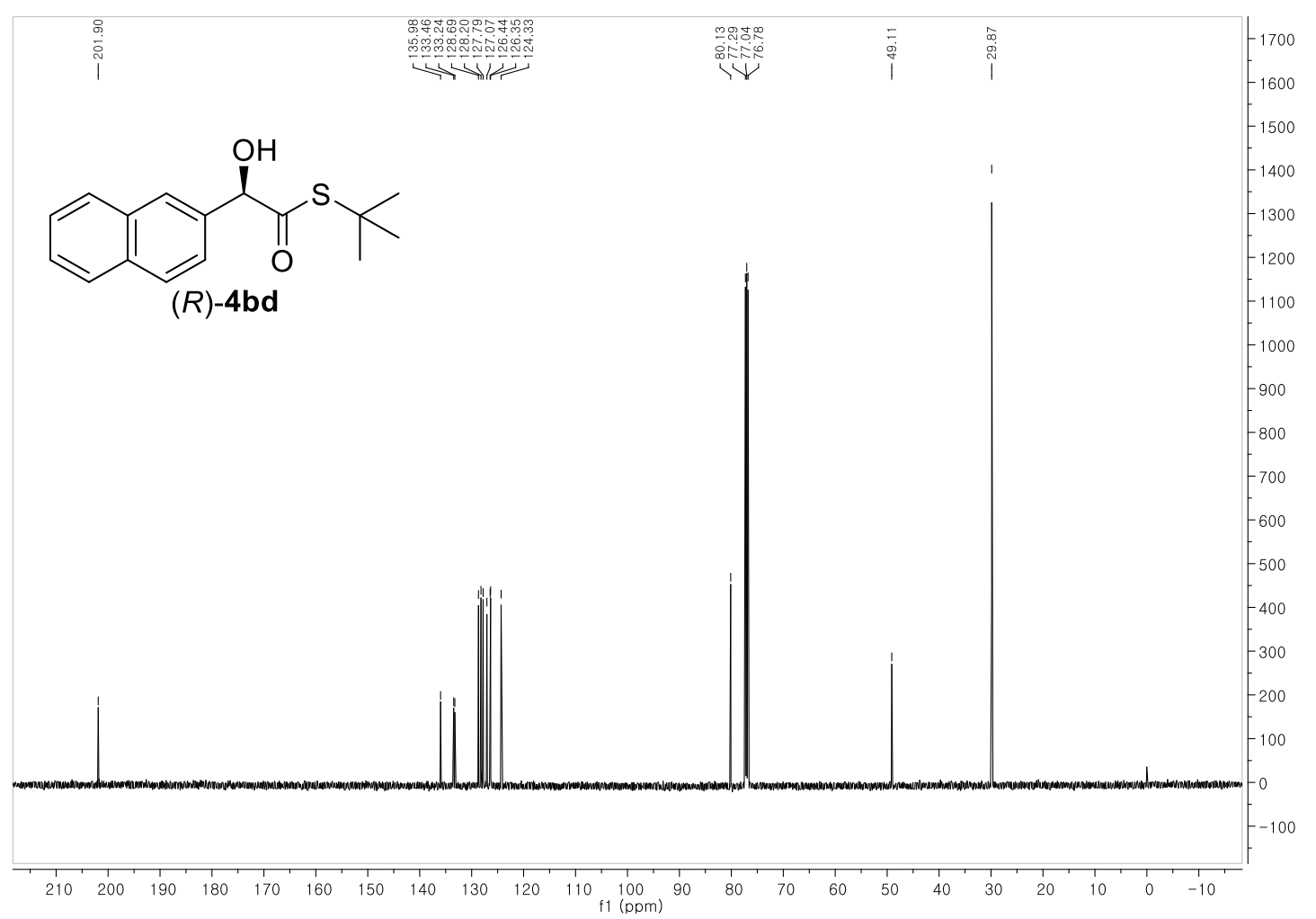

${ }^{13} \mathrm{C}$ NMR spectra of $\mathbf{4 b d}$ 


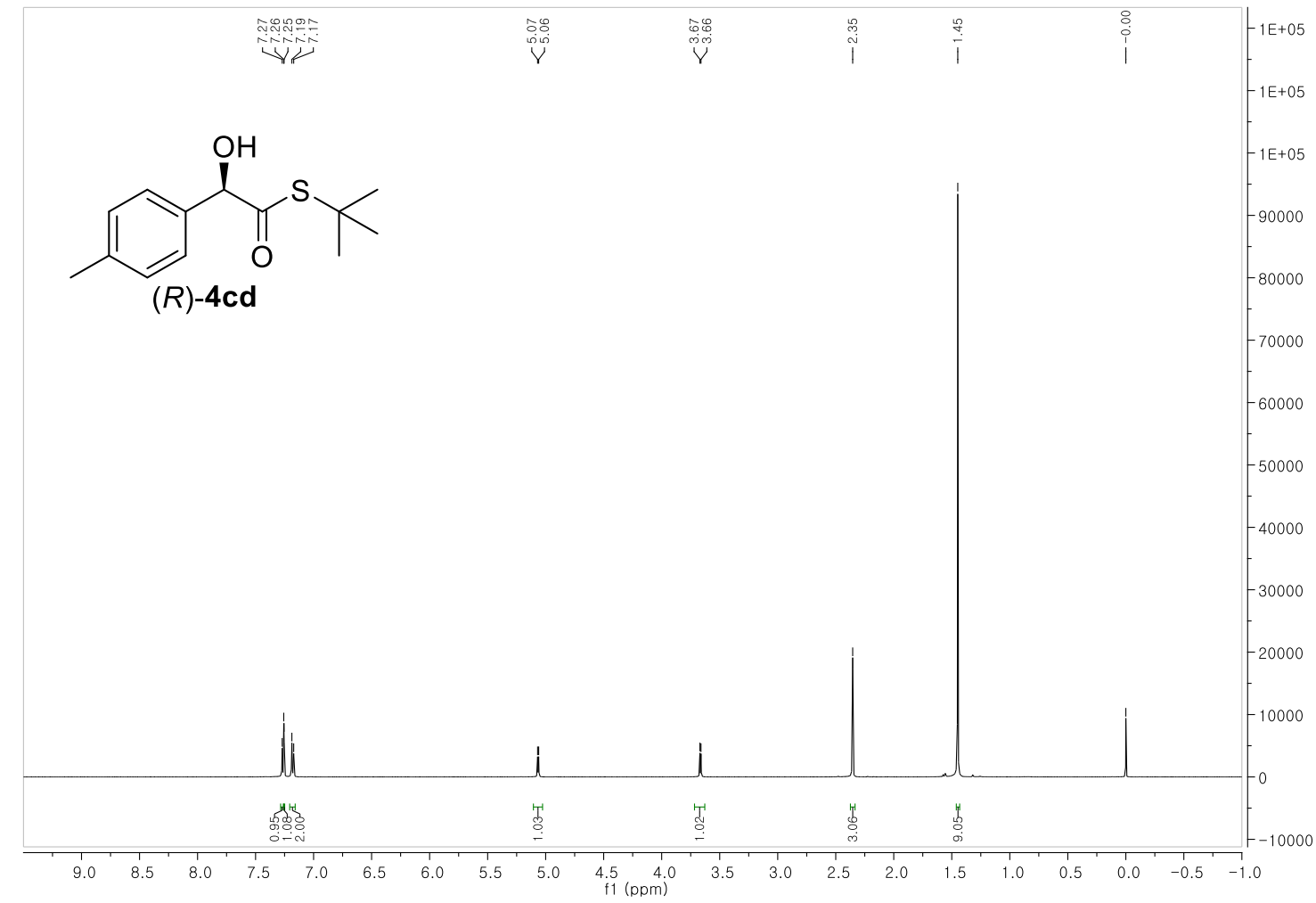

${ }^{1} \mathrm{H}$ NMR spectra of $\mathbf{4 c d}$

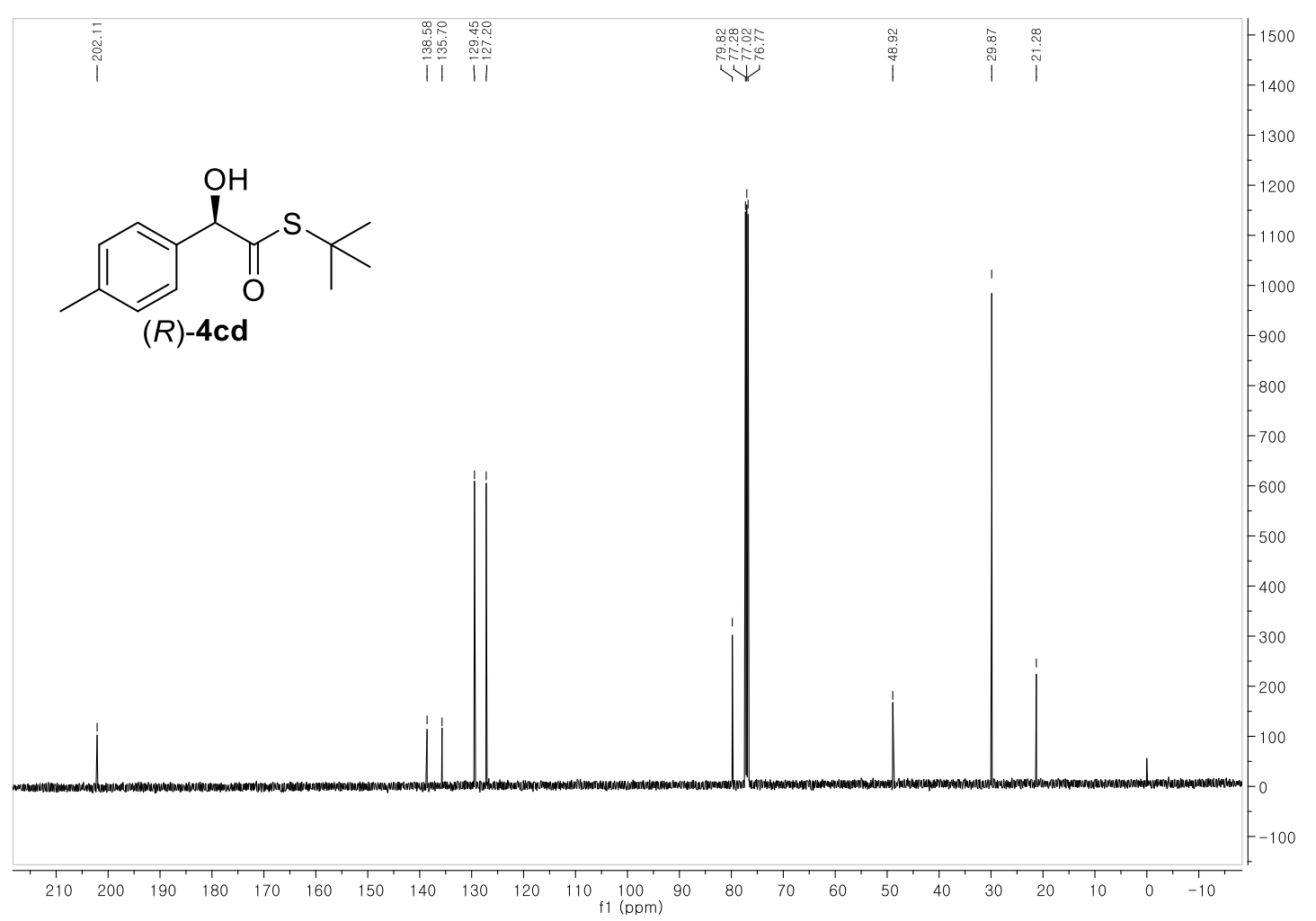

${ }^{13} \mathrm{C}$ NMR spectra of $\mathbf{4 c d}$ 


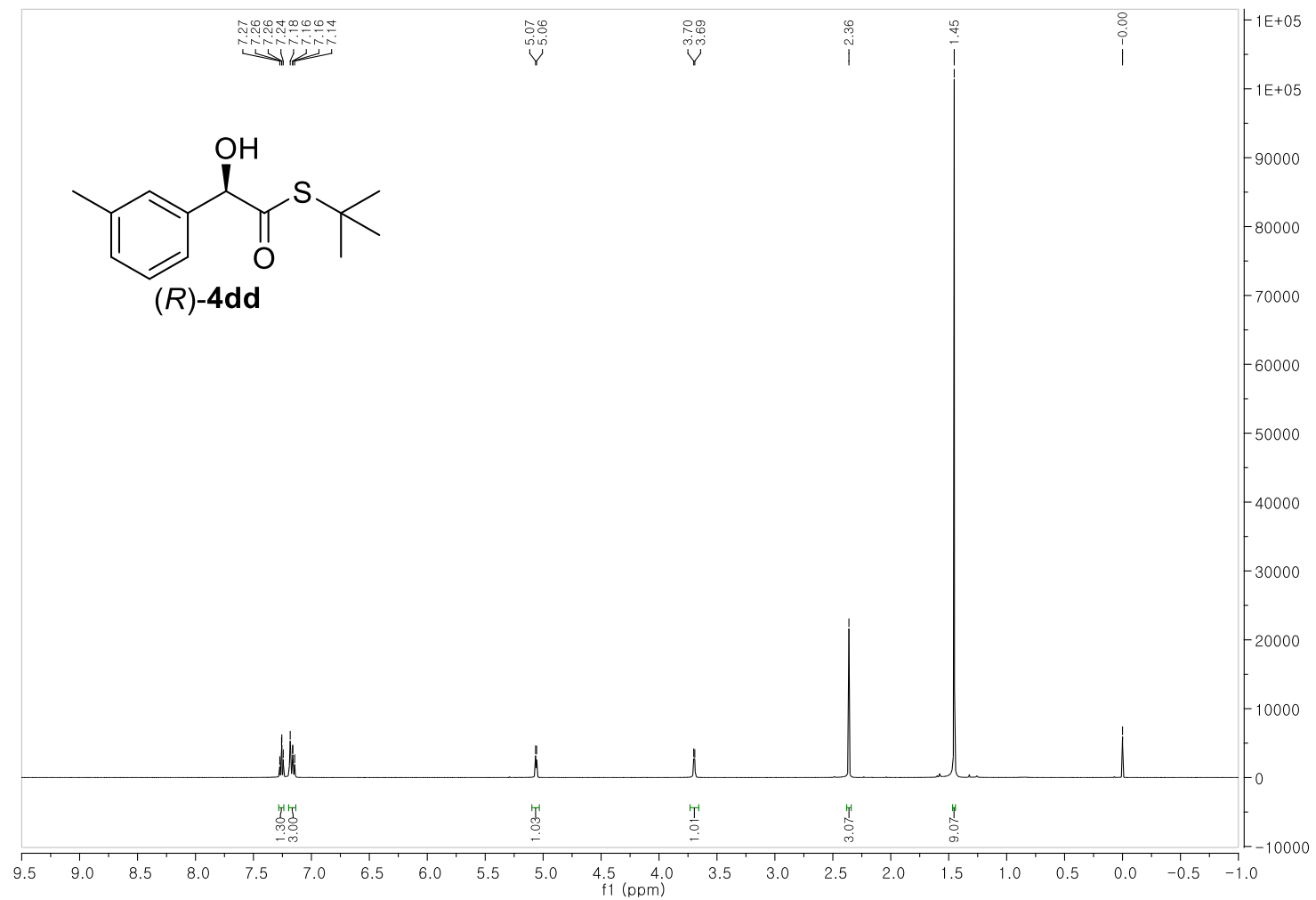

\section{${ }^{1} \mathrm{H}$ NMR spectra of $\mathbf{4 d d}$}

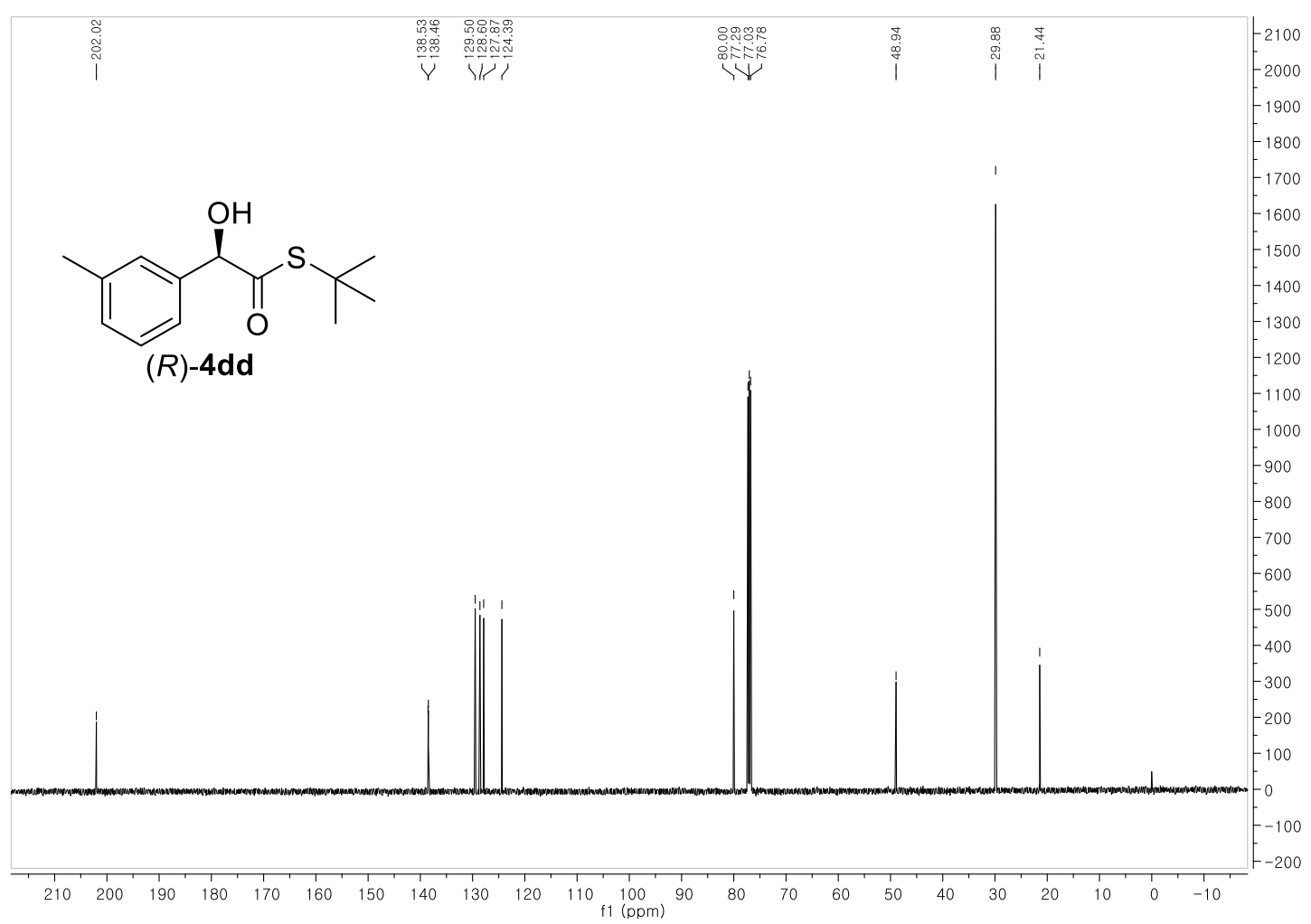

${ }^{13} \mathrm{C}$ NMR spectra of $\mathbf{4 d d}$ 


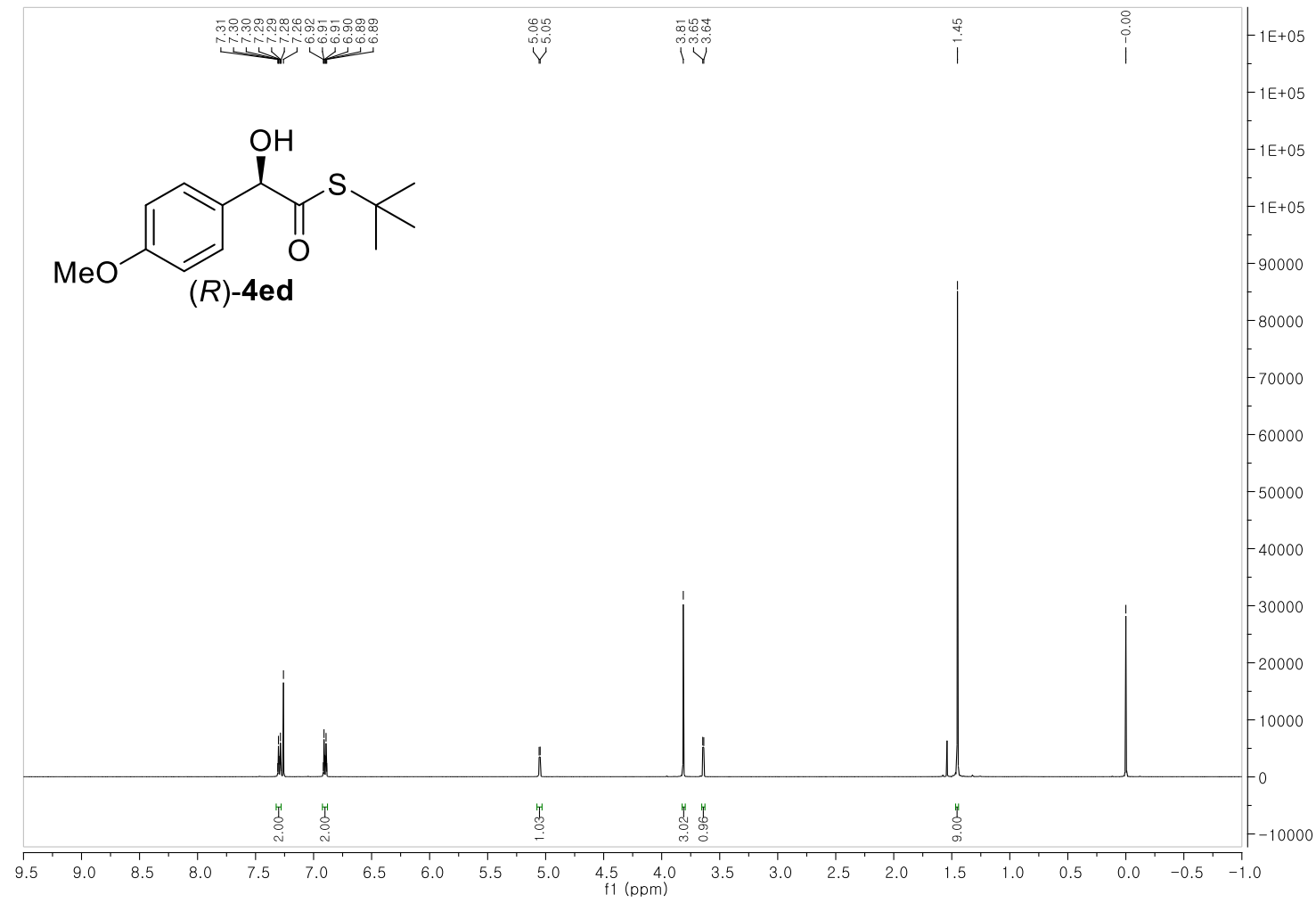

\section{${ }^{1} \mathrm{H}$ NMR spectra of $\mathbf{4 e d}$}

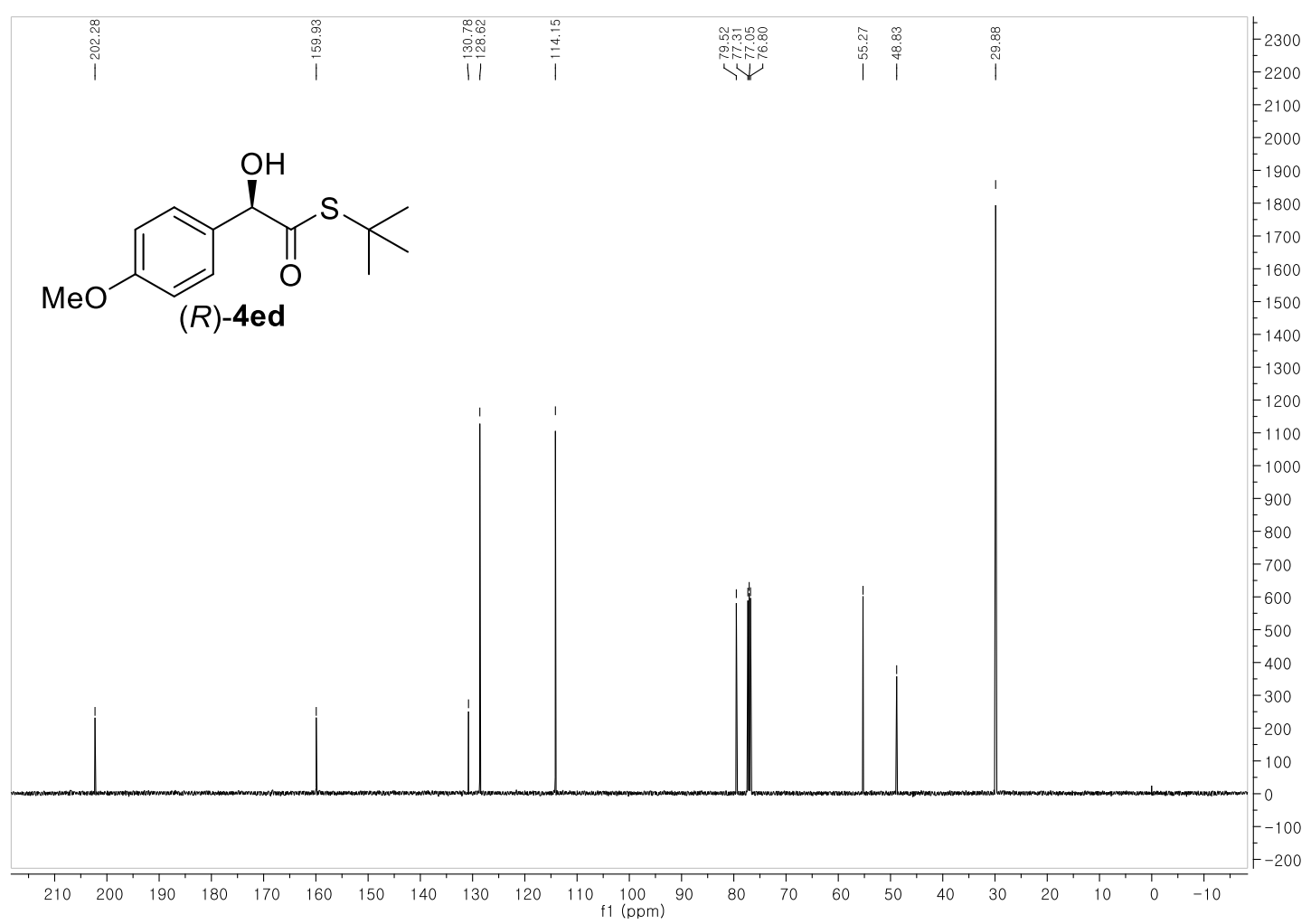

${ }^{13} \mathrm{C}$ NMR spectra of $\mathbf{4 e d}$ 


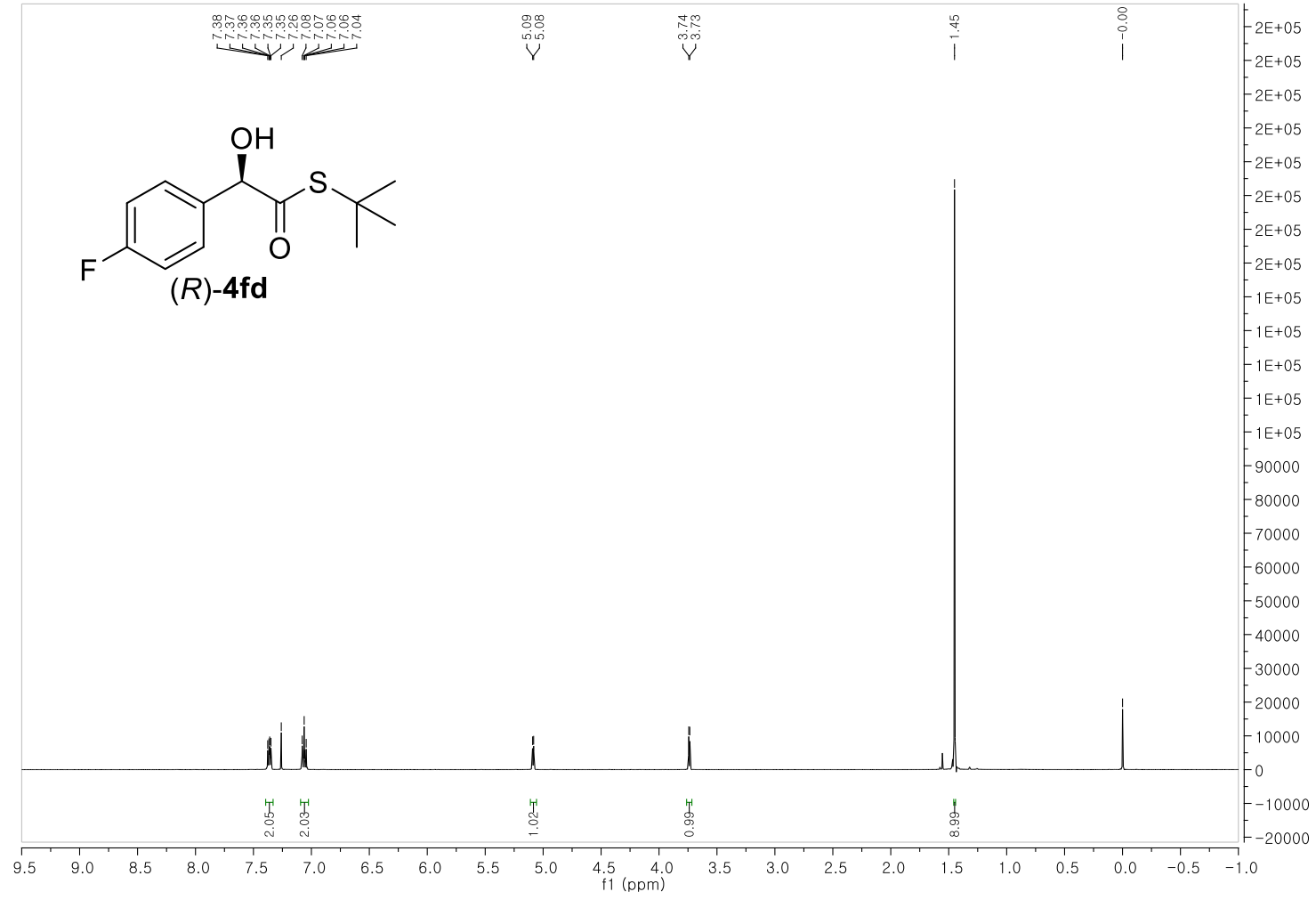

${ }^{1} \mathrm{H}$ NMR spectra of $\mathbf{4 f d}$

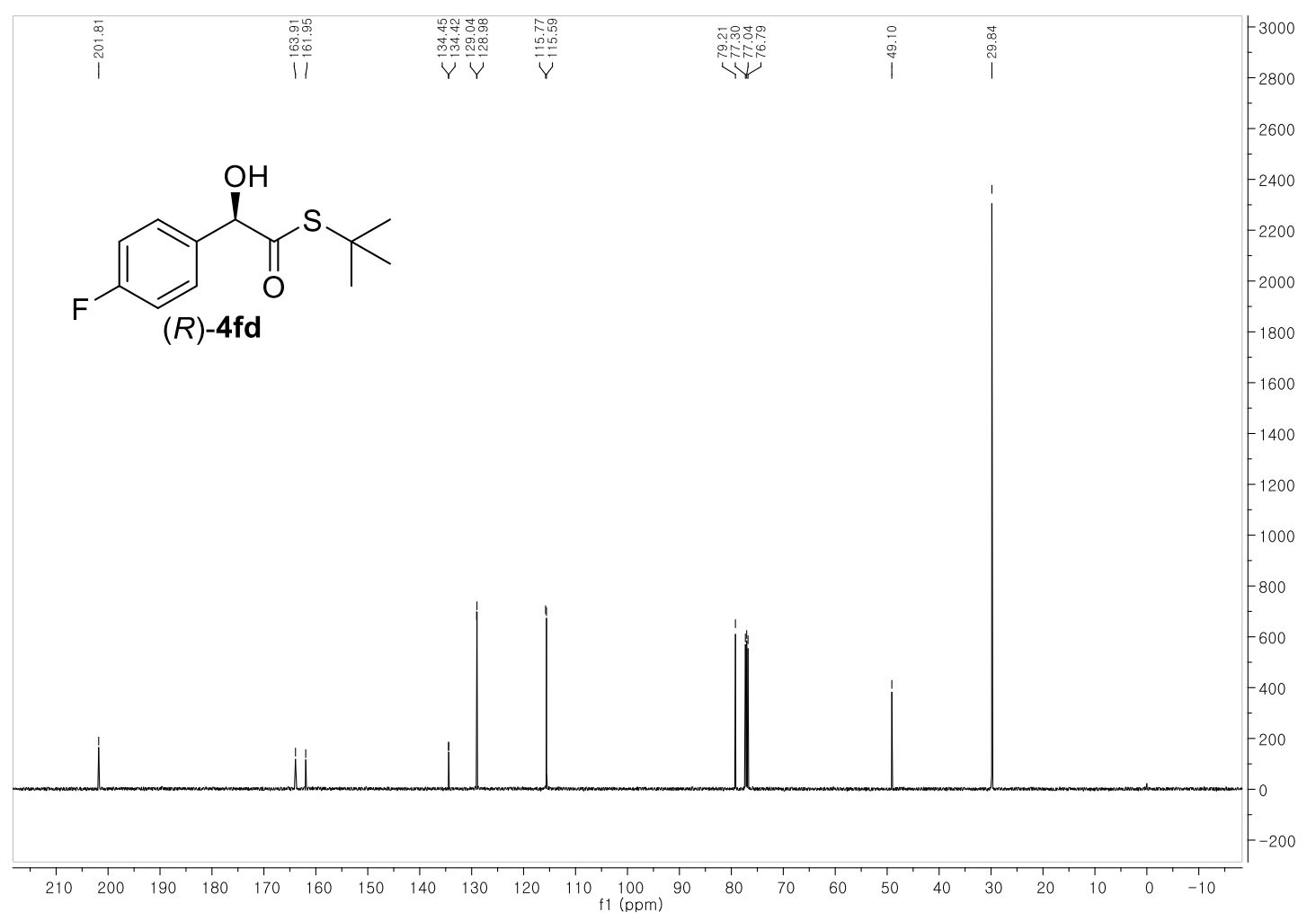

${ }^{13} \mathrm{C}$ NMR spectra of $\mathbf{4 f d}$ 


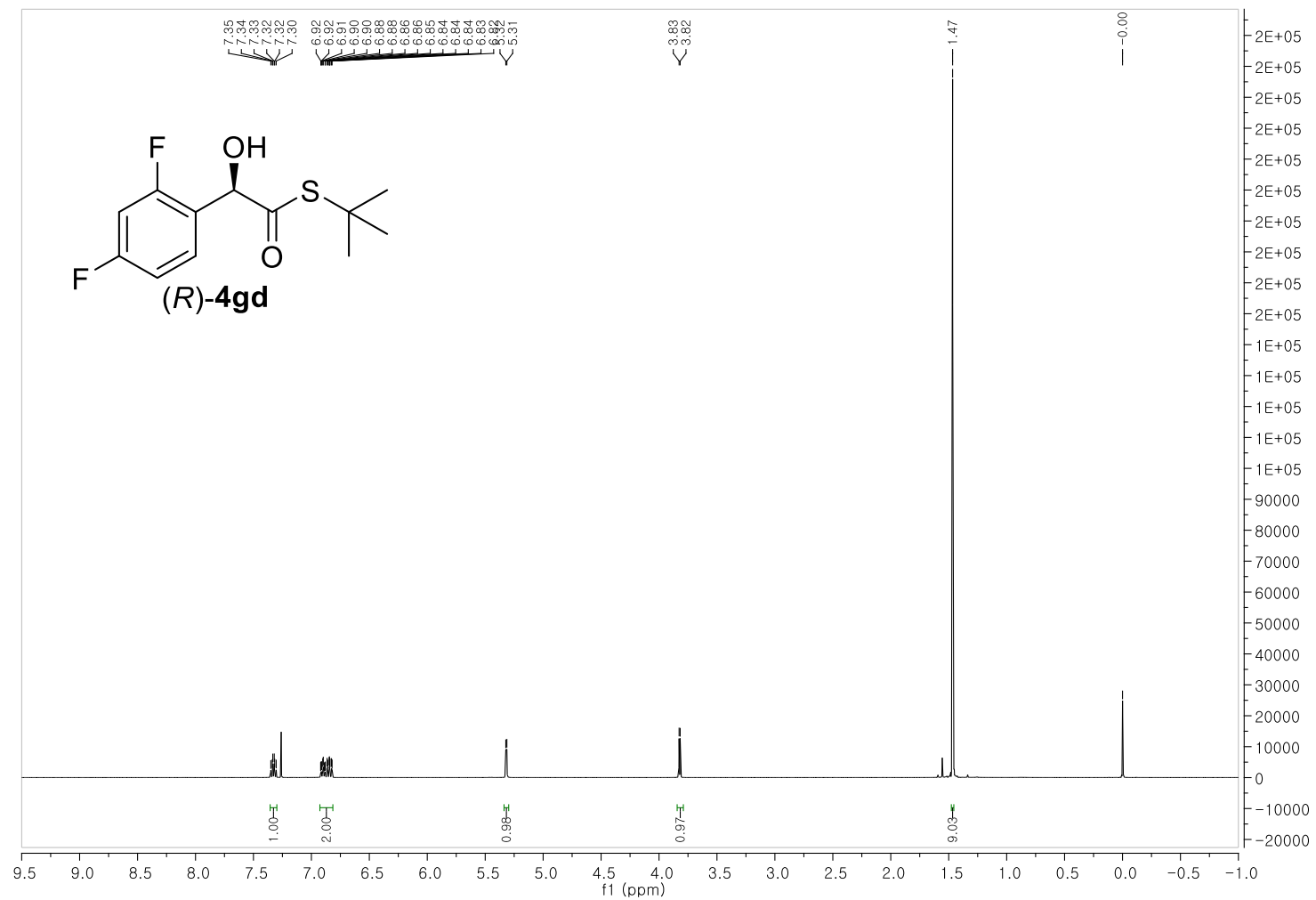

\section{${ }^{1} \mathrm{H}$ NMR spectra of $\mathbf{4 g d}$}

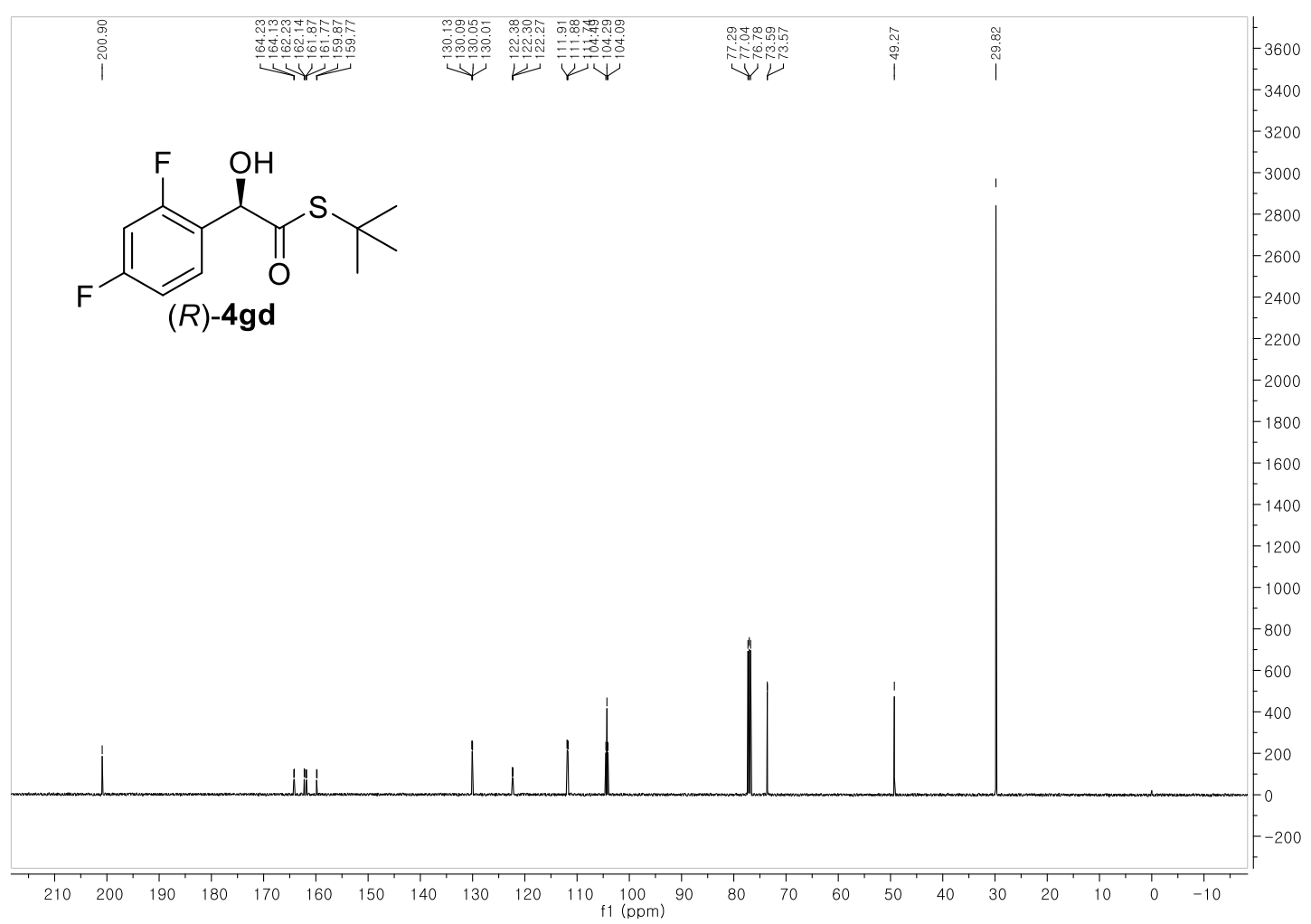

${ }^{13} \mathrm{C}$ NMR spectra of $\mathbf{4 g d}$ 


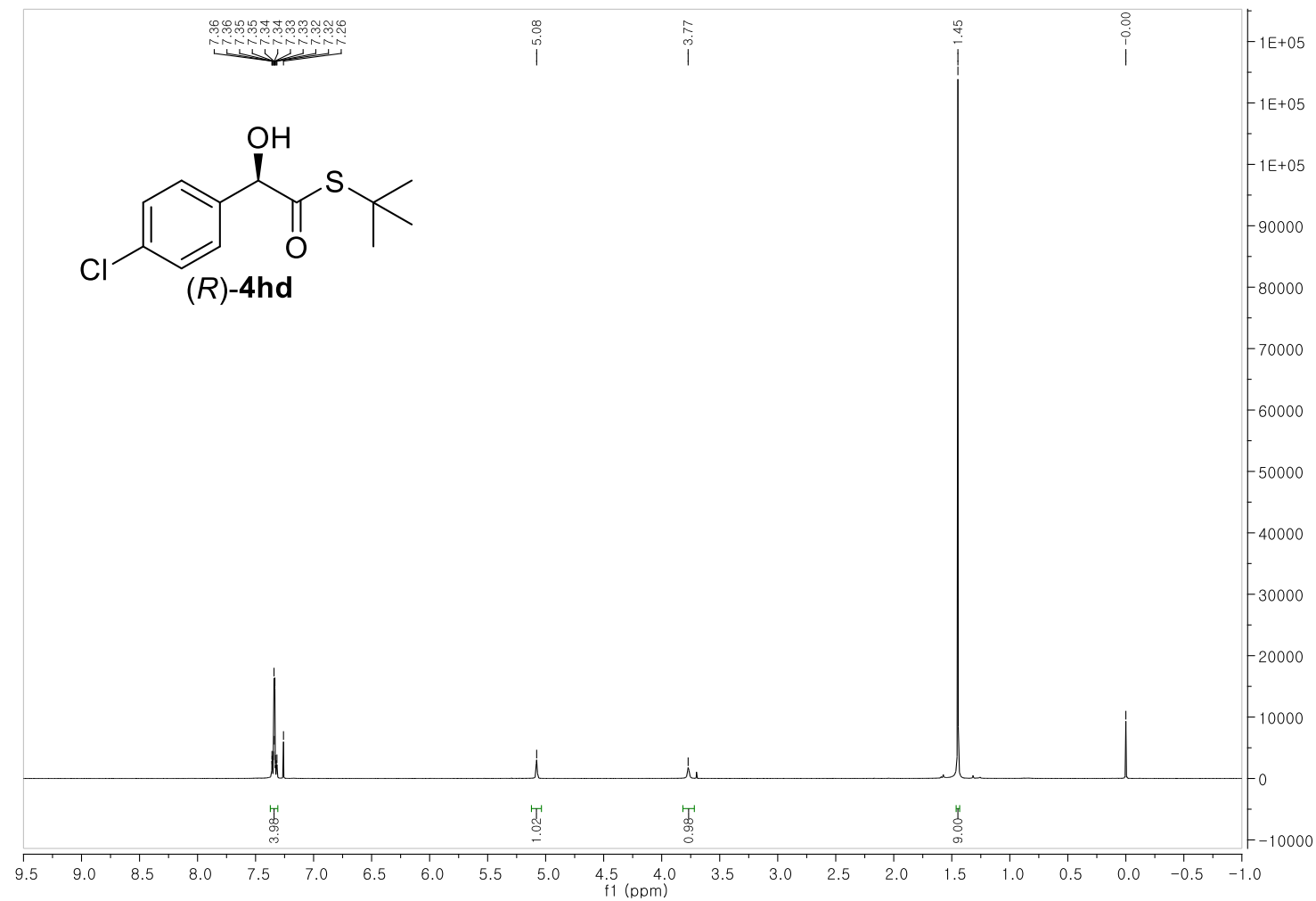

${ }^{1} \mathrm{H}$ NMR spectra of $\mathbf{4 h d}$

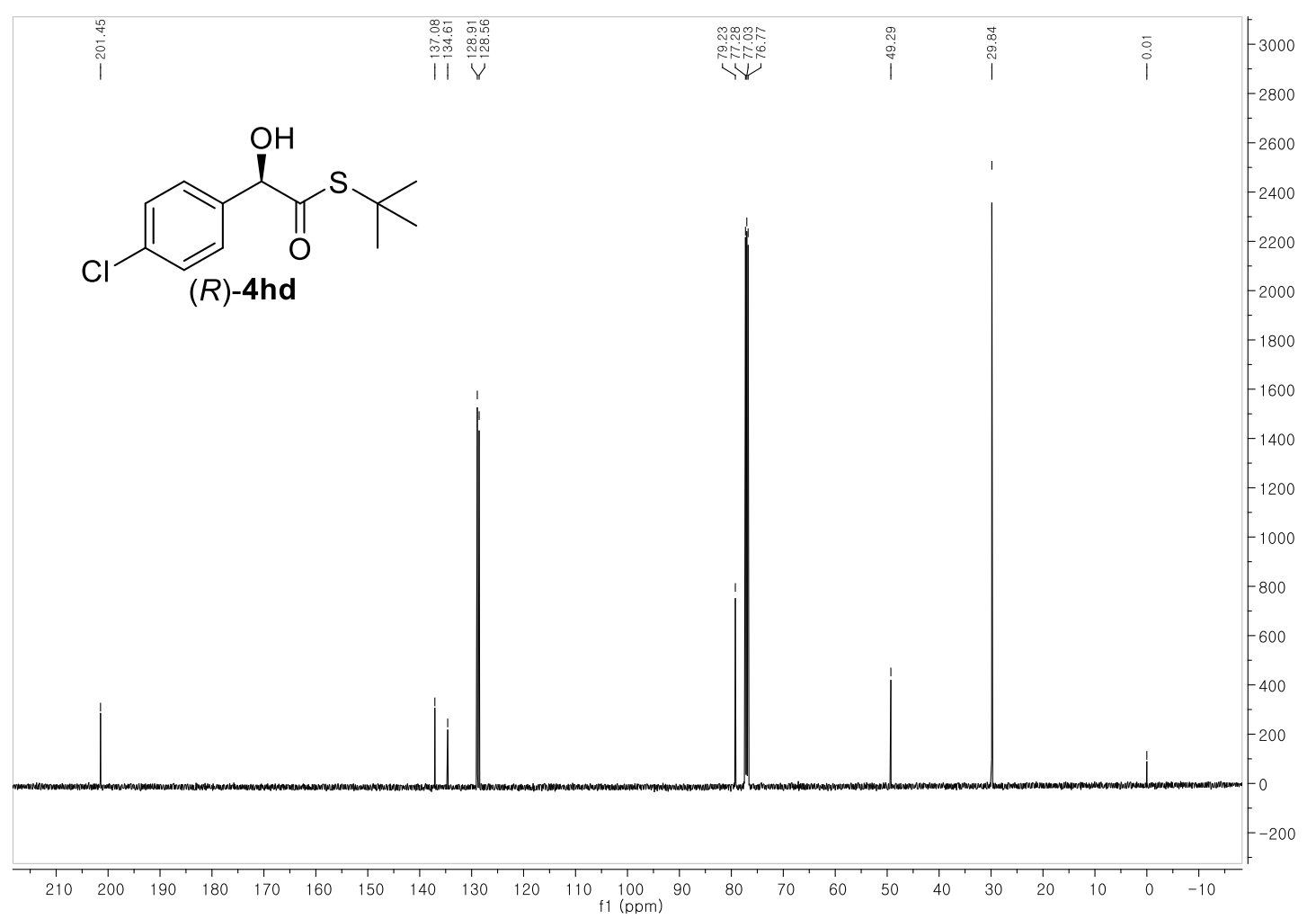

${ }^{13} \mathrm{C}$ NMR spectra of $\mathbf{4 h d}$ 


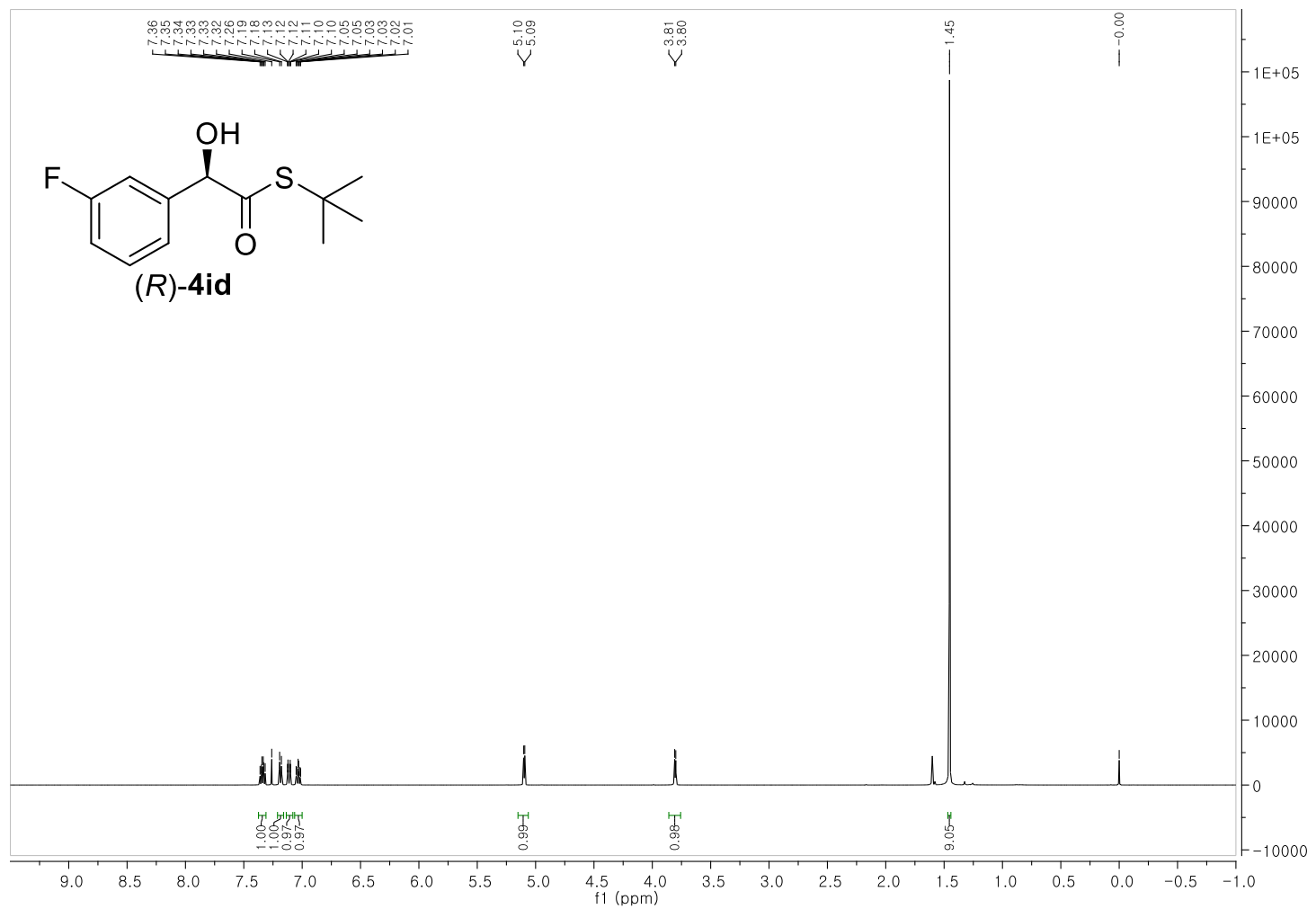

${ }^{1} \mathrm{H}$ NMR spectra of $\mathbf{4 i d}$

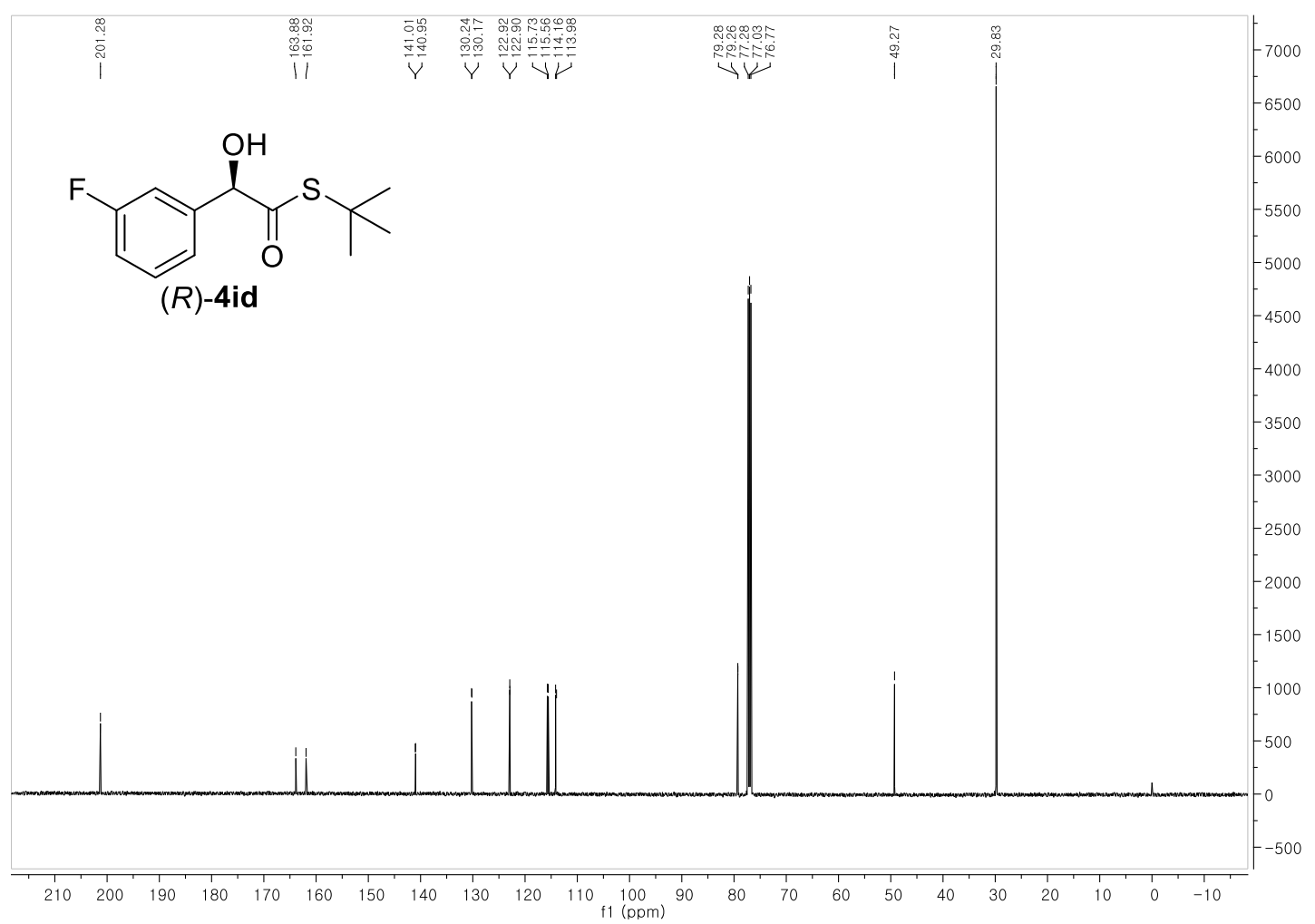

${ }^{13} \mathrm{C}$ NMR spectra of $\mathbf{4 i d}$ 


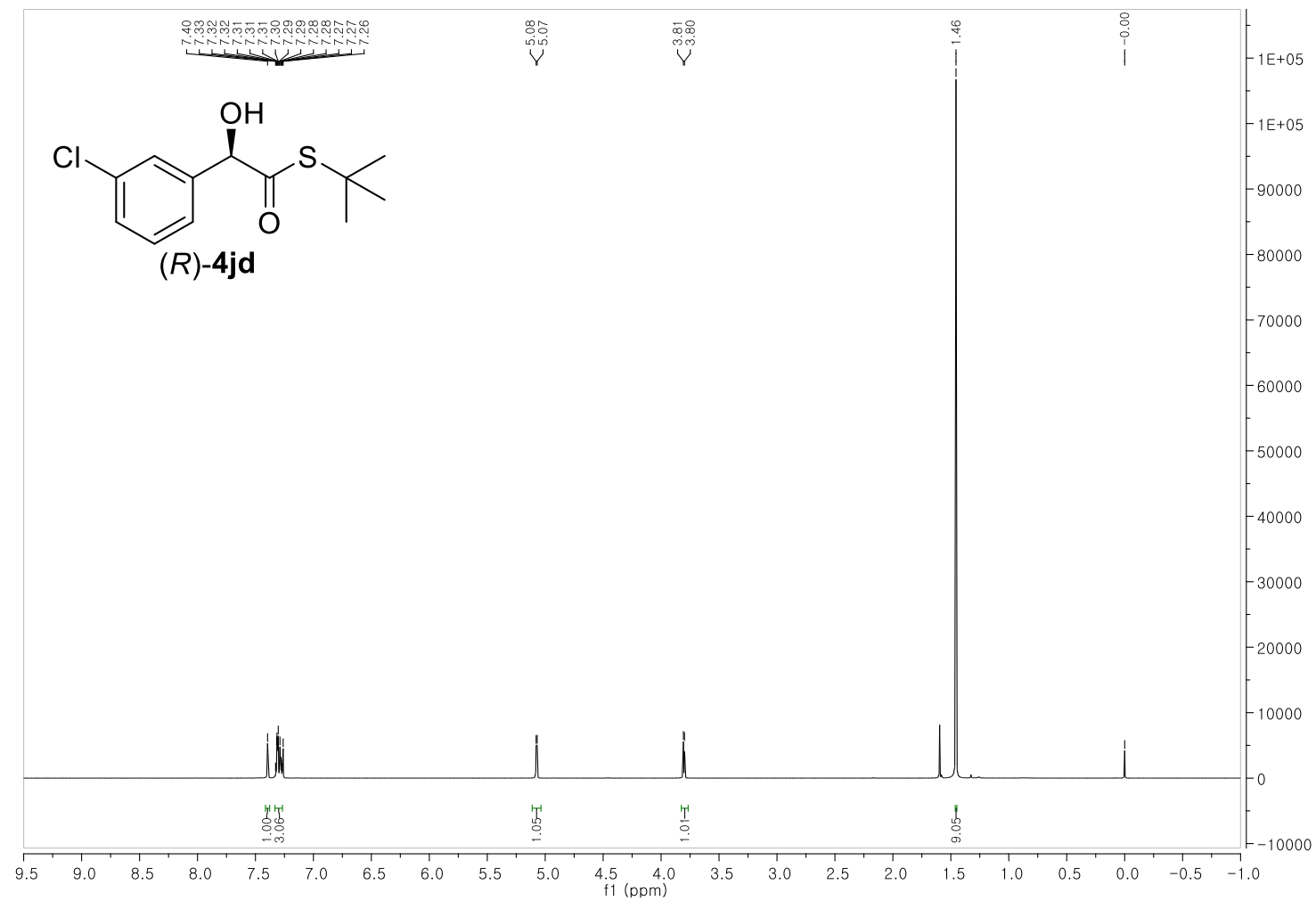

${ }^{1} \mathrm{H}$ NMR spectra of $\mathbf{4 j d}$

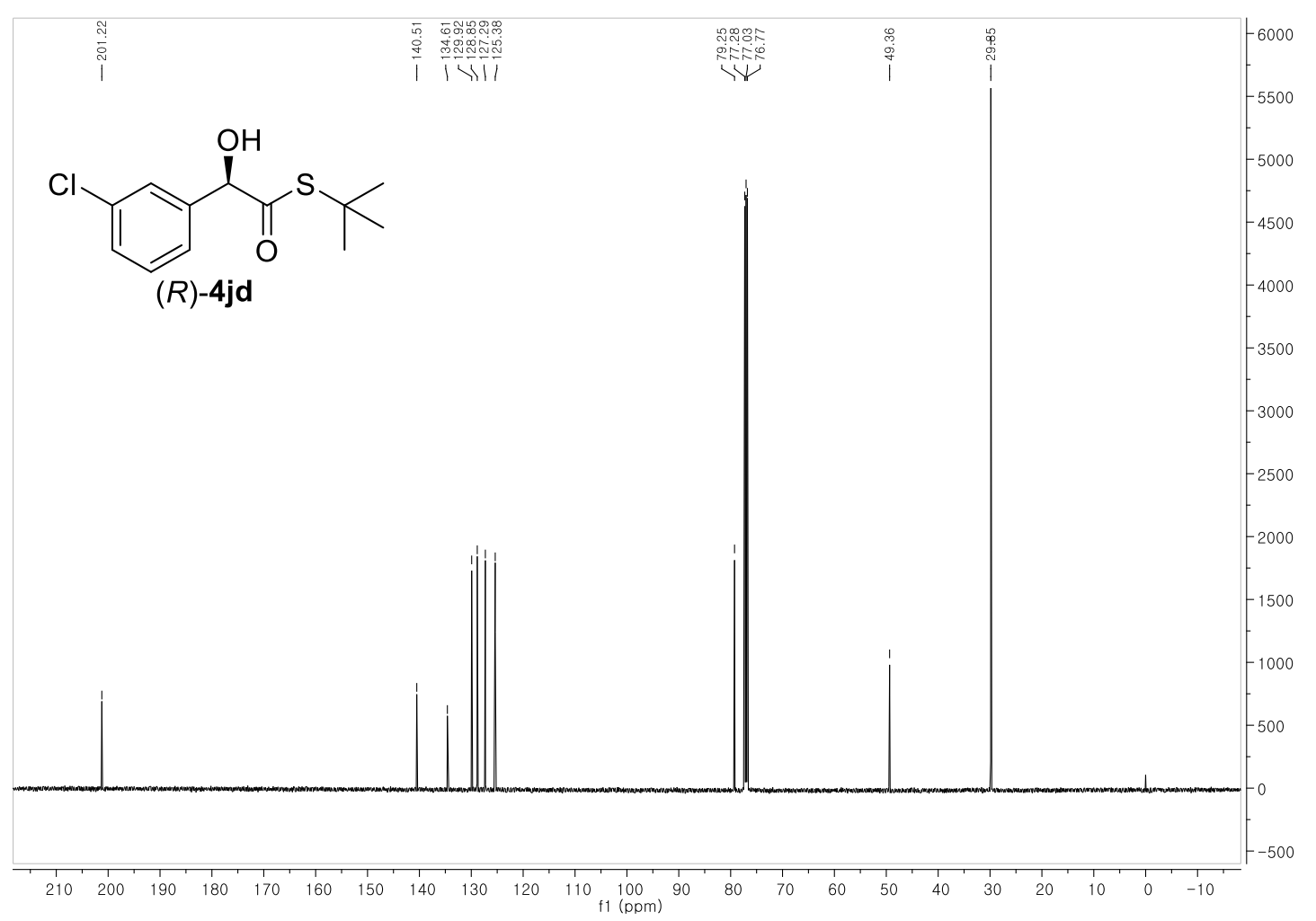

${ }^{13} \mathrm{C}$ NMR spectra of $\mathbf{4 j d}$ 


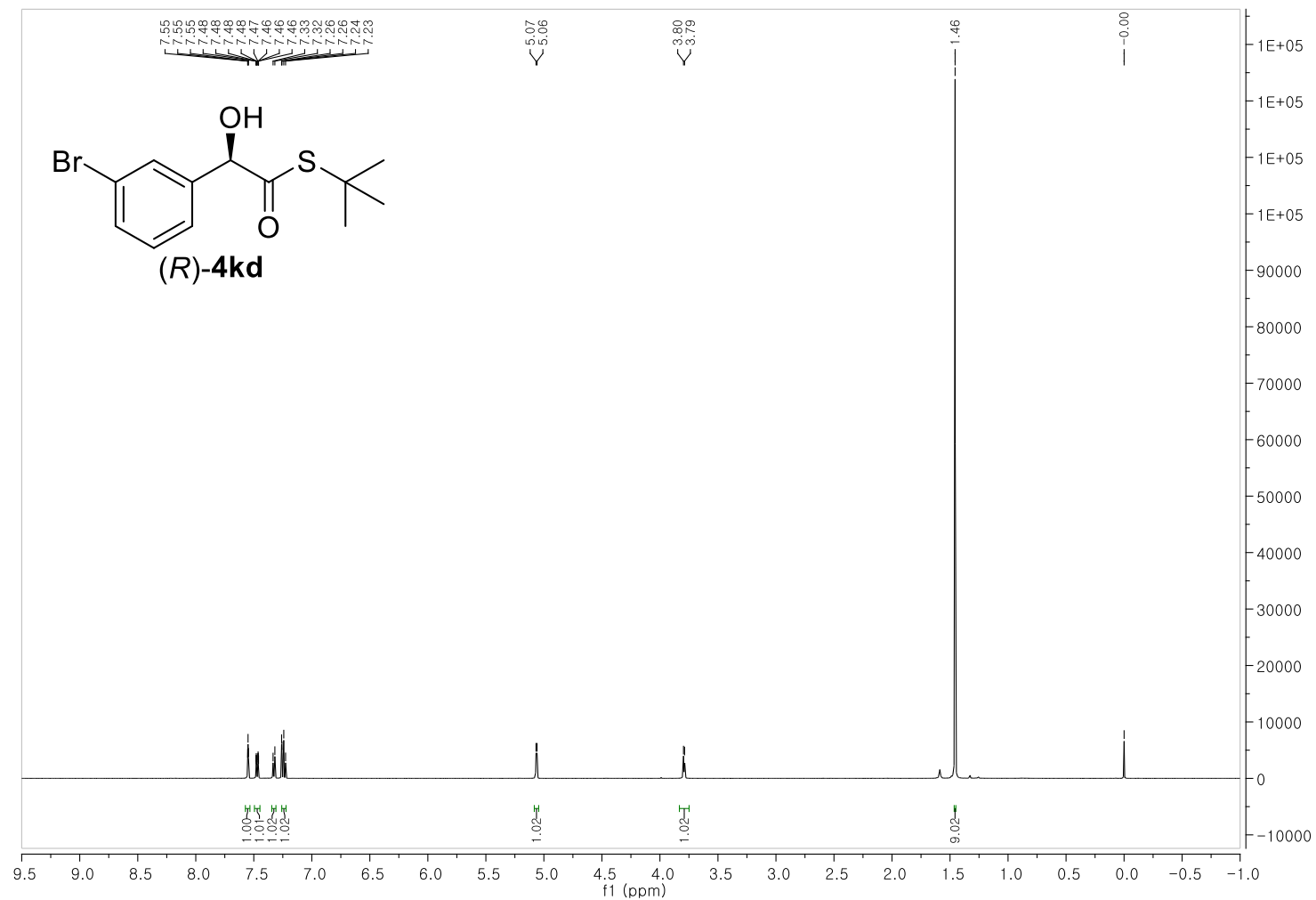

${ }^{1} \mathrm{H}$ NMR spectra of $\mathbf{4 k d}$

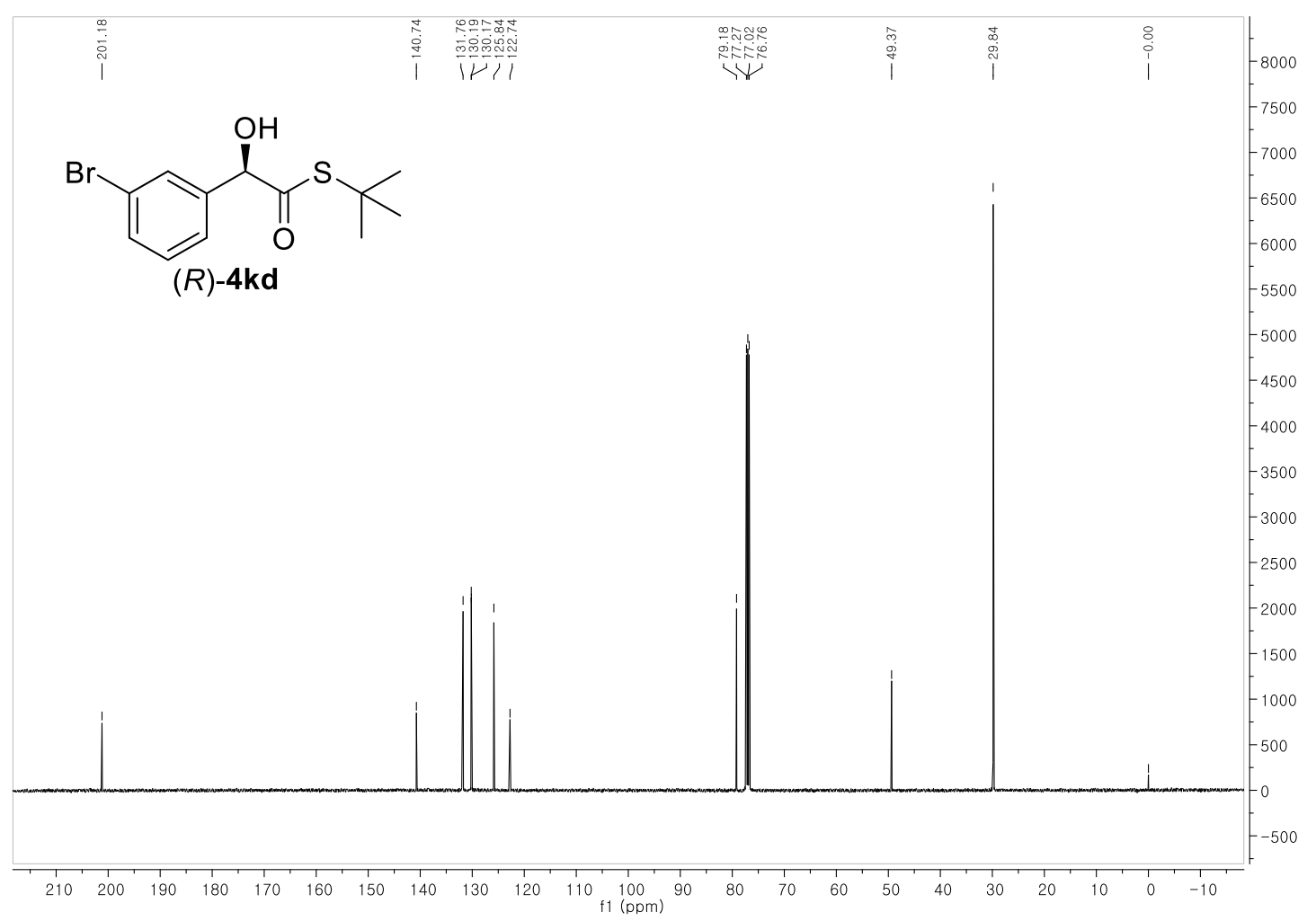

${ }^{13} \mathrm{C}$ NMR spectra of $\mathbf{4 k d}$ 


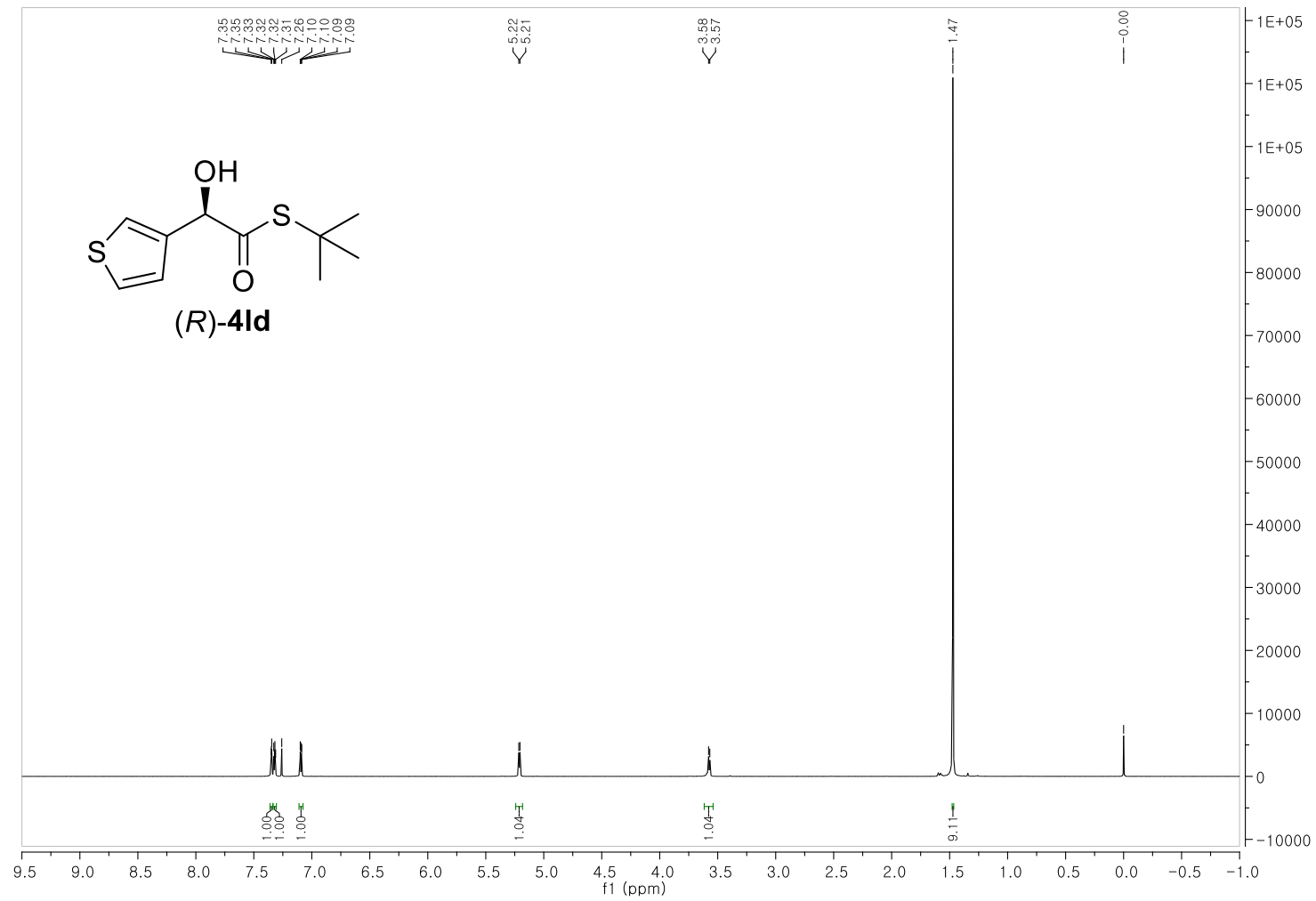

\section{${ }^{1} \mathrm{H}$ NMR spectra of 4ld}

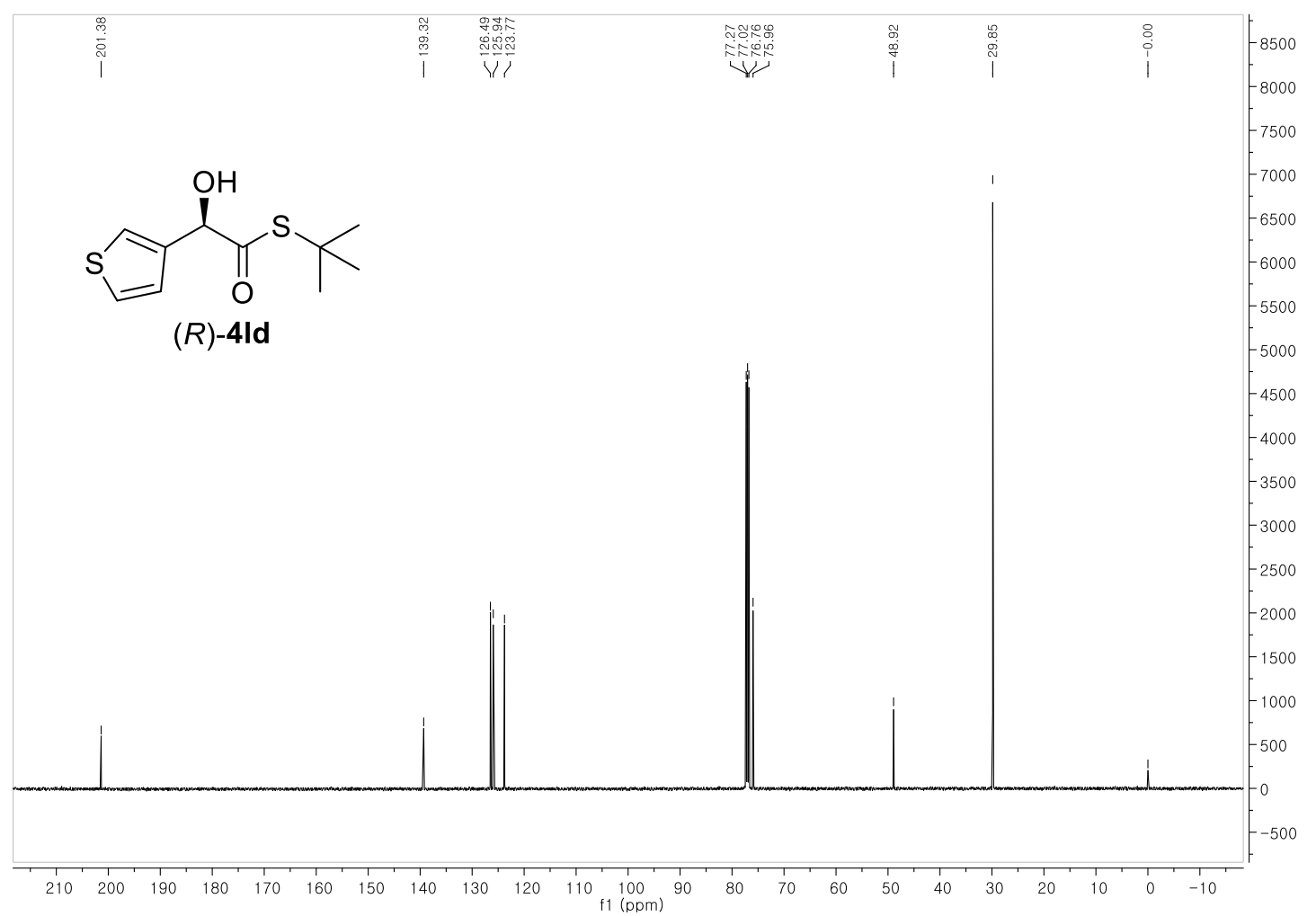

${ }^{13} \mathrm{C}$ NMR spectra of 4ld 


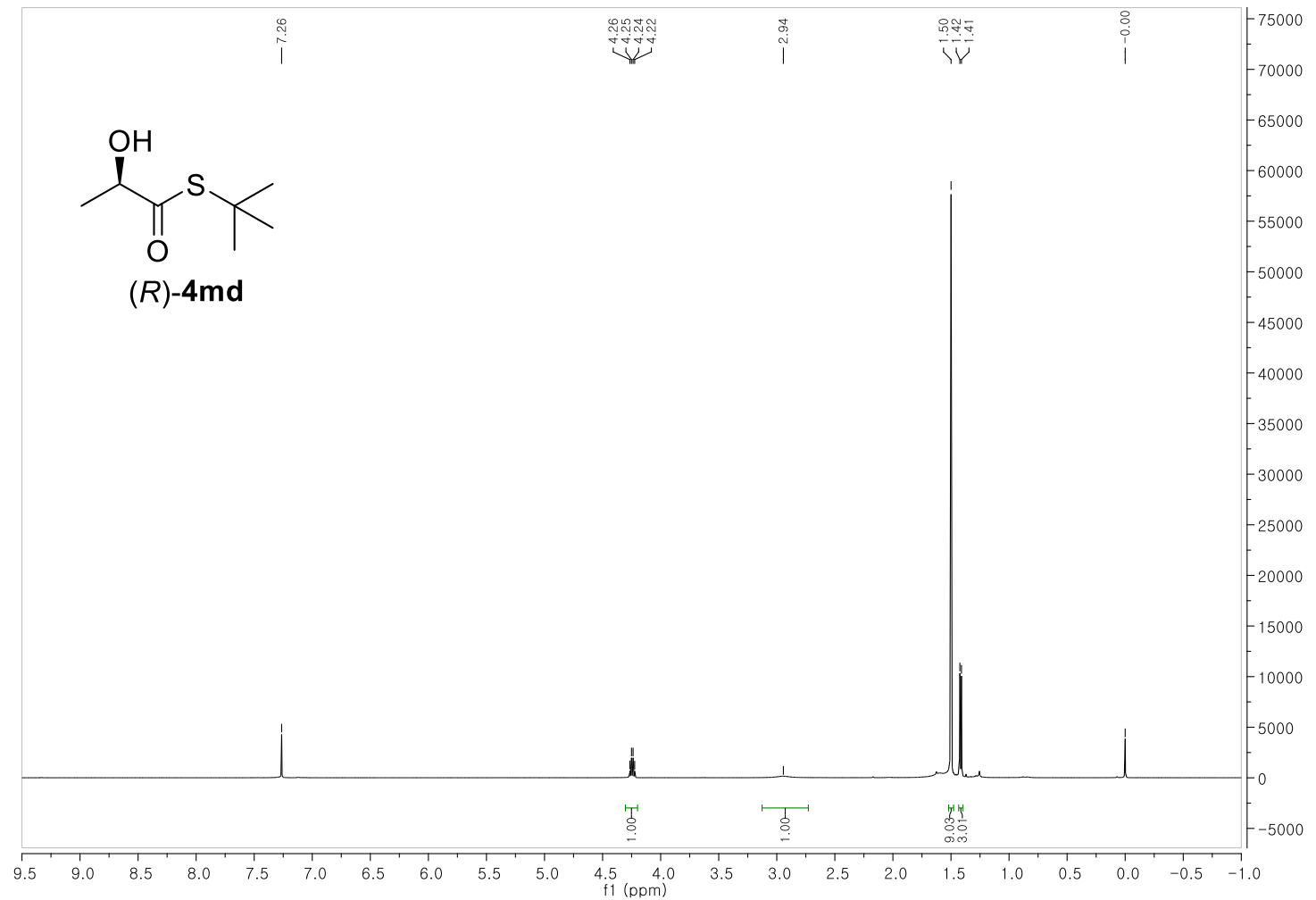

${ }^{1} \mathrm{H}$ NMR spectra of $\mathbf{4 m d}$

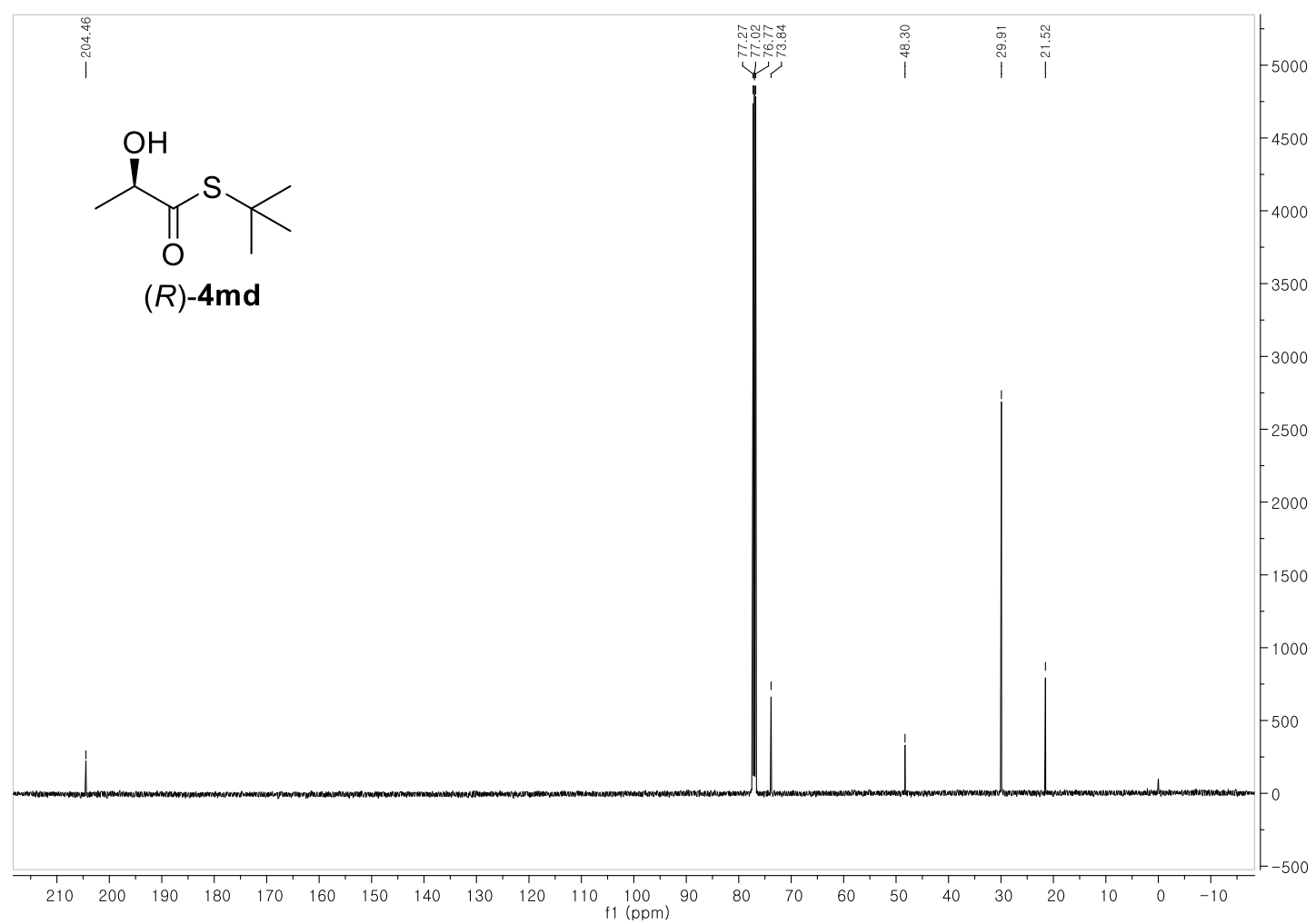

${ }^{13} \mathrm{C}$ NMR spectra of $\mathbf{4 m d}$ 


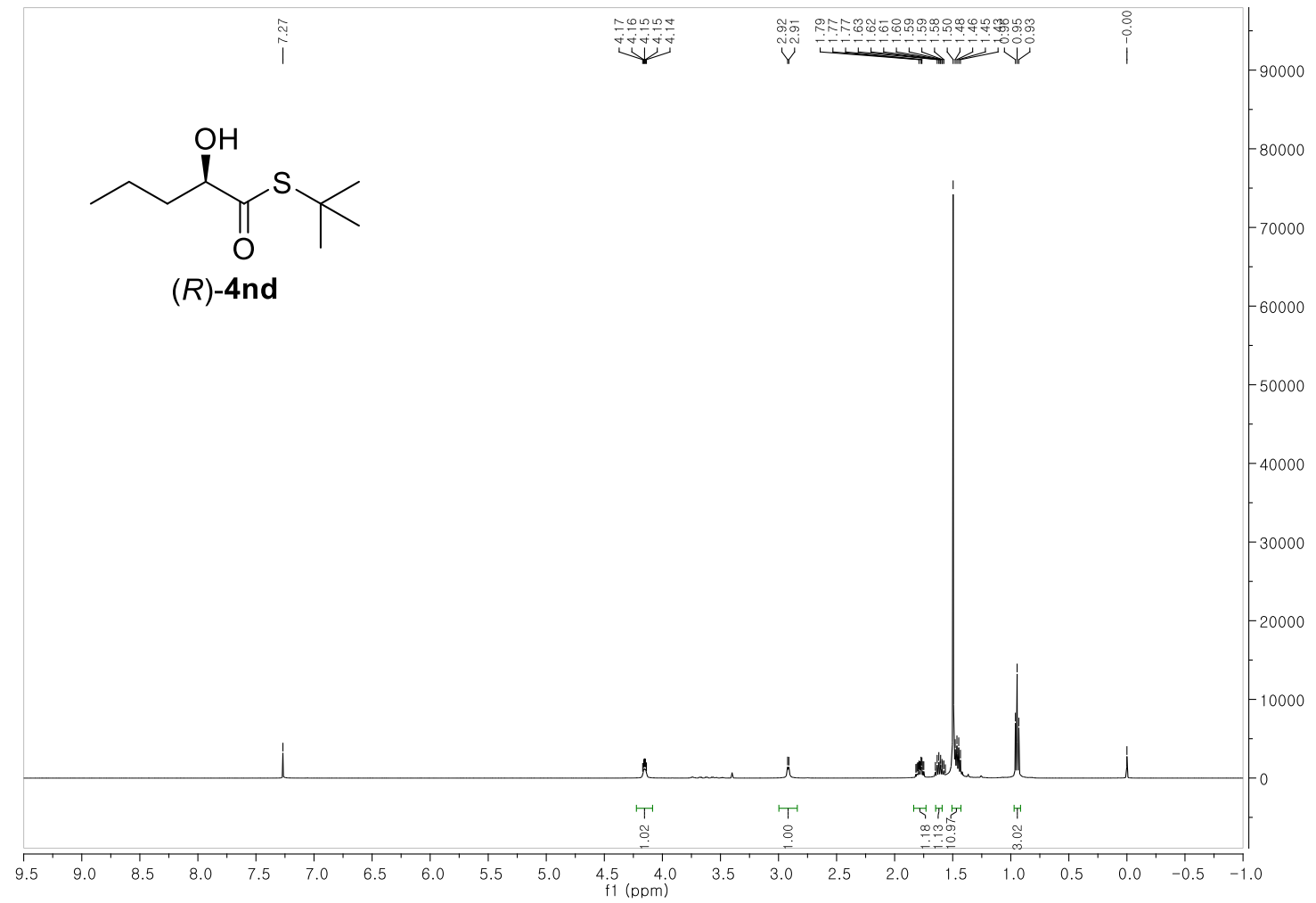

\section{${ }^{1} \mathrm{H}$ NMR spectra of 4 nd}

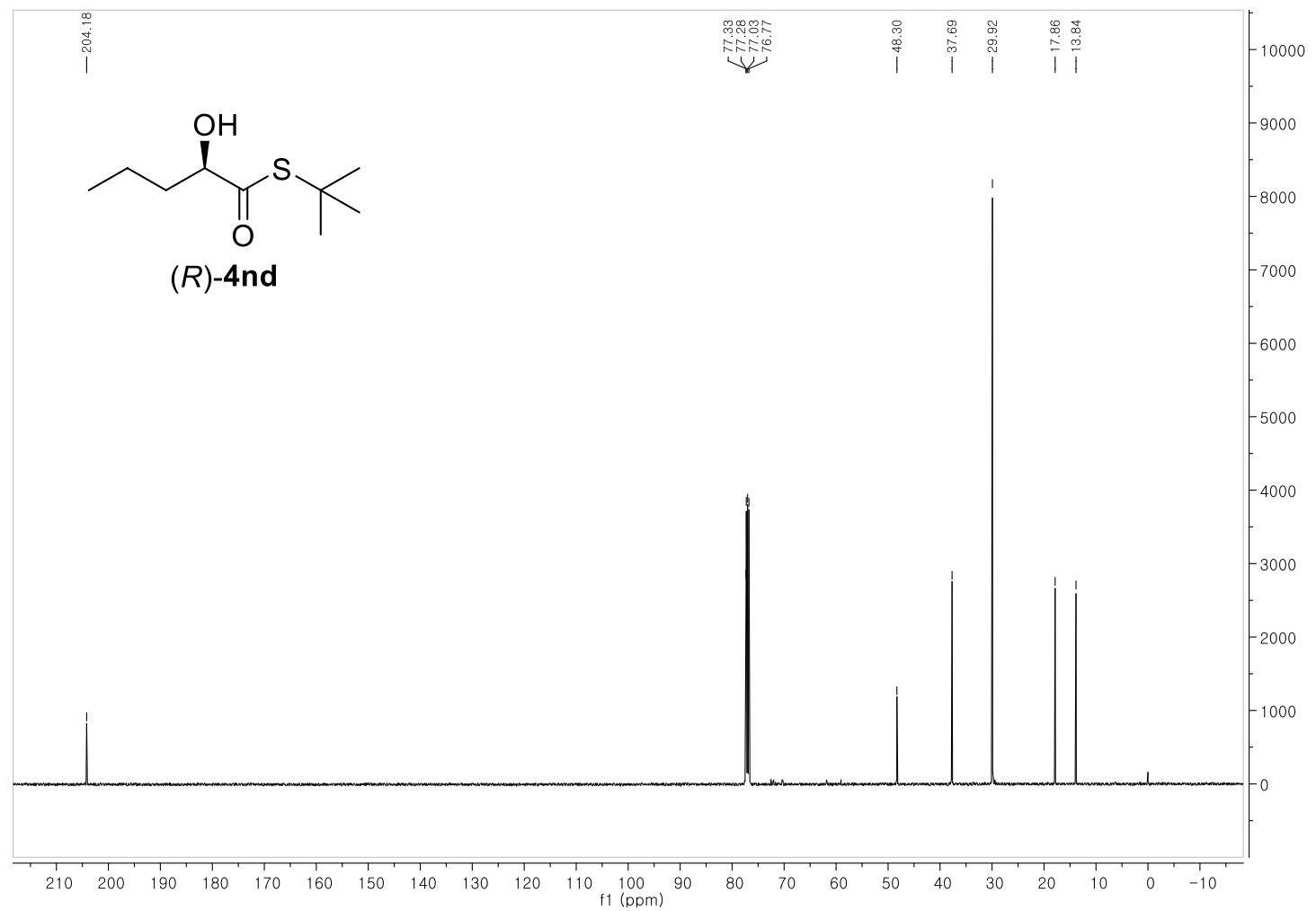

${ }^{13} \mathrm{C}$ NMR spectra of 4 nd 


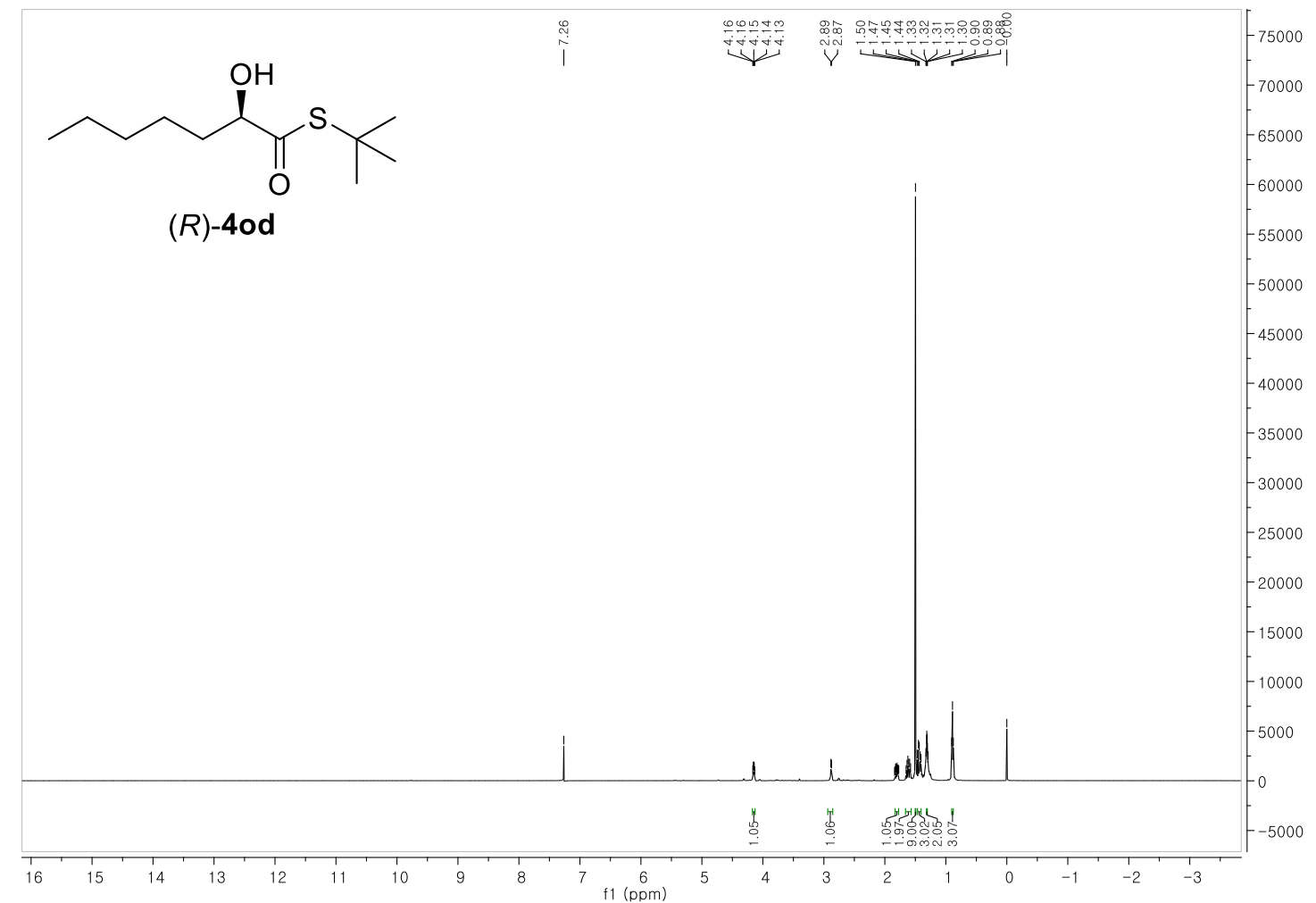

${ }^{1} \mathrm{H}$ NMR spectra of $40 d$

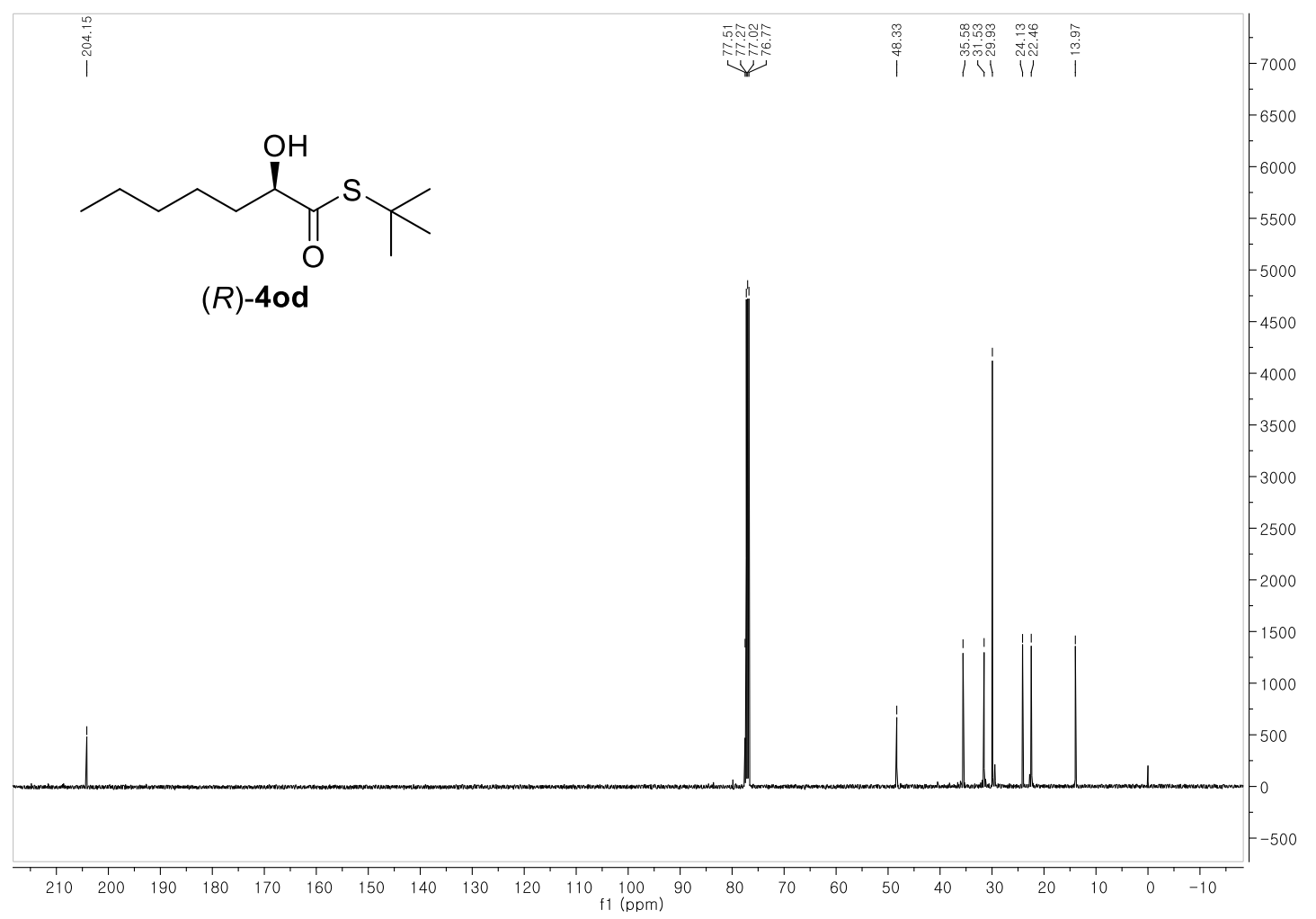

${ }^{13} \mathrm{C}$ NMR spectra of $\mathbf{4 o d}$ 
HPLC Traces

HPLC traces of Scheme 1B

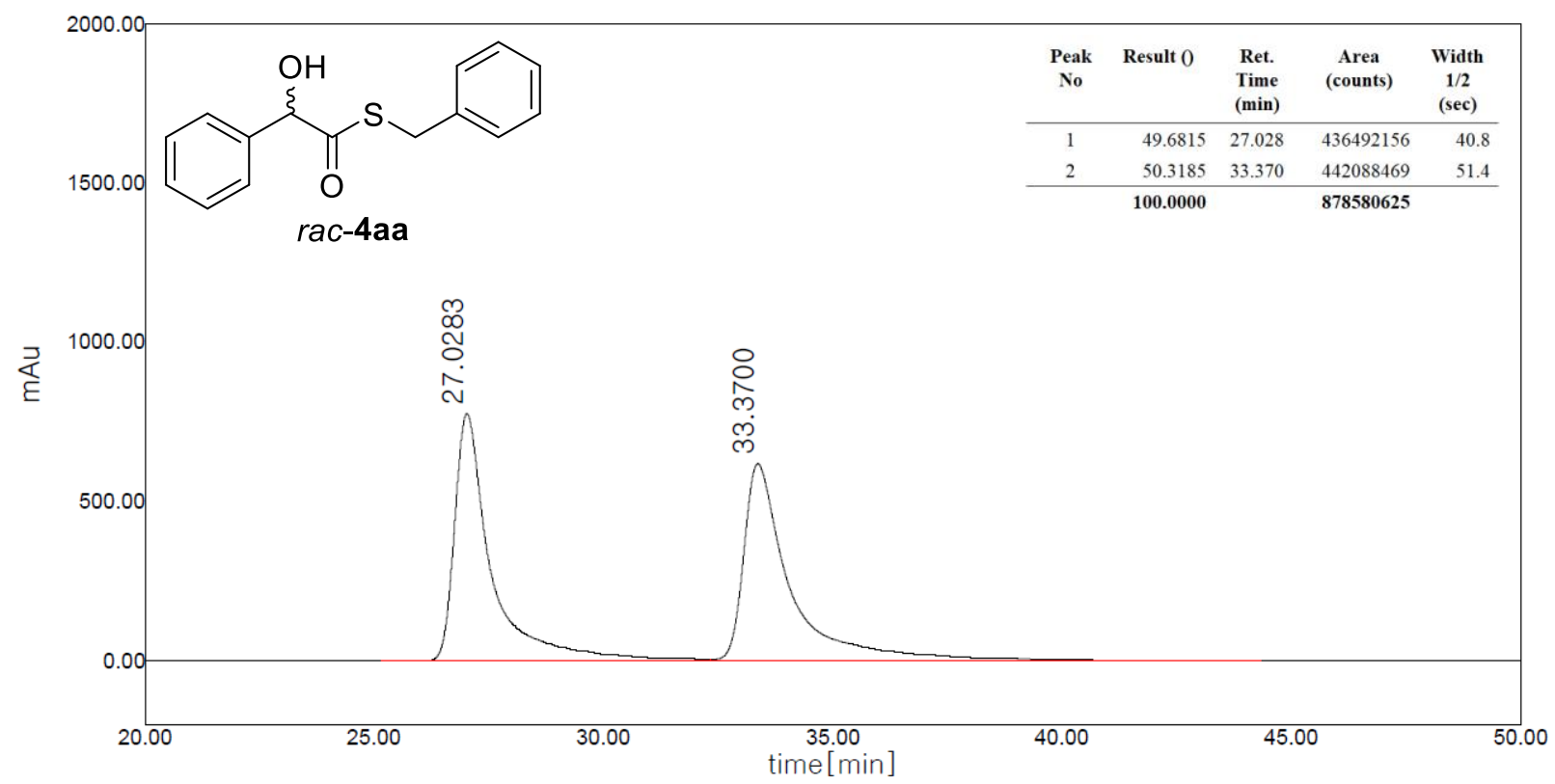

HPLC trace of $r a c-4 a a$

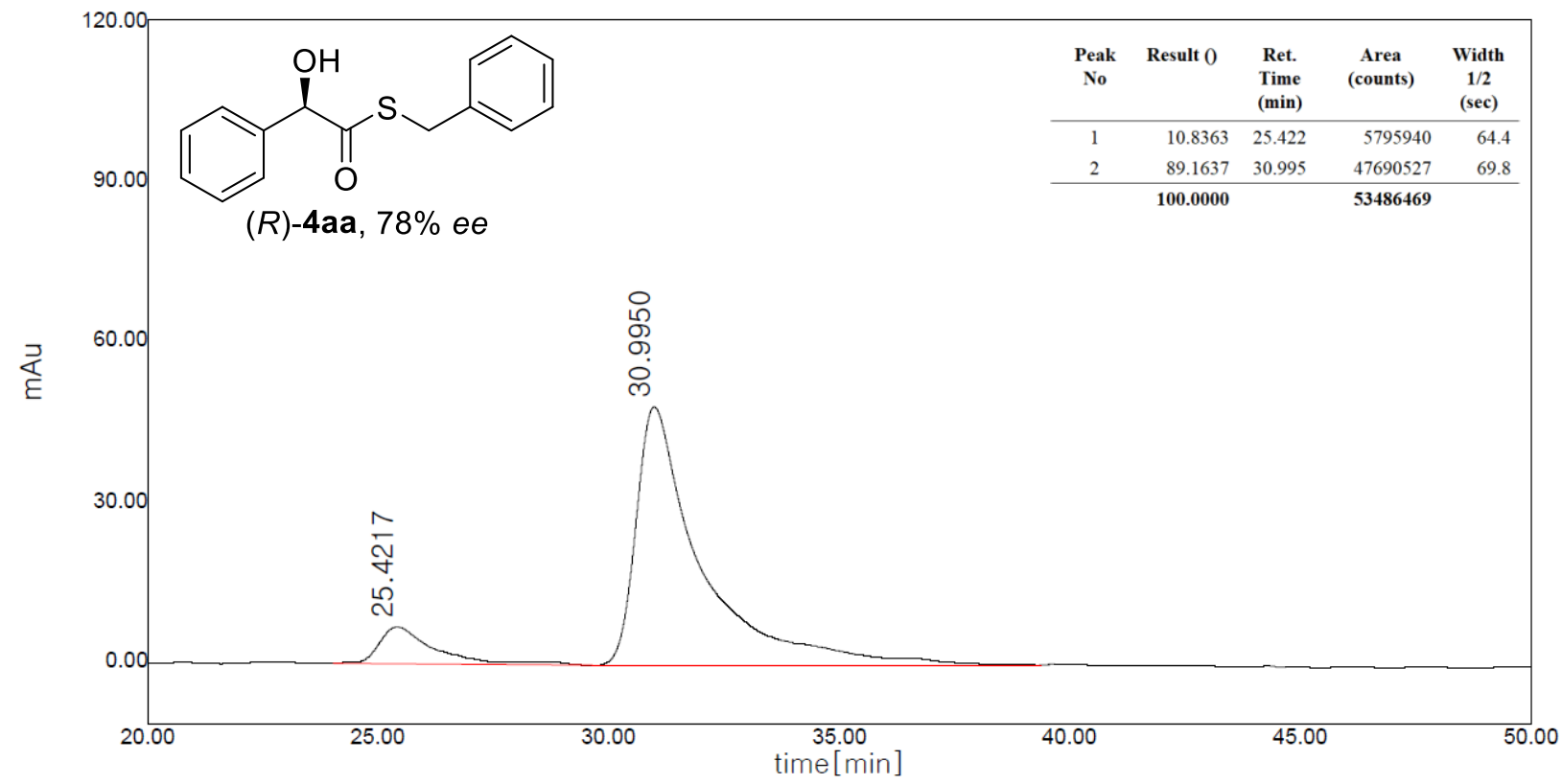

HPLC trace of 4aa (catalyst 5a, in CPME) 


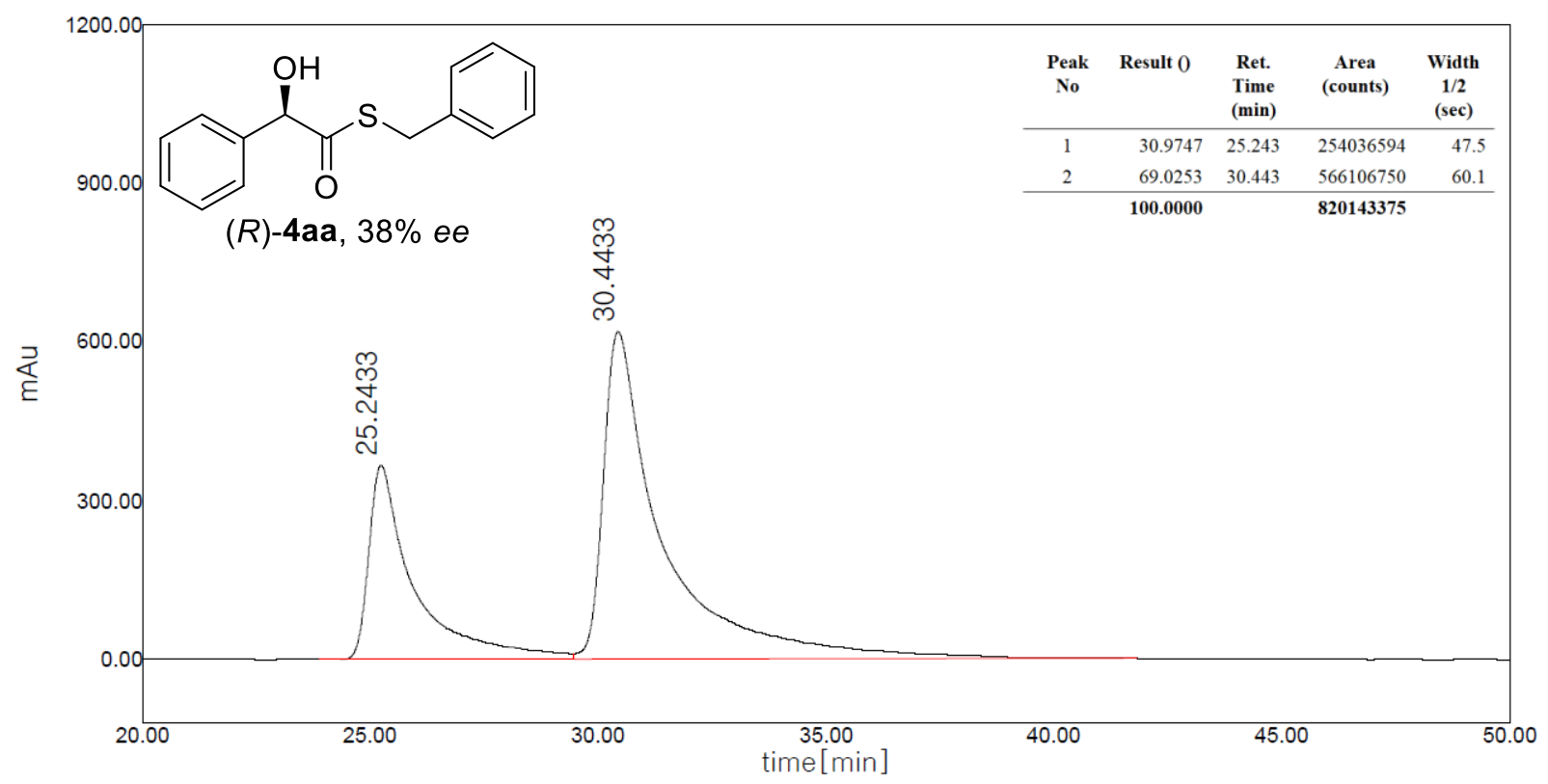

HPLC trace of $4 \mathbf{a a}$ (catalyst 5a, in $\mathrm{H}_{2} \mathrm{O}$ )

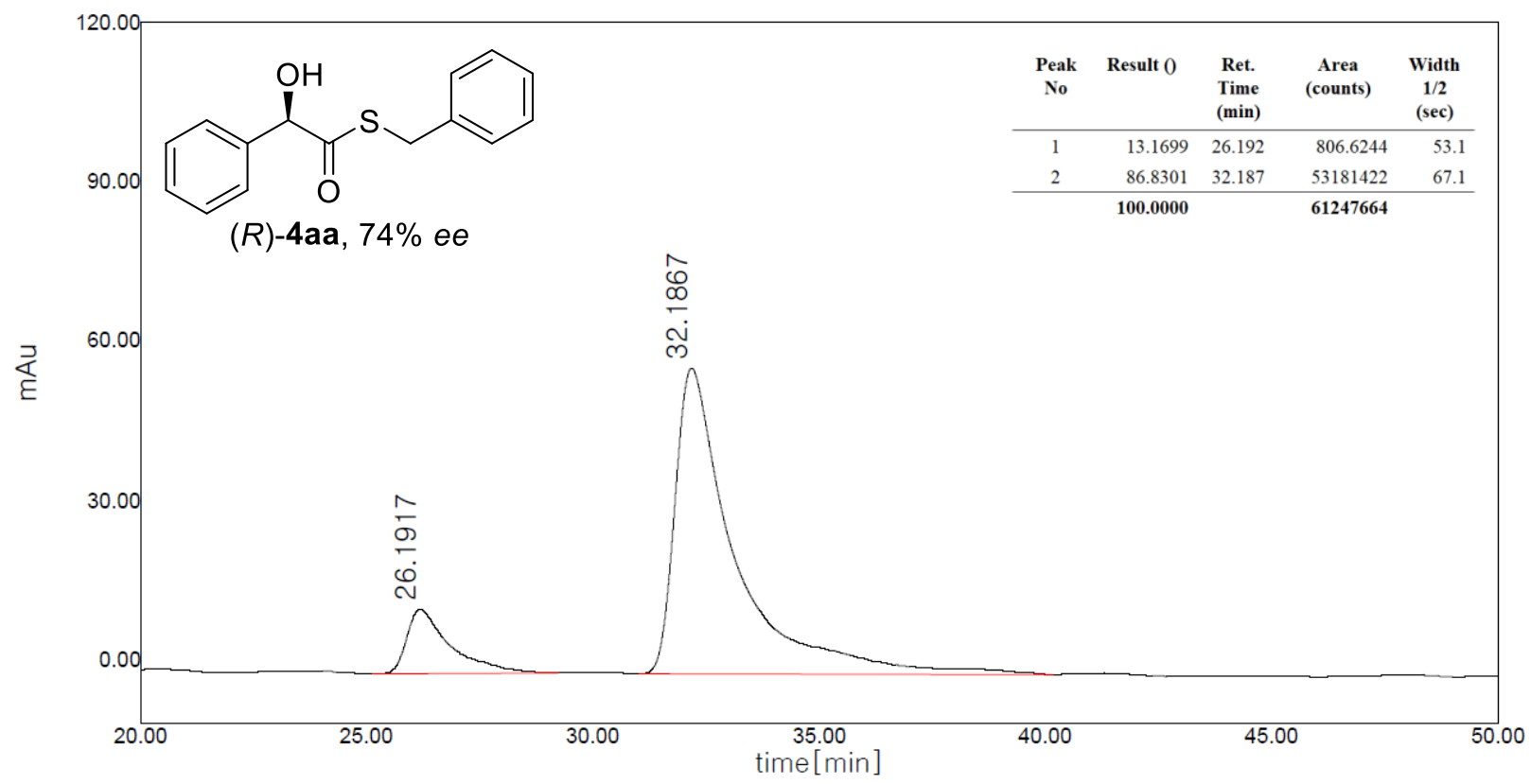

HPLC trace of $\mathbf{4 a a}$ (catalyst 5a, in $\mathrm{H}_{2} \mathrm{O} / \mathrm{CPME}$ ) 


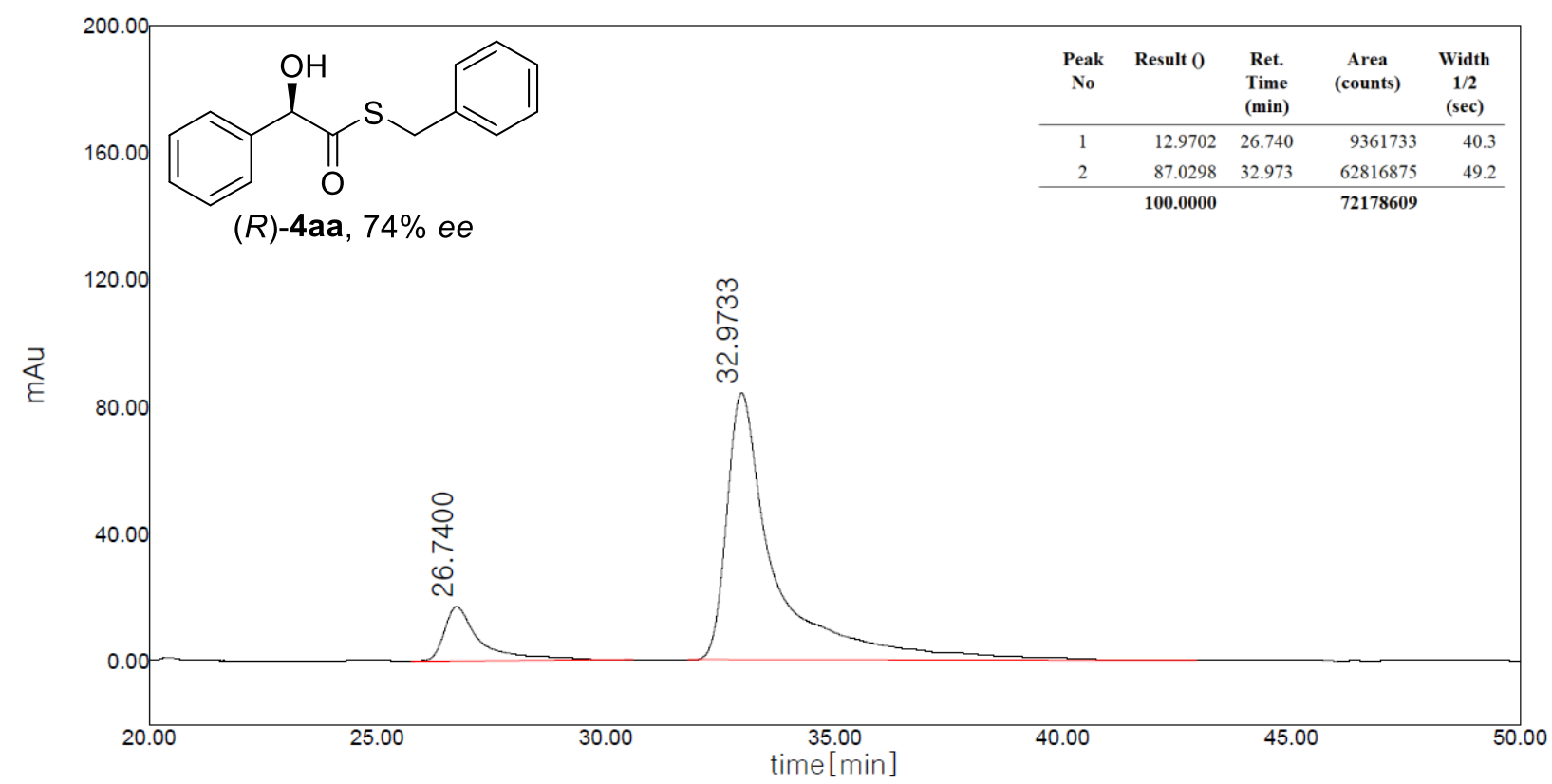

HPLC trace of $\mathbf{4 a a}$ (catalyst 5a, in brine/CPME)

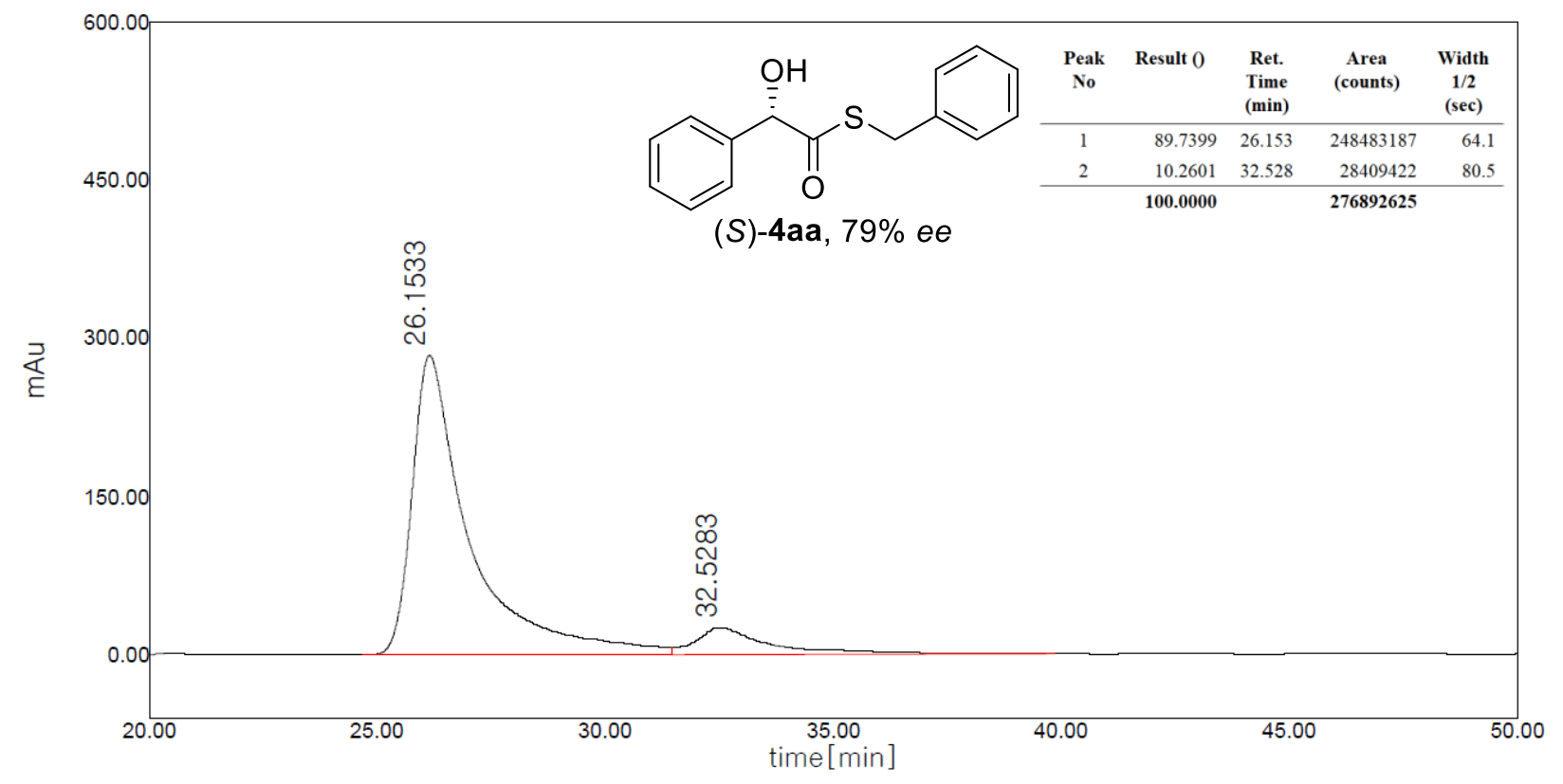

HPLC trace of $\mathbf{4 a a}$ (catalyst $\mathbf{5 b}$, in CPME) 


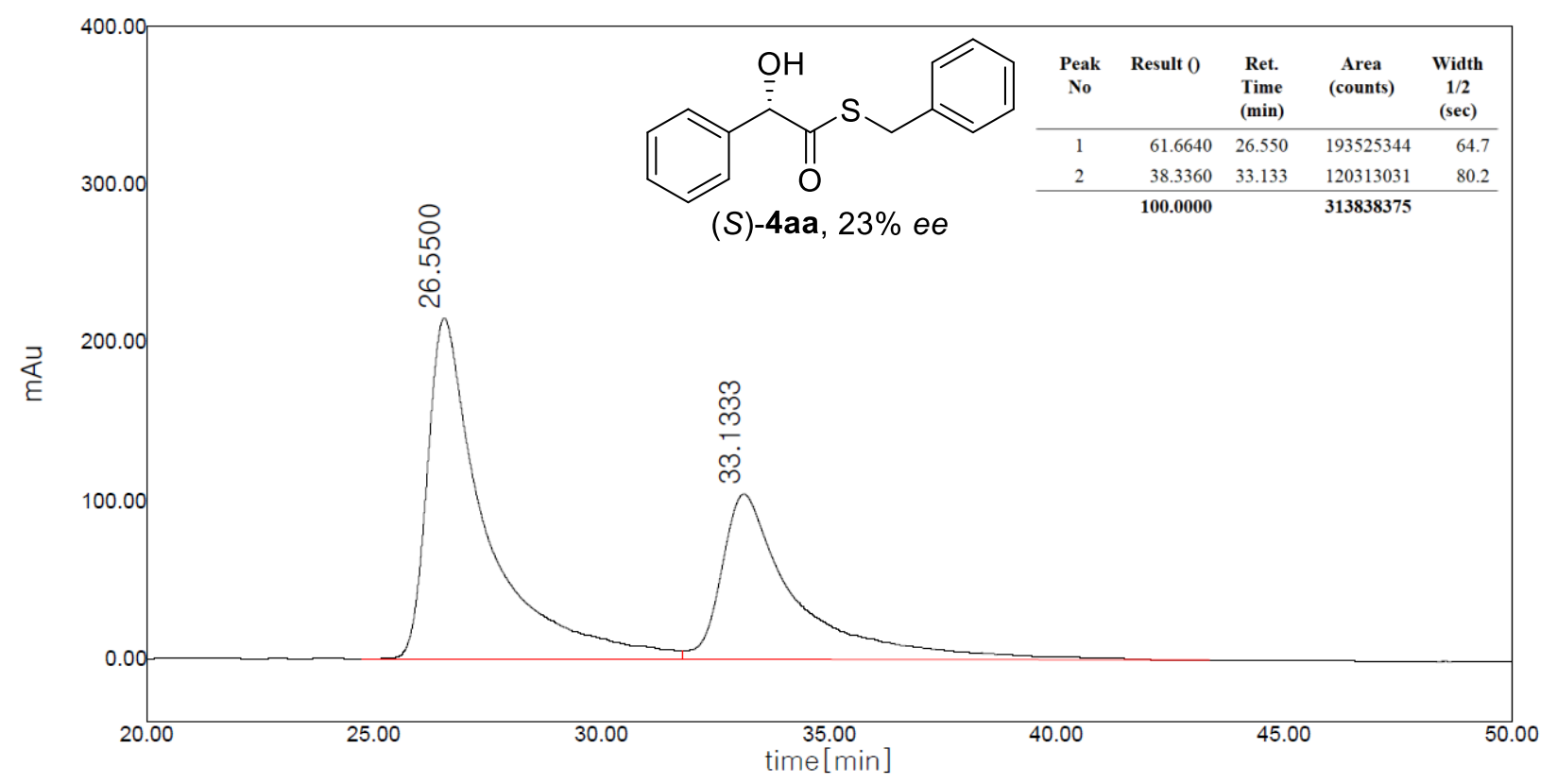

HPLC trace of $\mathbf{4 a a}$ (catalyst $\mathbf{5} \mathbf{b}$, in $\mathrm{H}_{2} \mathrm{O}$ )

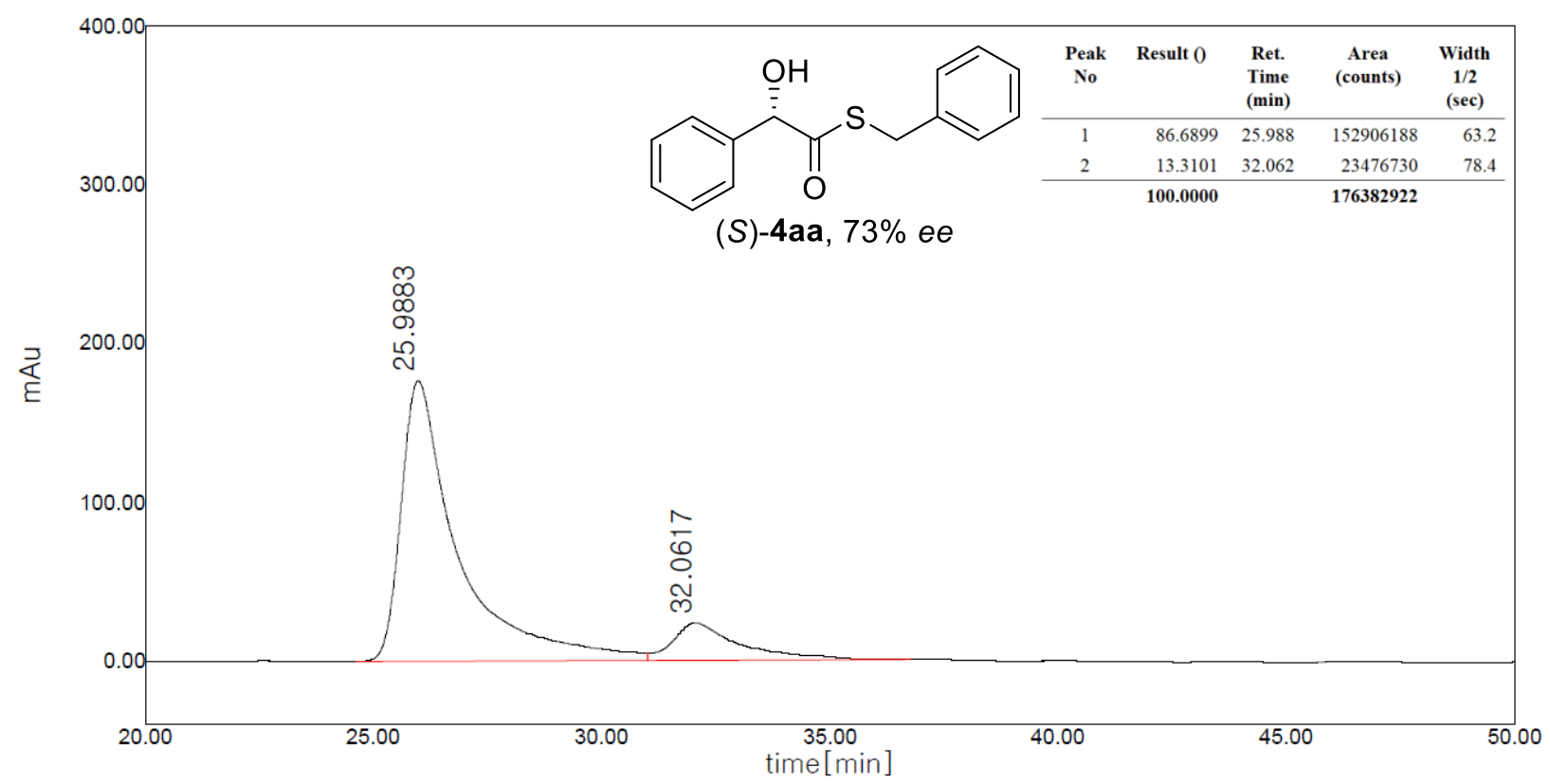

HPLC trace of $\mathbf{4 a a}$ (catalyst $\mathbf{5} \mathbf{b}$, in $\mathrm{H}_{2} \mathrm{O} / \mathrm{CPME}$ ) 


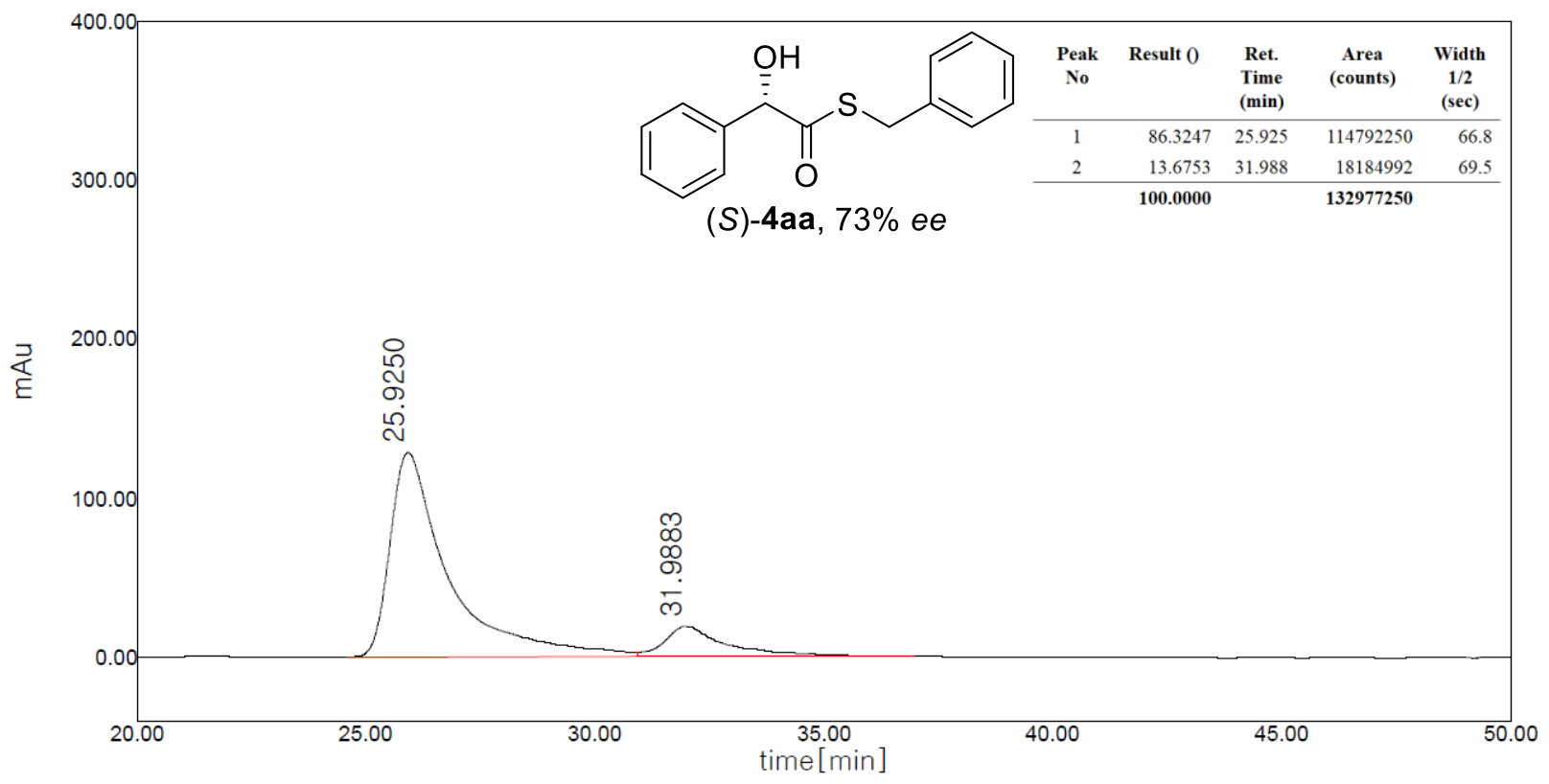

HPLC trace of $\mathbf{4 a a}$ (catalyst $\mathbf{5 b}$, in brine/CPME) 


\section{HPLC traces of Table S1}

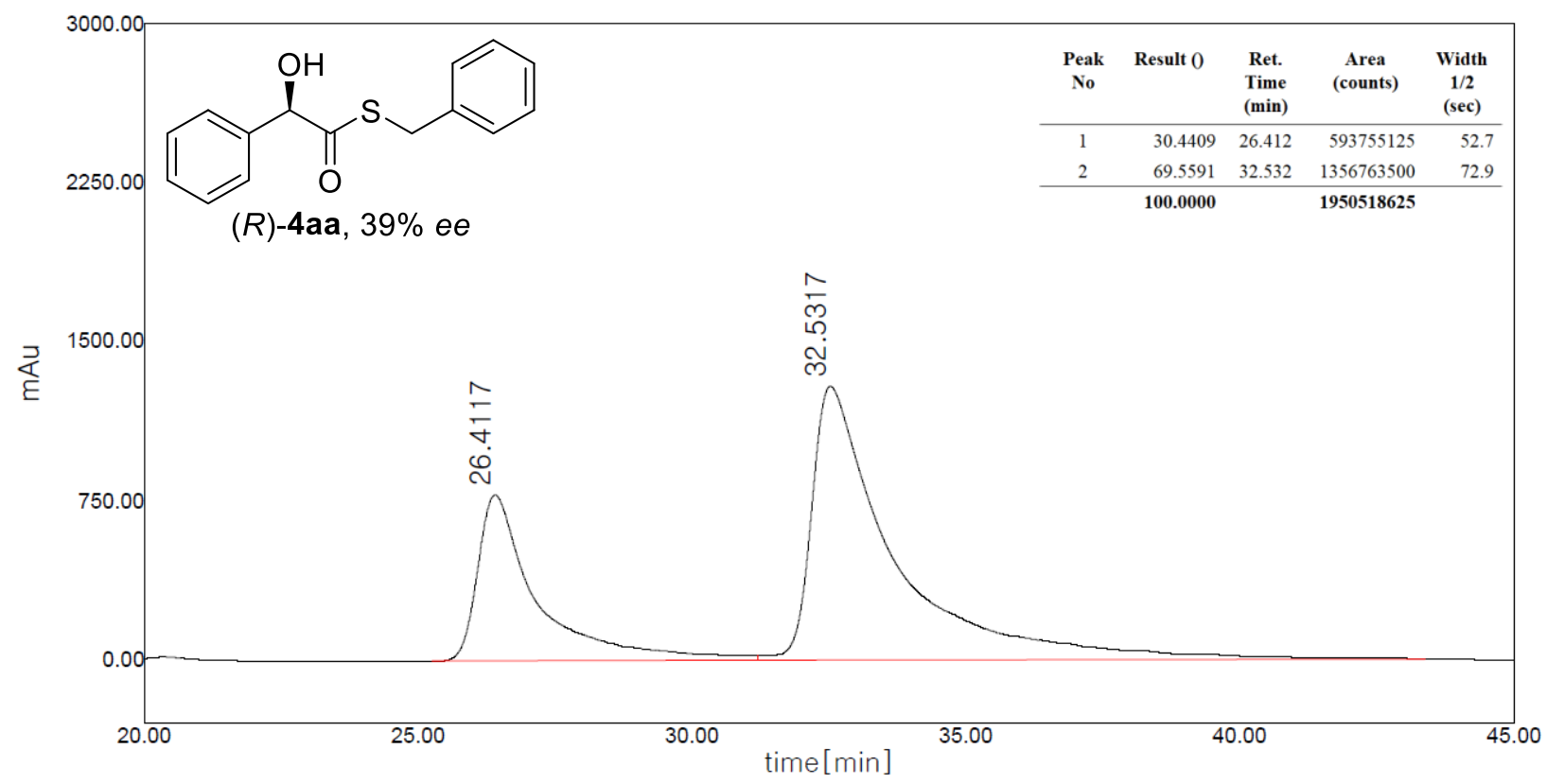

HPLC trace of 4aa (in toluene)

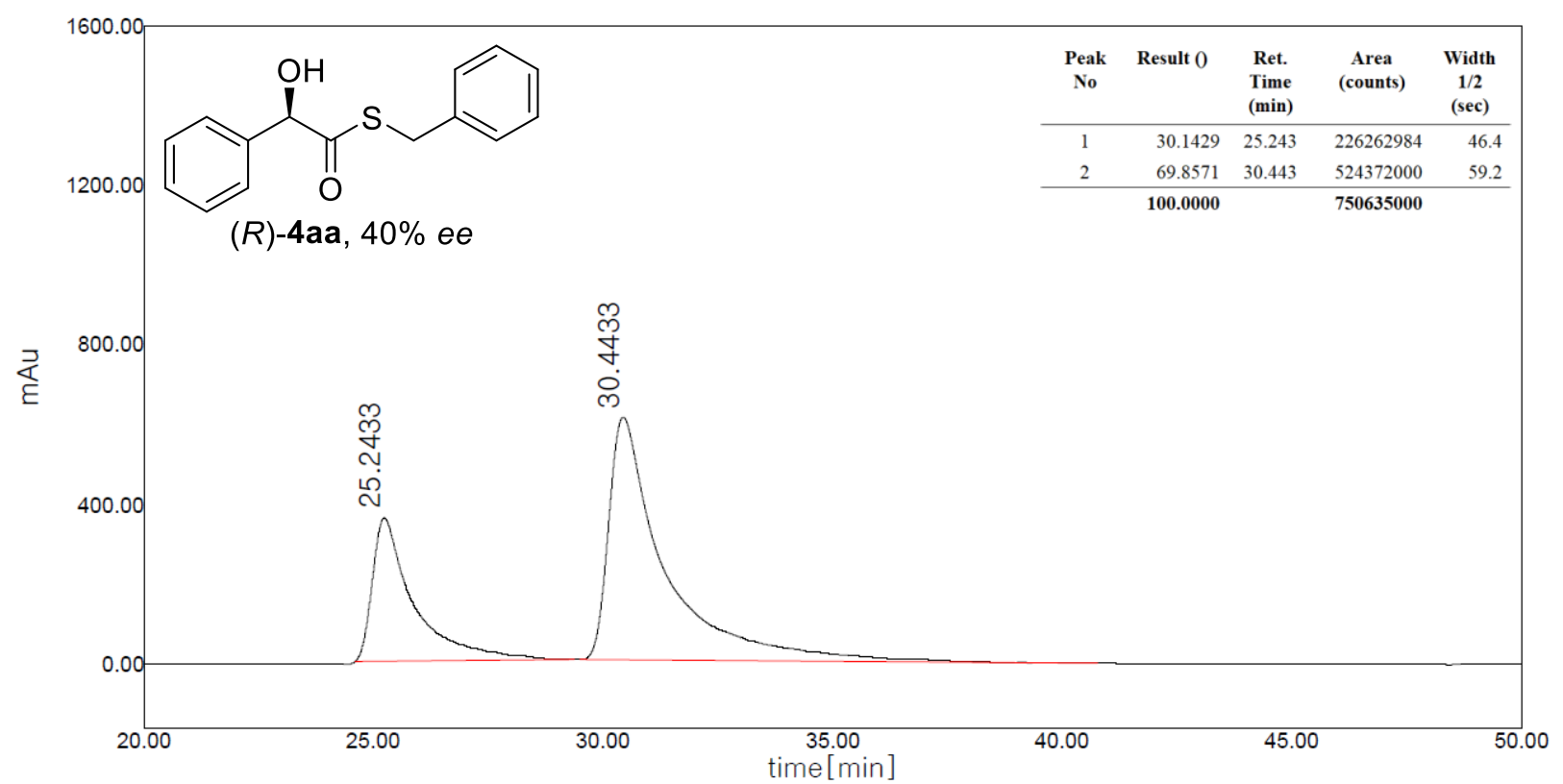

HPLC trace of 4aa (in $o$-xylene) 


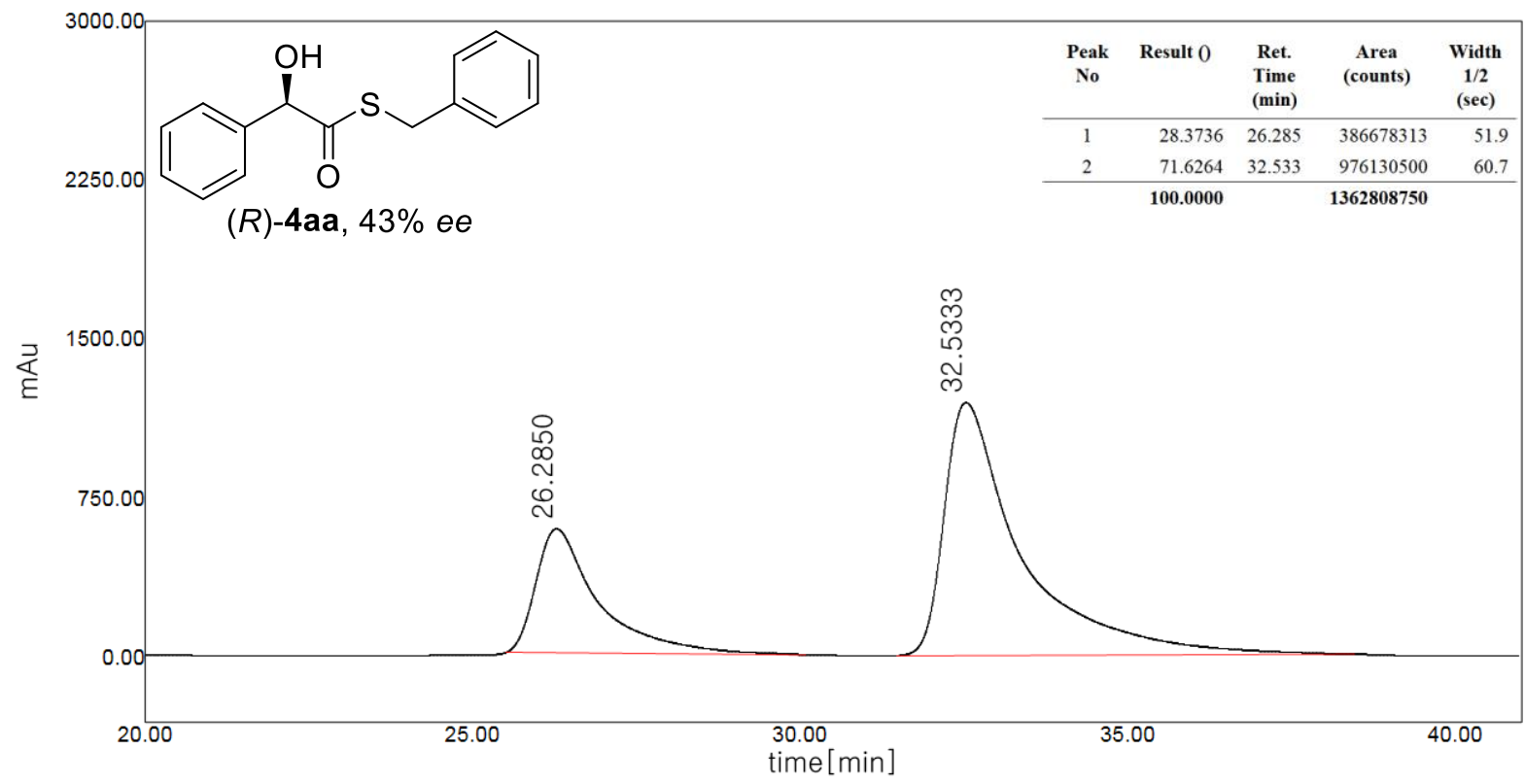

HPLC trace of 4aa (in $m$-xylene)

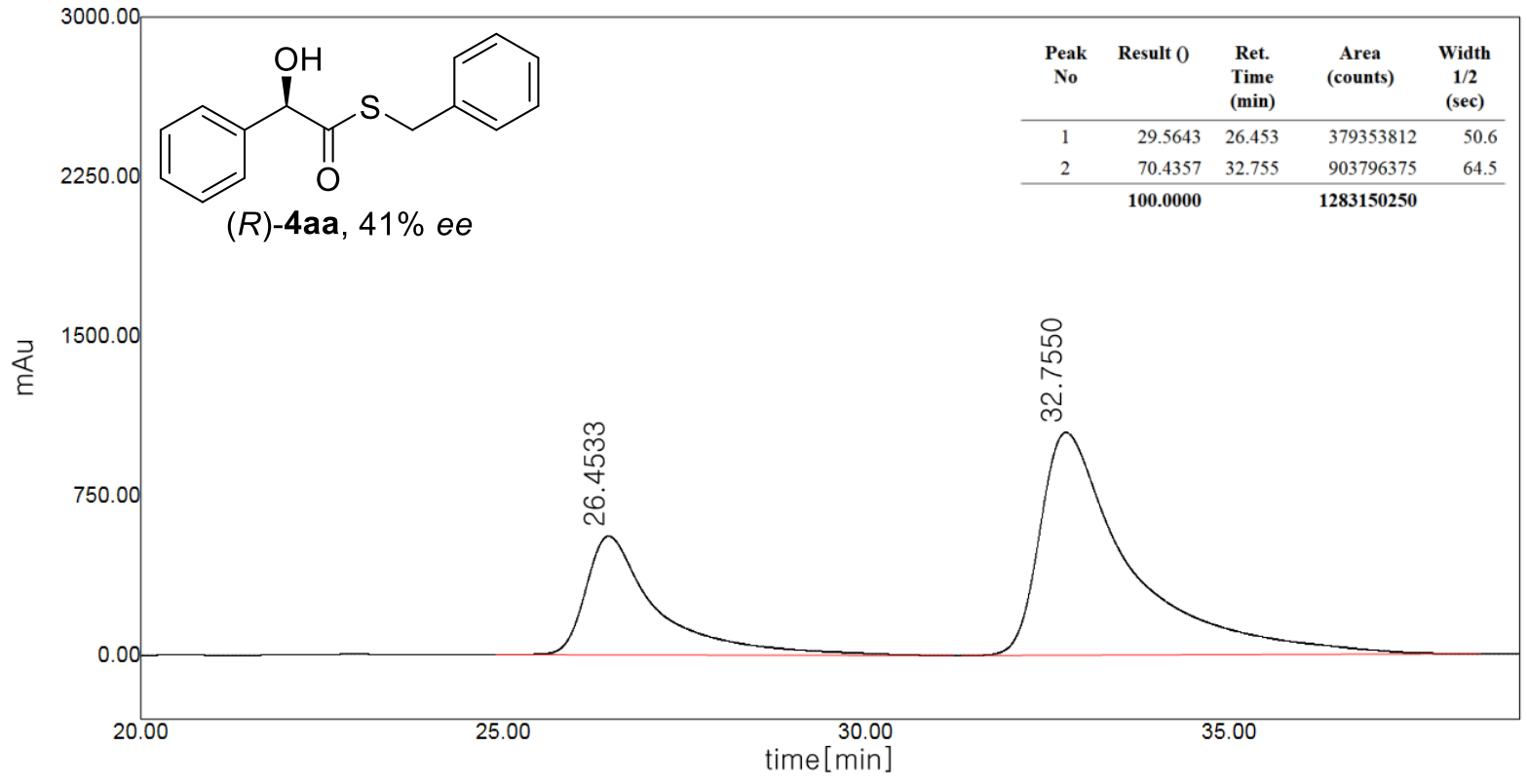

HPLC trace of 4aa (in $p$-xylene) 


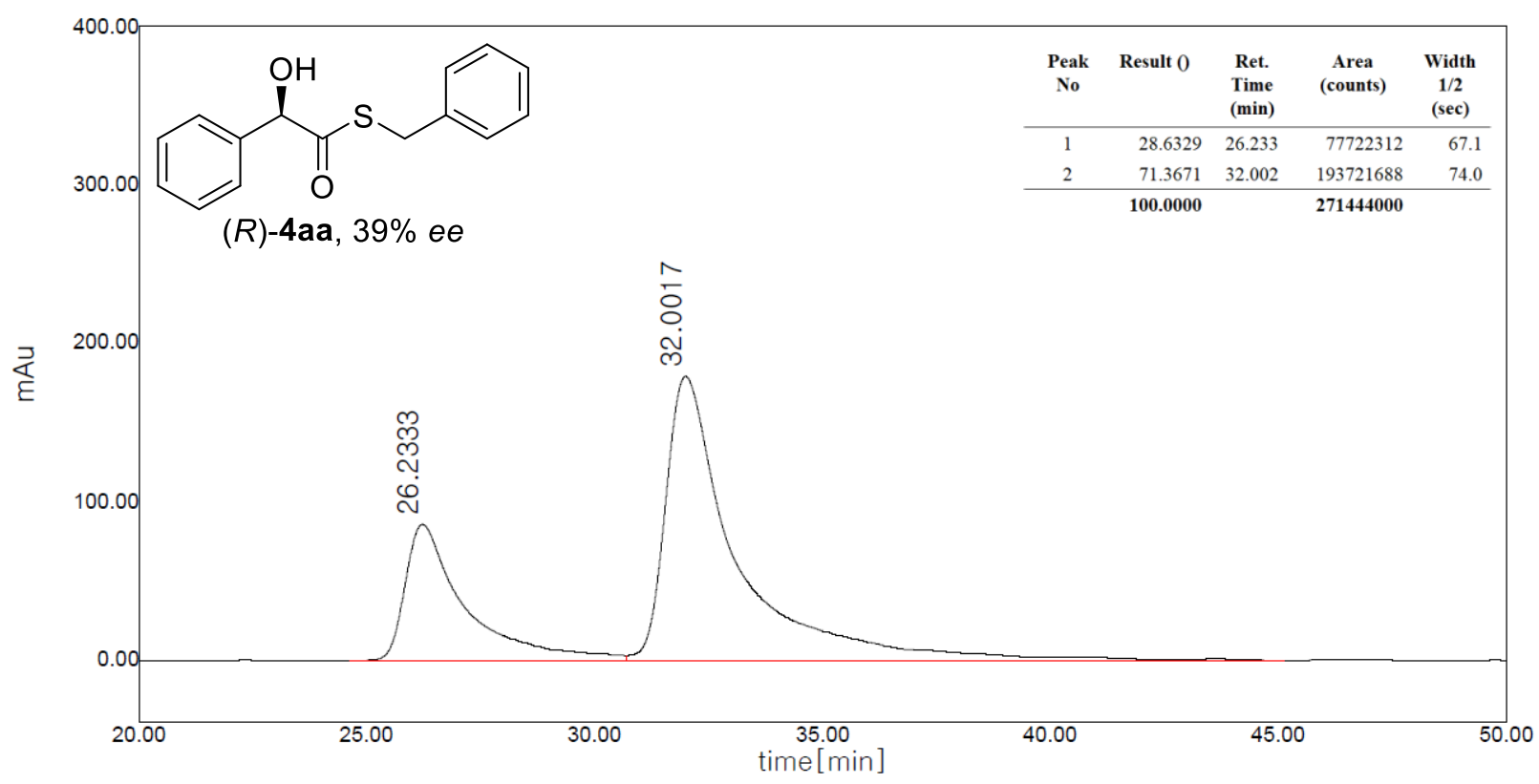

HPLC trace of 4aa (in chlorobenzene)

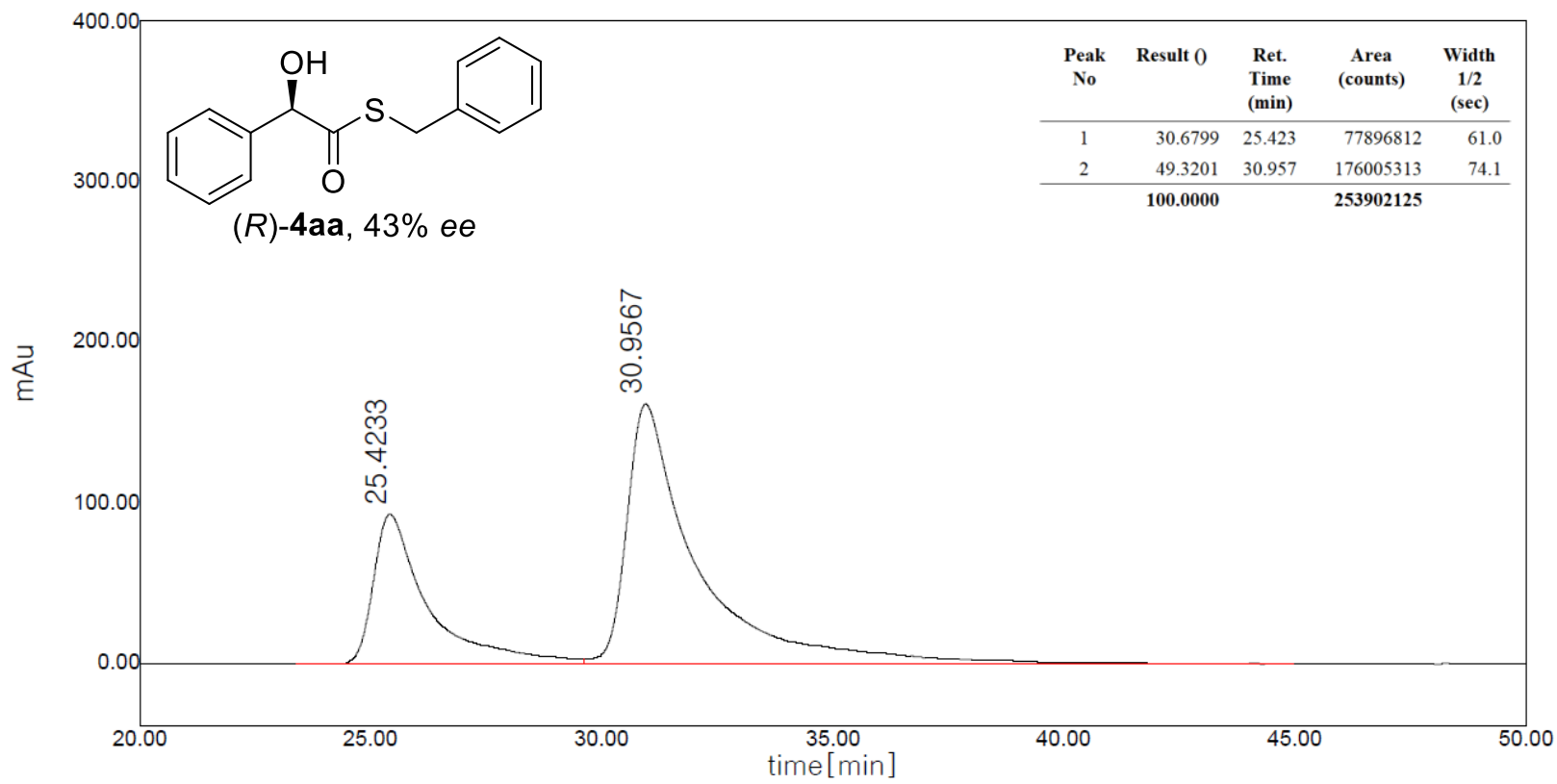

HPLC trace of 4aa (in bromobenzene) 


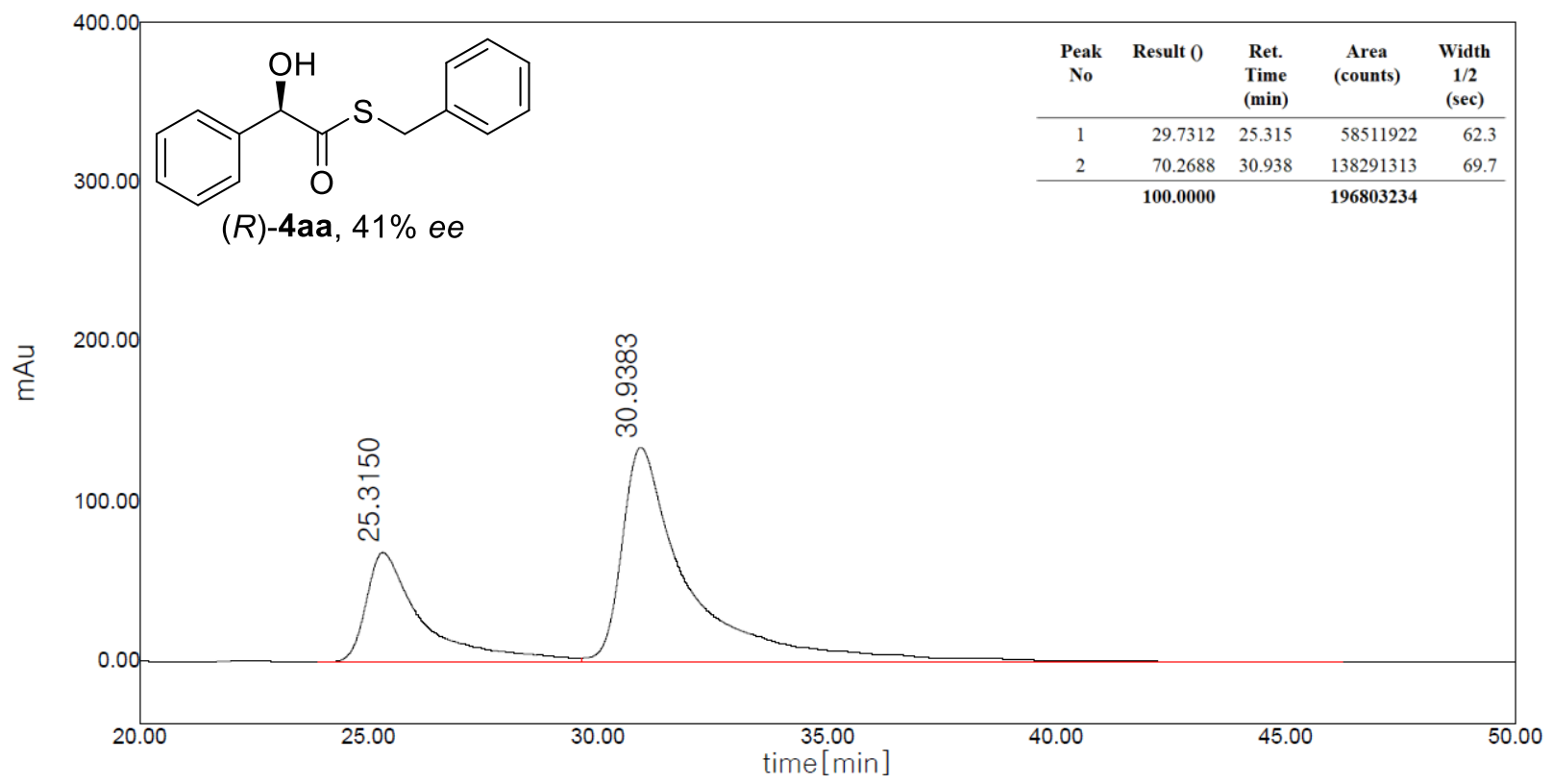

HPLC trace of 4aa (in 1,2-dibromobenzene)

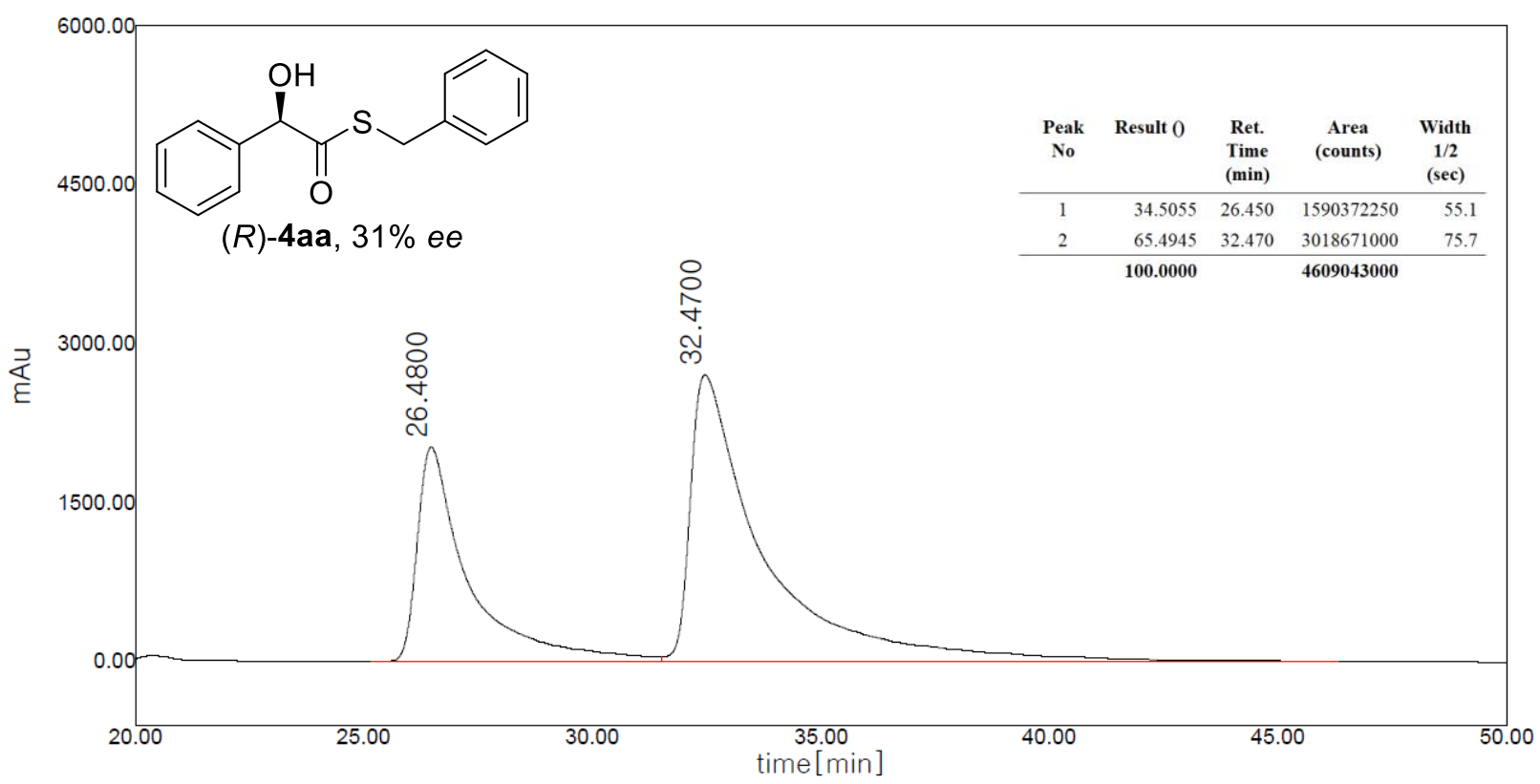

HPLC trace of $\mathbf{4 a a}$ (in $\mathrm{CH}_{2} \mathrm{Cl}_{2}$ ) 


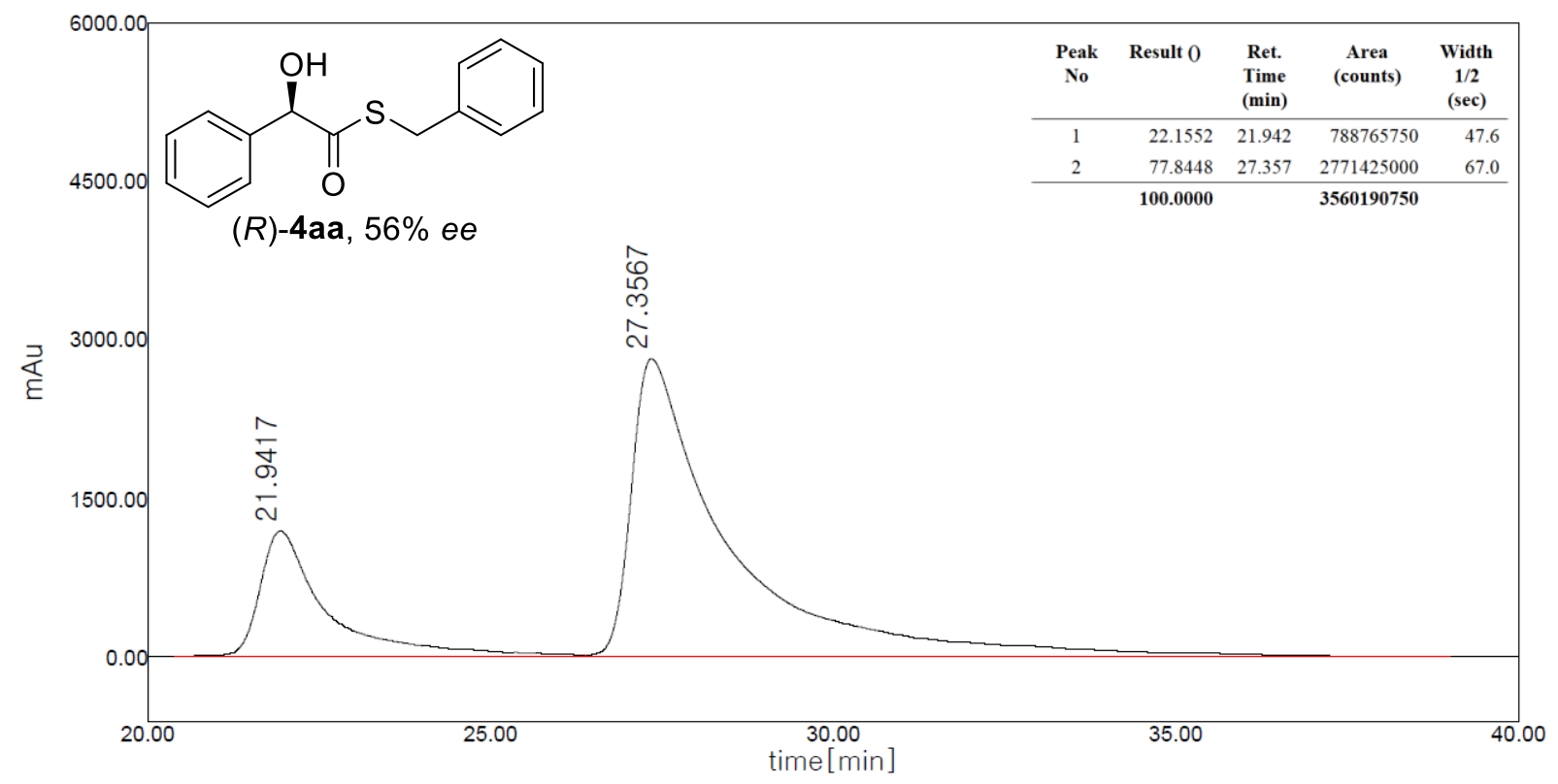

HPLC trace of 4aa (in diethyl ether)

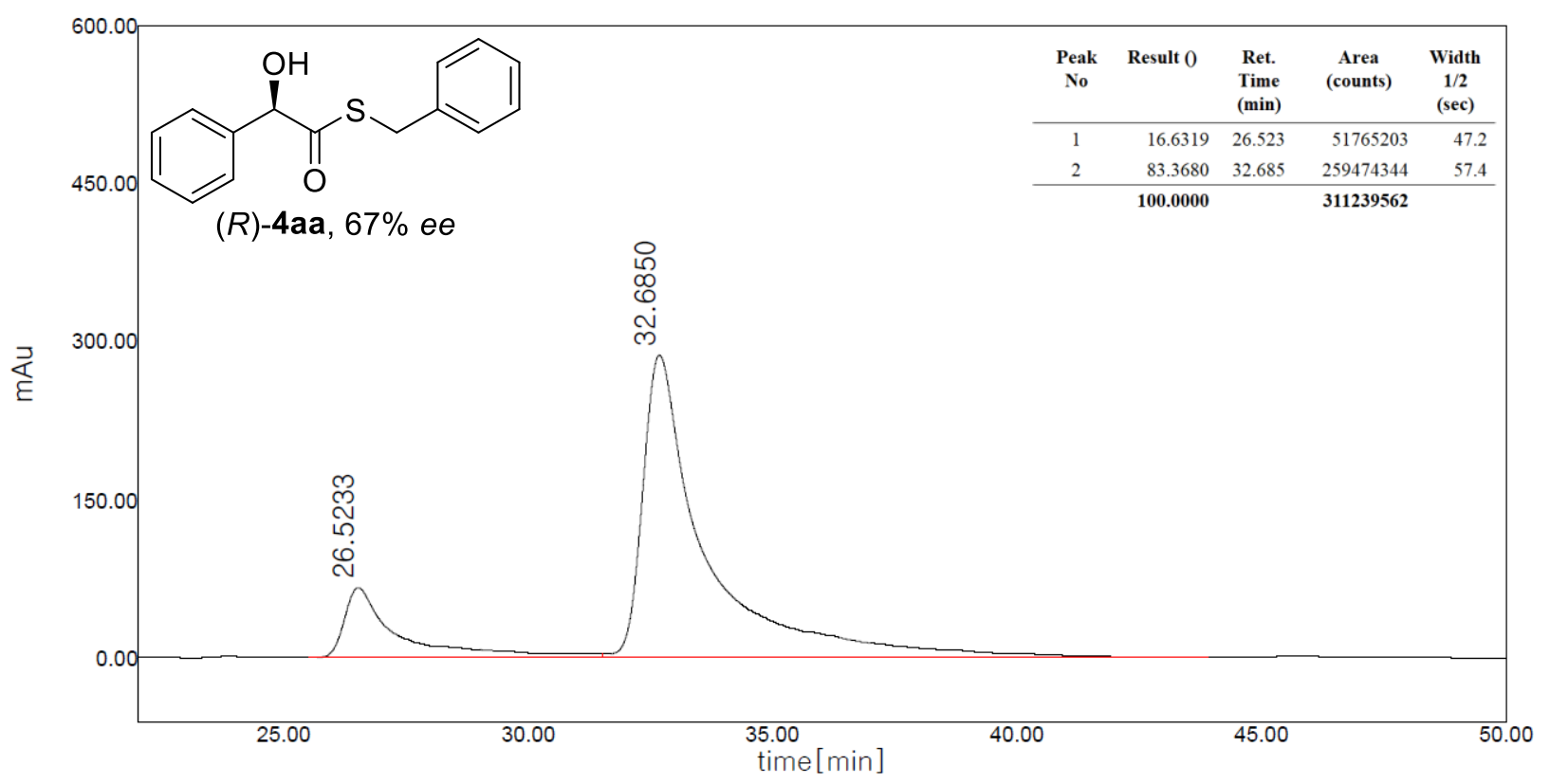

HPLC trace of 4aa (in diisopropyl ether) 


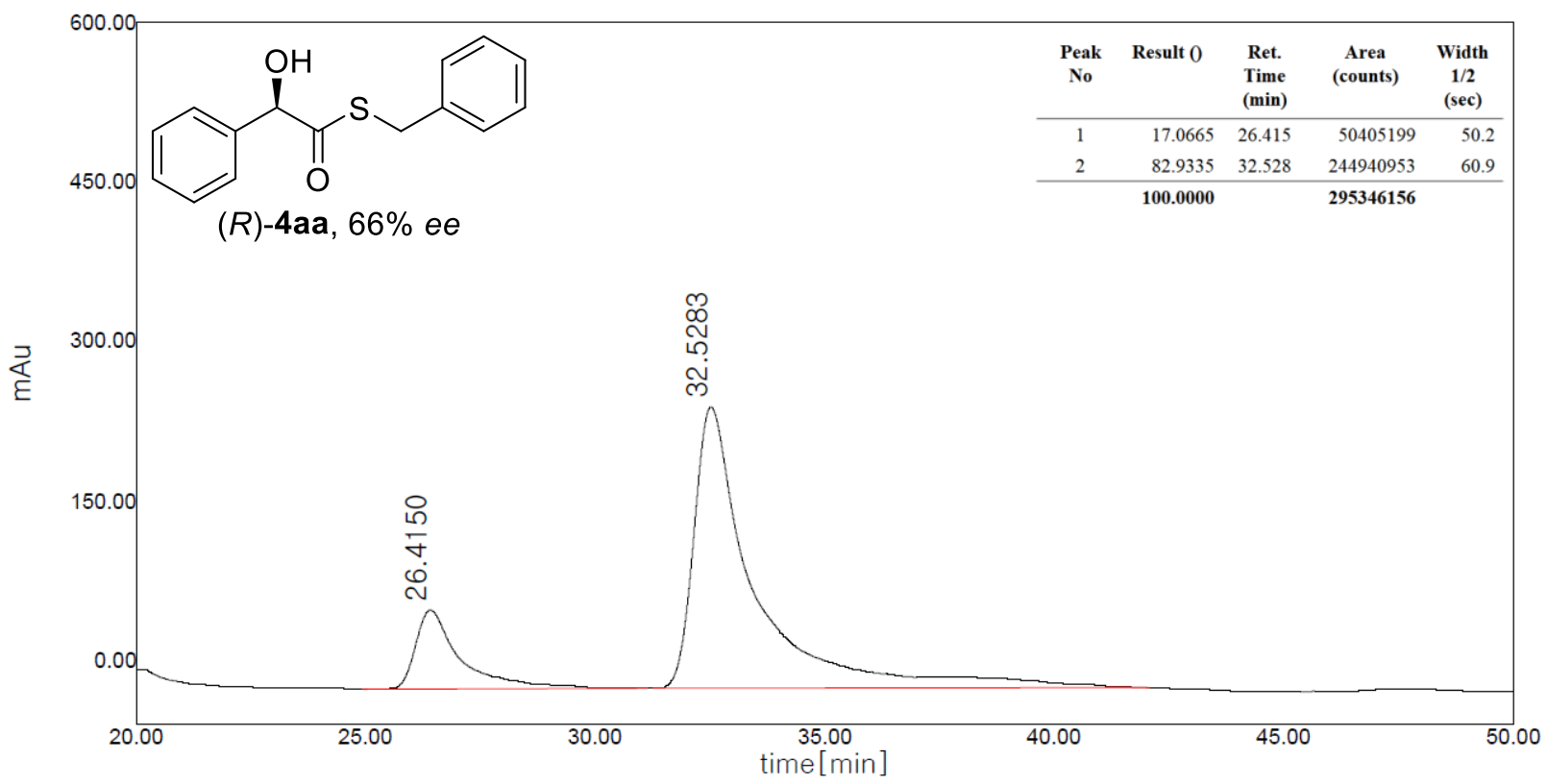

HPLC trace of 4aa (in dibutyl ether)

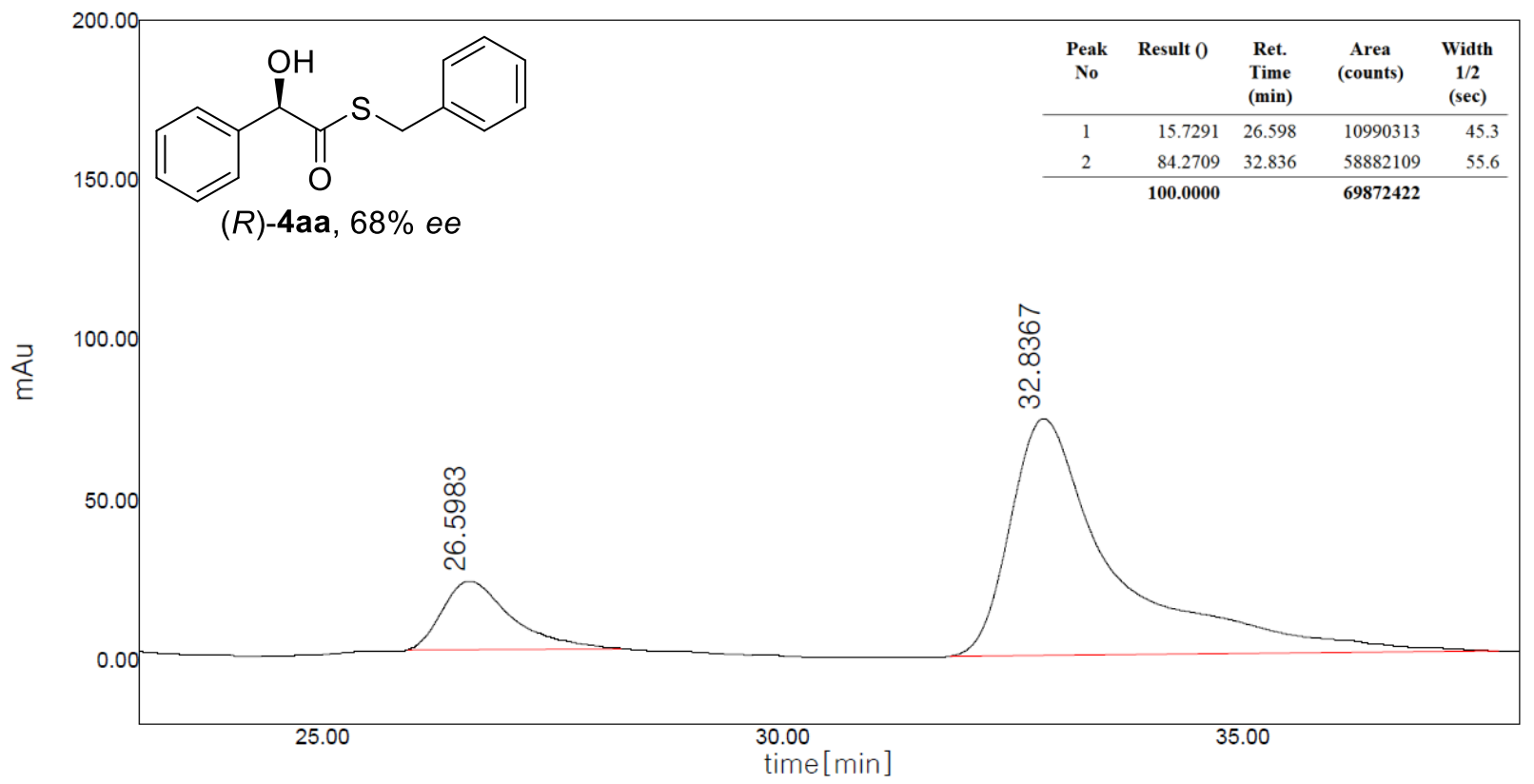

HPLC trace of 4aa (in dipentyl ether) 


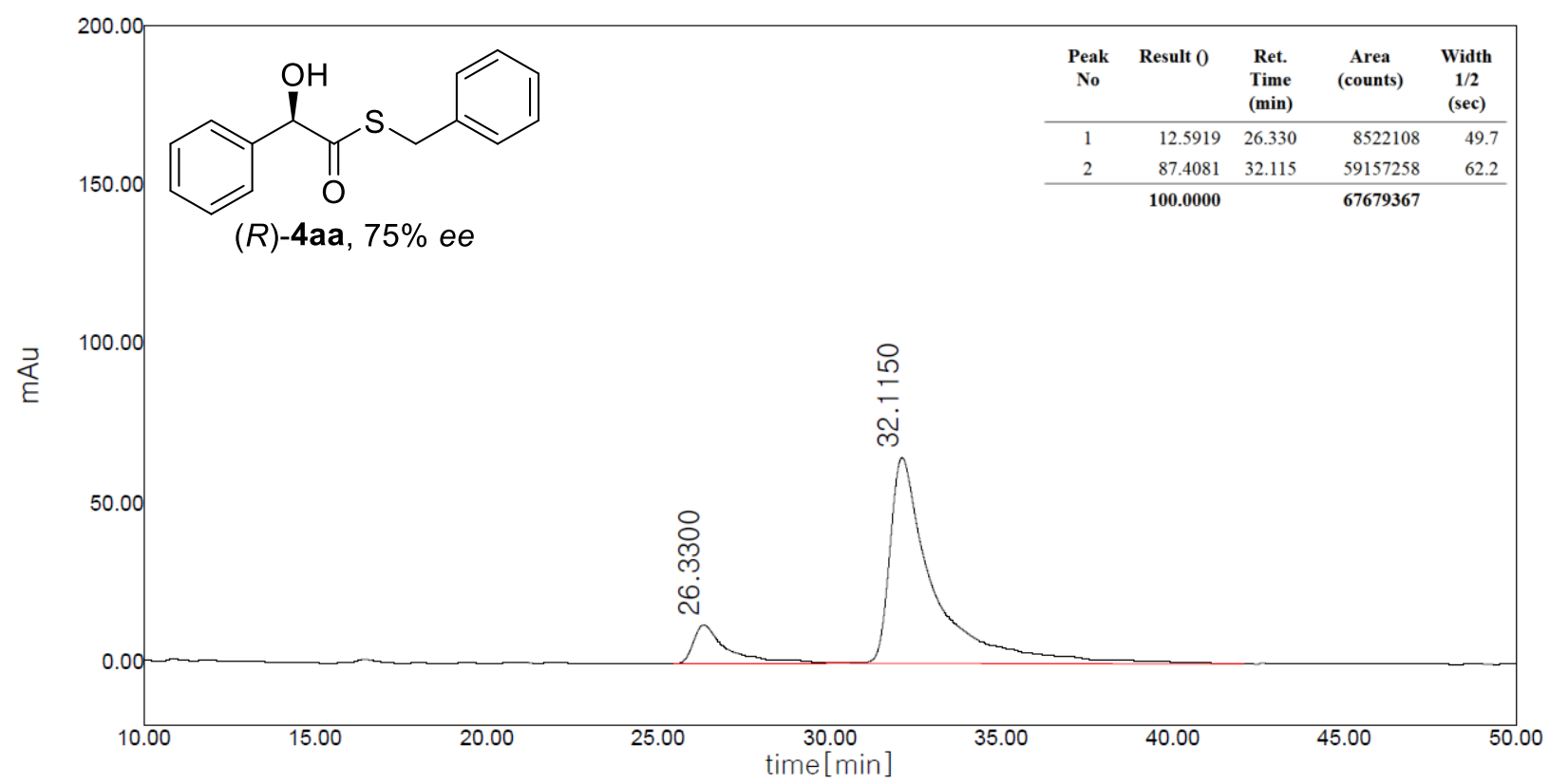

HPLC trace of 4aa (in MTBE)

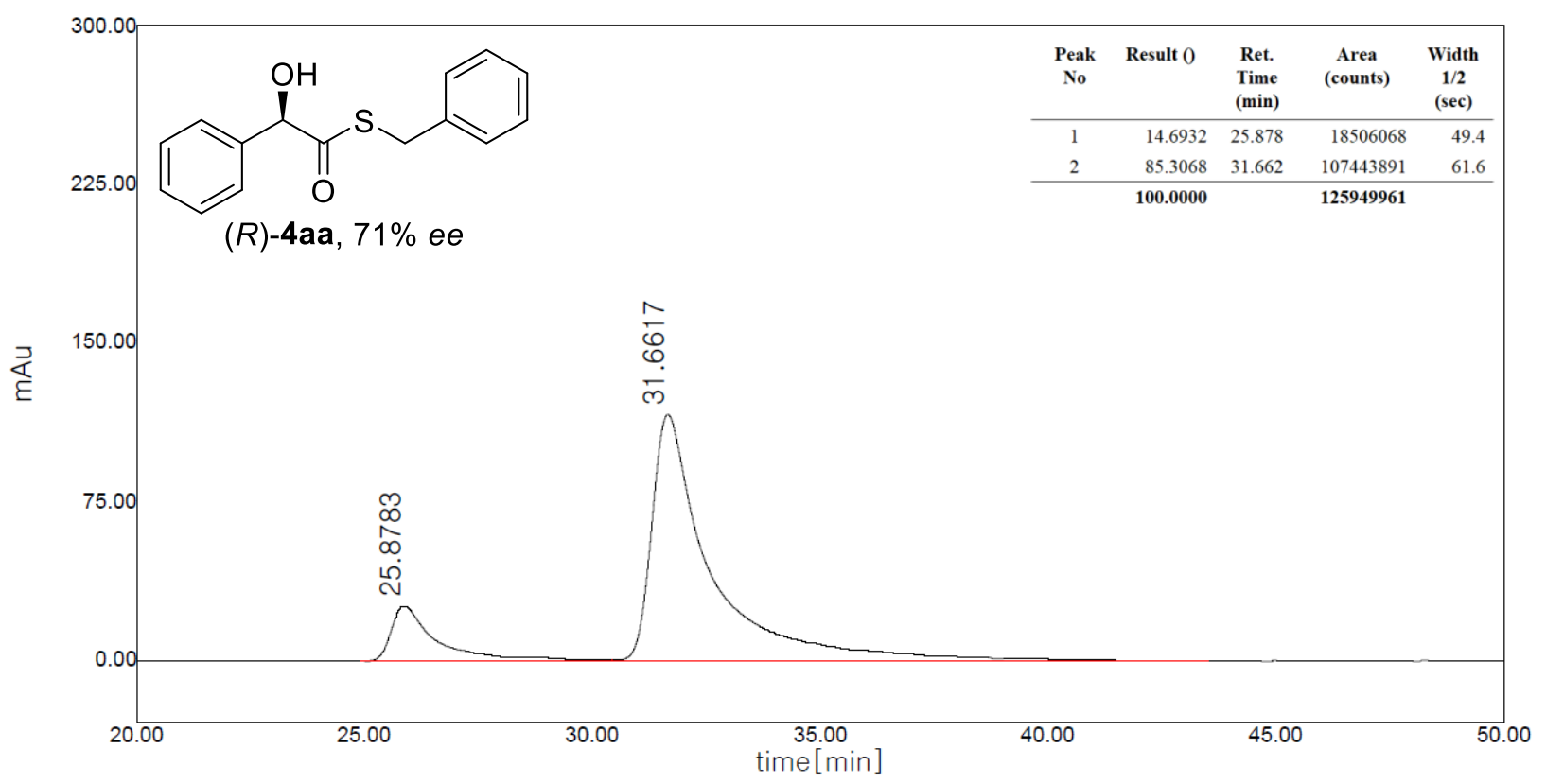

HPLC trace of 4aa (in methoxy cyclohexane) 


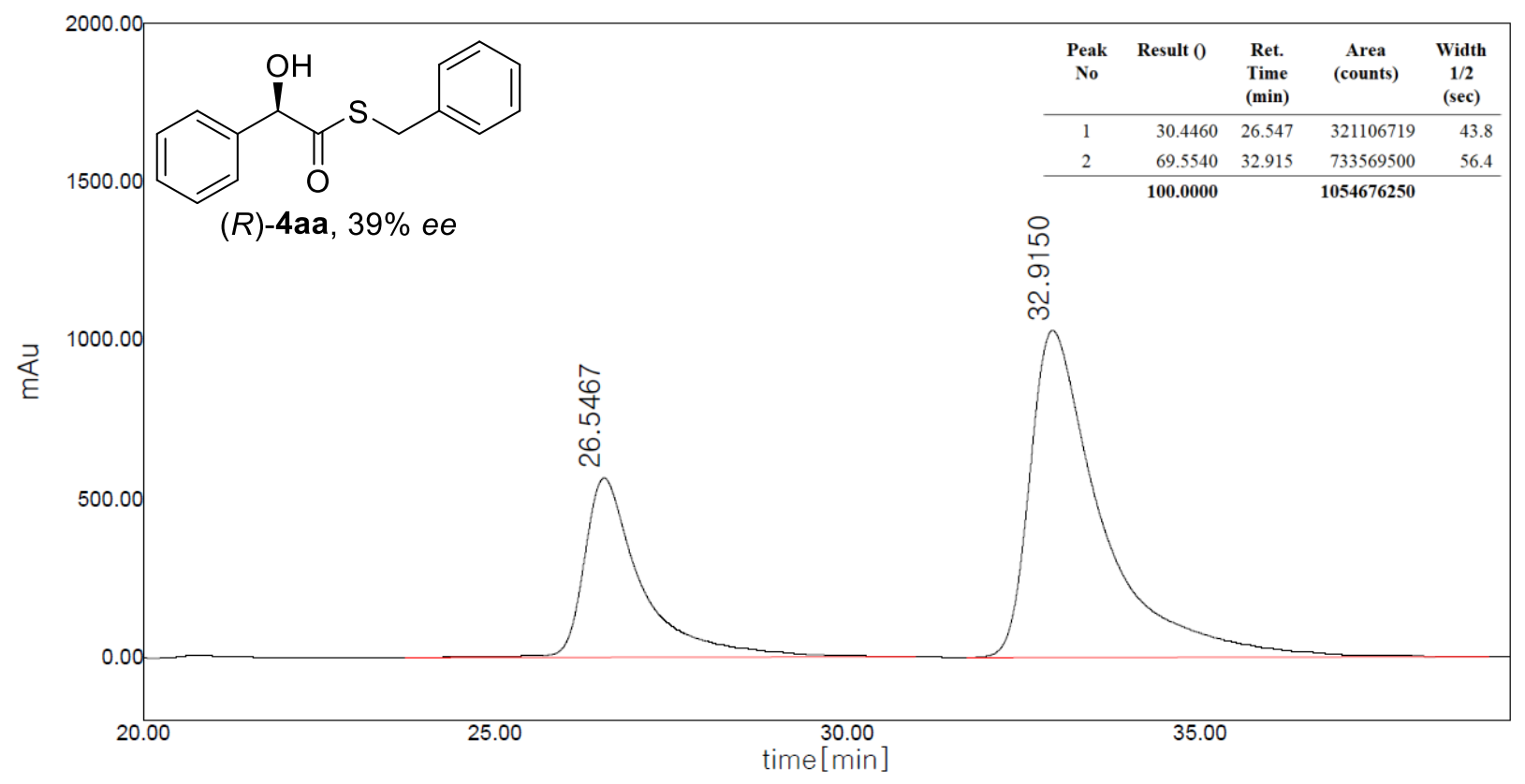

HPLC trace of 4aa (in methyl propargyl ether)

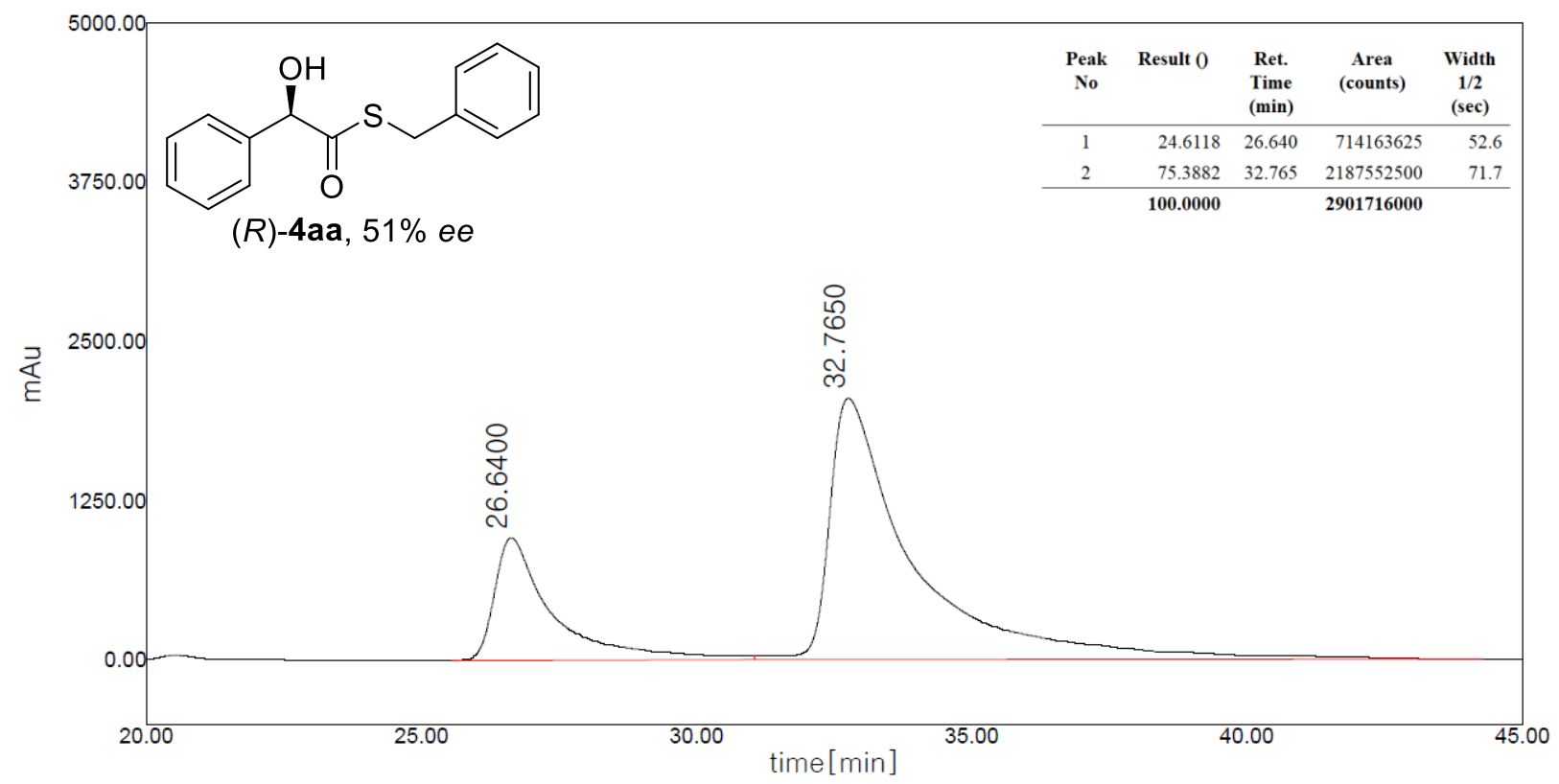

HPLC trace of 4aa (in THF) 


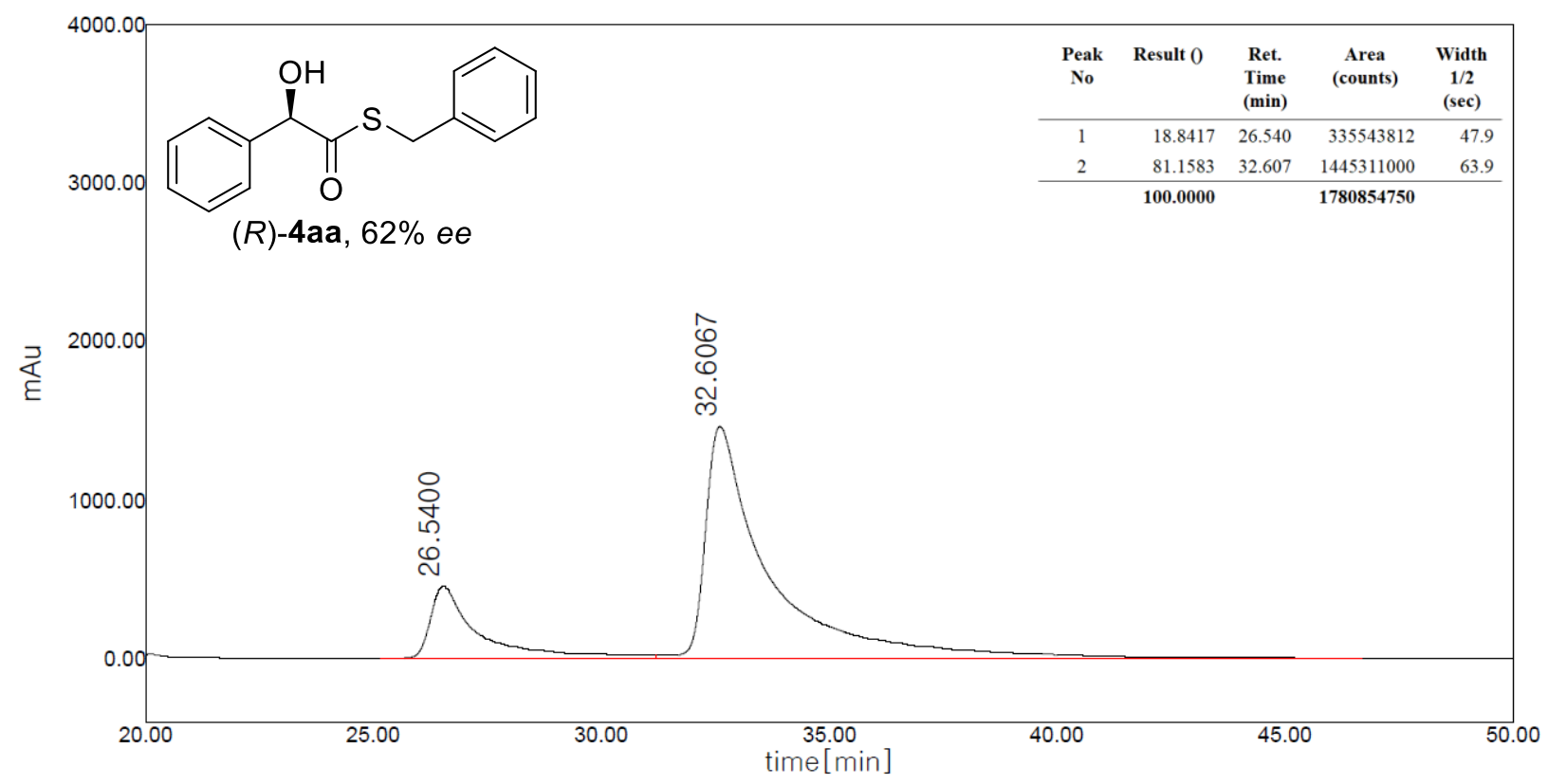

HPLC trace of 4aa (in 2-methyl THF)

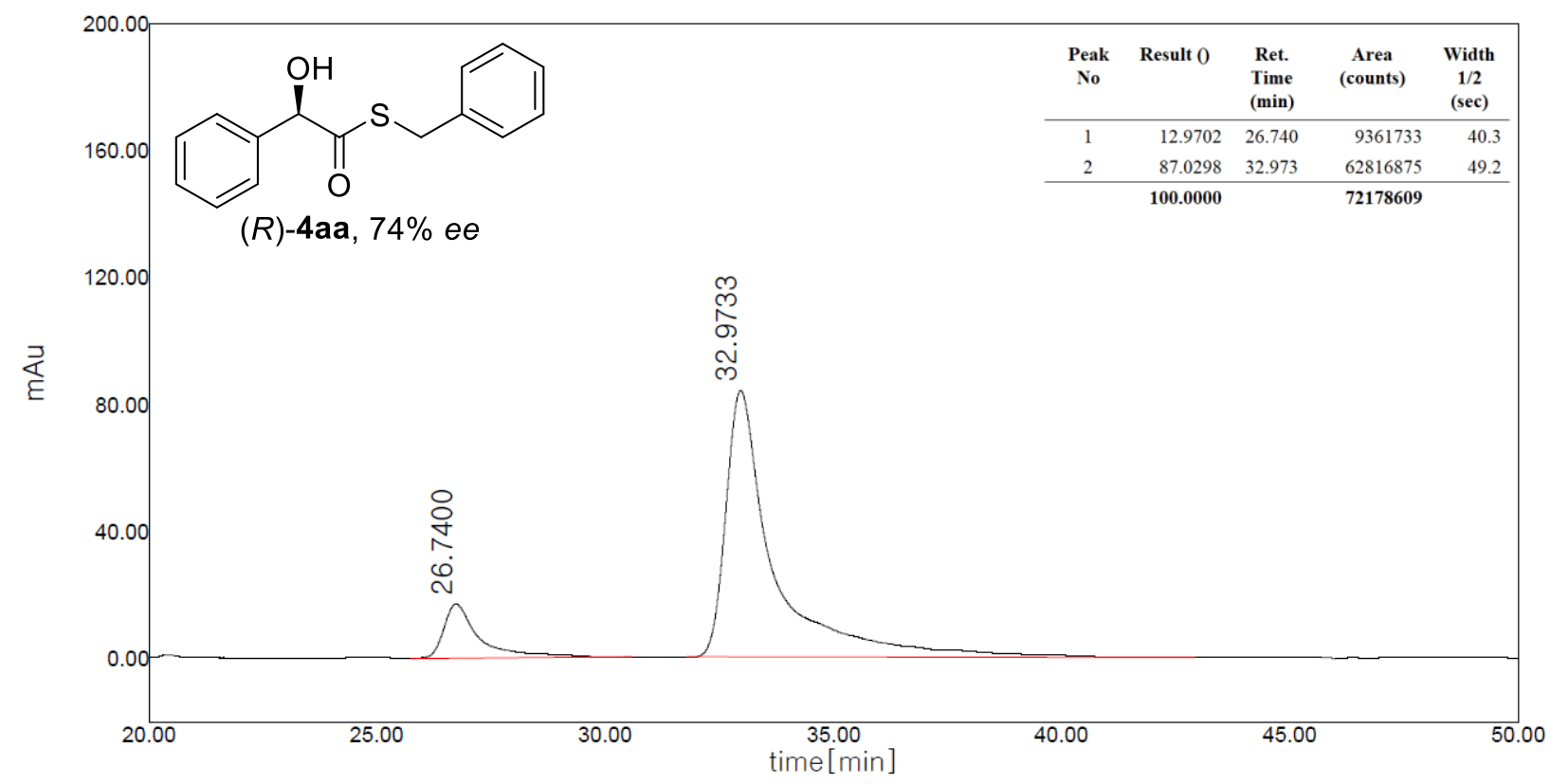

HPLC trace of 4aa (in CPME) 


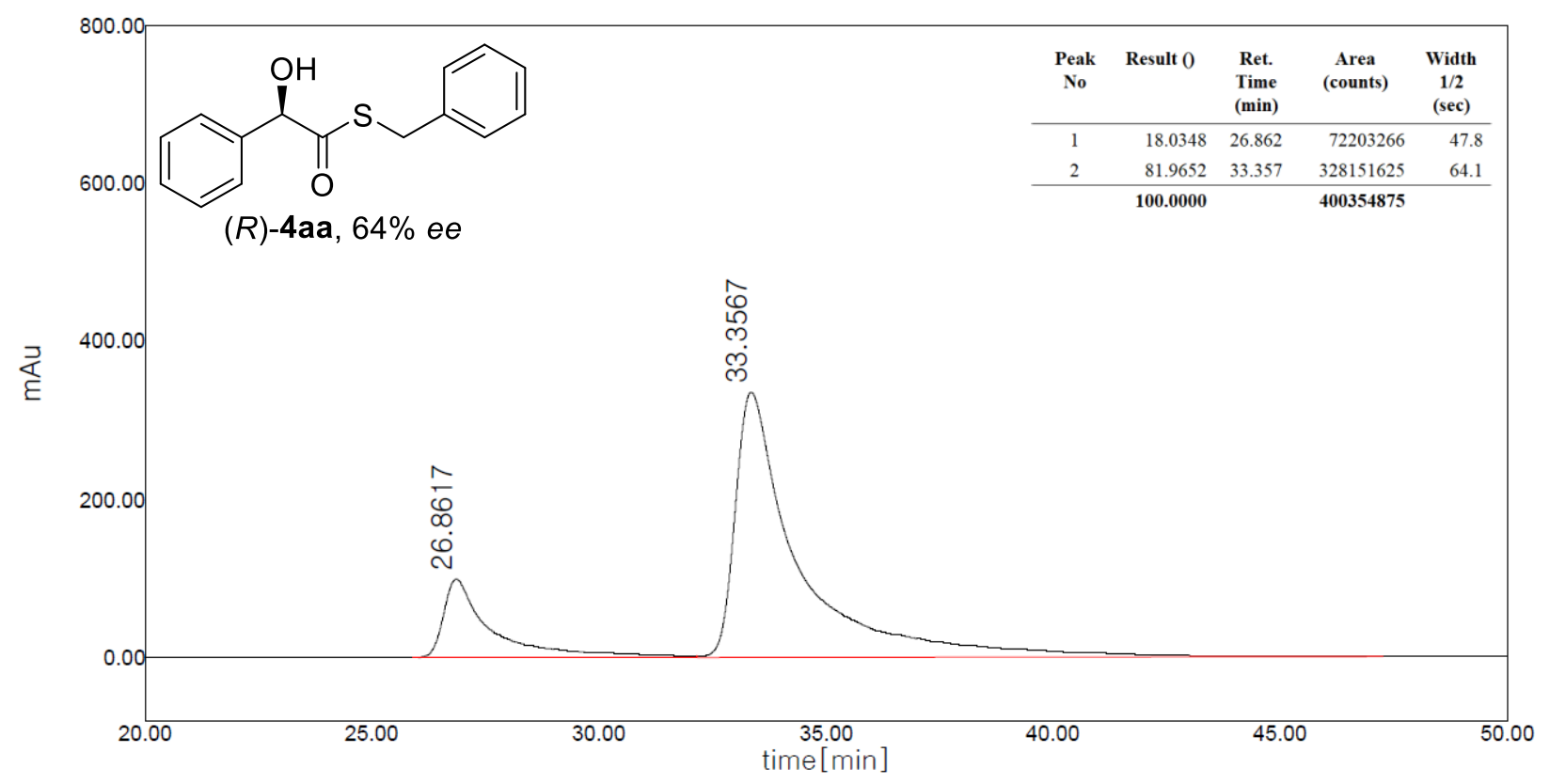

HPLC trace of 4aa (in CPME/THF)

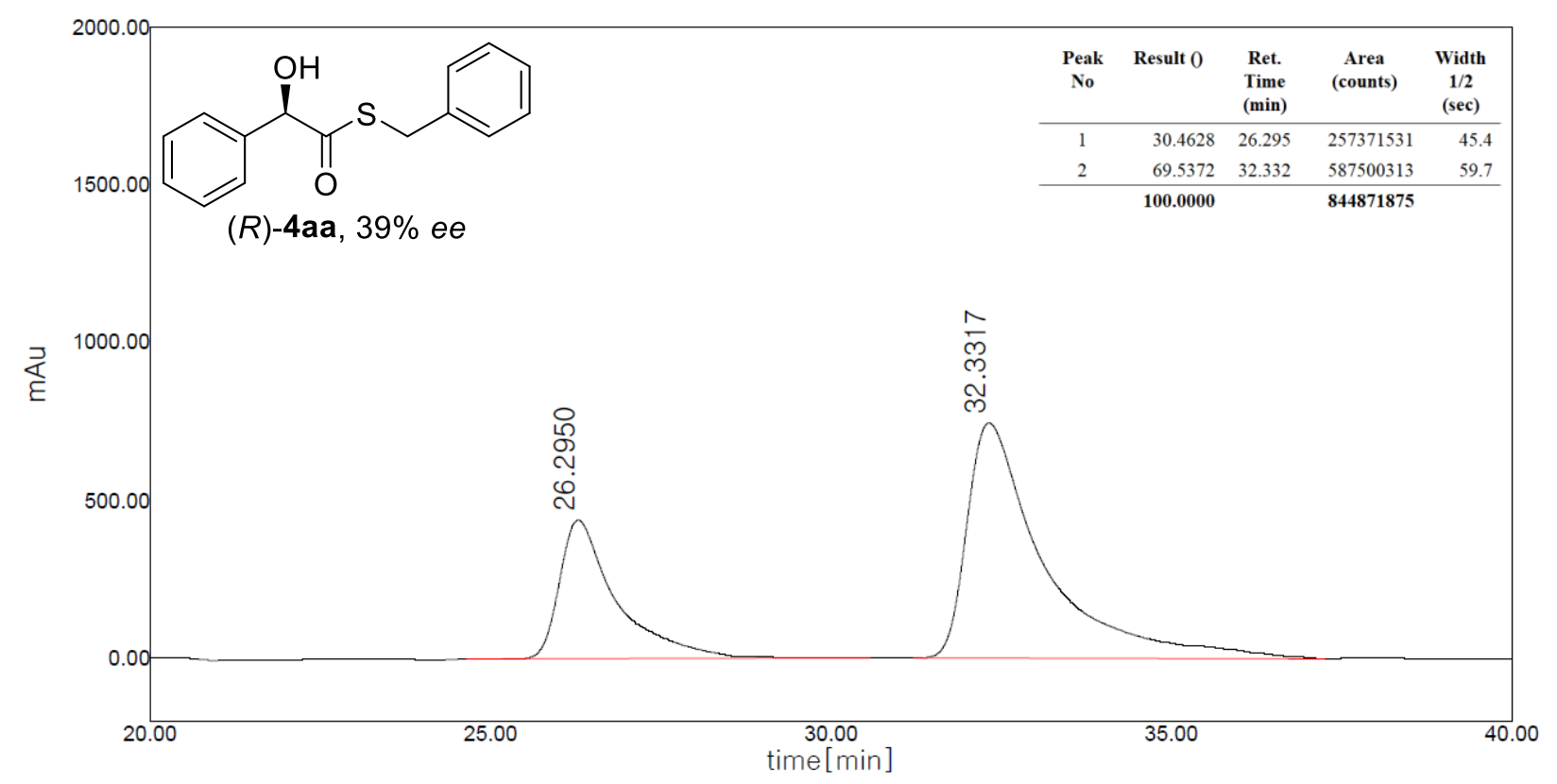

HPLC trace of 4aa (in 1,2-dimethoxy ethane) 


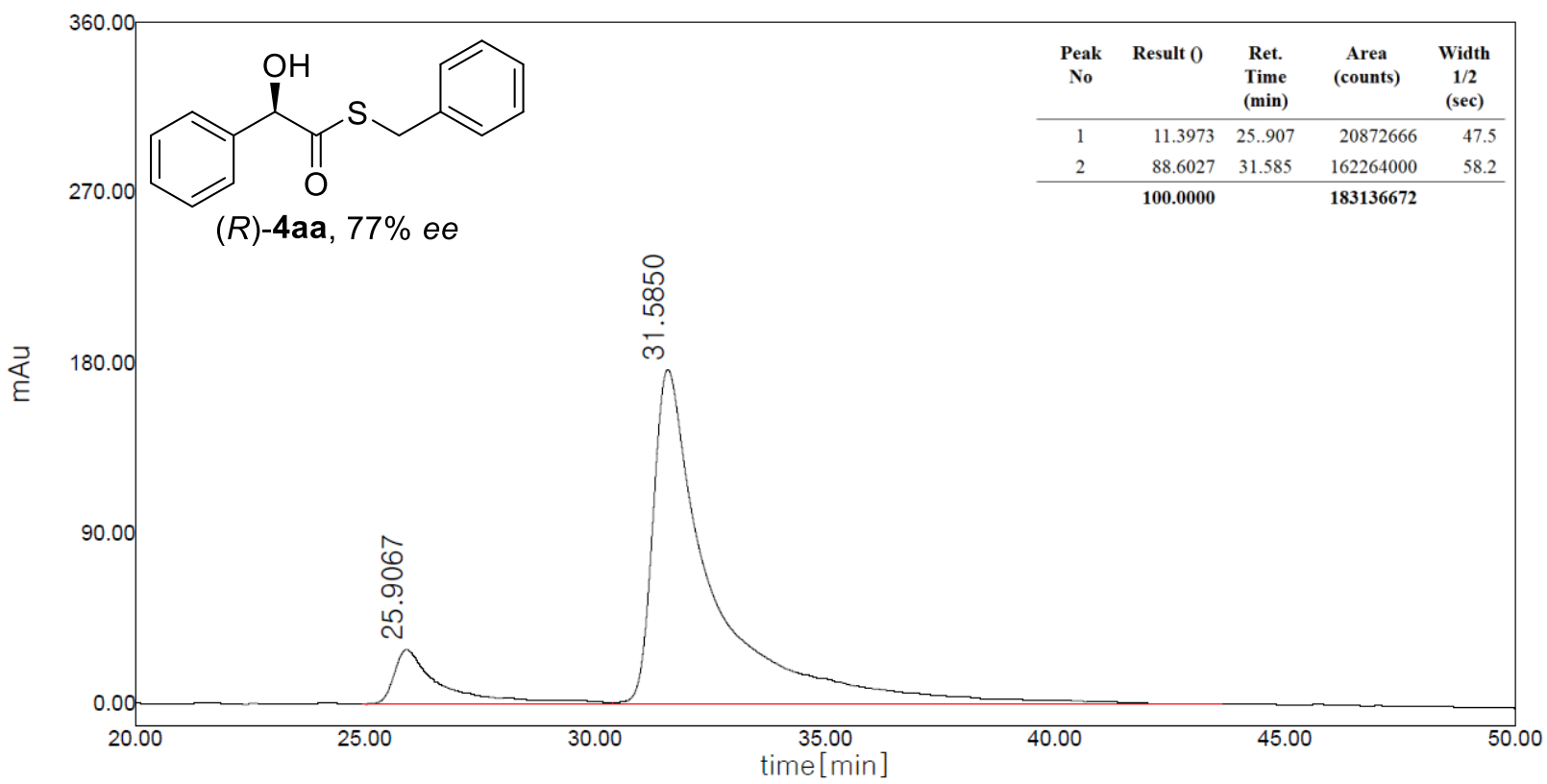

HPLC trace of $4 \mathbf{a a}$ (in eucalyptol)

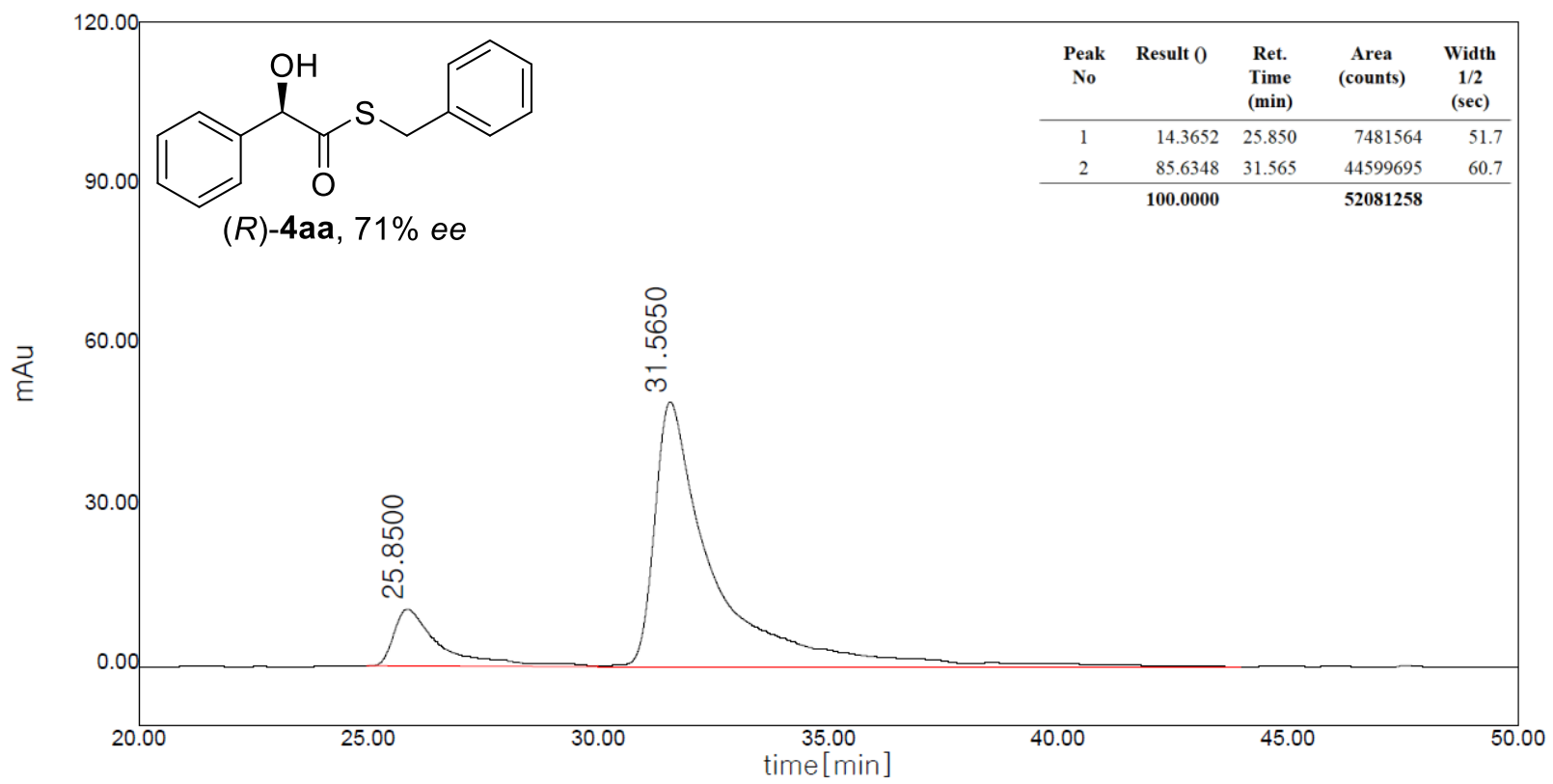

HPLC trace of 4aa (in (R)-(+)-limonene) 


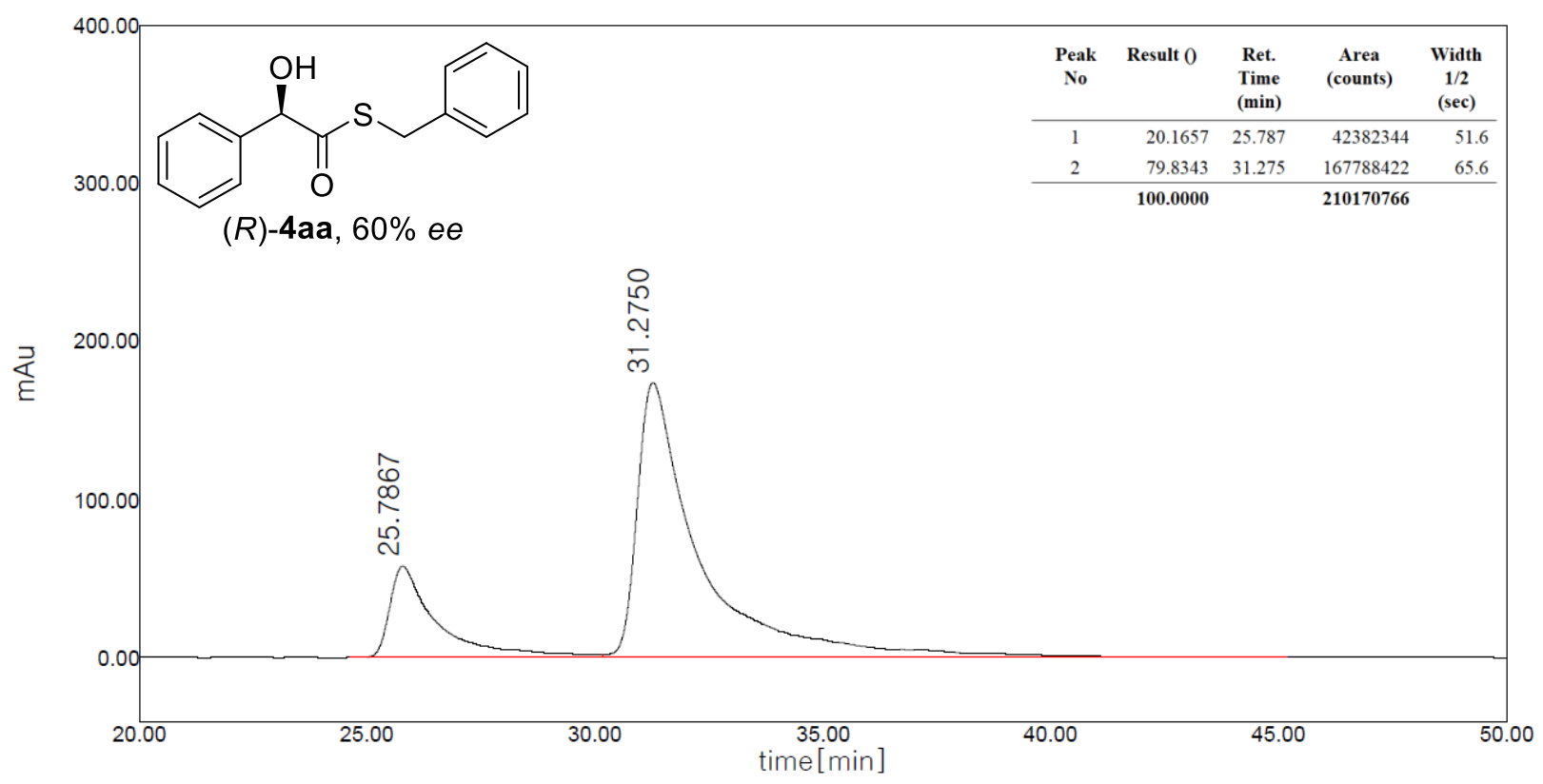

HPLC trace of $4 \mathbf{a a}$ (in (S)-(-)-limonene)

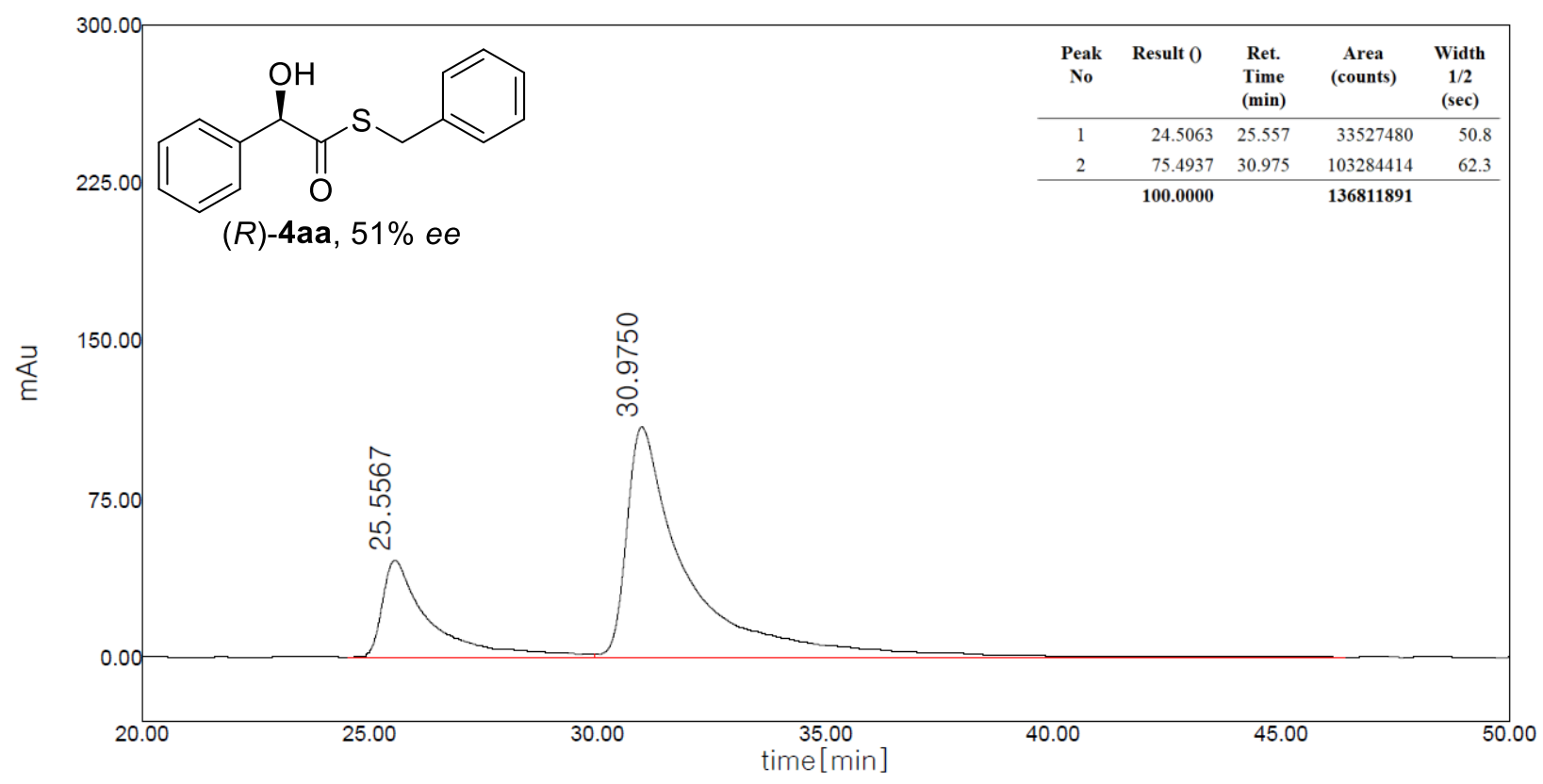

HPLC trace of $\mathbf{4 a a}($ in $(+)-(\alpha)$-pinene) 


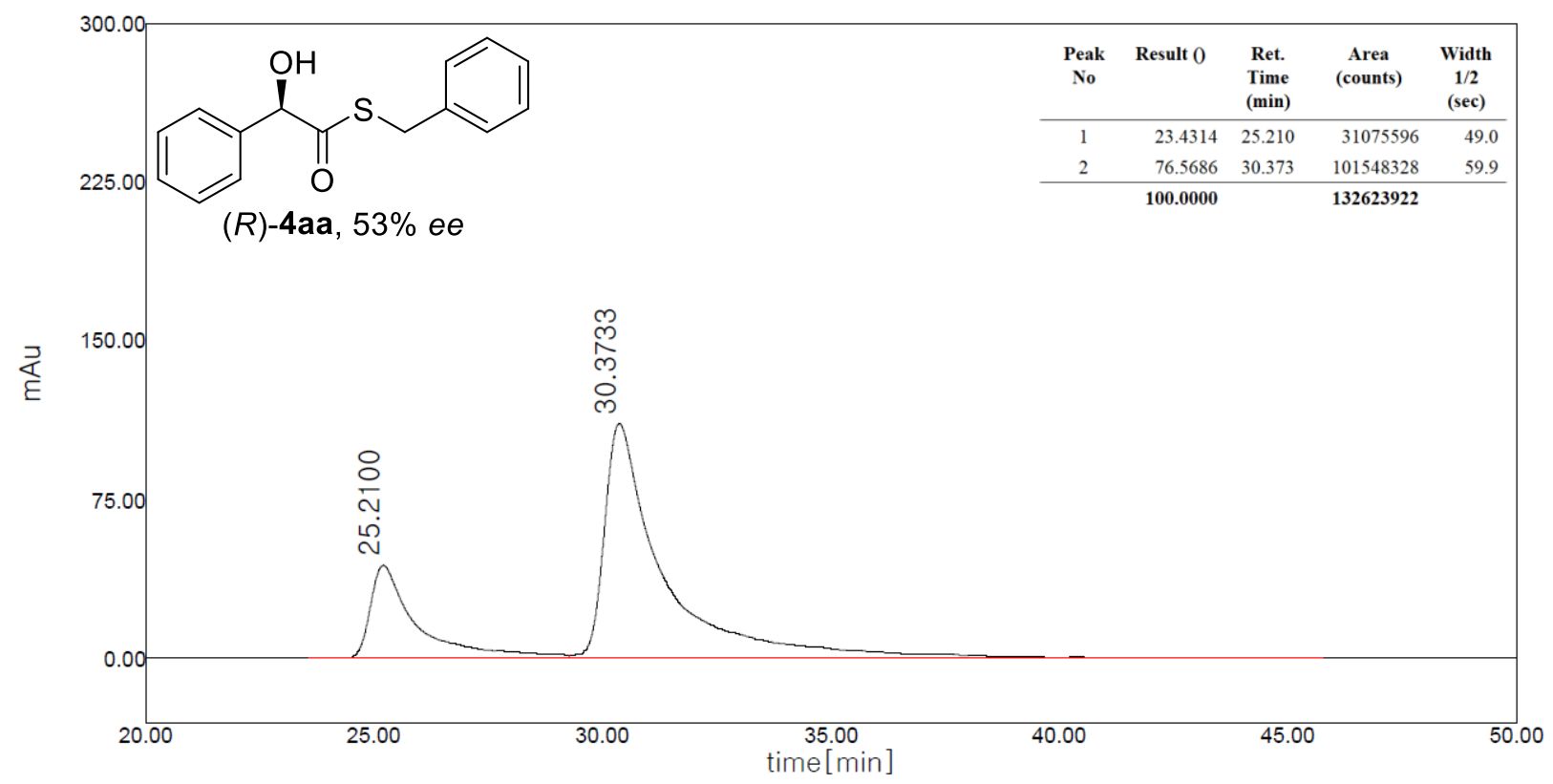

HPLC trace of 4aa (in (-)-( $\alpha)$-pinene) 


\section{HPLC traces of Table S2}

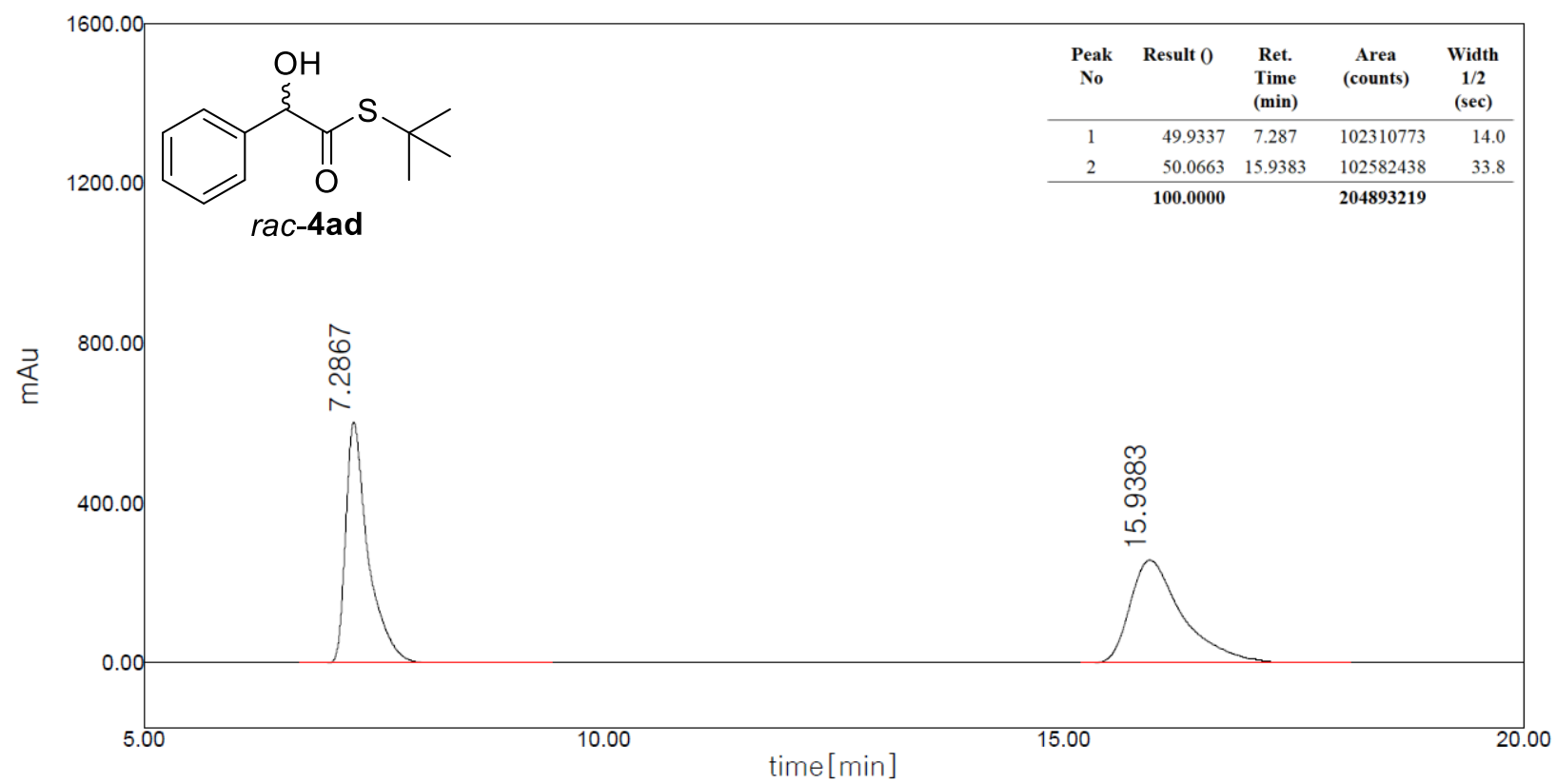

\section{HPLC trace of $r a c-4 a d$}

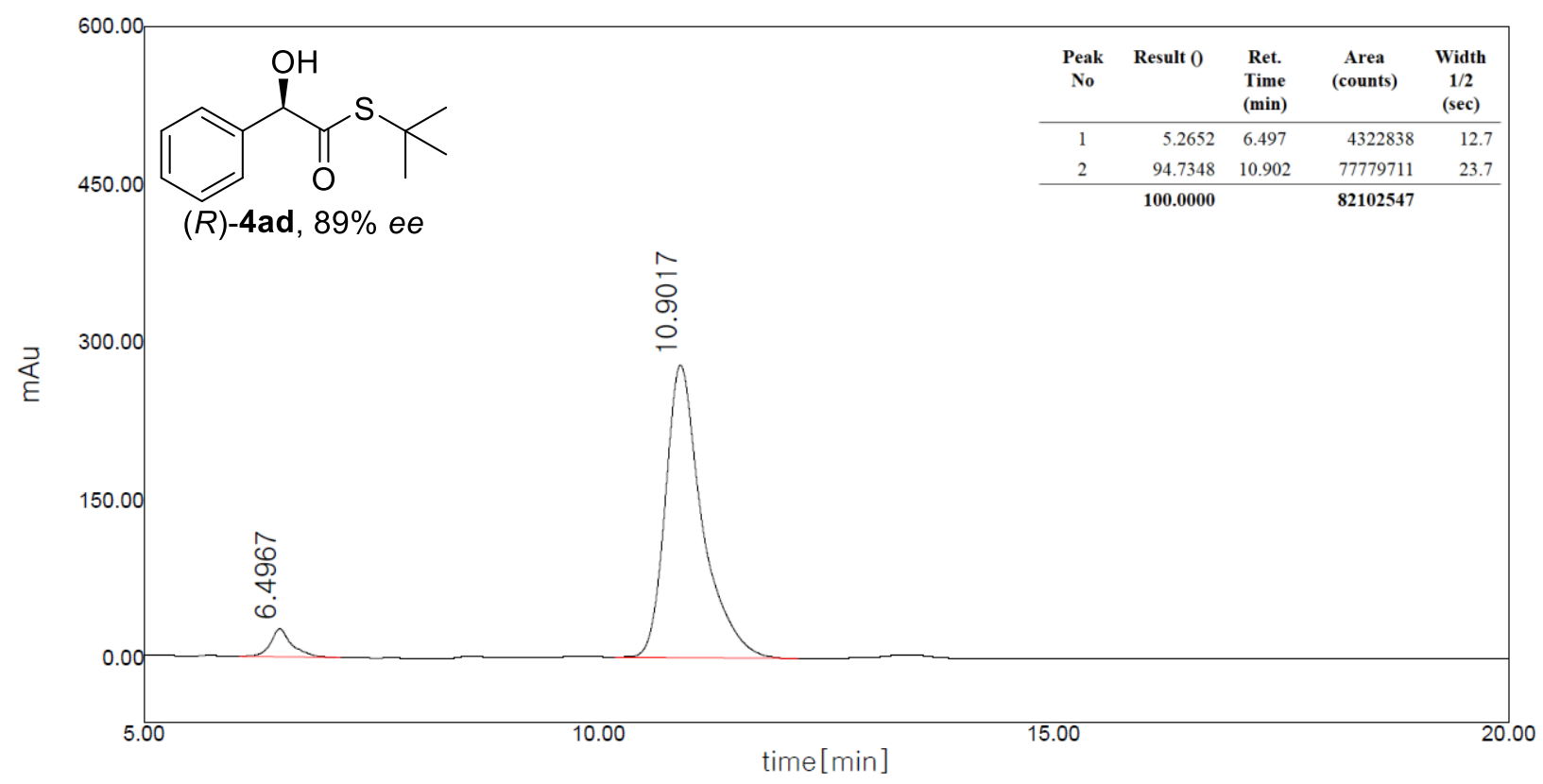

HPLC trace of 4ad (in $t$-BuSH ( 2 equiv)/Eucalyptol (5 equiv)) 


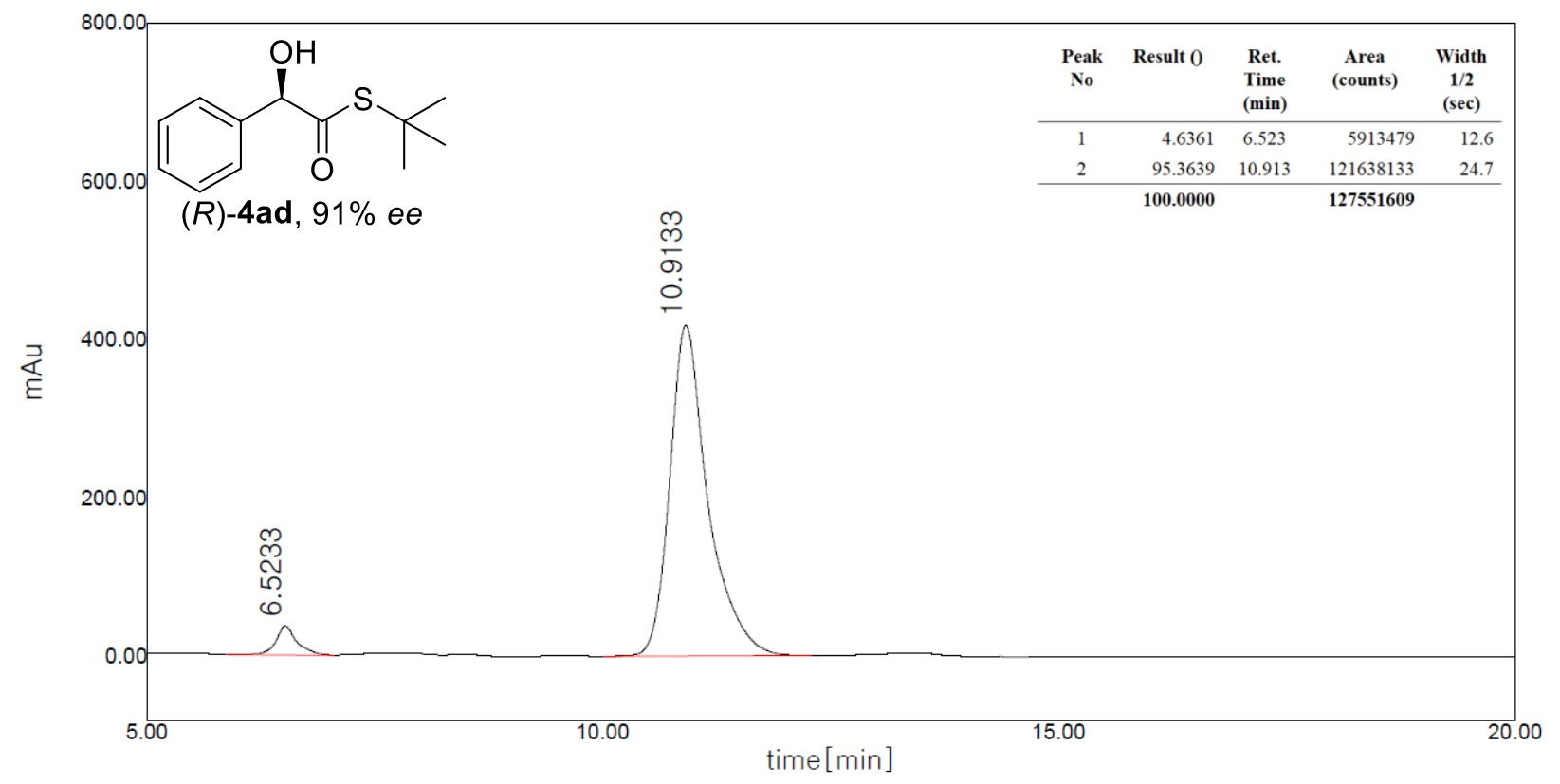

HPLC trace of 4ad (in $t$-BuSH (6 equiv)/Eucalyptol (5 equiv))

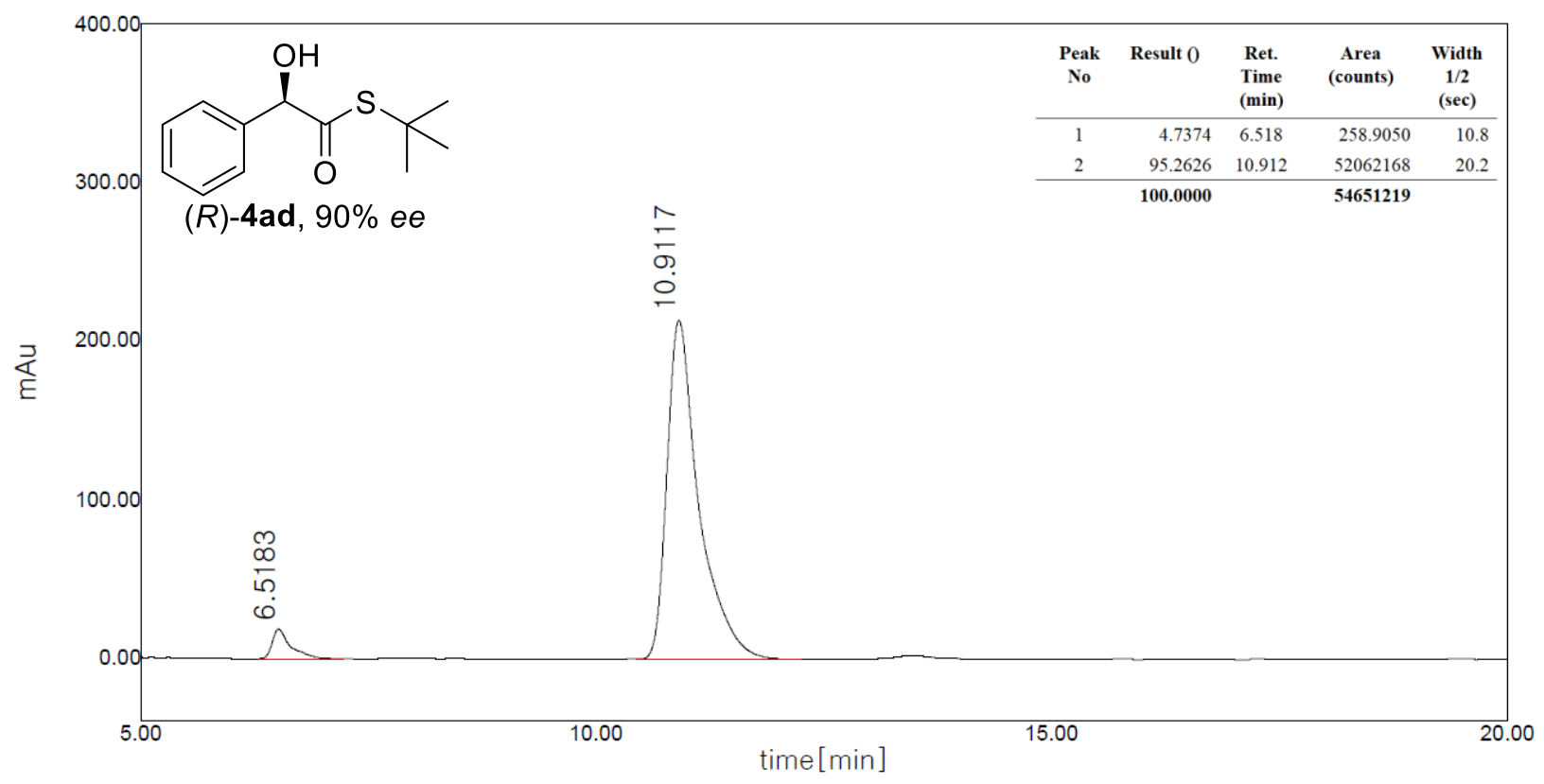

HPLC trace of 4ad (in $t$-BuSH (10 equiv)/Eucalyptol (5 equiv)) 


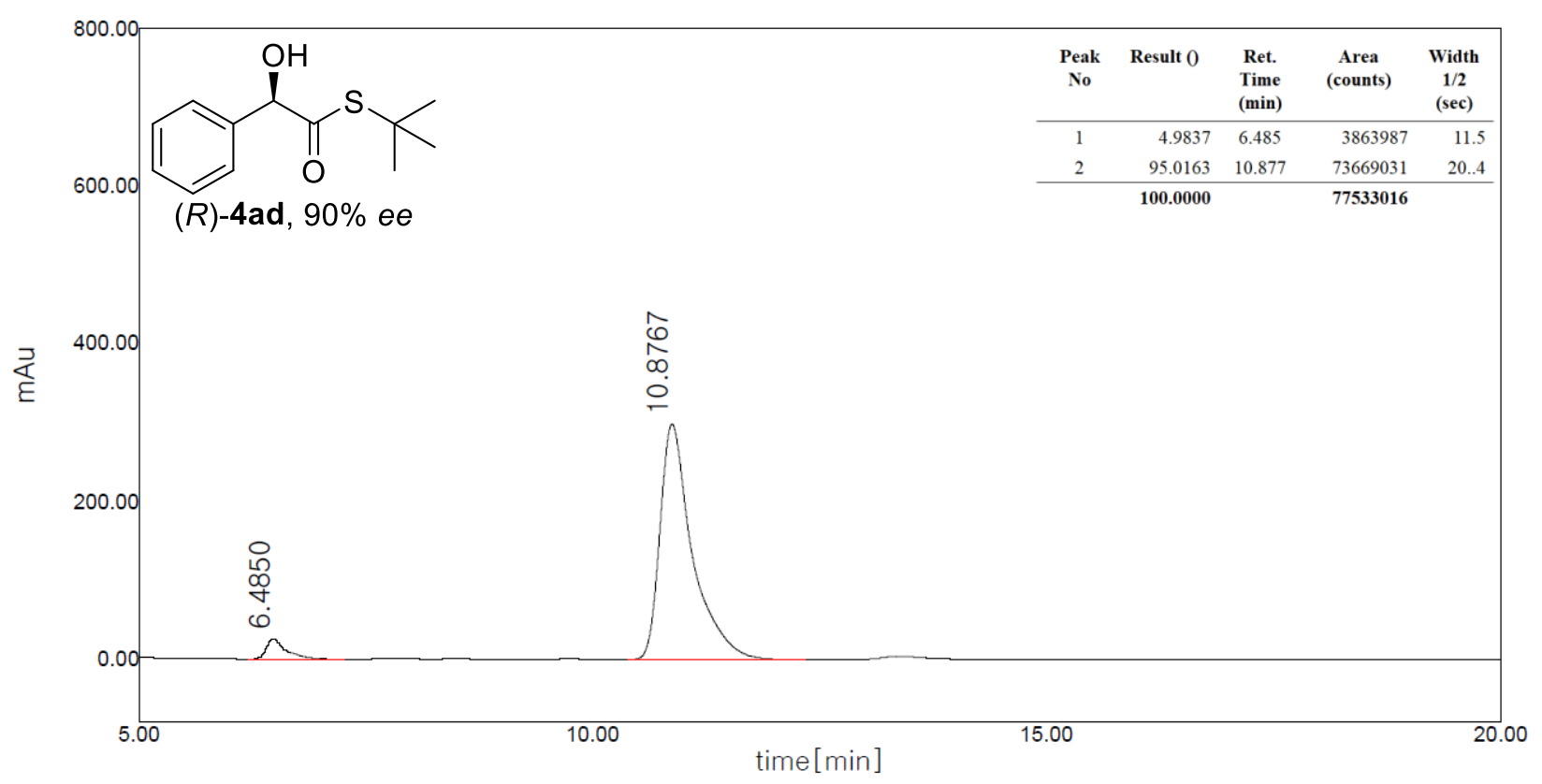

HPLC trace of 4ad (in $t$-BuSH (20 equiv)/Eucalyptol (5 equiv))

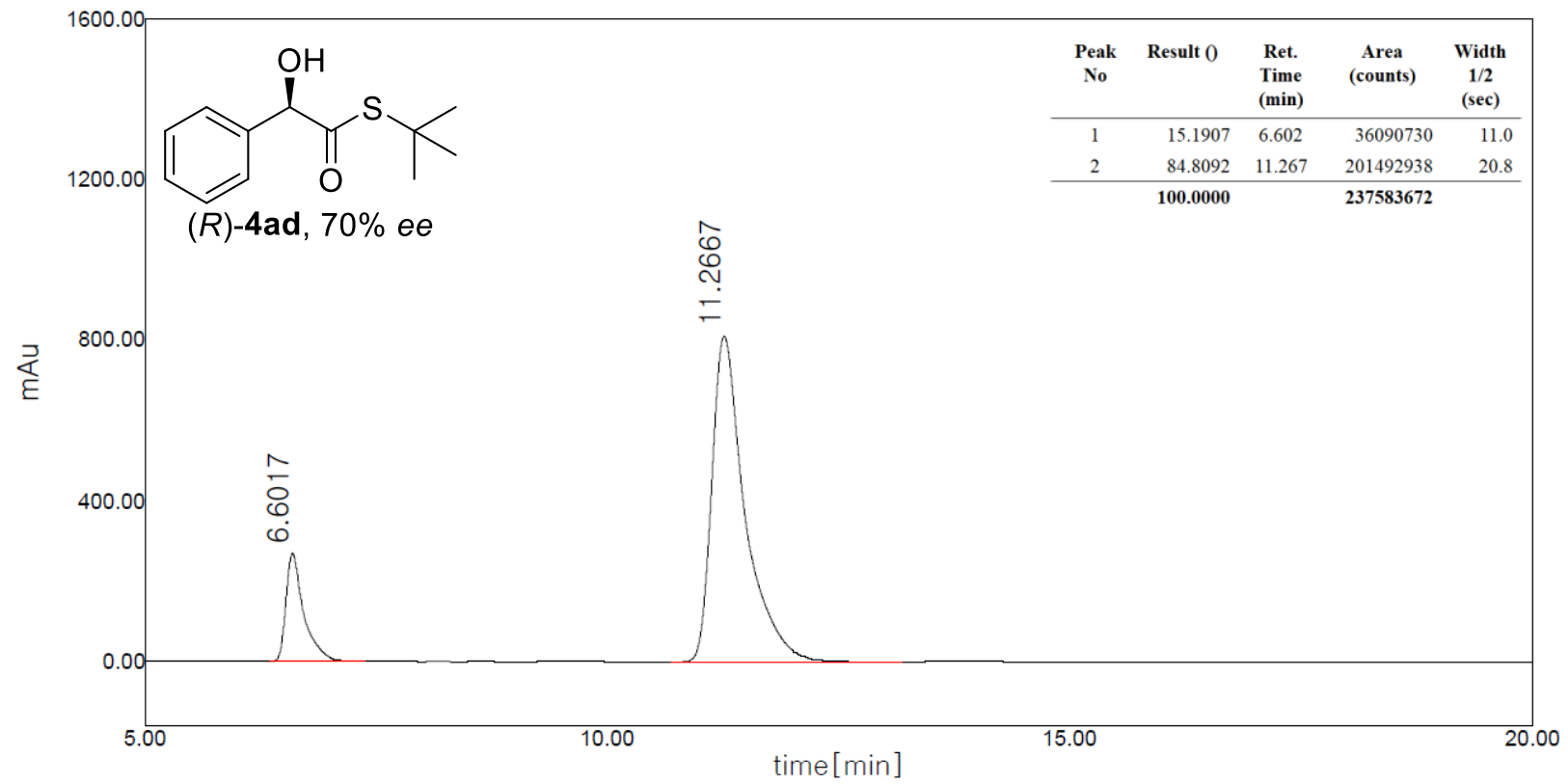

HPLC trace of 4 ad (in $t$-BuSH (6 equiv)) 


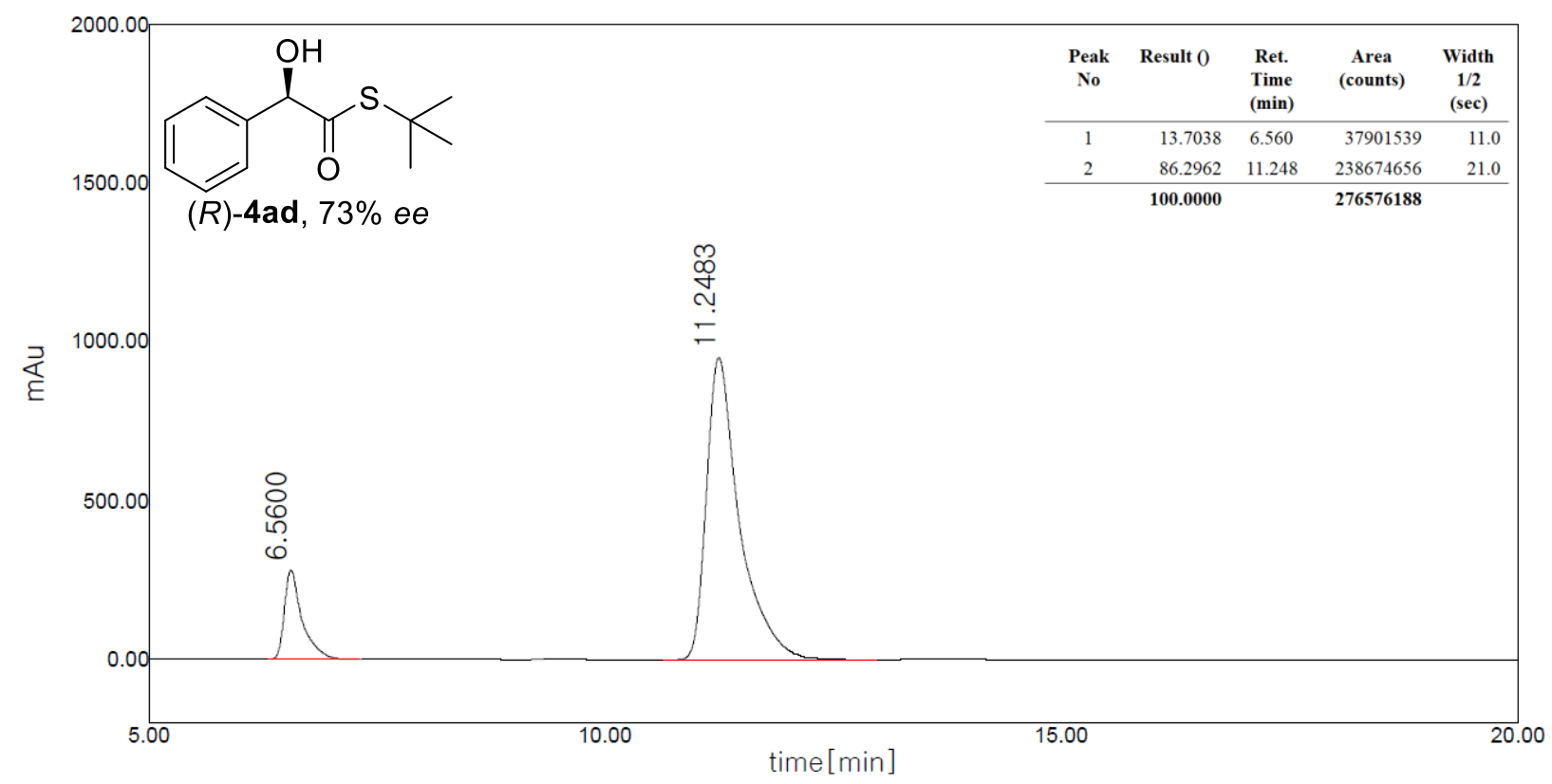

HPLC trace of 4ad (in $t$-BuSH (10 equiv))

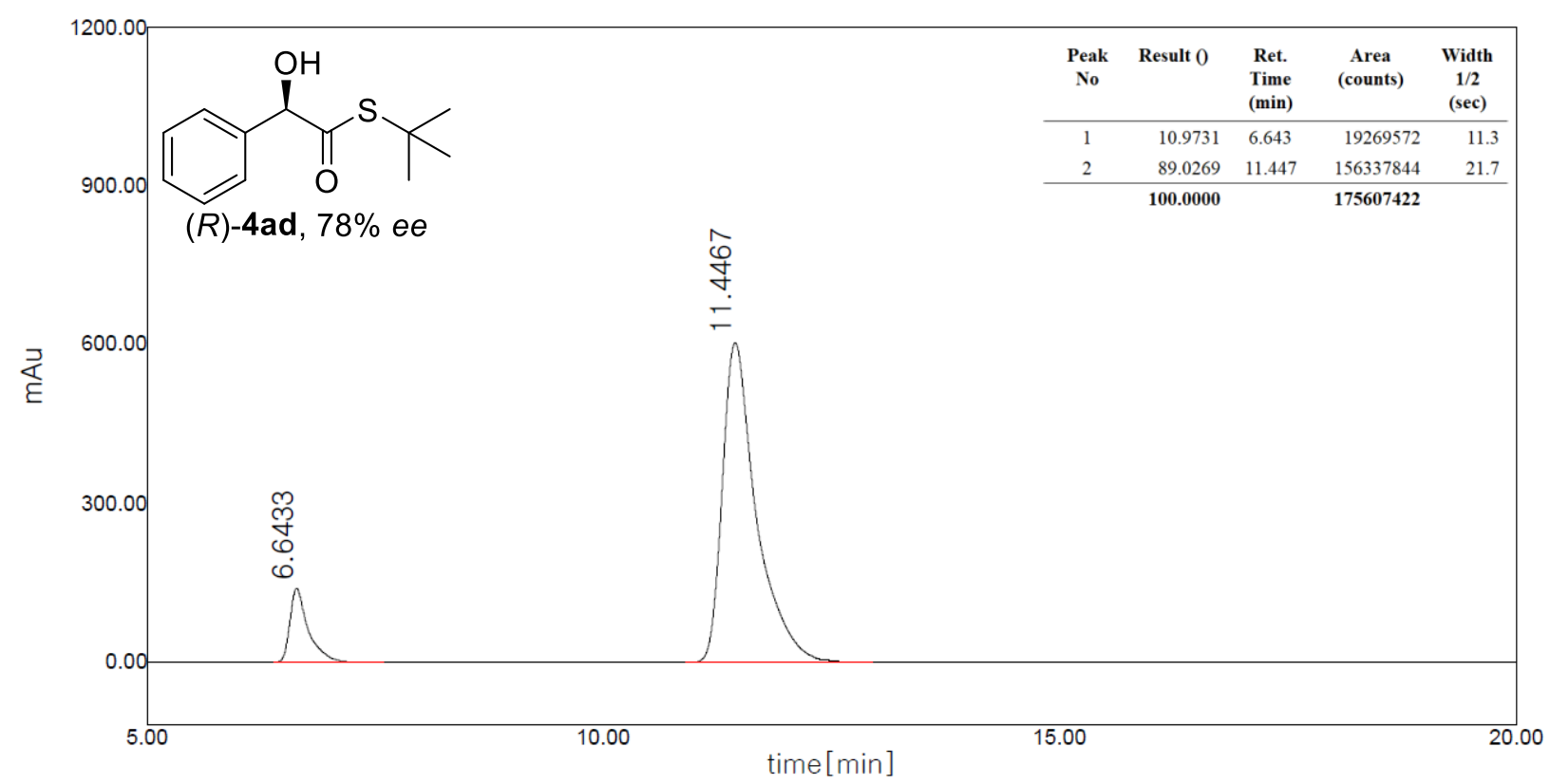

HPLC trace of $\mathbf{4 a d}$ (in $t$-BuSH (20 equiv)) 
HPLC trace of Scheme 1C/Figure S1

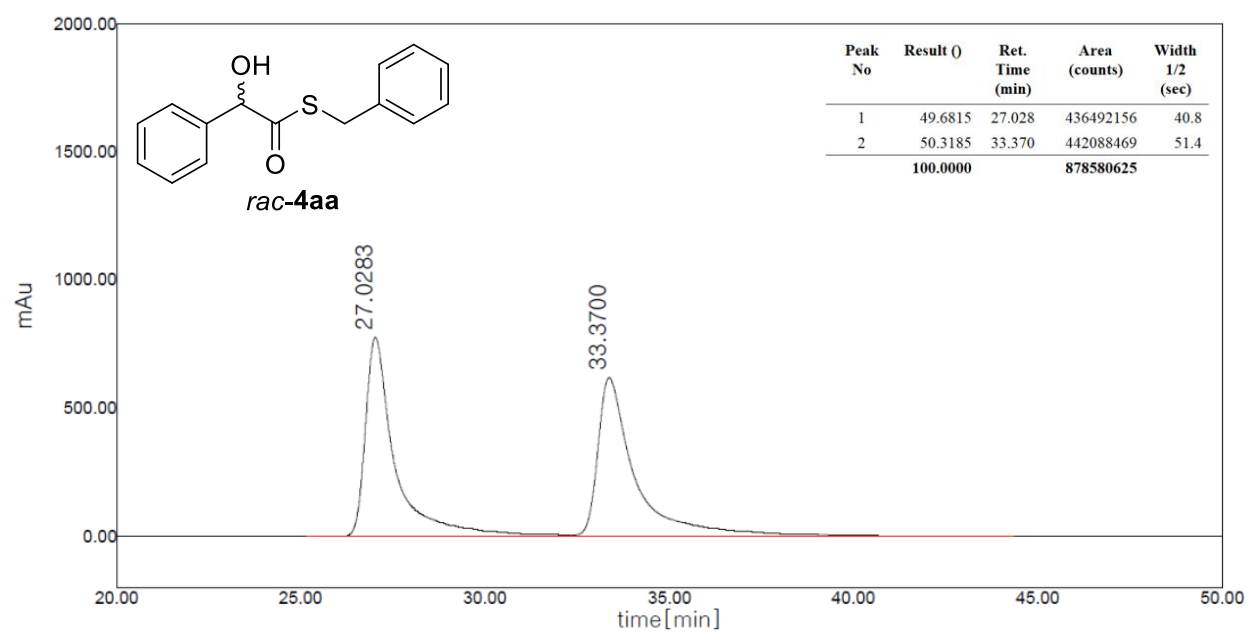

\section{HPLC trace of $r a c-\mathbf{4 a a}$}

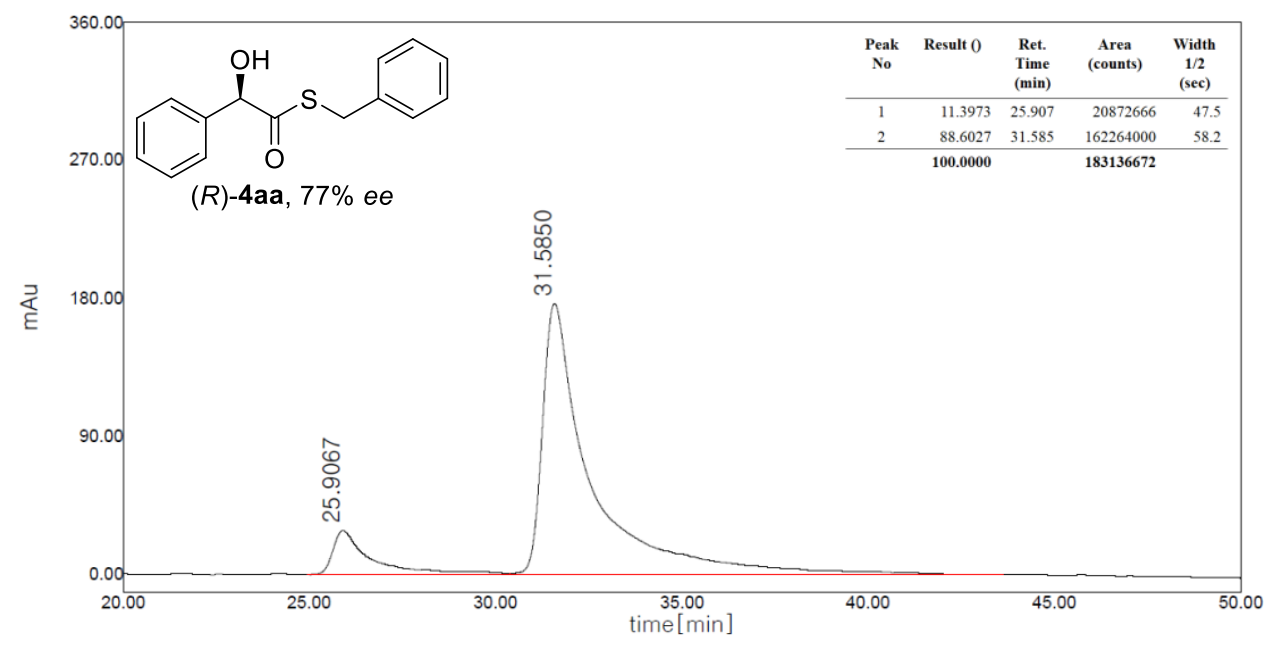

HPLC trace of 4aa-1.2 equiv. of 2a/5 equiv. of eucalyptol

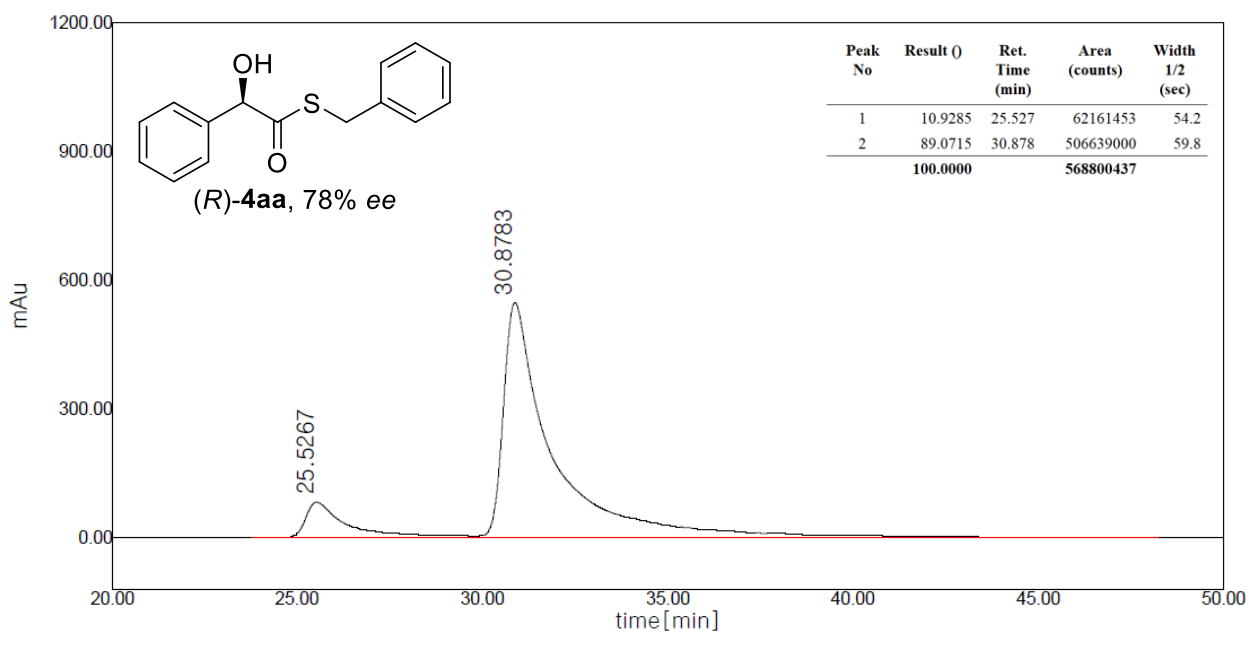

HPLC trace of 4aa-10 equiv. of $\mathbf{2 a} / 10$ equiv. of eucalyptol 


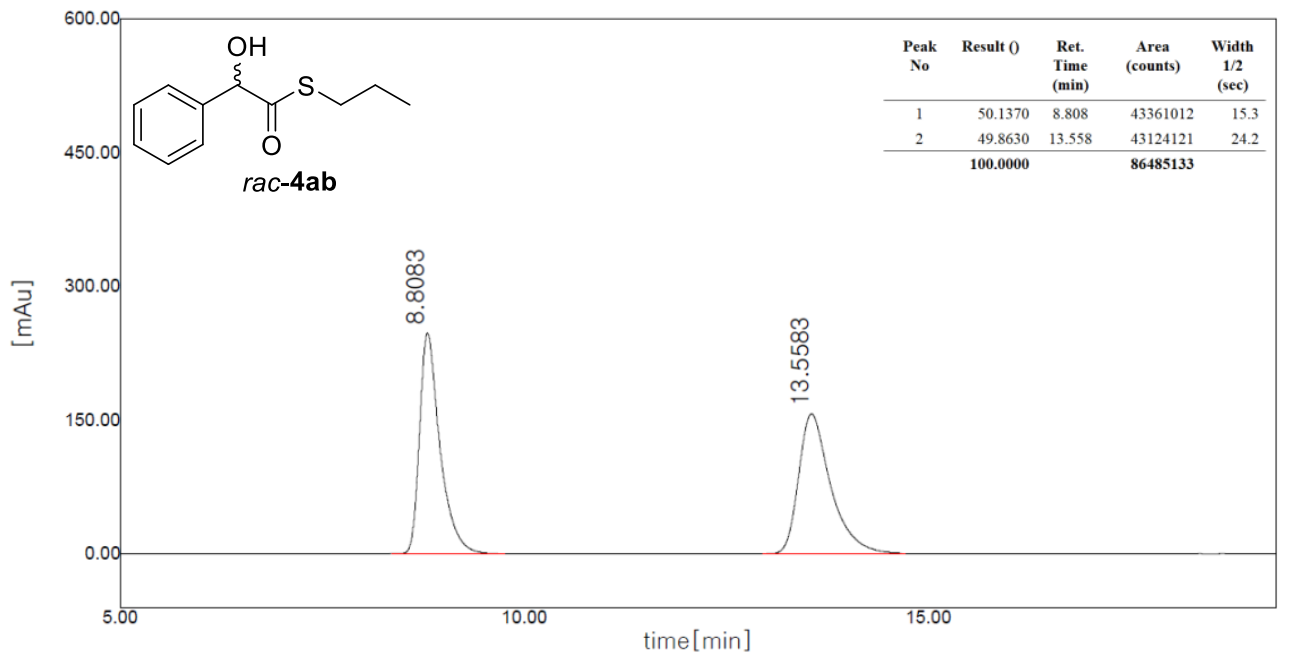

HPLC trace of $r a c-\mathbf{4 a b}$

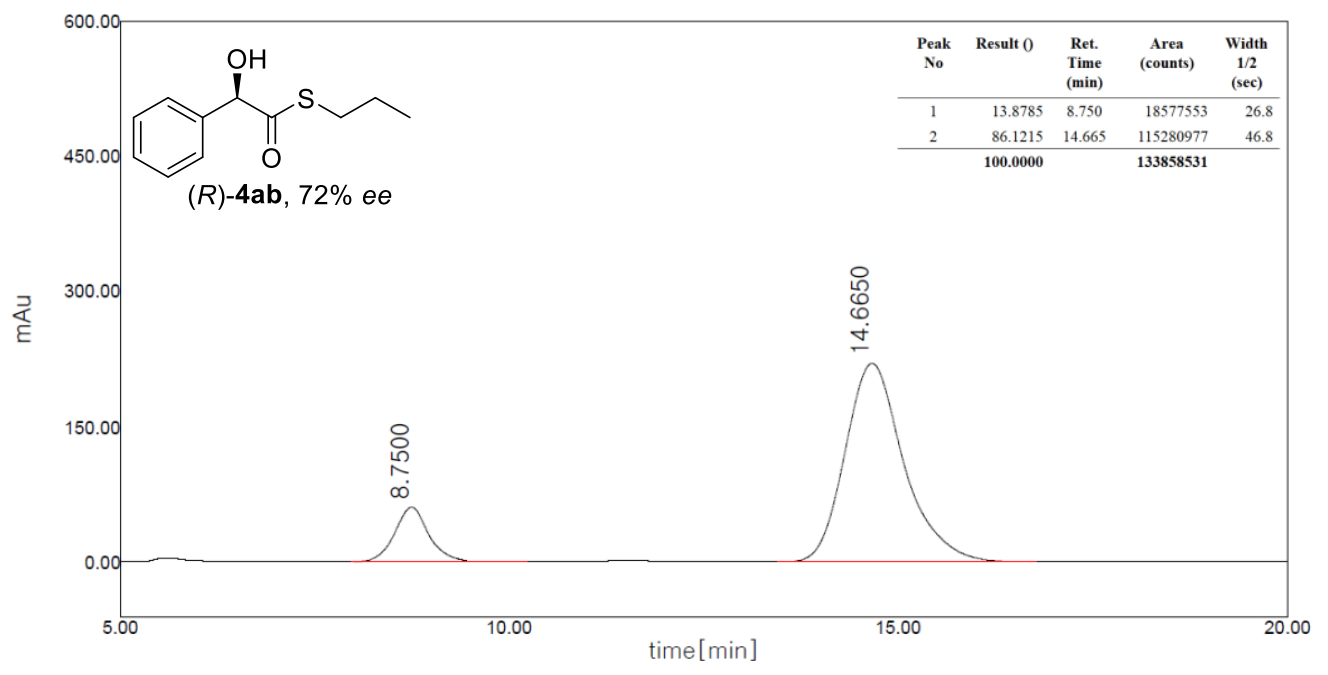

HPLC trace of $\mathbf{4 a b}-1.2$ equiv. of $\mathbf{2} \mathbf{a} / 5$ equiv. of eucalyptol

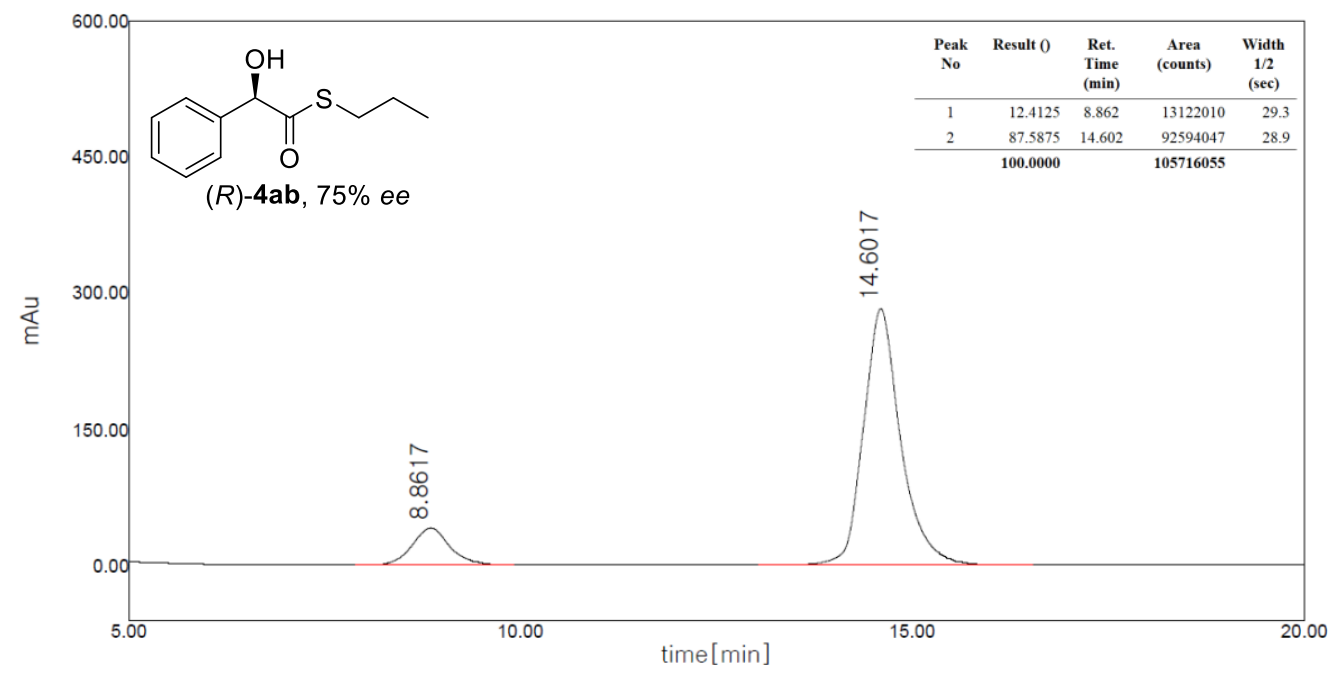

HPLC trace of 4ab-10 equiv. of $\mathbf{2 a} / 10$ equiv. of eucalyptol 


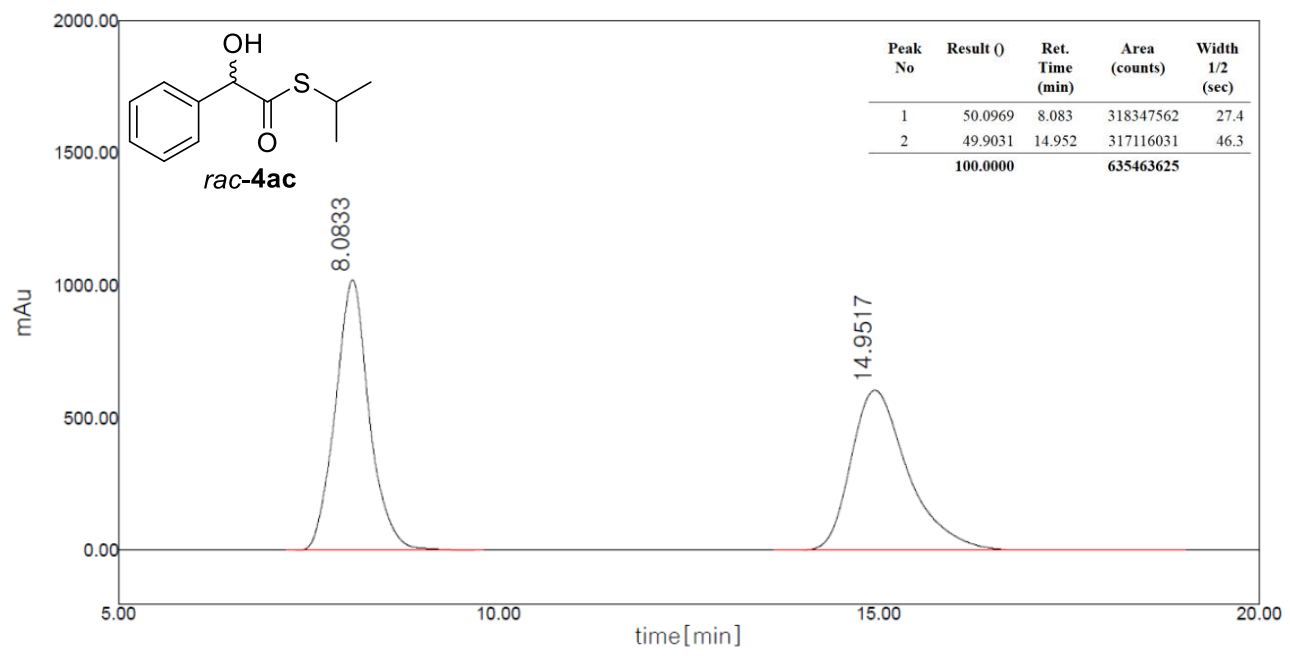

HPLC trace of rac-4ac

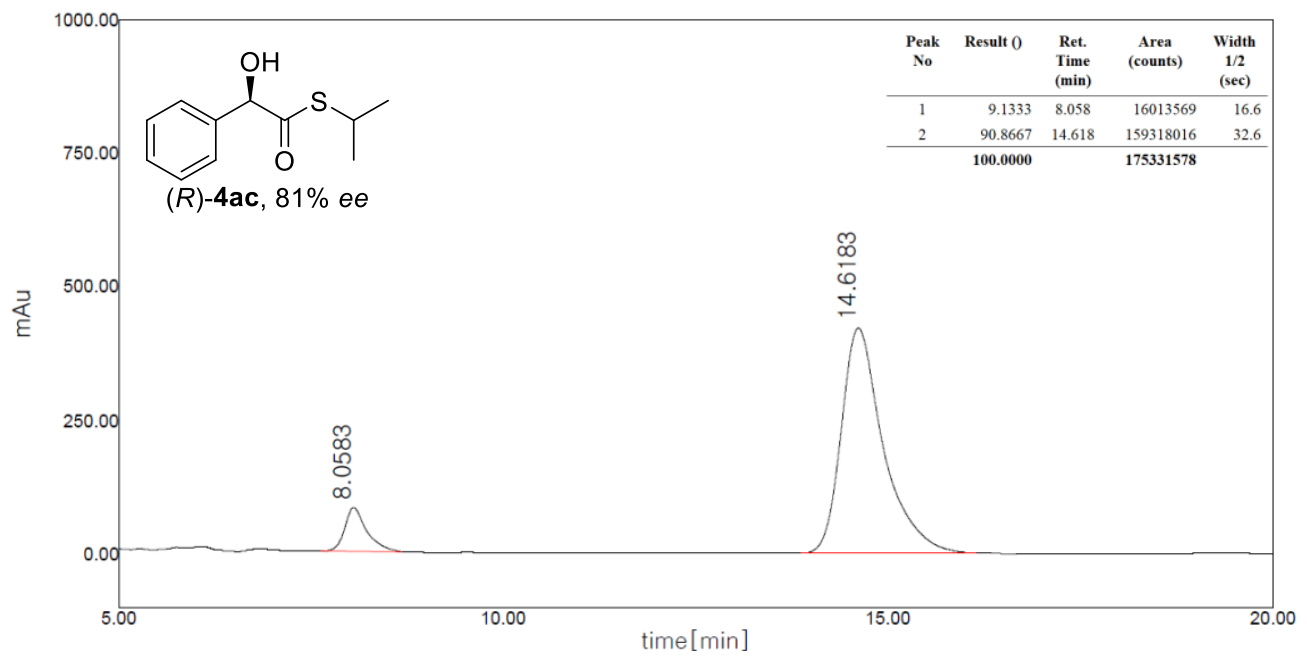

HPLC trace of $\mathbf{4 a c}-1.2$ equiv. of $\mathbf{2 a} / 5$ equiv. of eucalyptol

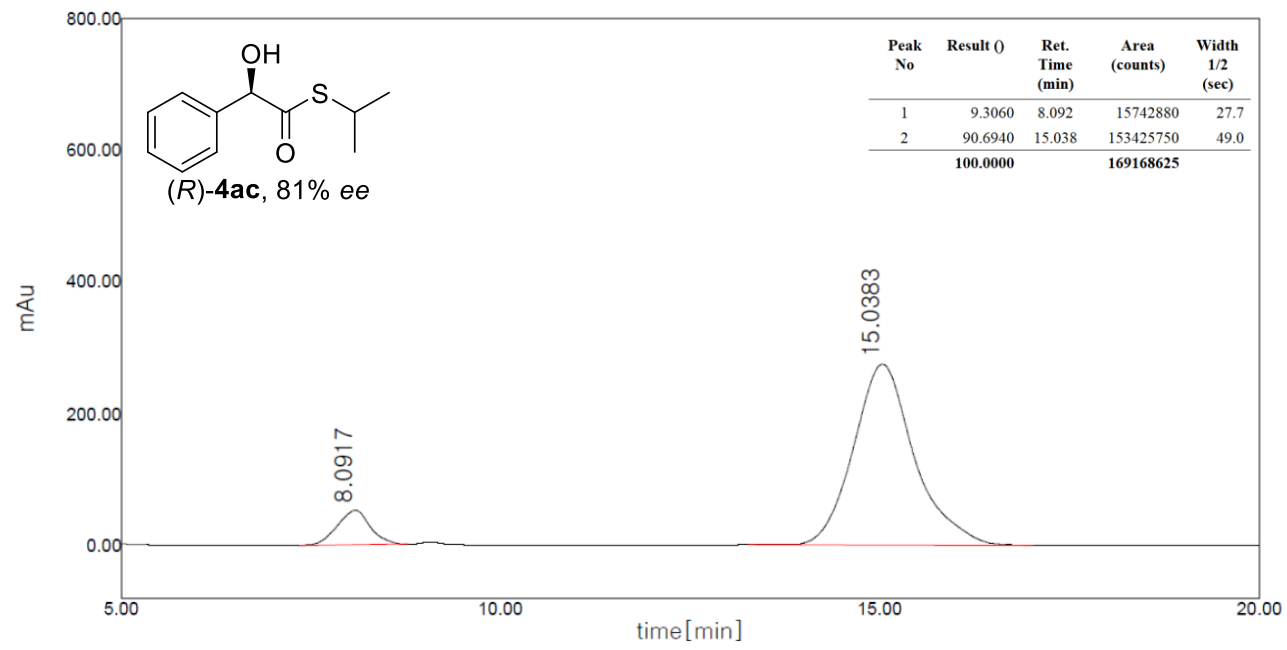

HPLC trace of 4ac-10 equiv. of $\mathbf{2 a / 1 0}$ equiv. of eucalyptol 


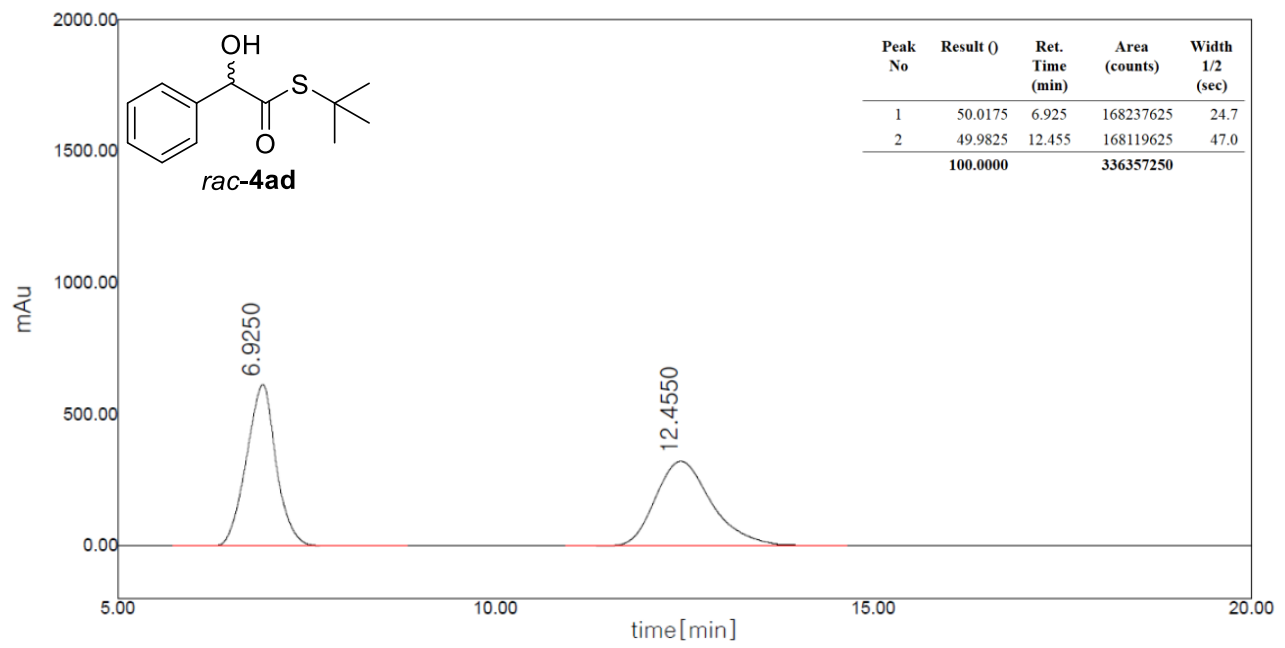

HPLC trace of rac-4ad

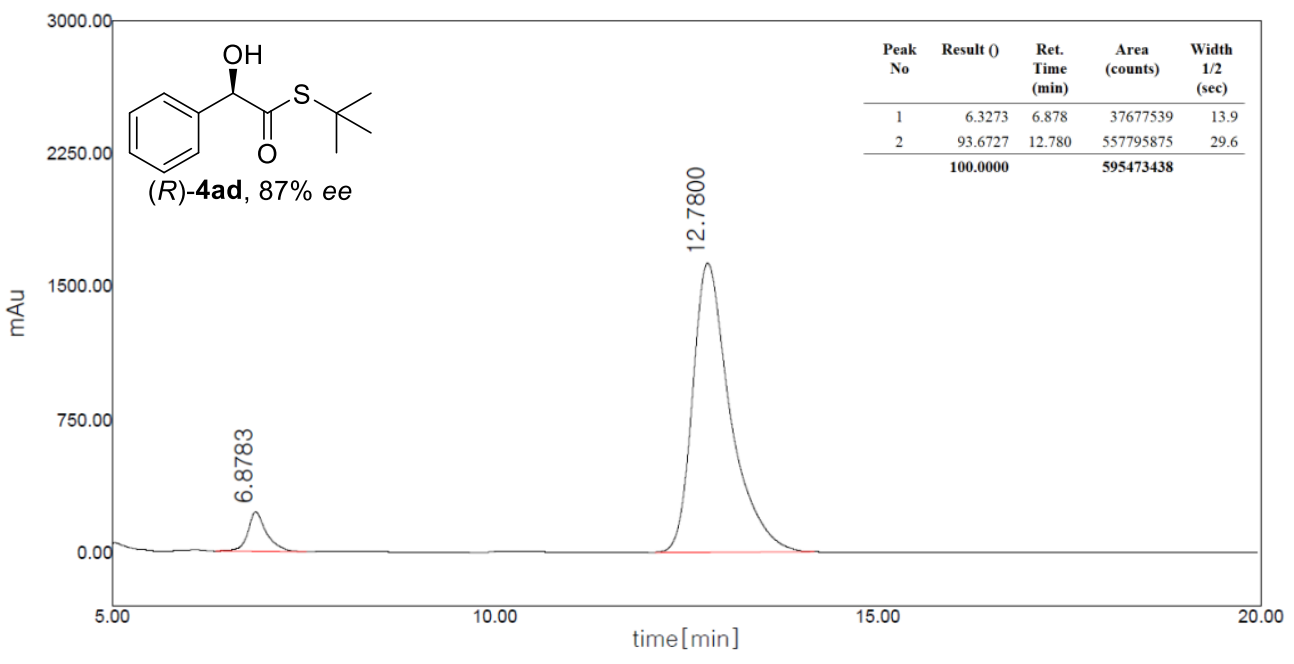

HPLC trace of $\mathbf{4 a d}-1.2$ equiv. of $\mathbf{2 a} / 5$ equiv. of eucalyptol

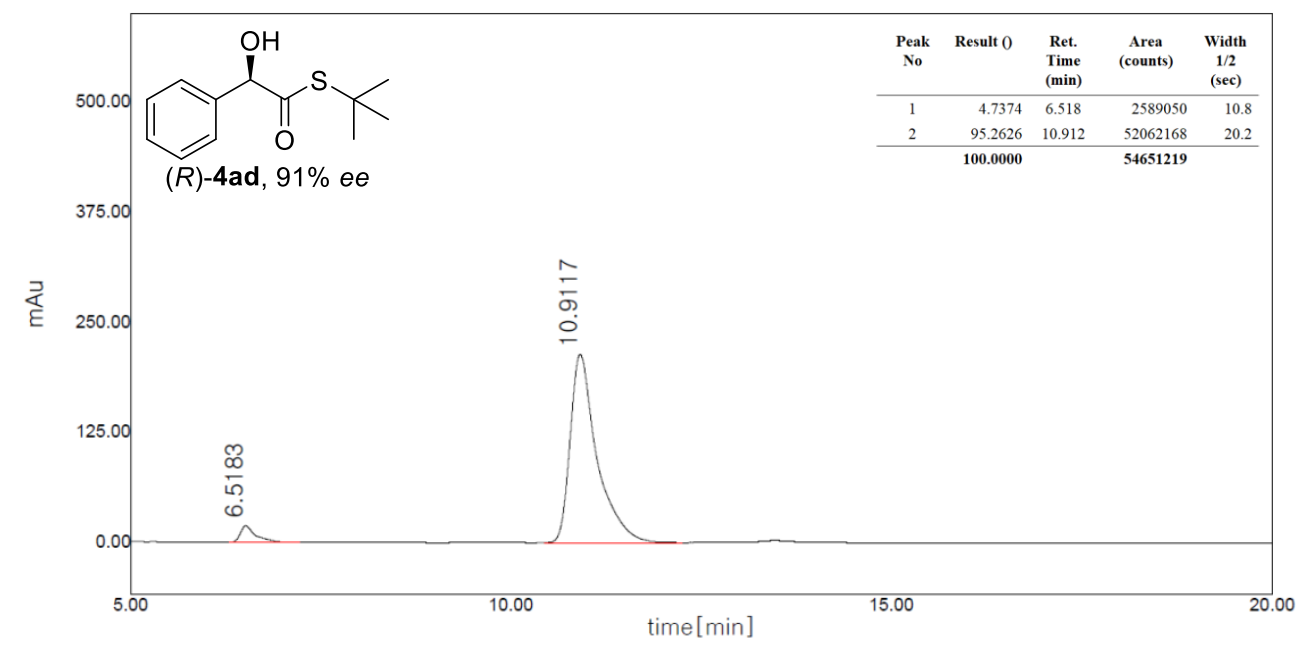

HPLC trace of 4ad-10 equiv. of $\mathbf{2 a / 1 0}$ equiv. of eucalyptol 


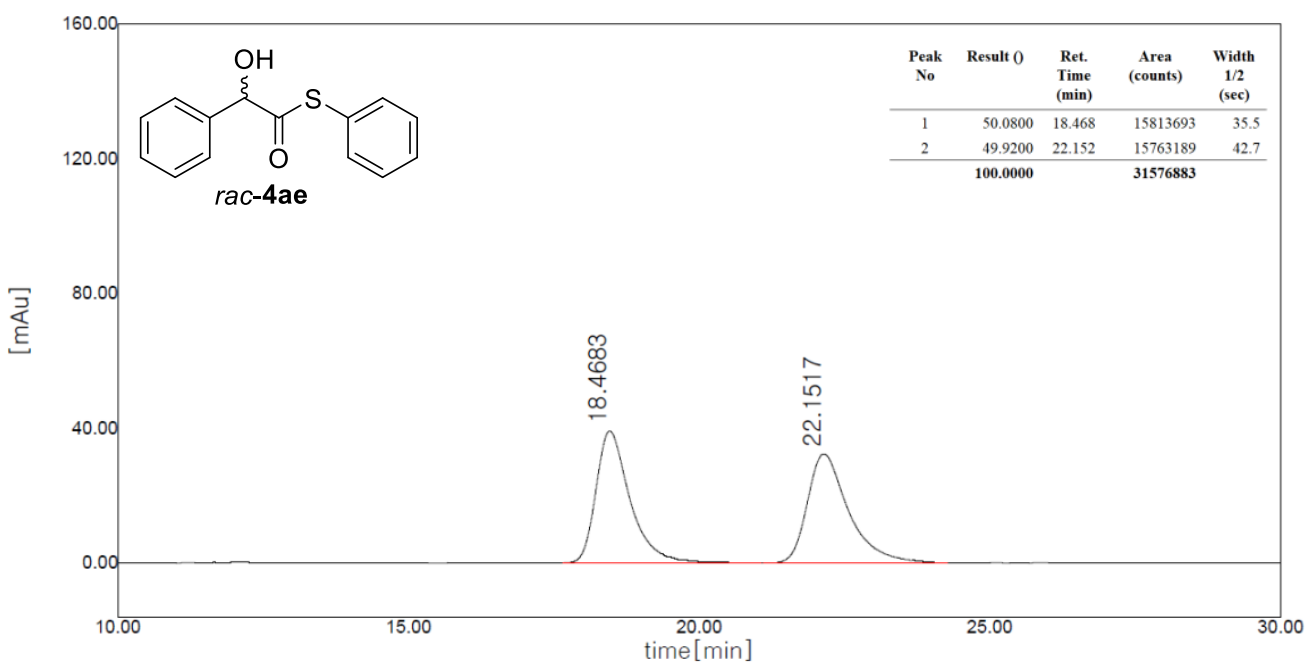

HPLC trace of $r a c-\mathbf{4 a e}$

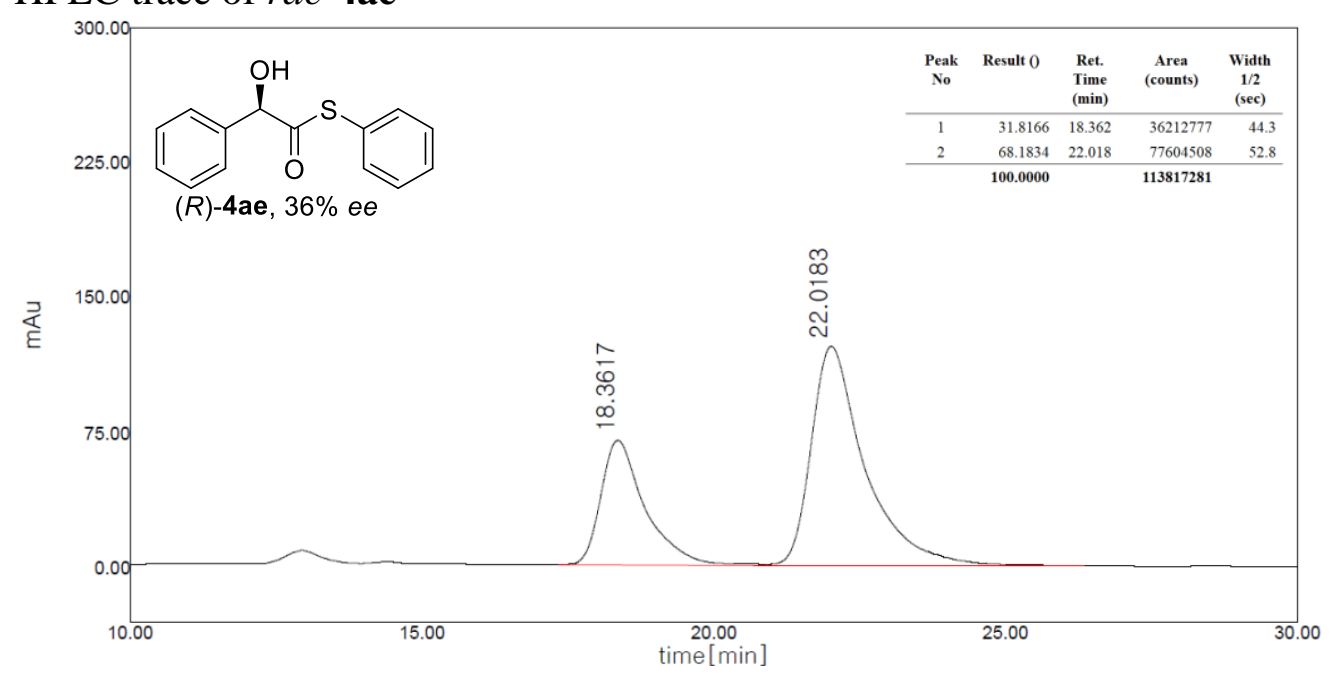

HPLC trace of $4 \mathbf{a e}-1.2$ equiv. of $\mathbf{2 a} / 5$ equiv. of eucalyptol

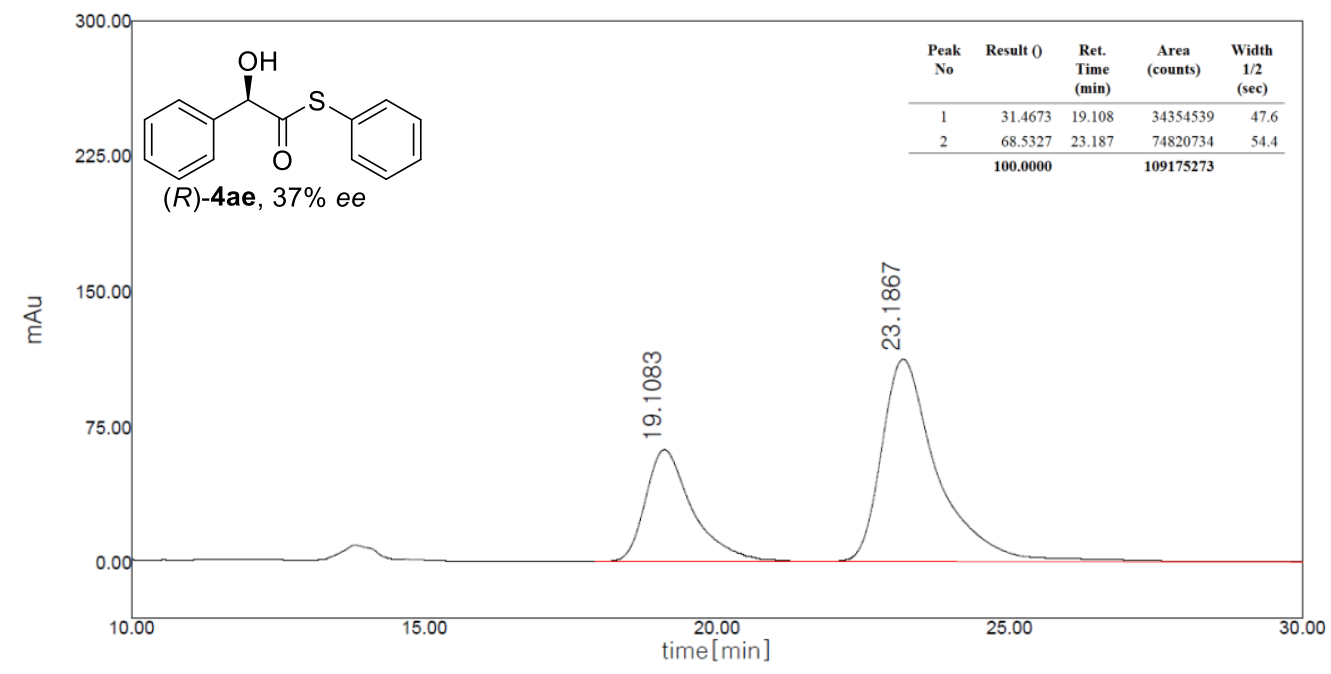

HPLC trace of 4ae-10 equiv. of $\mathbf{2 a} / 10$ equiv. of eucalyptol 


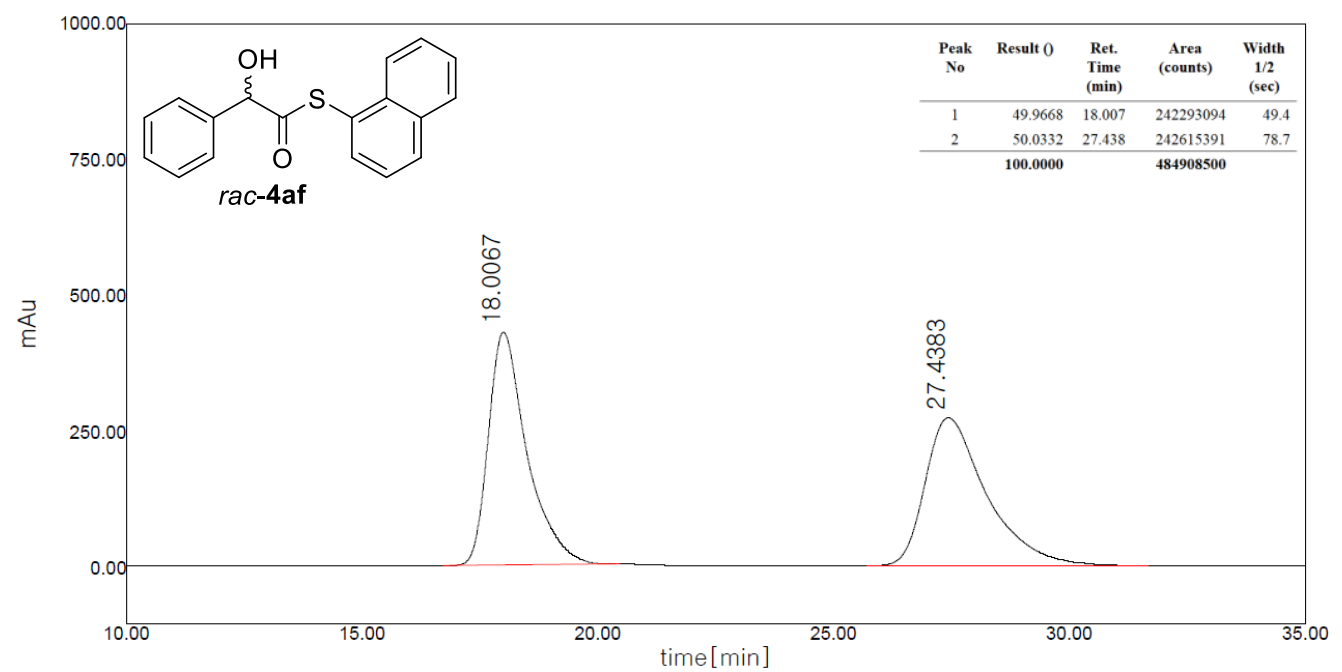

HPLC trace of rac-4af

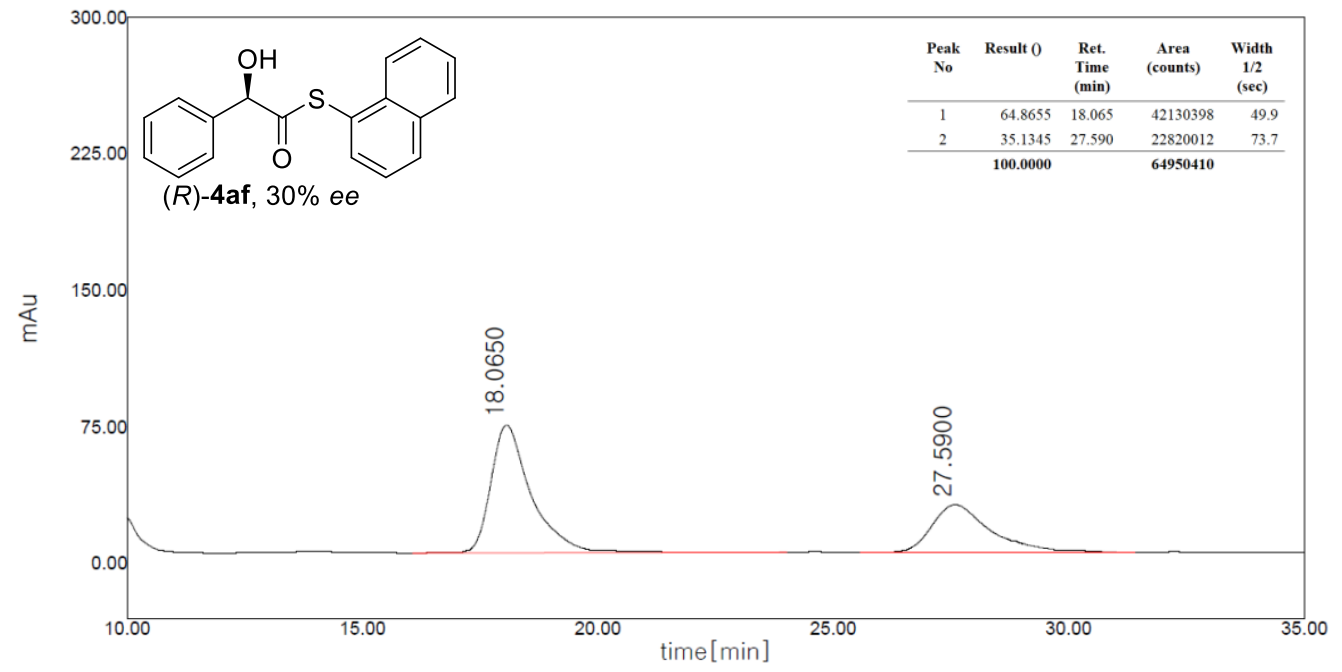

HPLC trace of 4af-1.2 equiv. of $\mathbf{2 a} / 5$ equiv. of eucalyptol

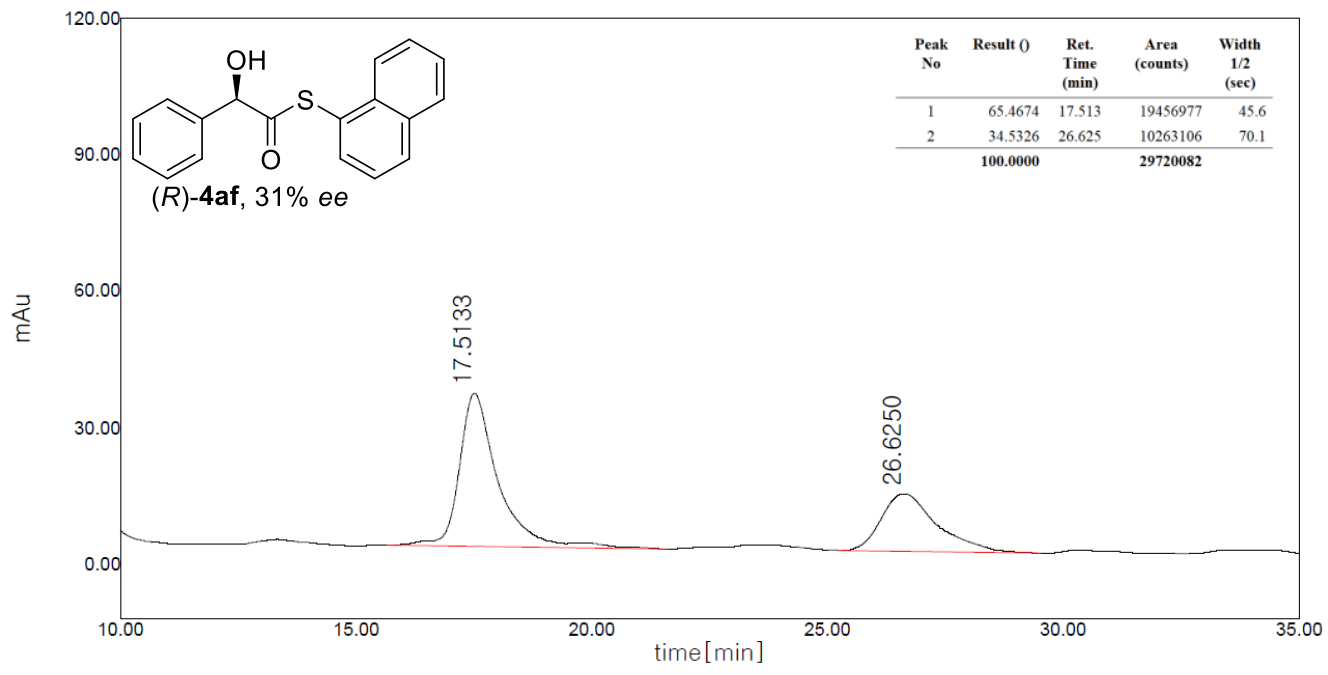

HPLC trace of $\mathbf{4 a f}-10$ equiv. of $\mathbf{2 a} / 10$ equiv. of eucalyptol 


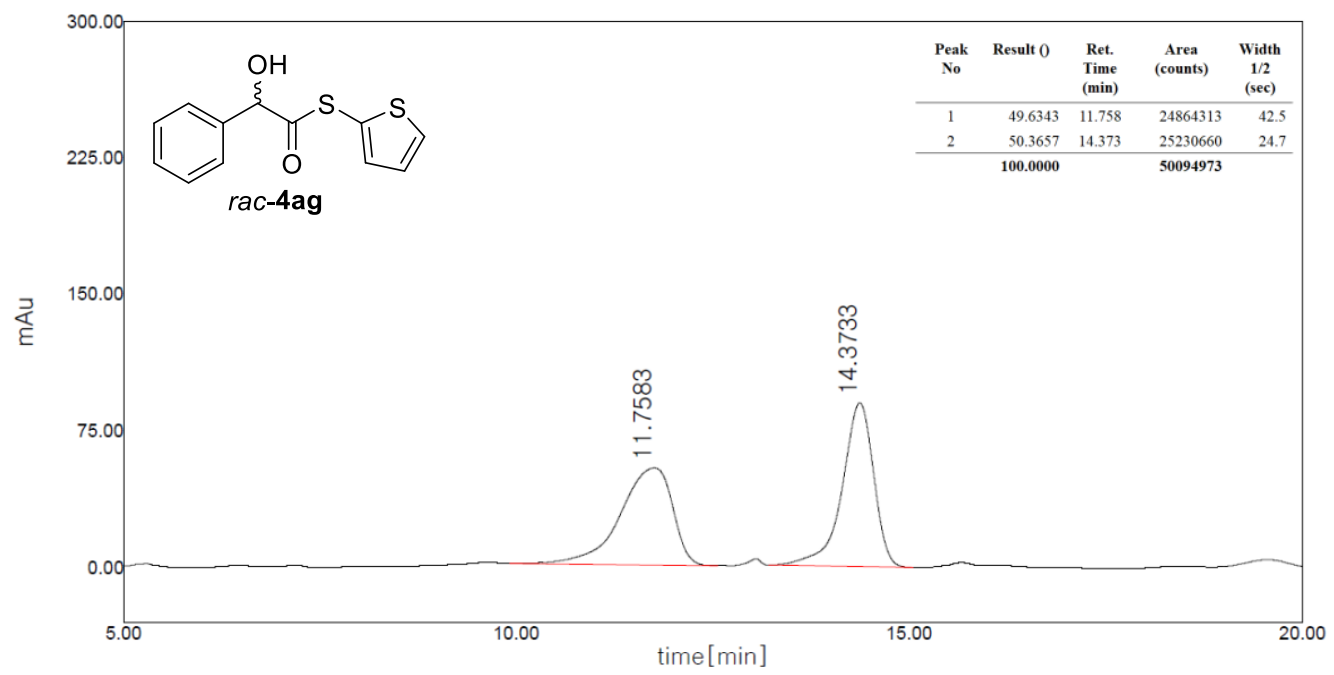

HPLC trace of $r a c-4 a g$

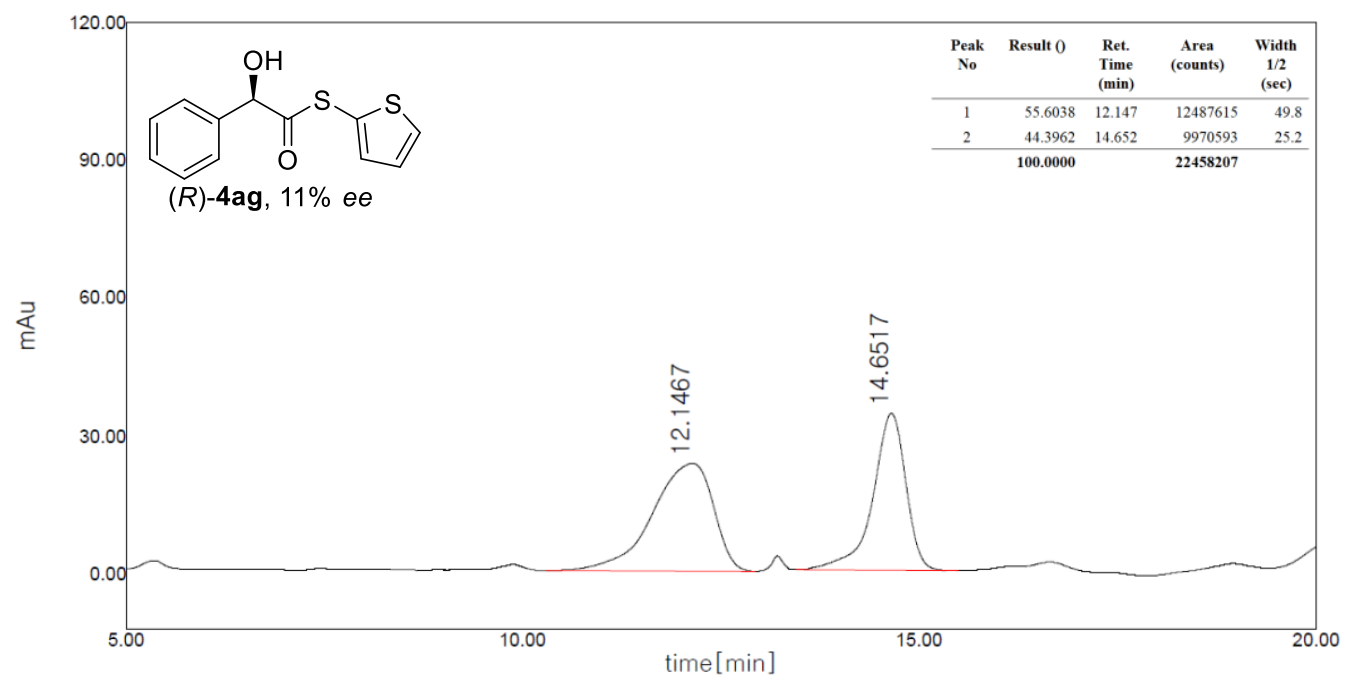

HPLC trace of $\mathbf{4 a g}-10$ equiv. of $\mathbf{2 a} / 10$ equiv. of eucalyptol 
HPLC traces of Scheme 2C

10 equiv. of thiol/10 equiv. of eucalyptol

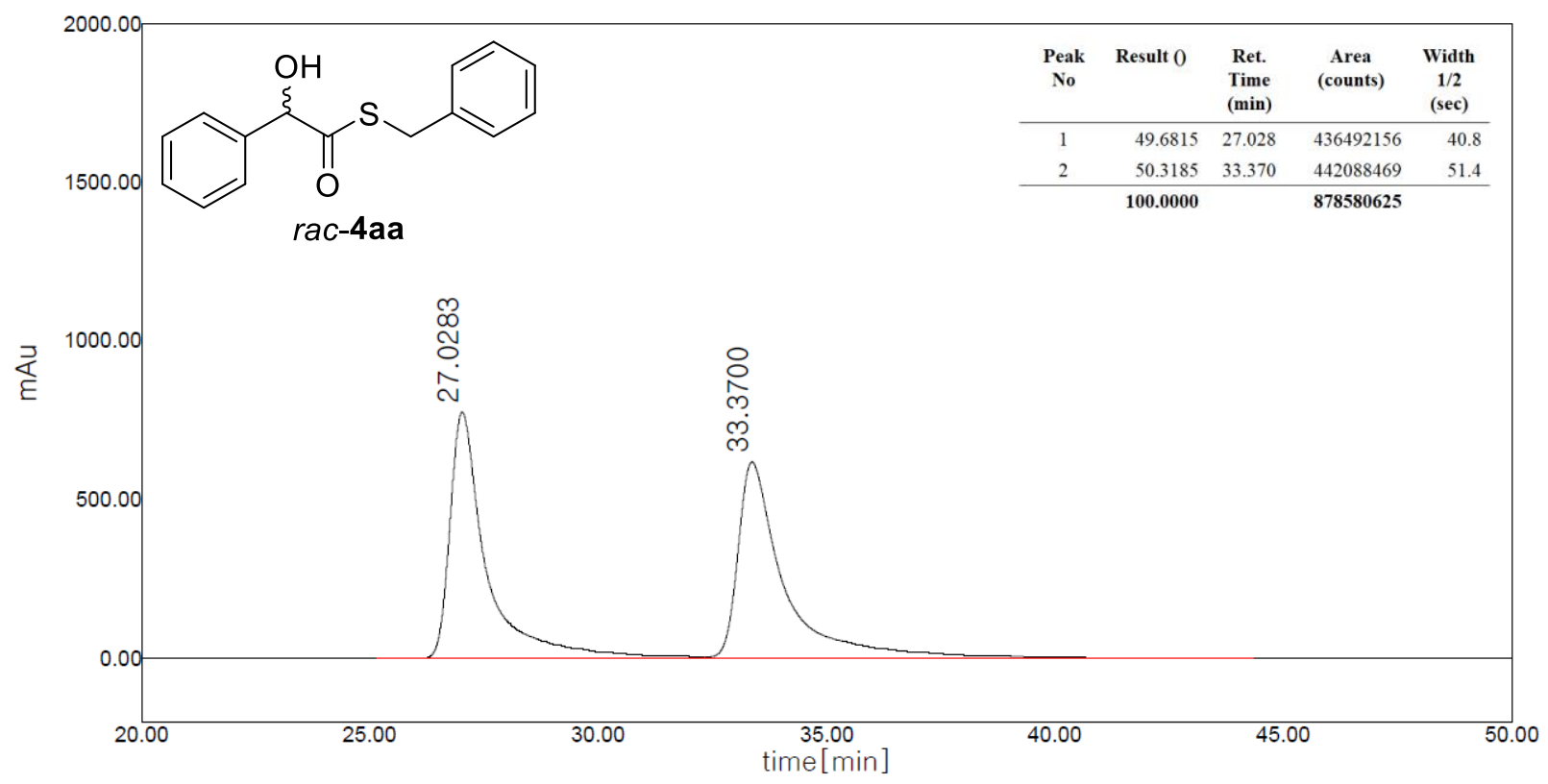

HPLC trace of $r a c-\mathbf{4 a a}$

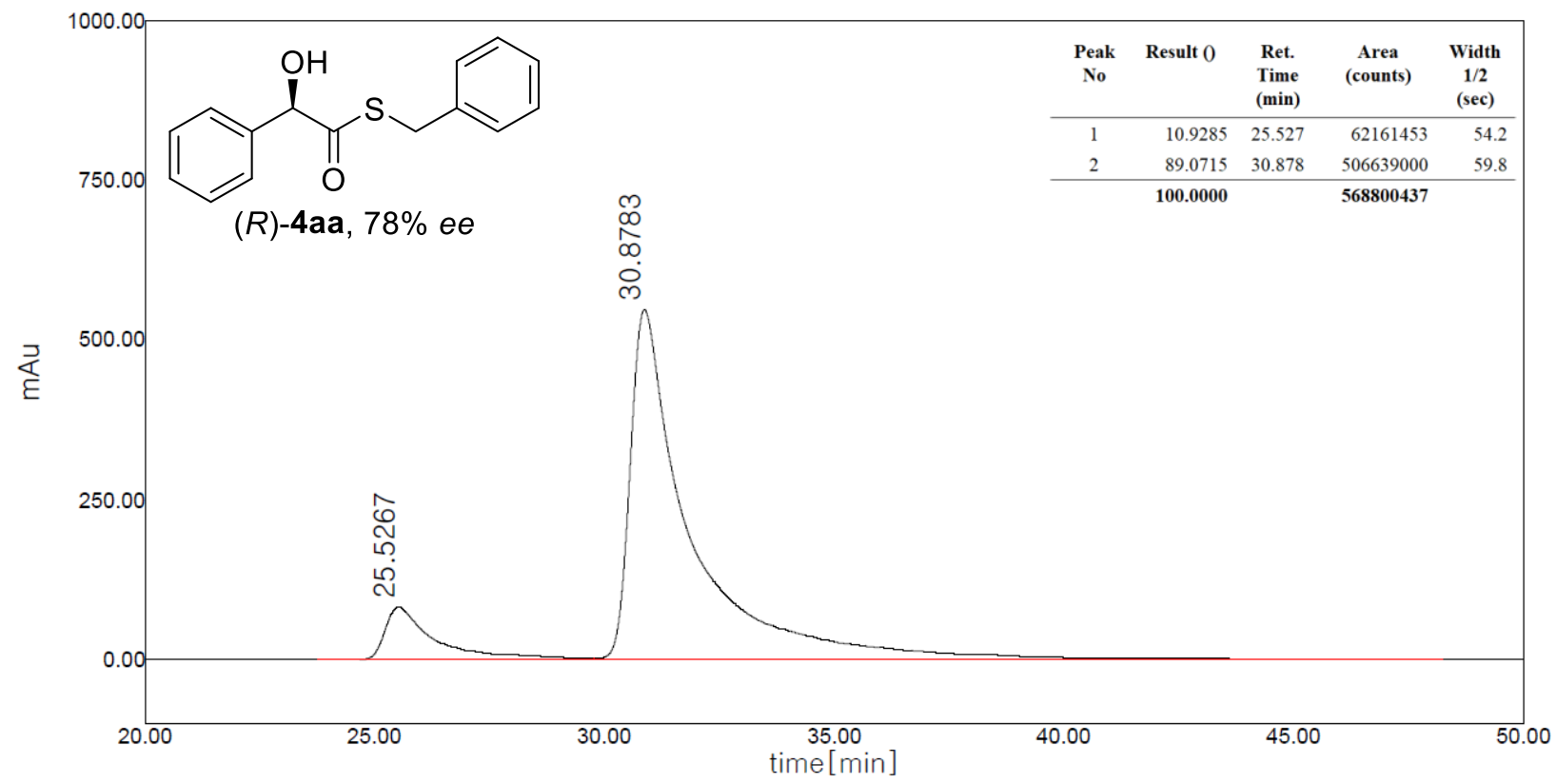

HPLC trace of 4aa (condition a) 


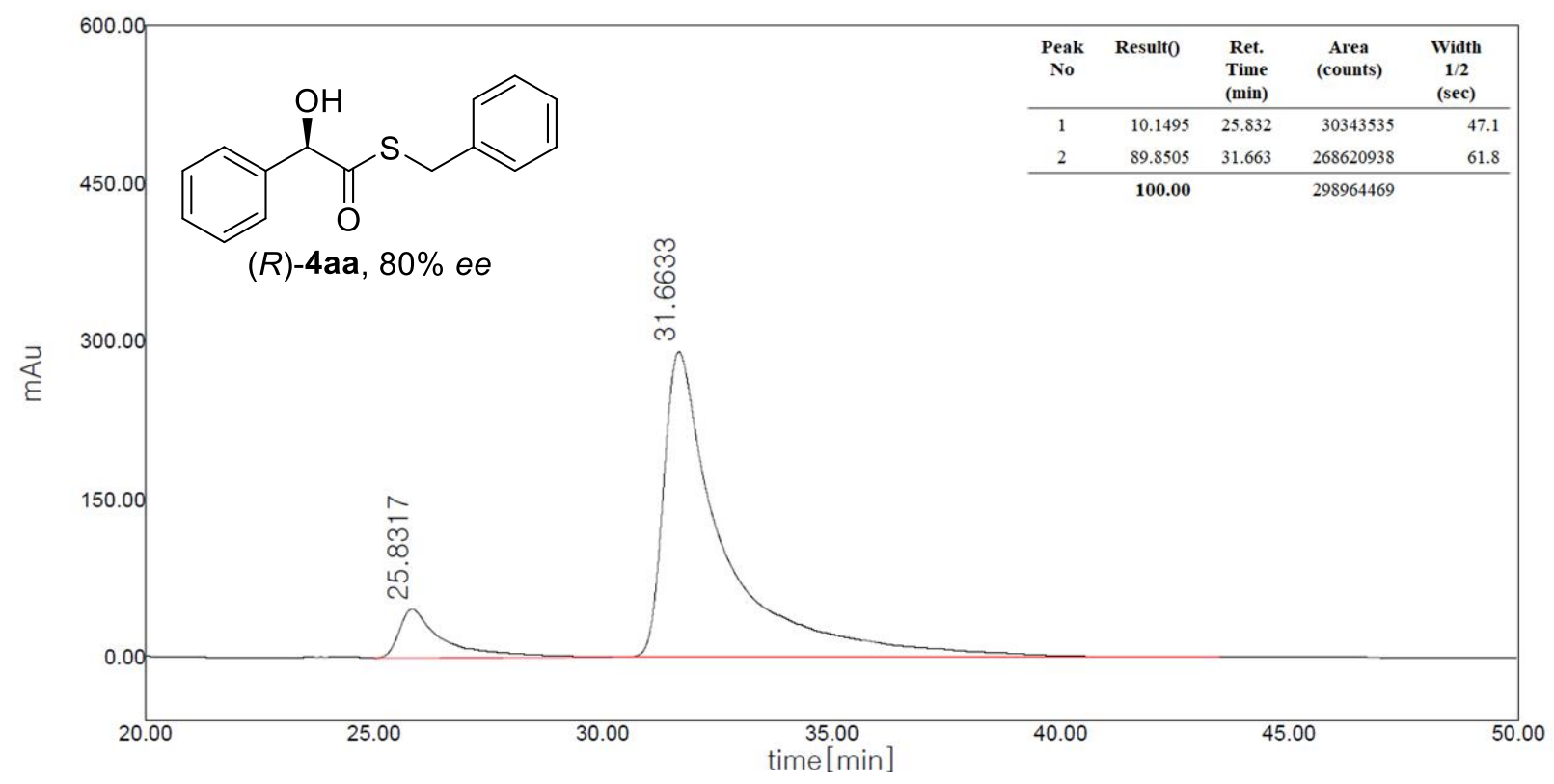

HPLC trace of 4aa (condition b)

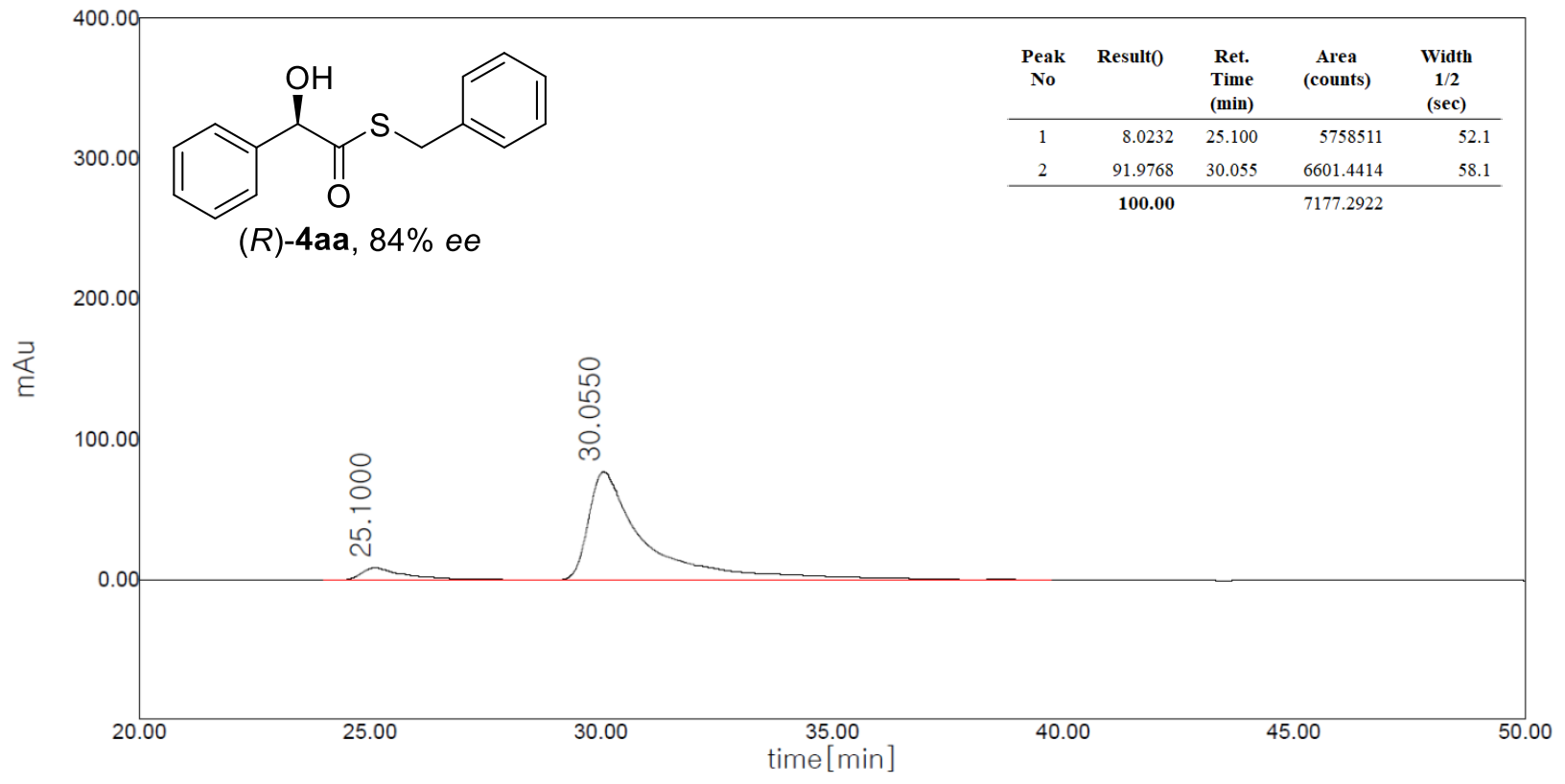

HPLC trace of 4aa (condition c) 


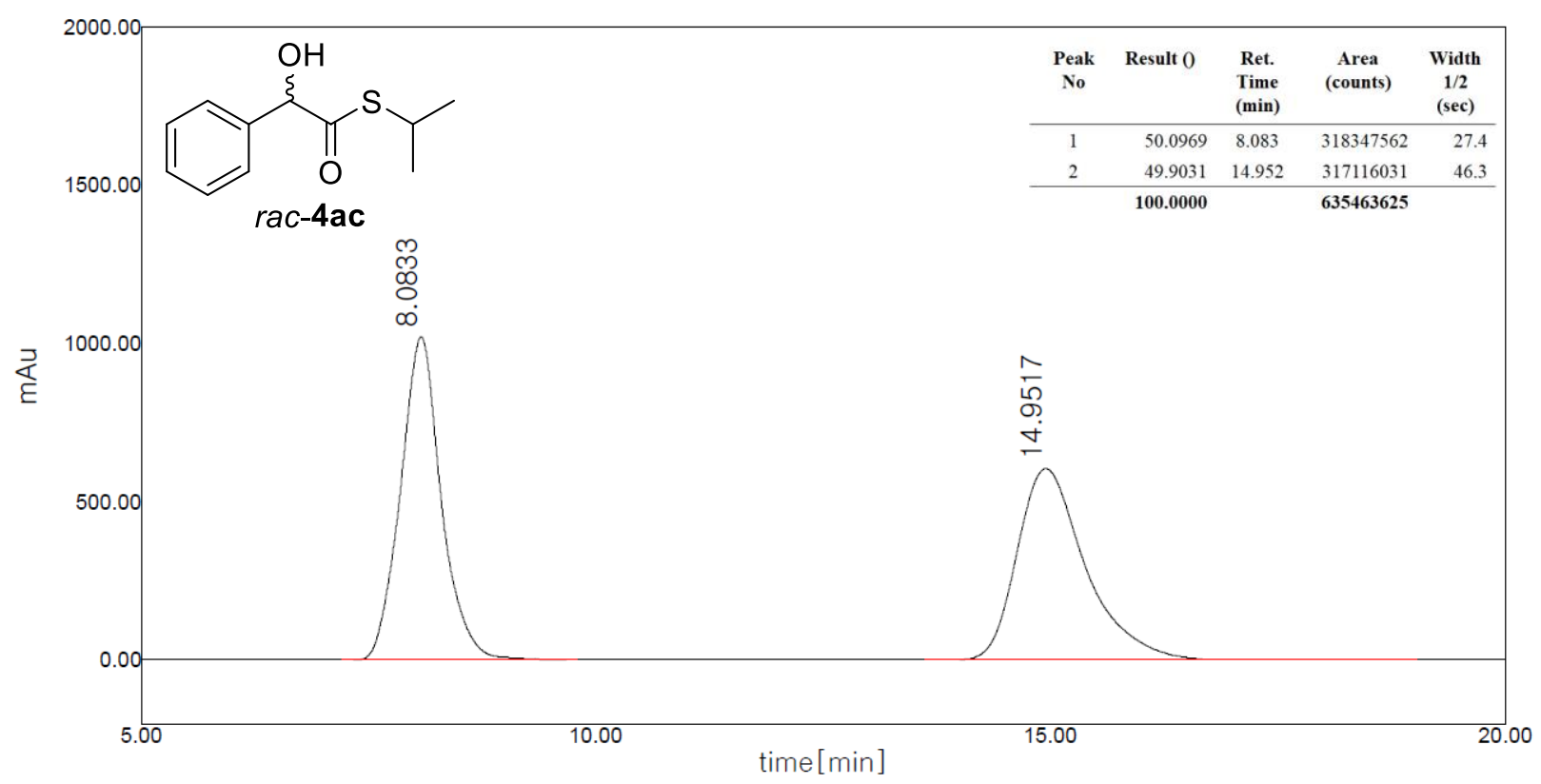

\section{HPLC trace of rac-4ac}

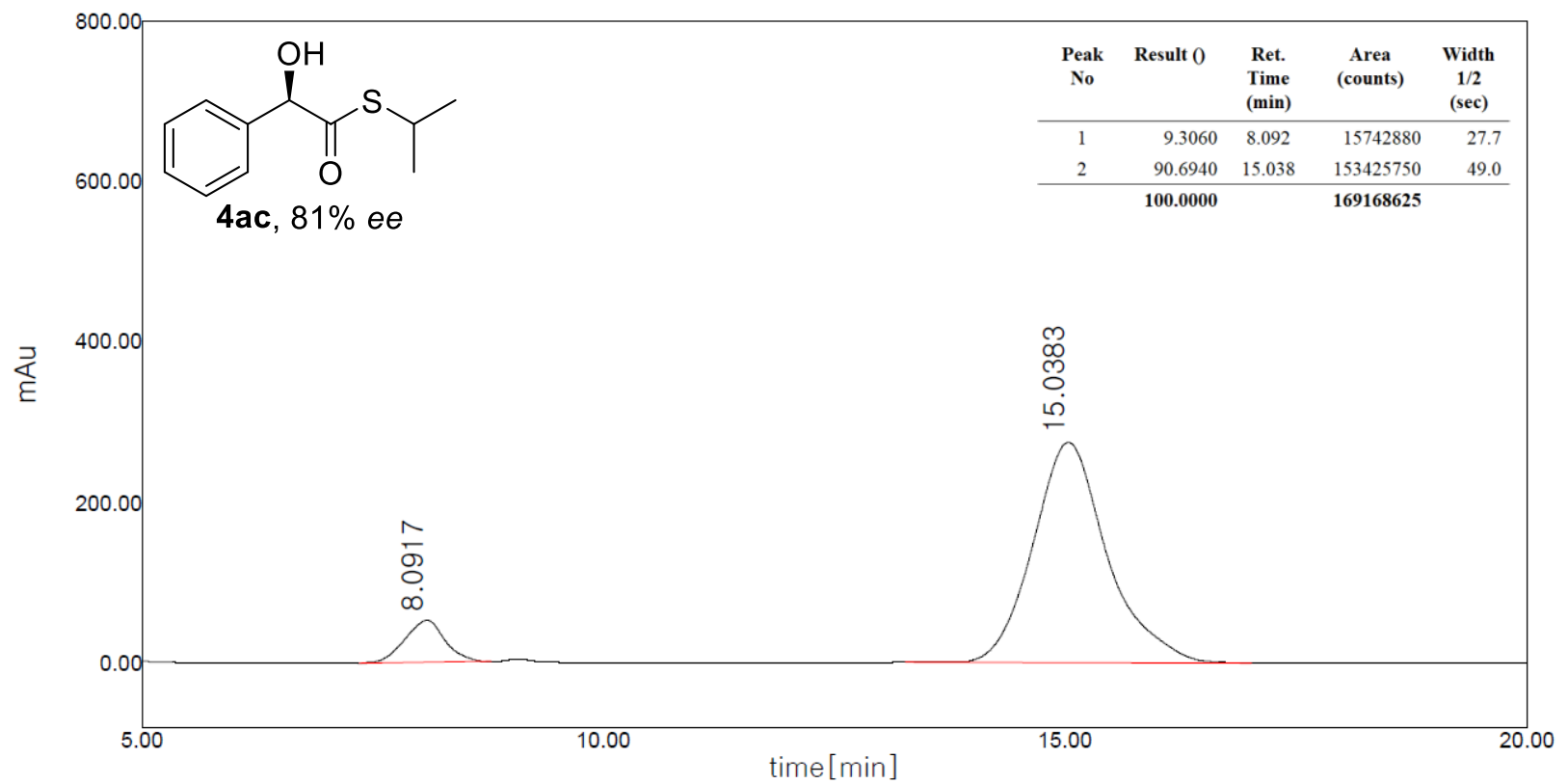

HPLC trace of 4ac (condition a) 


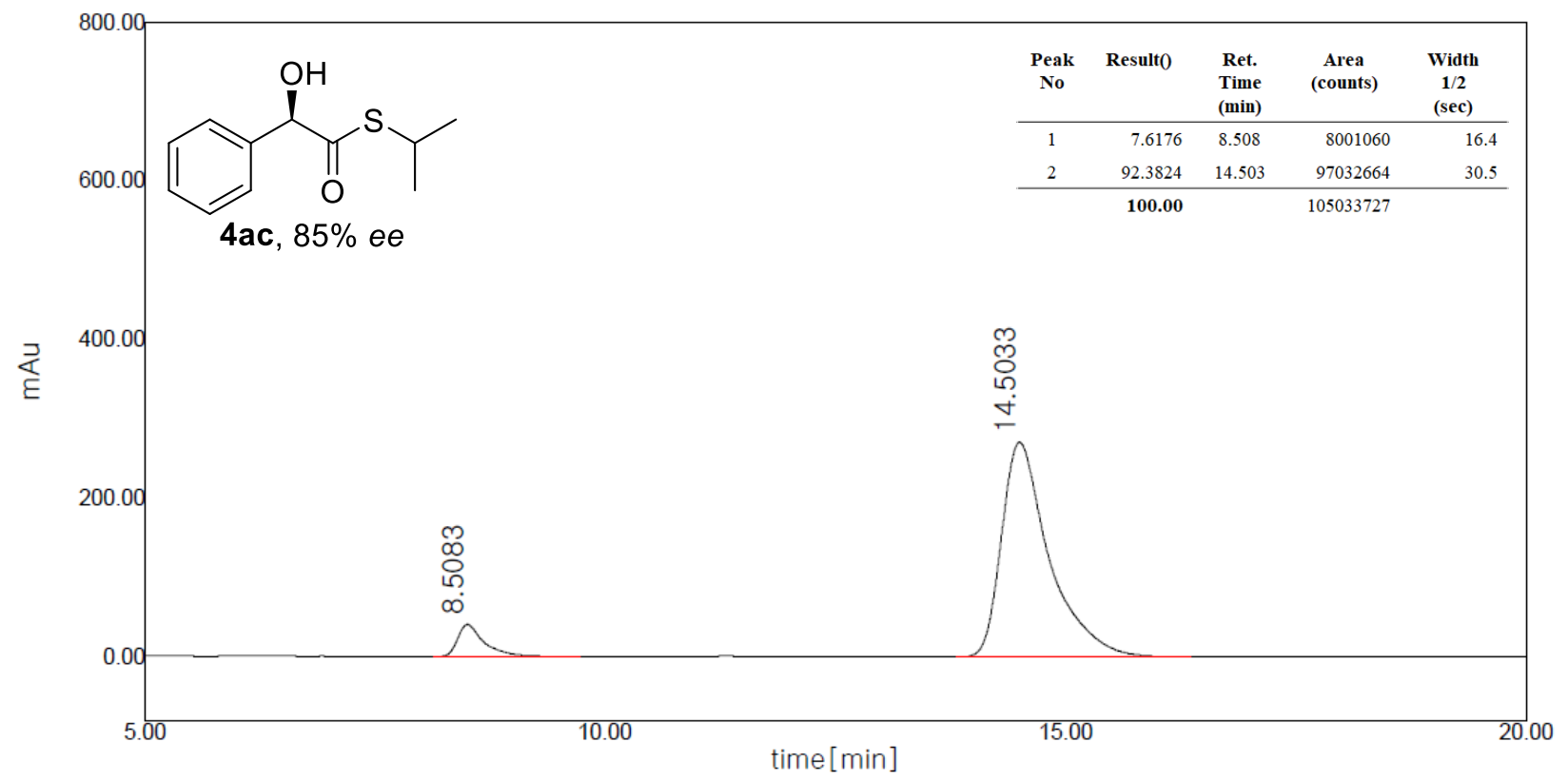

\section{HPLC trace of 4ac (condition b)}

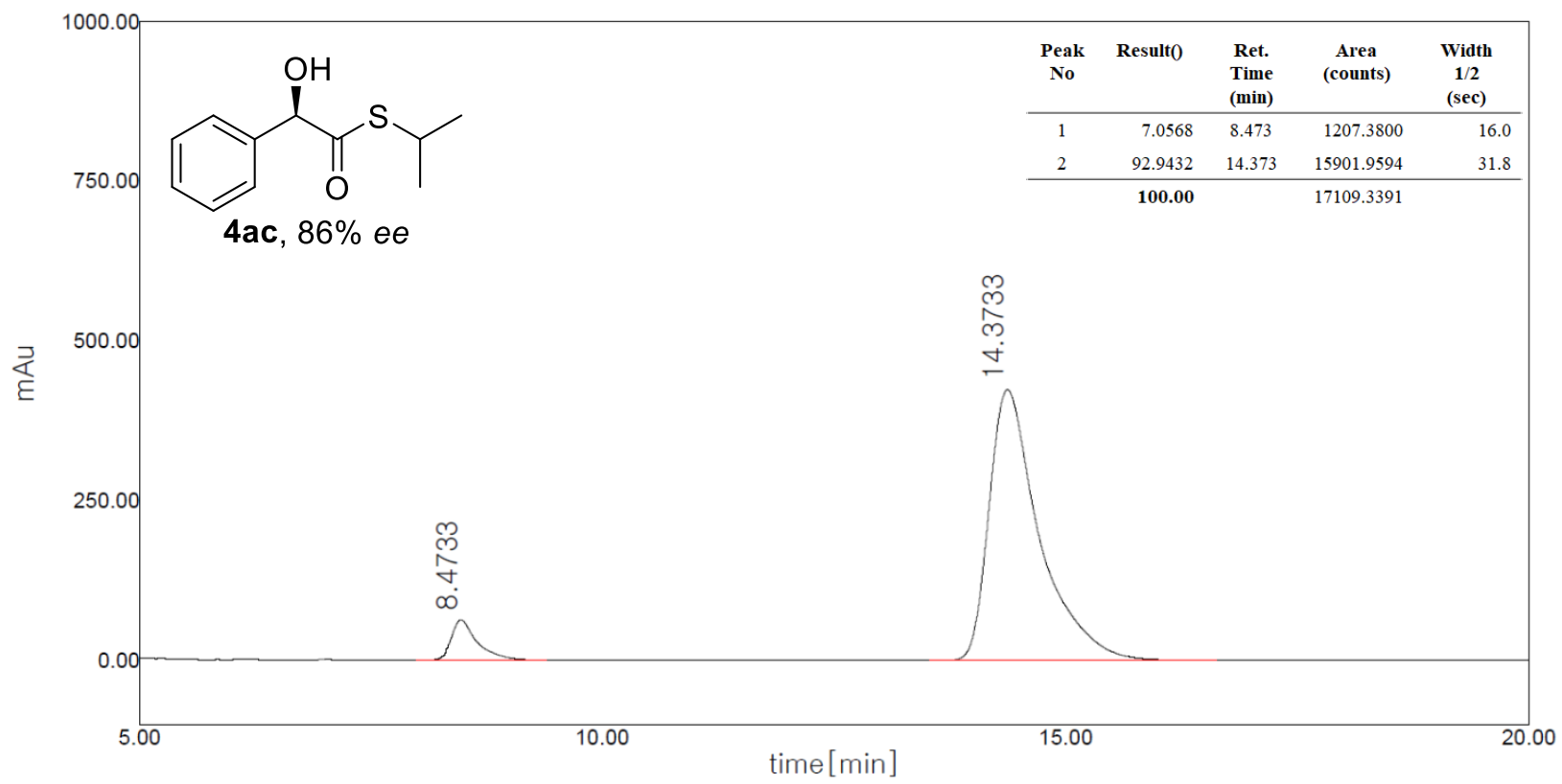

HPLC trace of 4ac (condition c) 


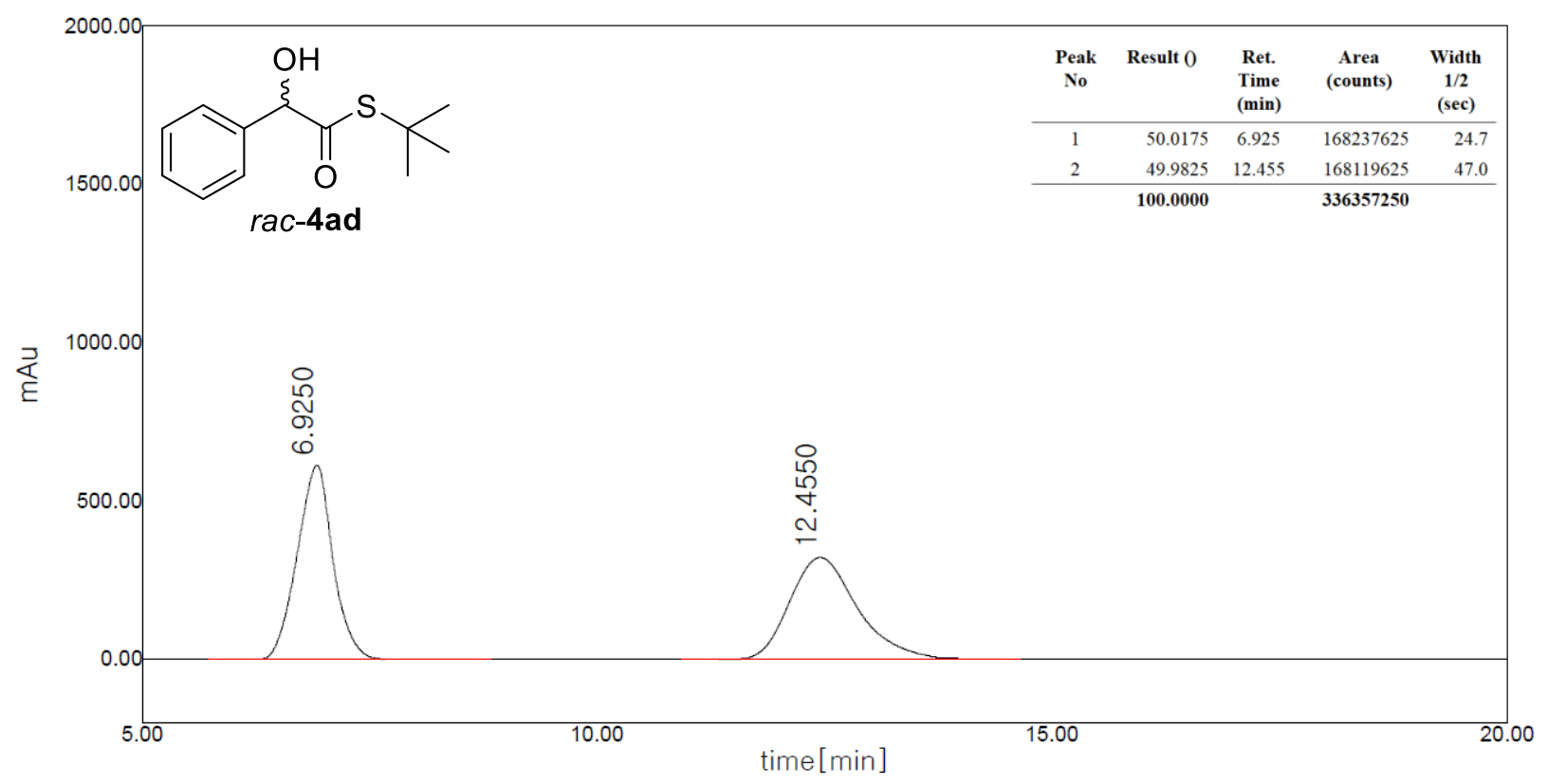

HPLC trace of $r a c-4 a d$

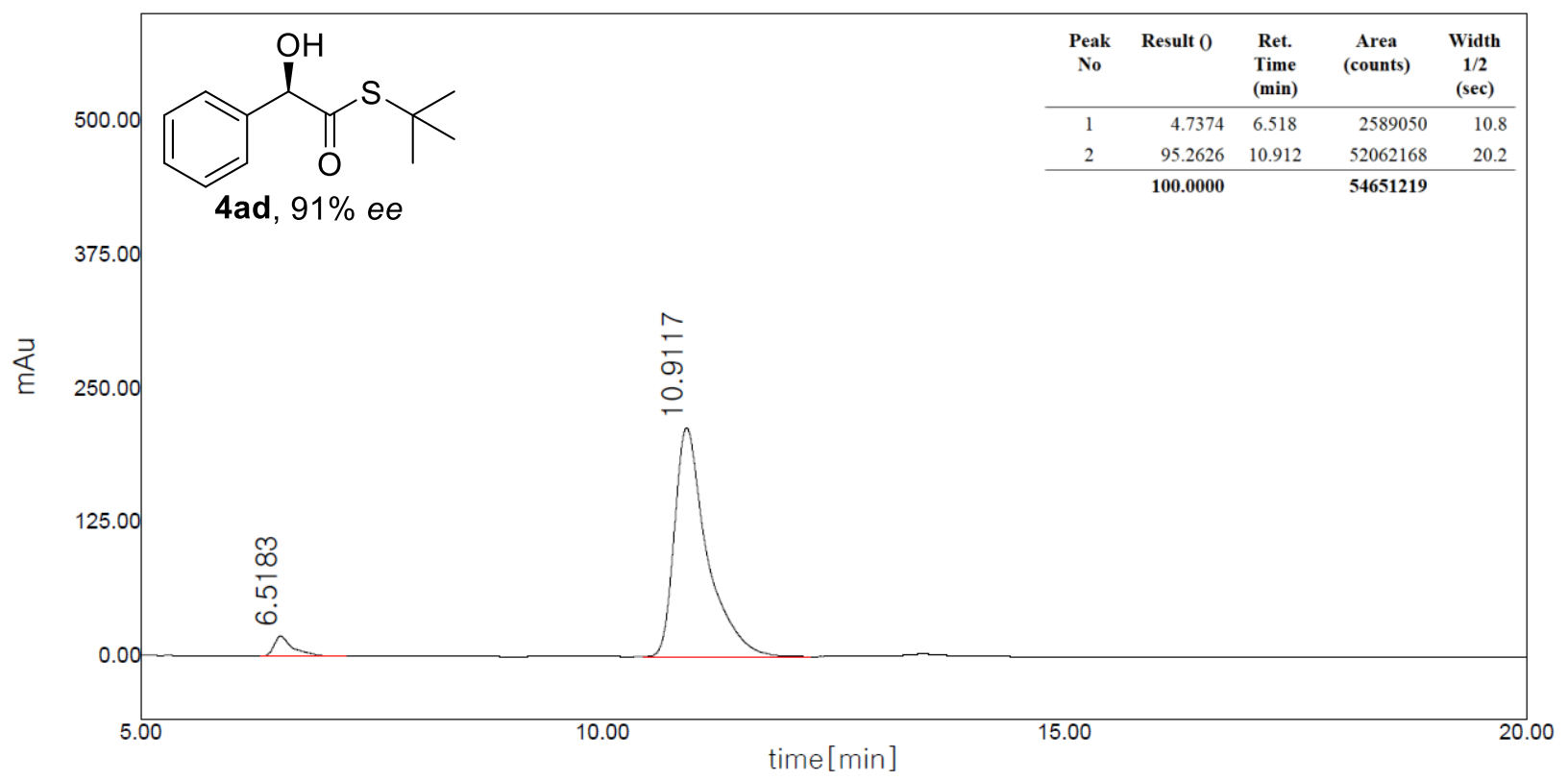

HPLC trace of 4ad (condition a) 


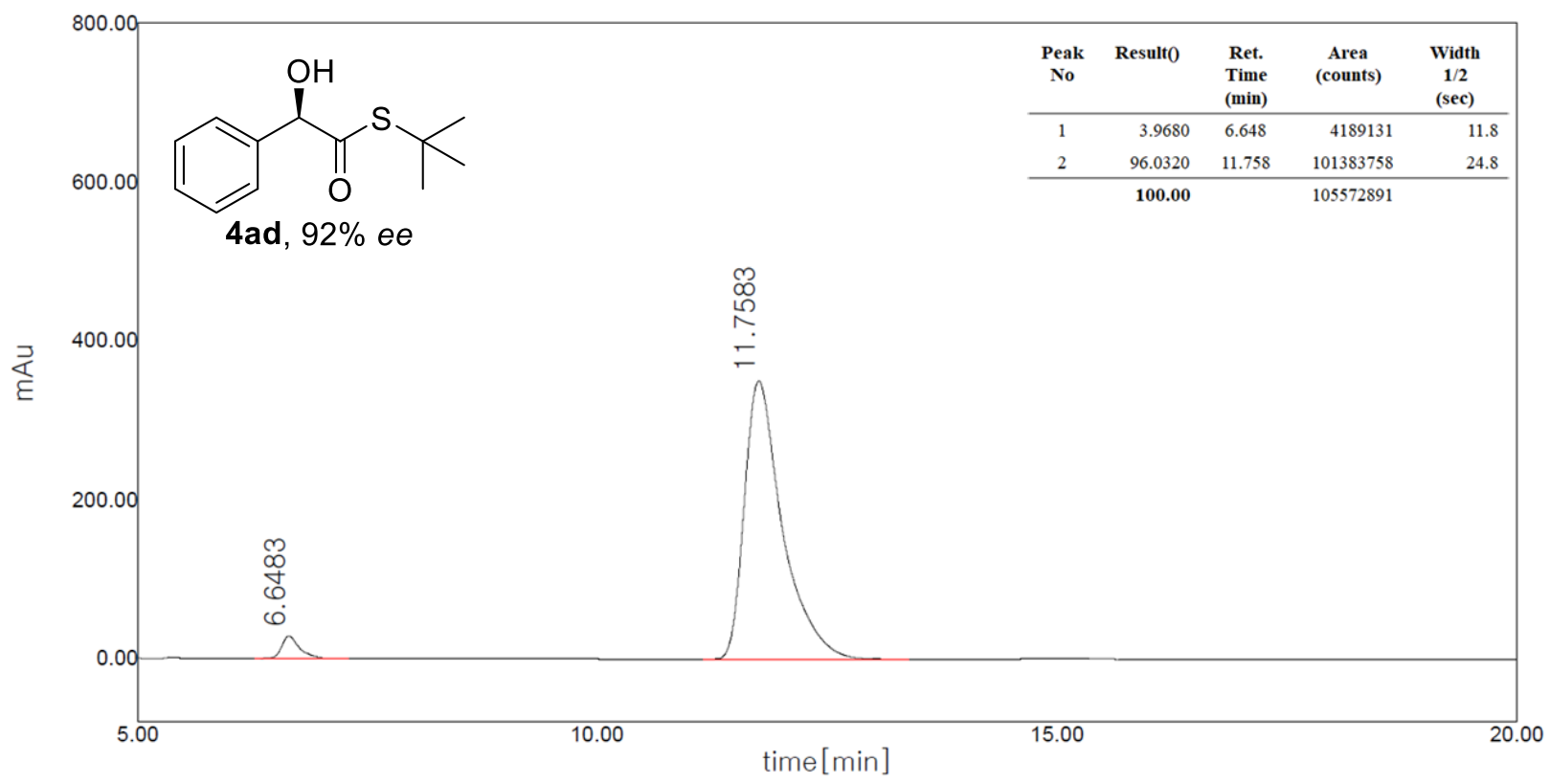

HPLC trace of $\mathbf{4 a d}$ (condition b)

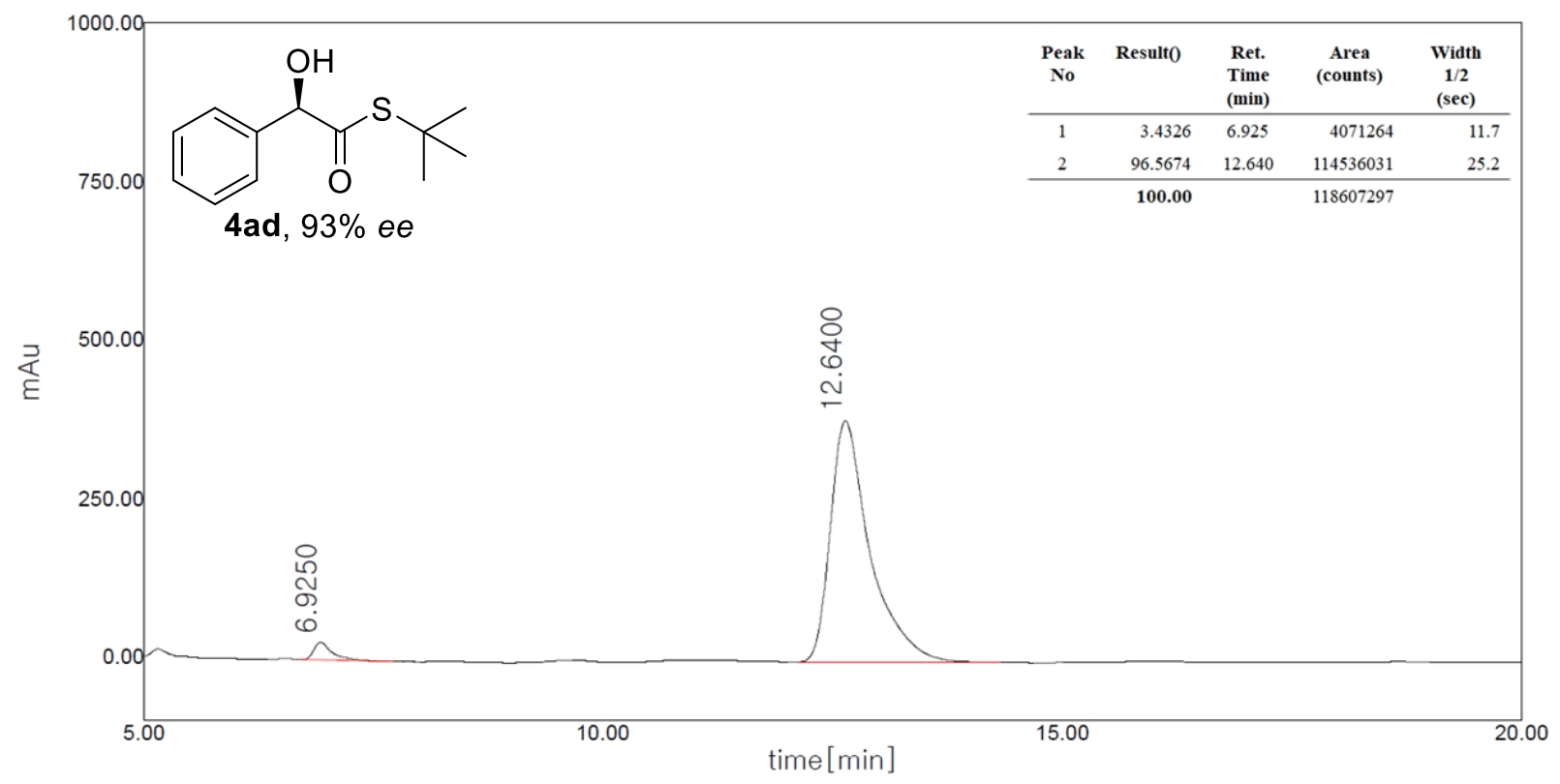

HPLC trace of $\mathbf{4 a d}$ (condition c) 
HPLC trace of Scheme 3

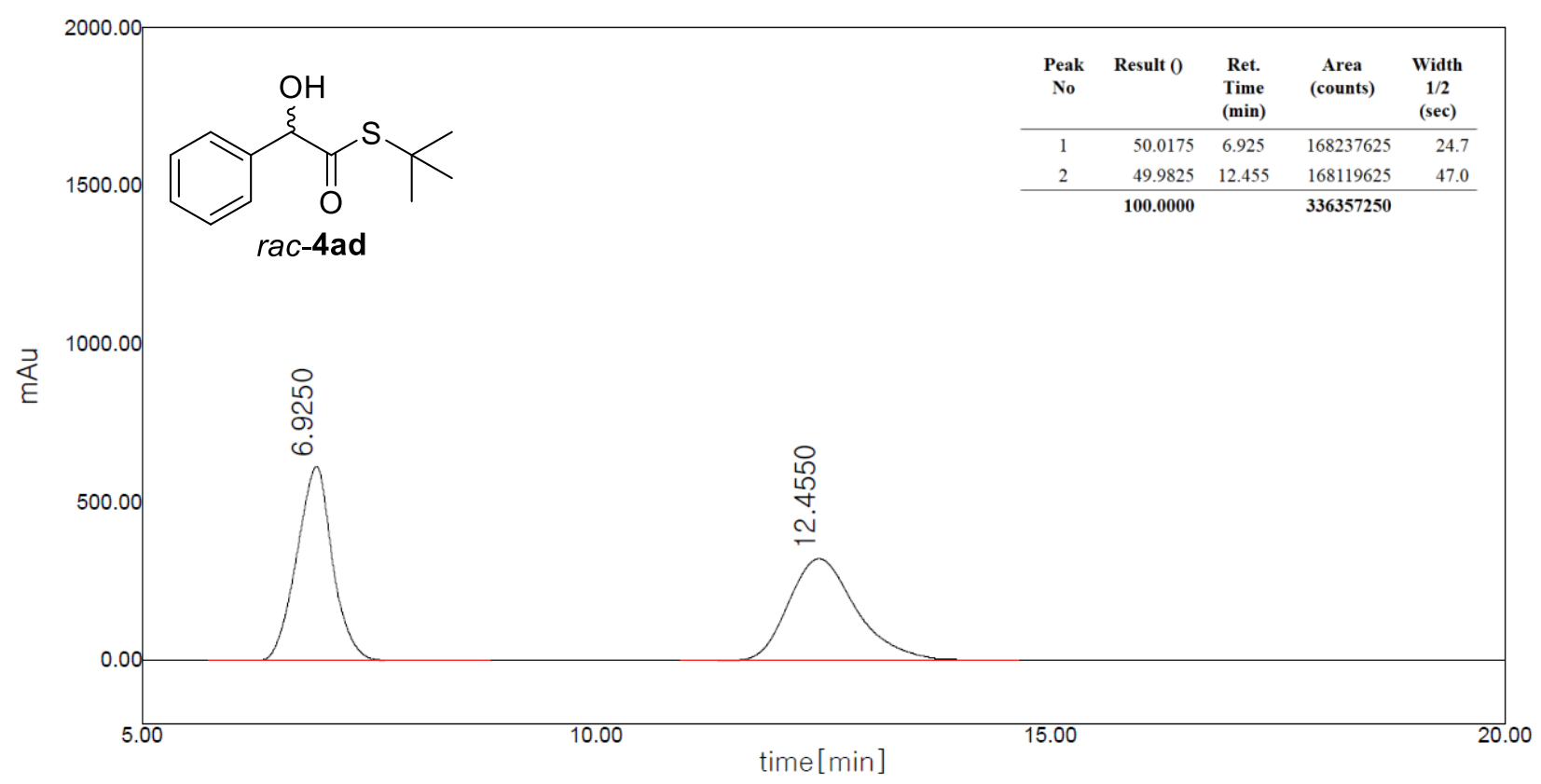

\section{HPLC trace of $r a c-4 a d$}

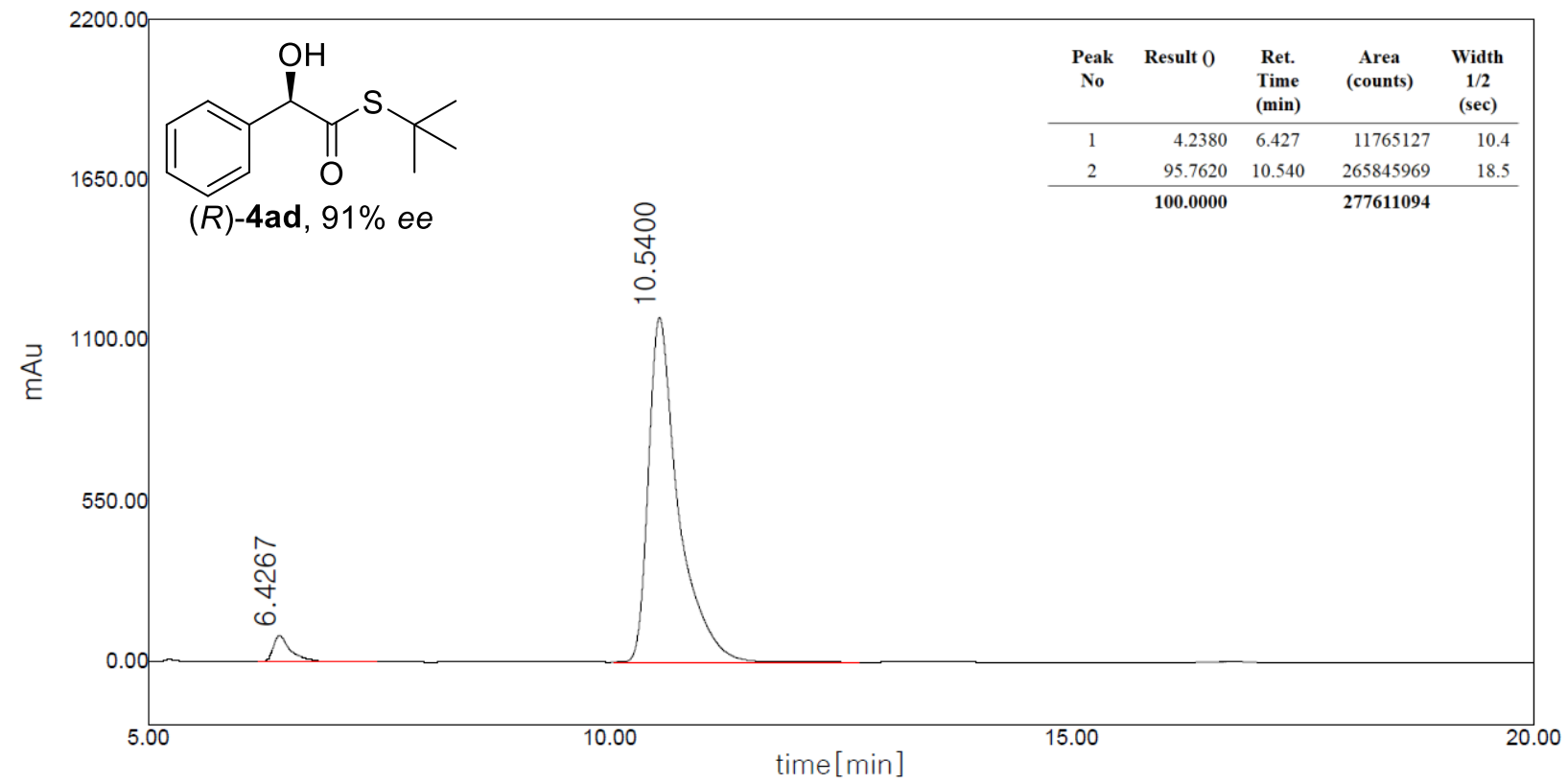

HPLC trace of 4ad (condition a) 


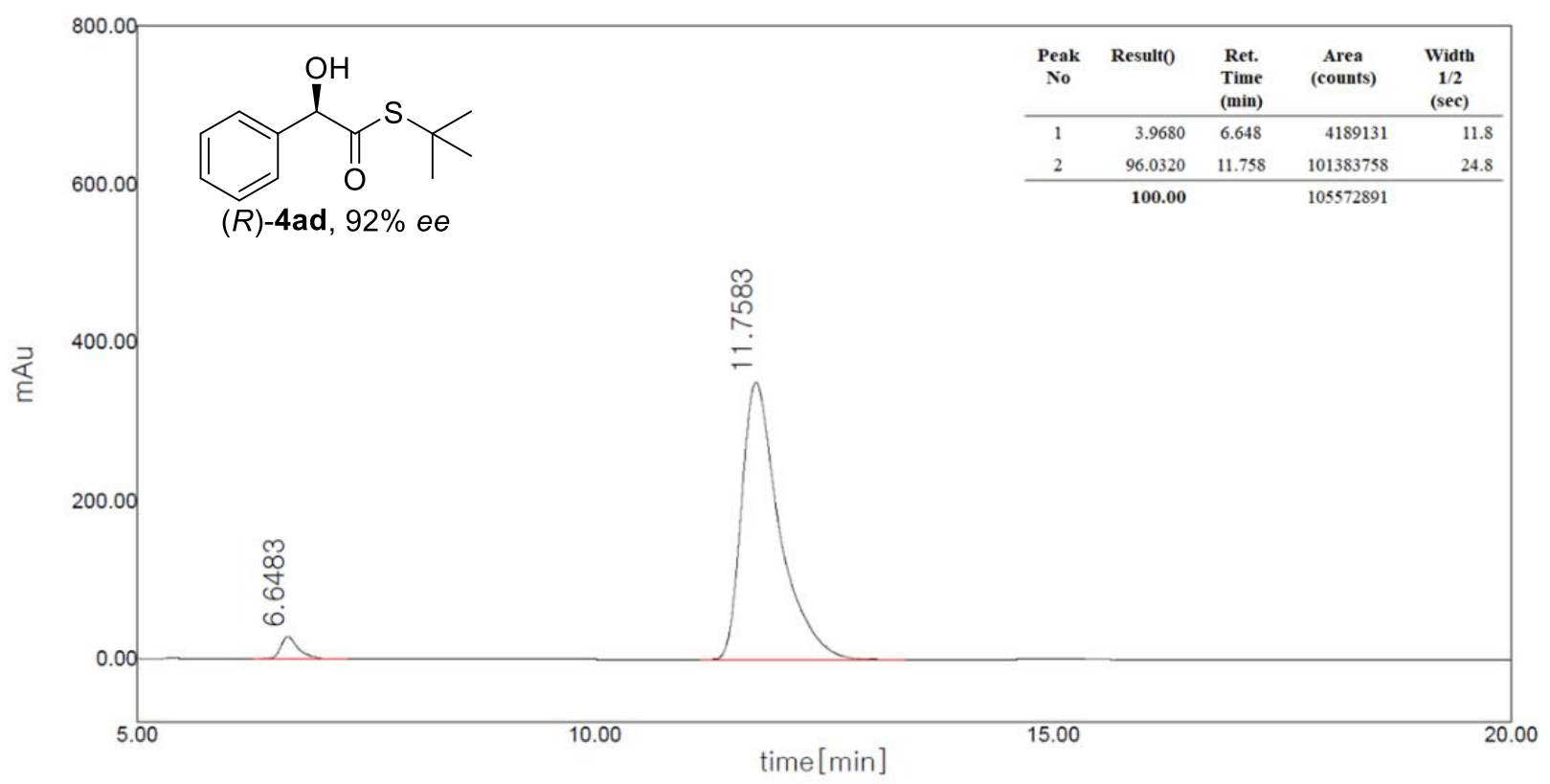

HPLC trace of 4ad (condition b)

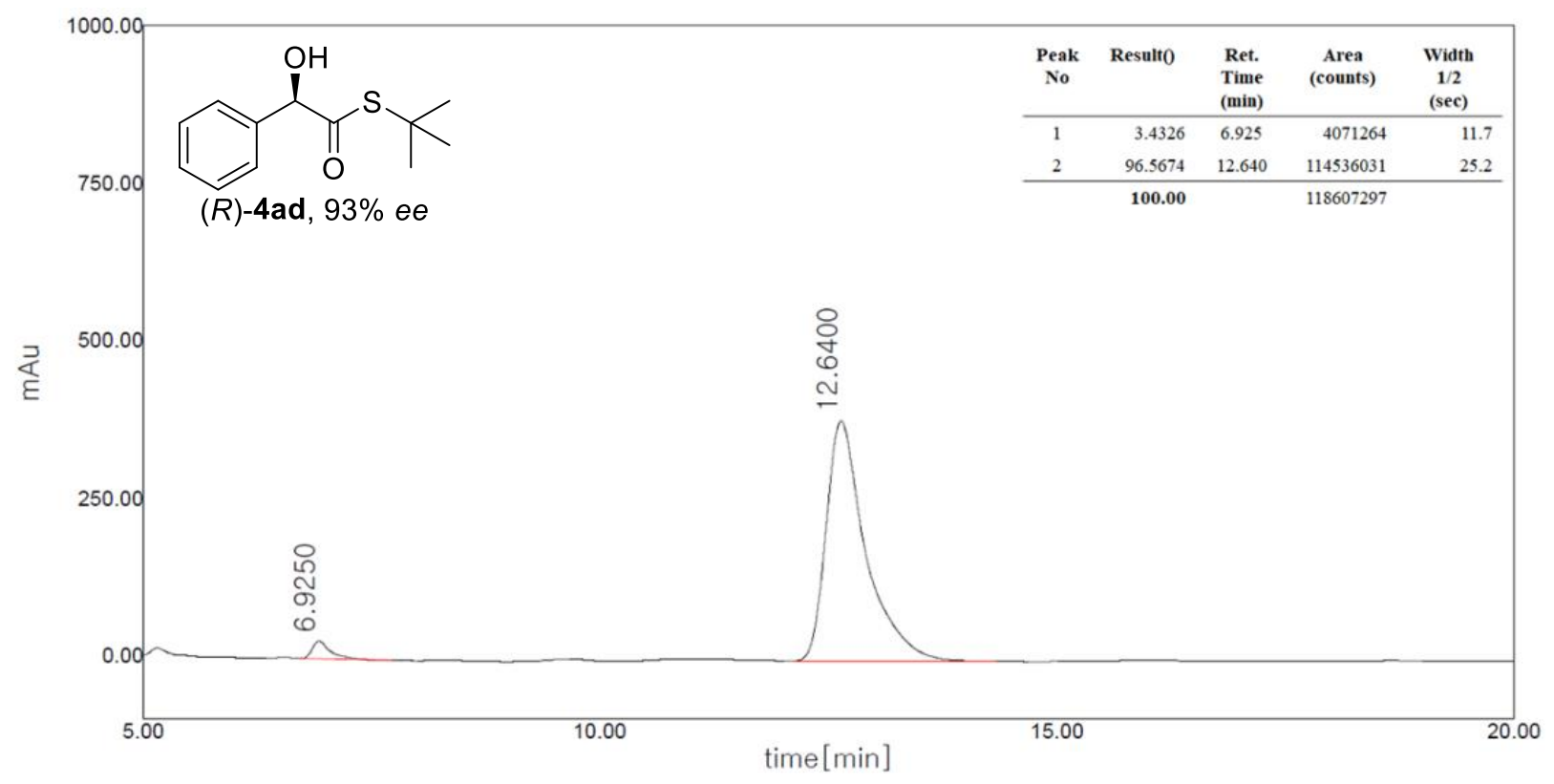

HPLC trace of 4ad (condition c) 


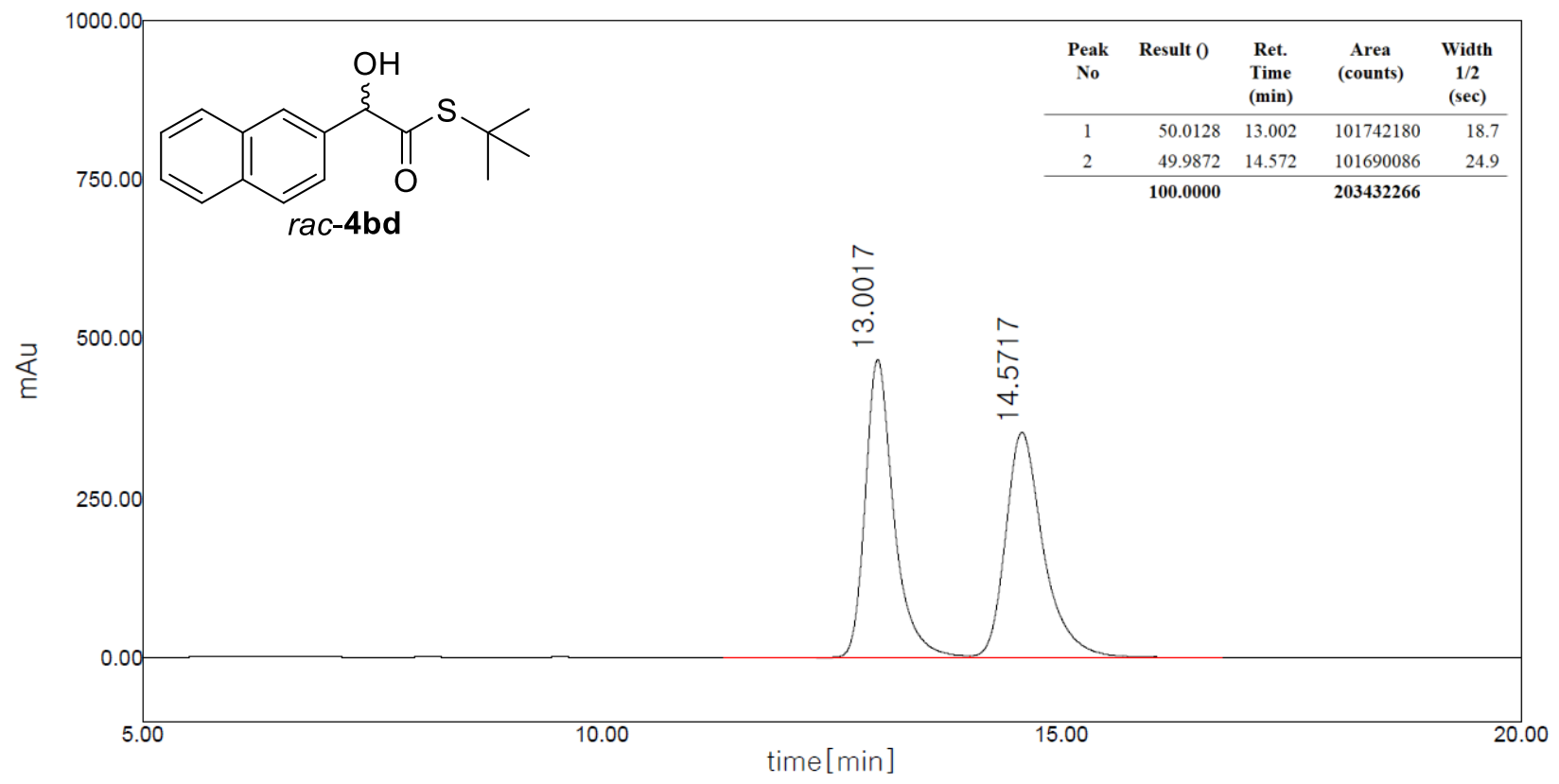

HPLC trace of $r a c-4 b d$

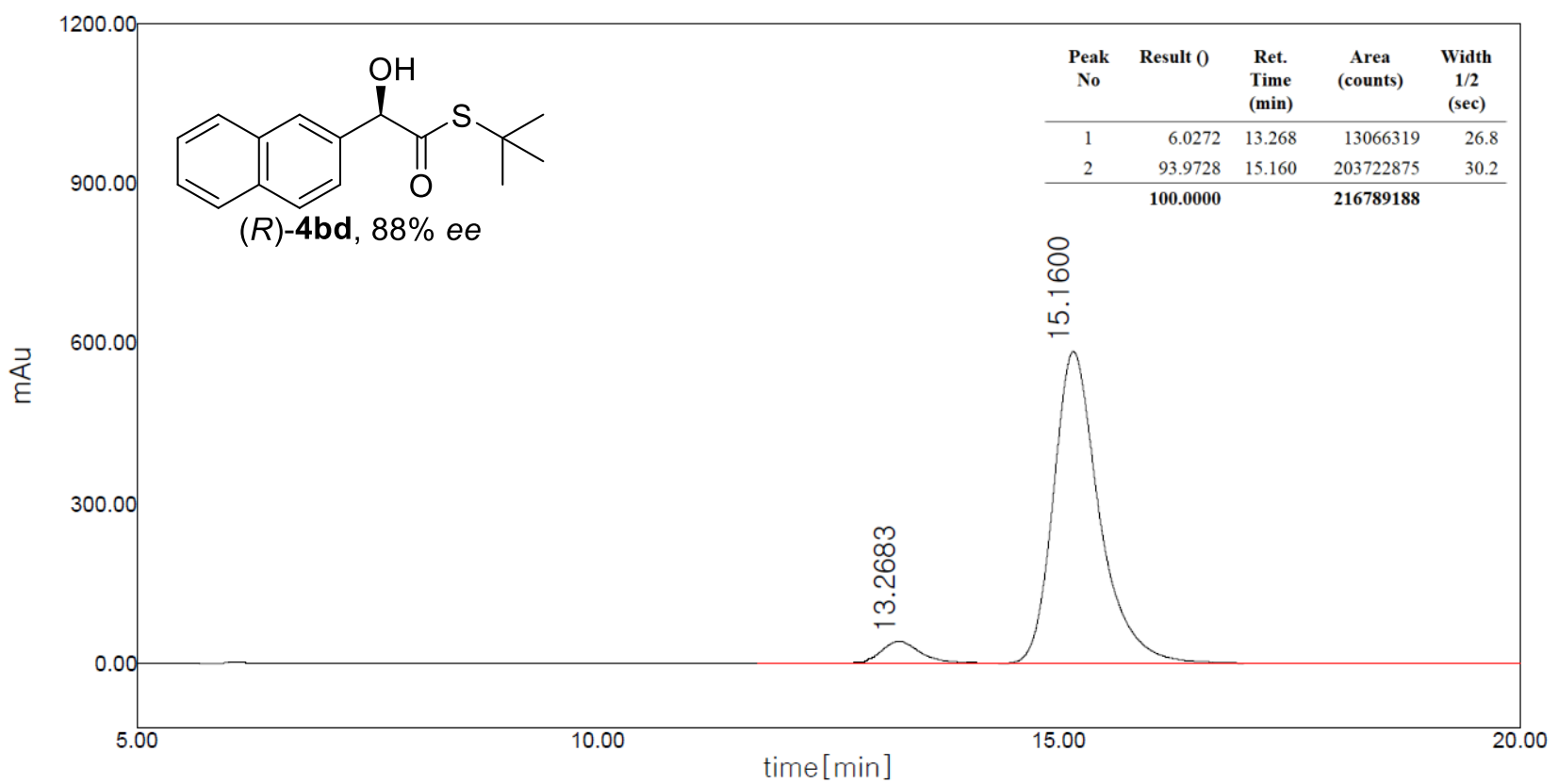

HPLC trace of 4bd (condition a) 


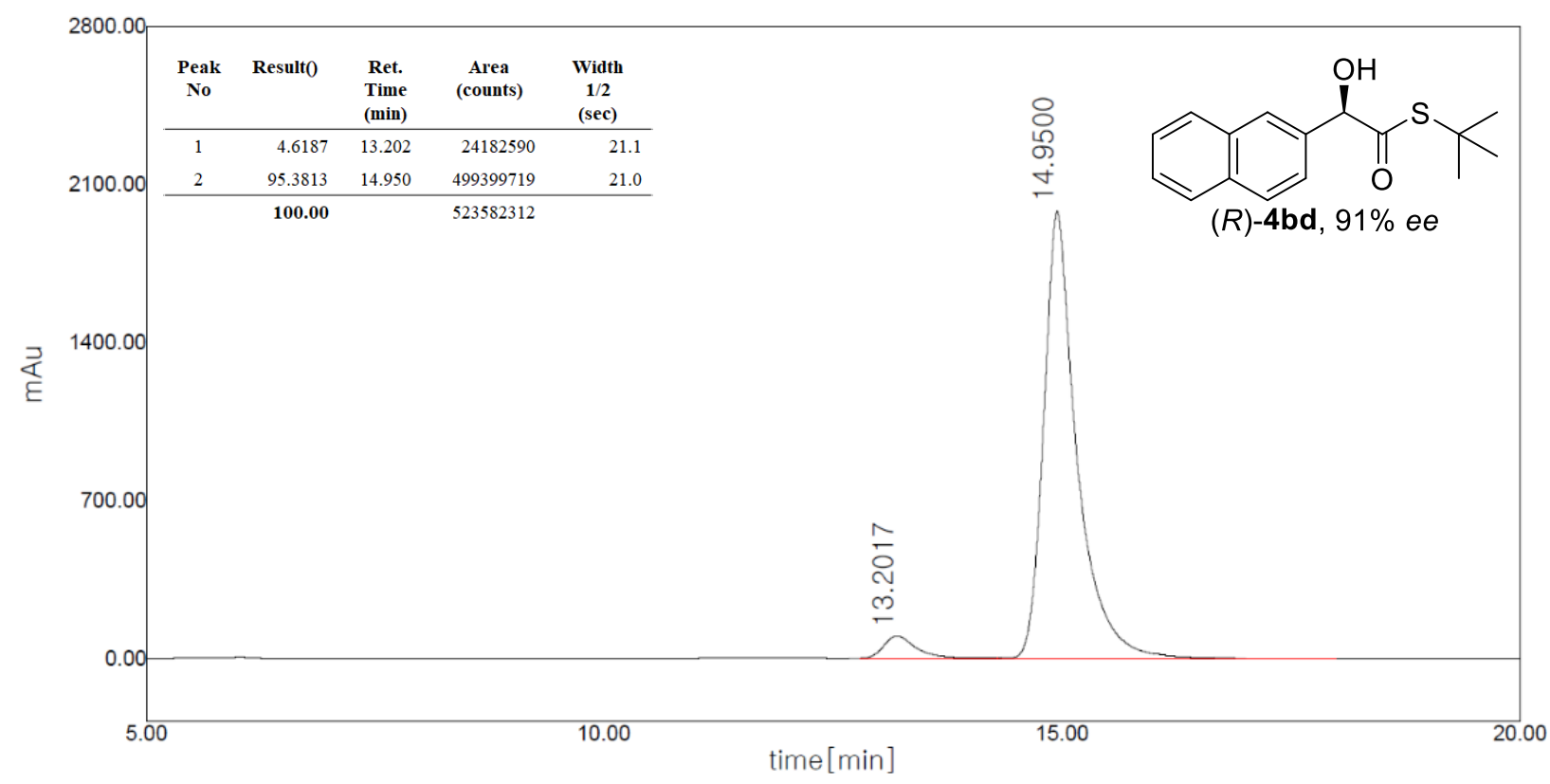

HPLC trace of $\mathbf{4 b d}$ (condition $b$ )

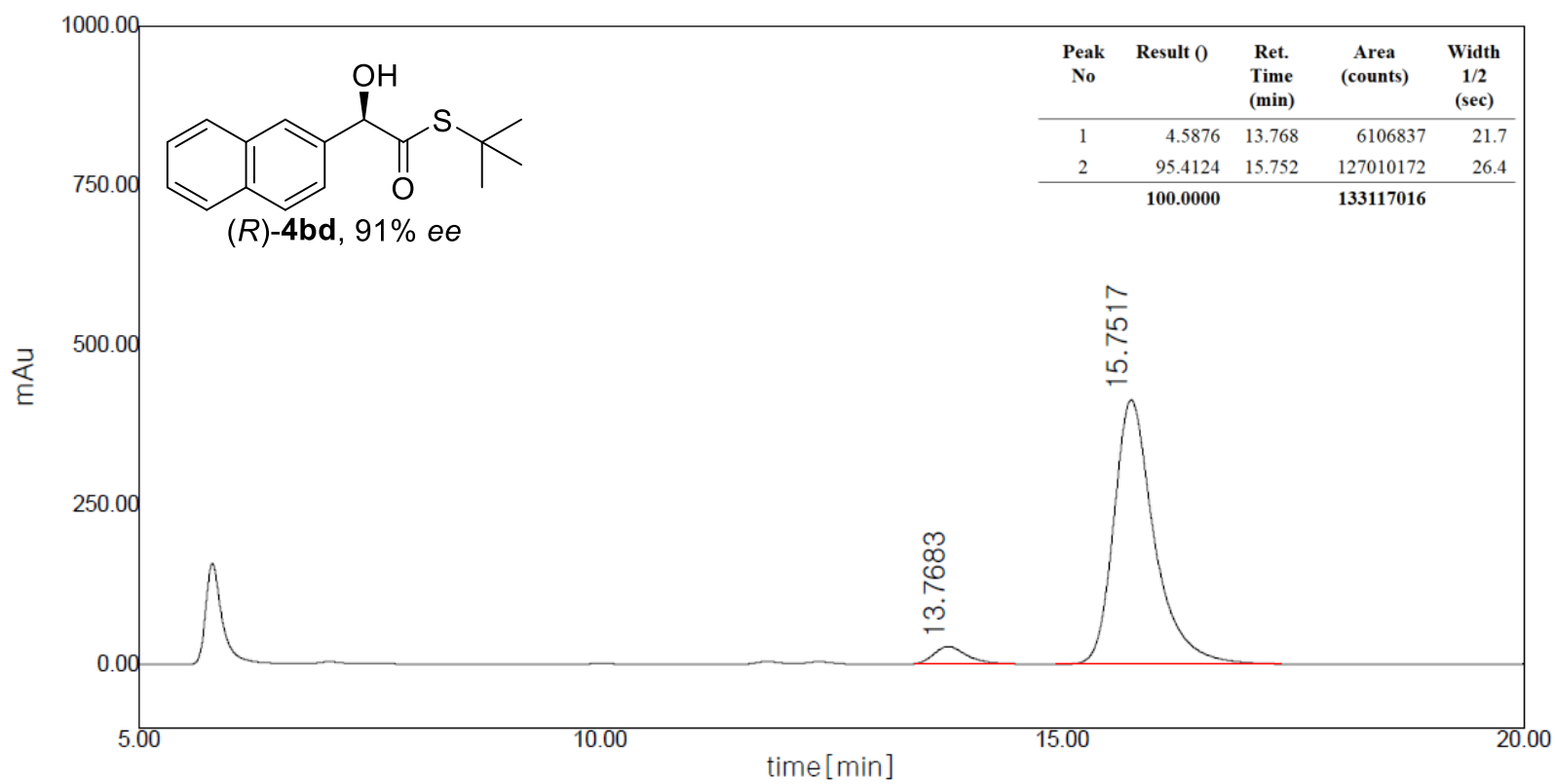

HPLC trace of $\mathbf{4 b d}$ (condition c) 


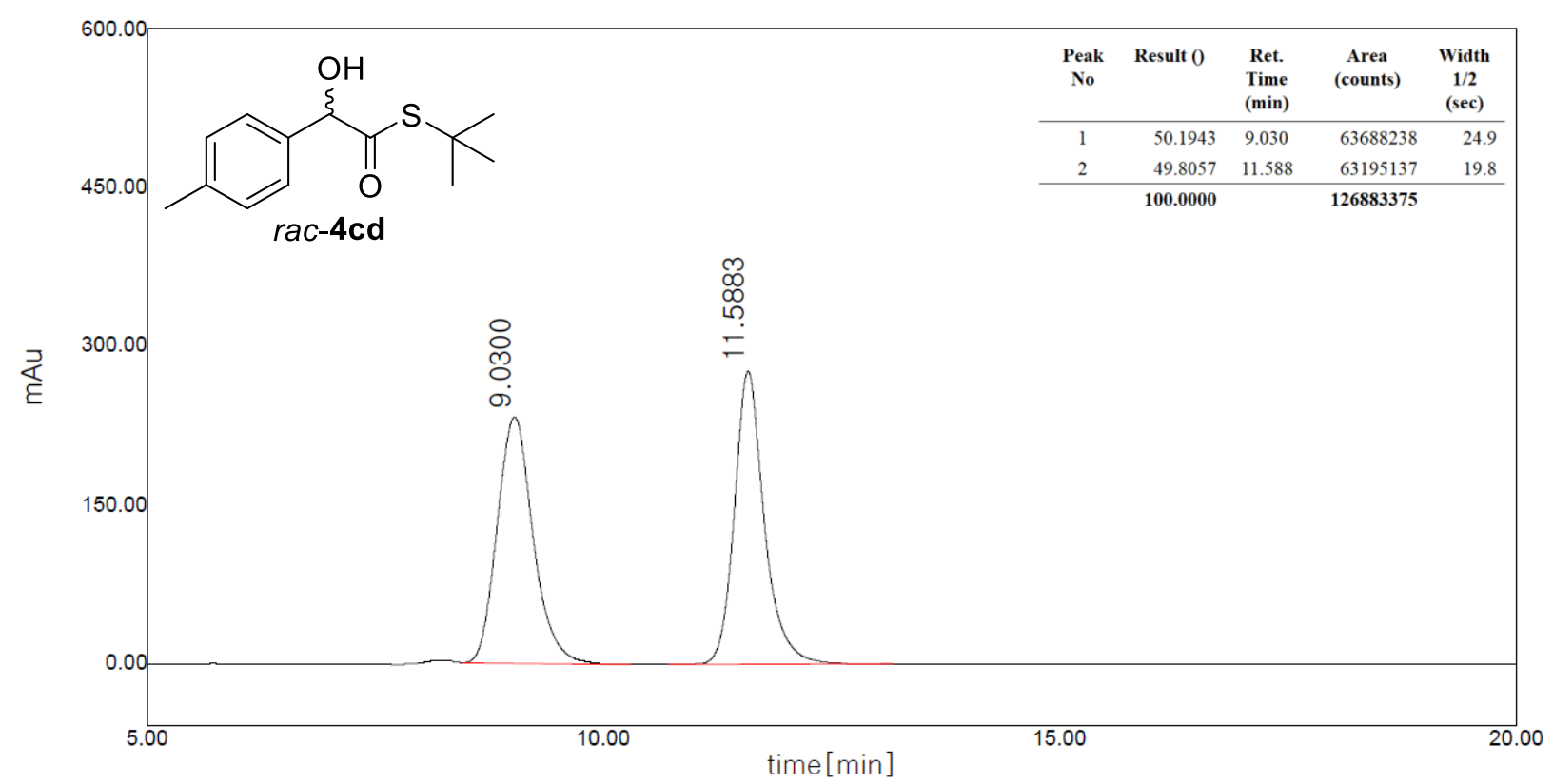

\section{HPLC trace of rac-4cd}

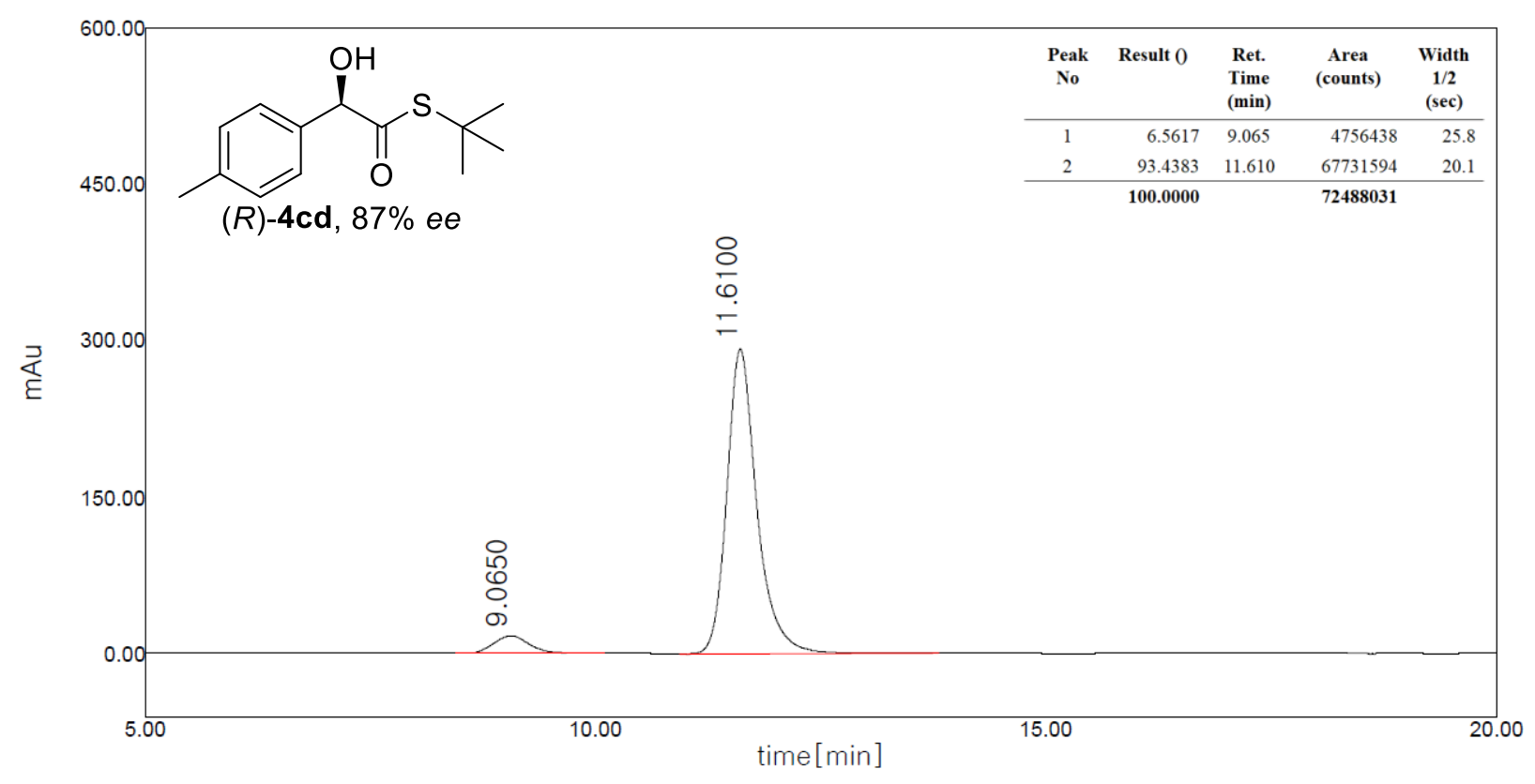

HPLC trace of 4cd (condition a) 


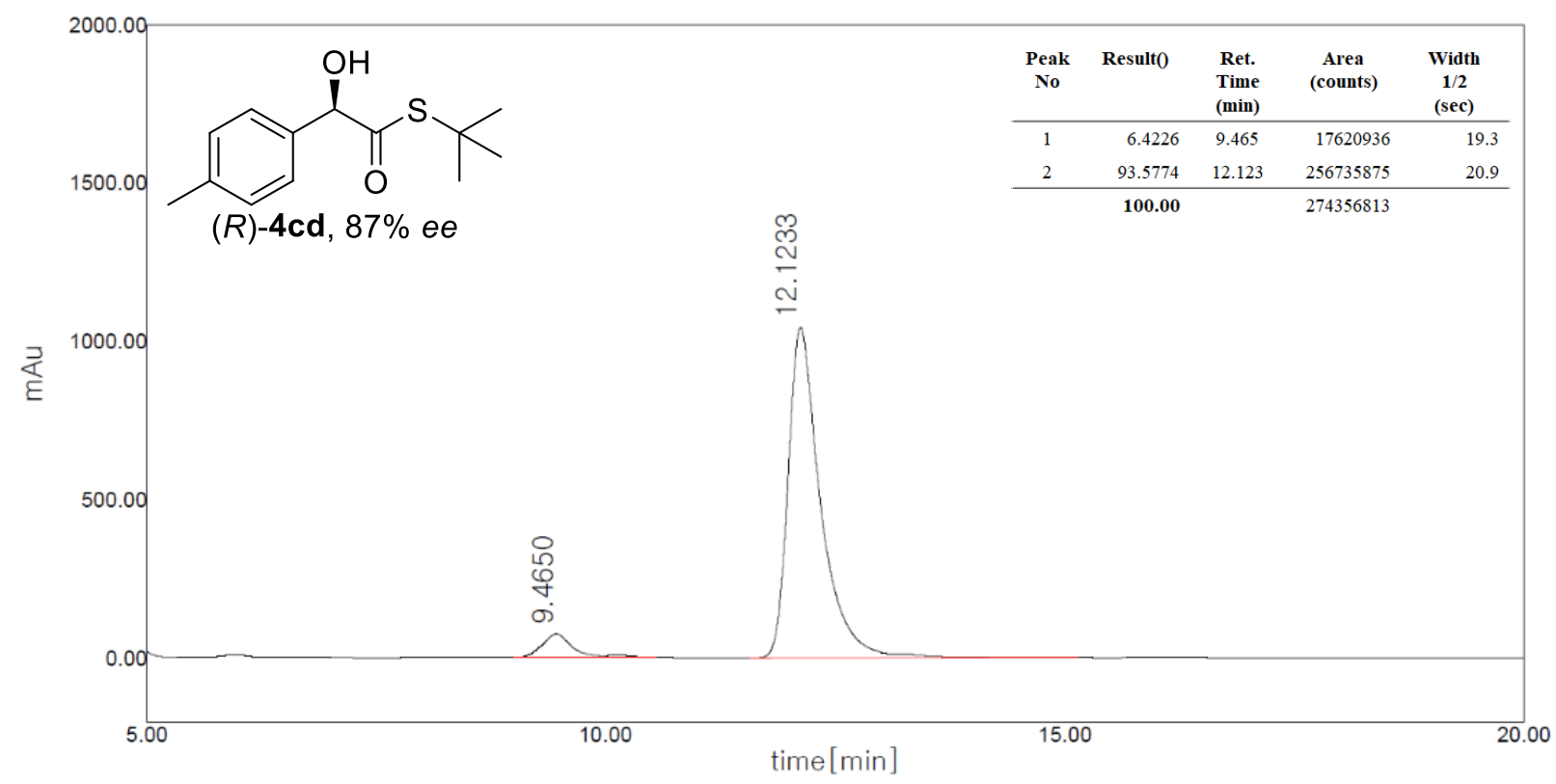

\section{HPLC trace of $\mathbf{4 c d}$ (condition b)}

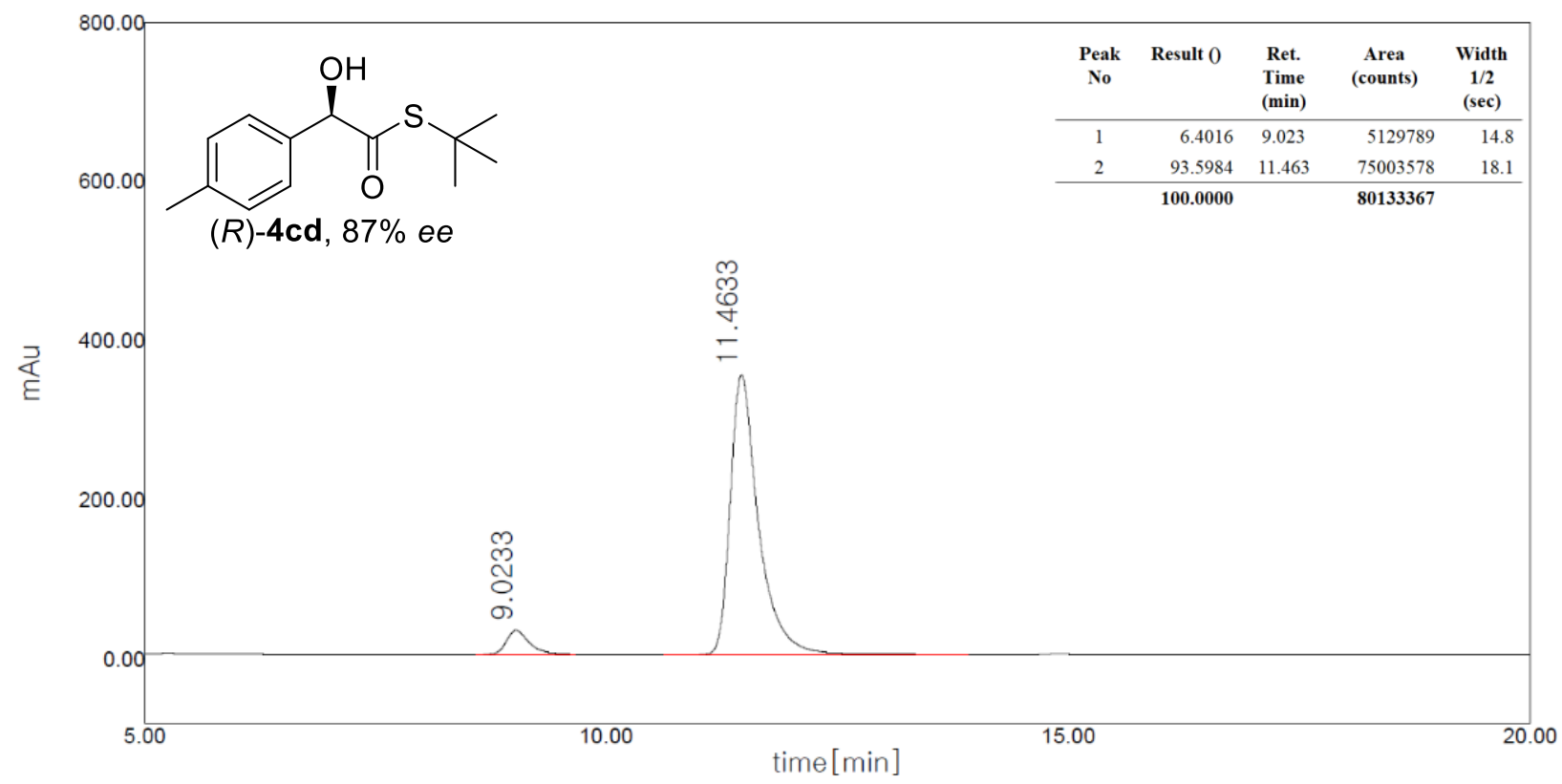

HPLC trace of $\mathbf{4 c d}$ (condition c) 


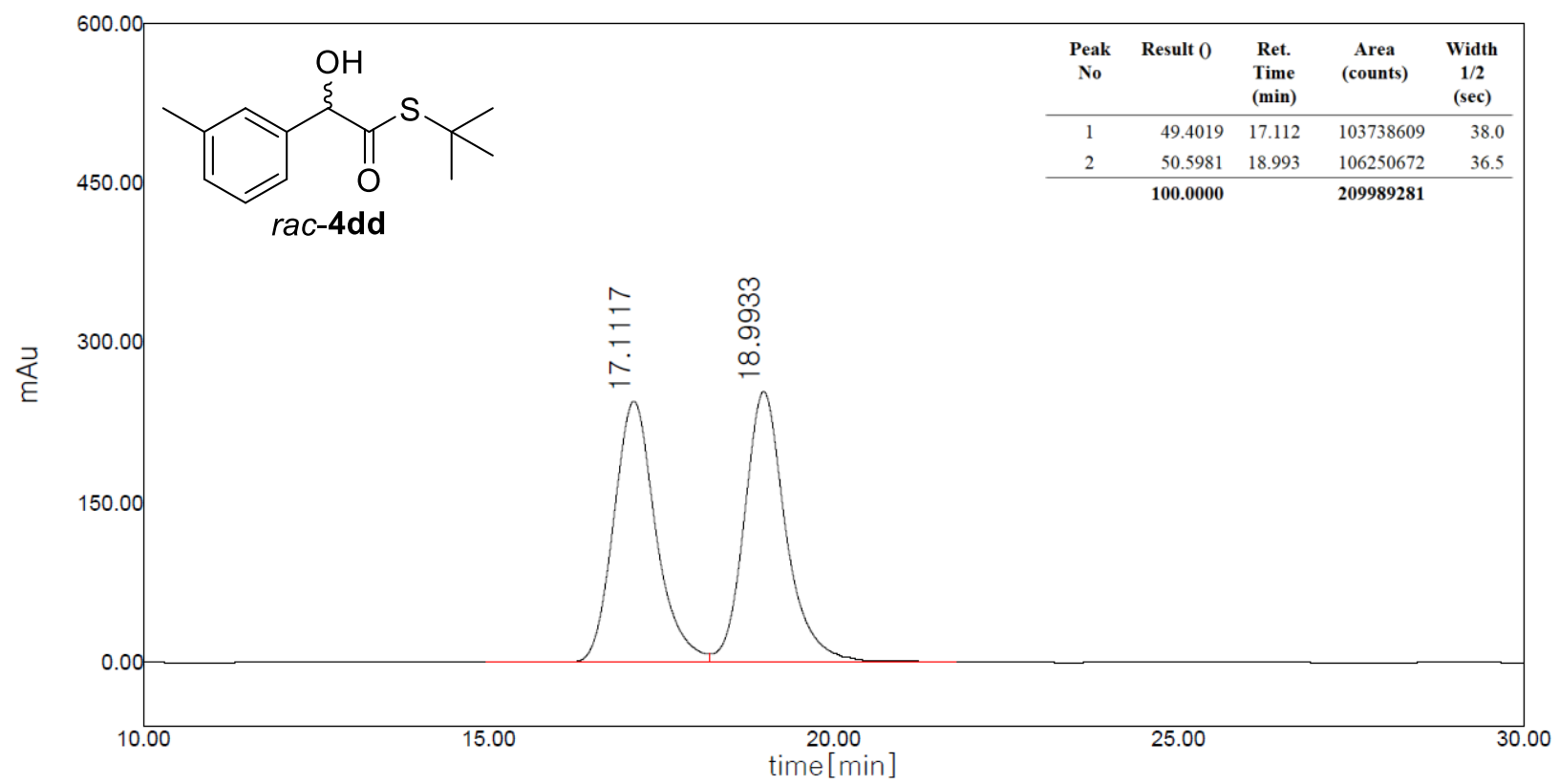

HPLC trace of $r a c-\mathbf{4 d d}$

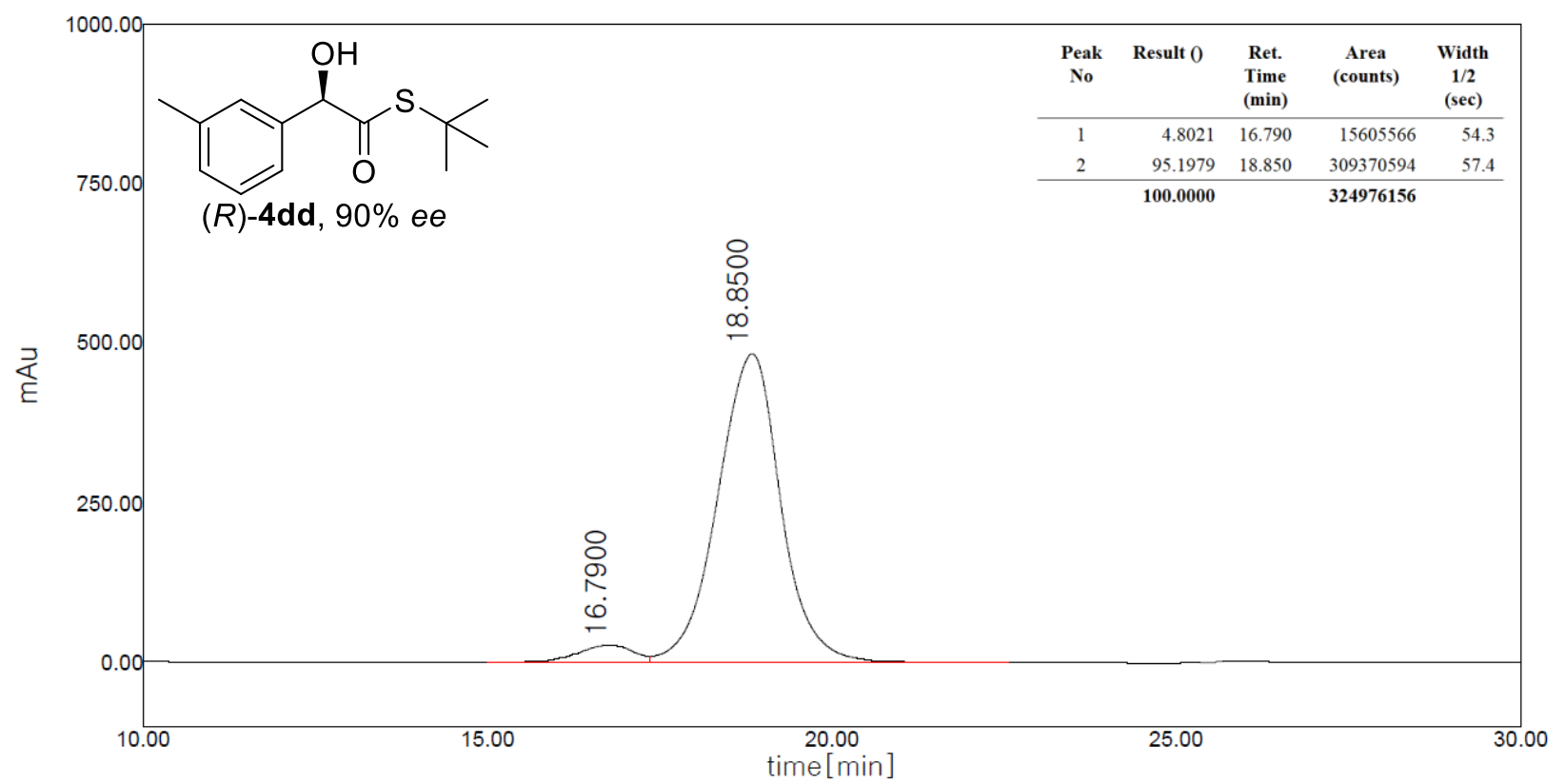

HPLC trace of 4dd (condition a) 


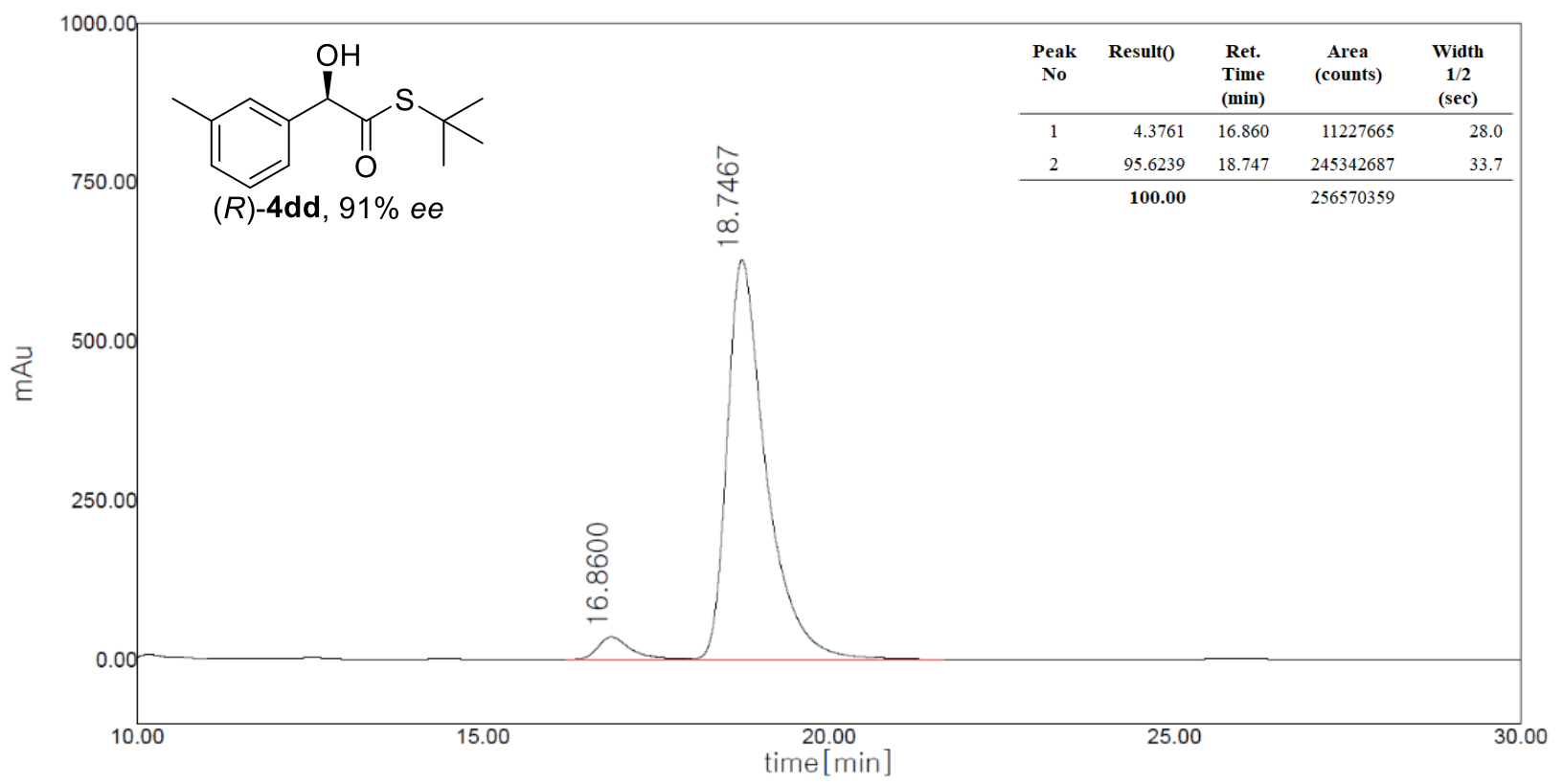

HPLC trace of 4dd (condition b)

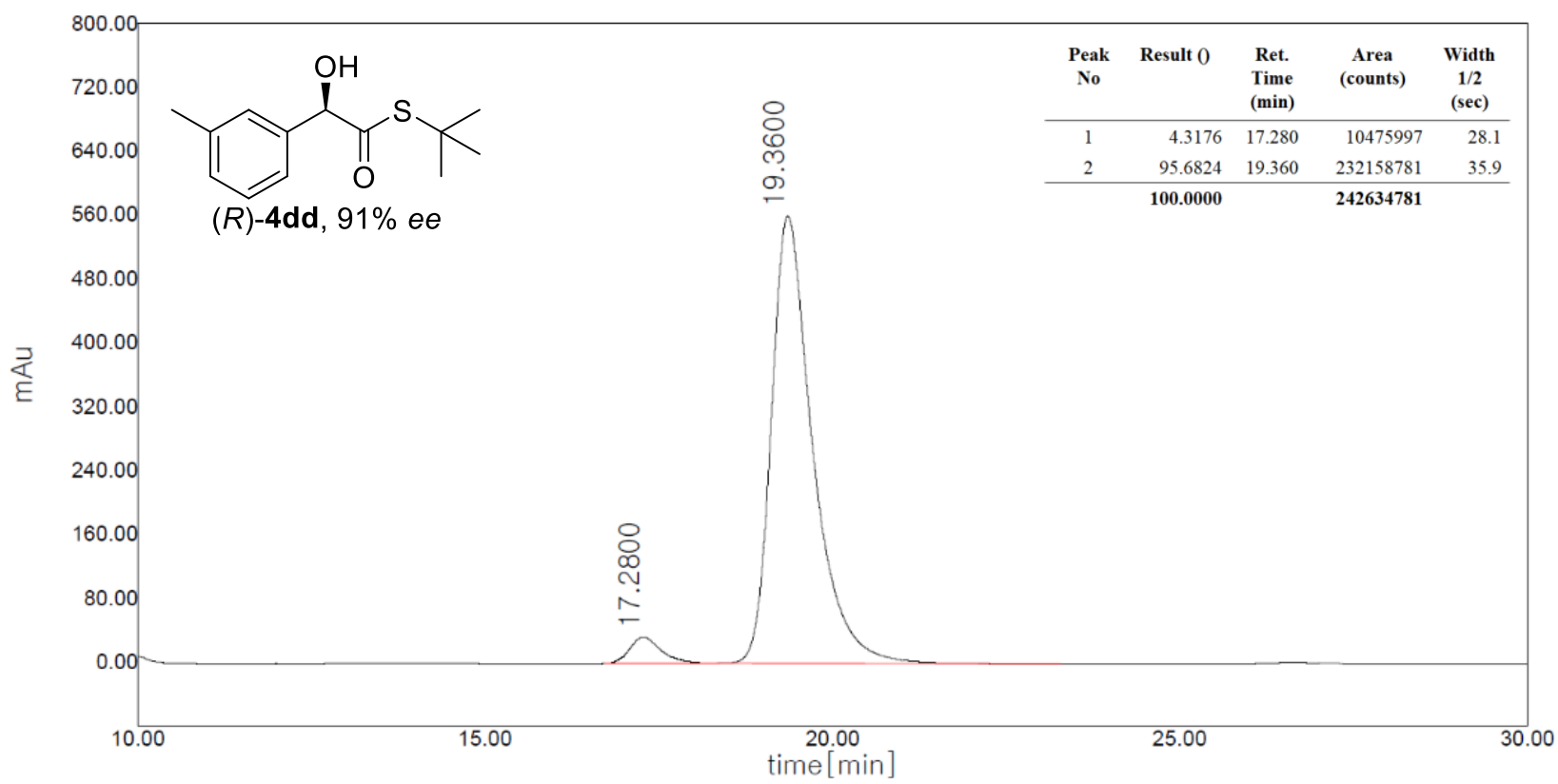

HPLC trace of 4dd (condition c) 


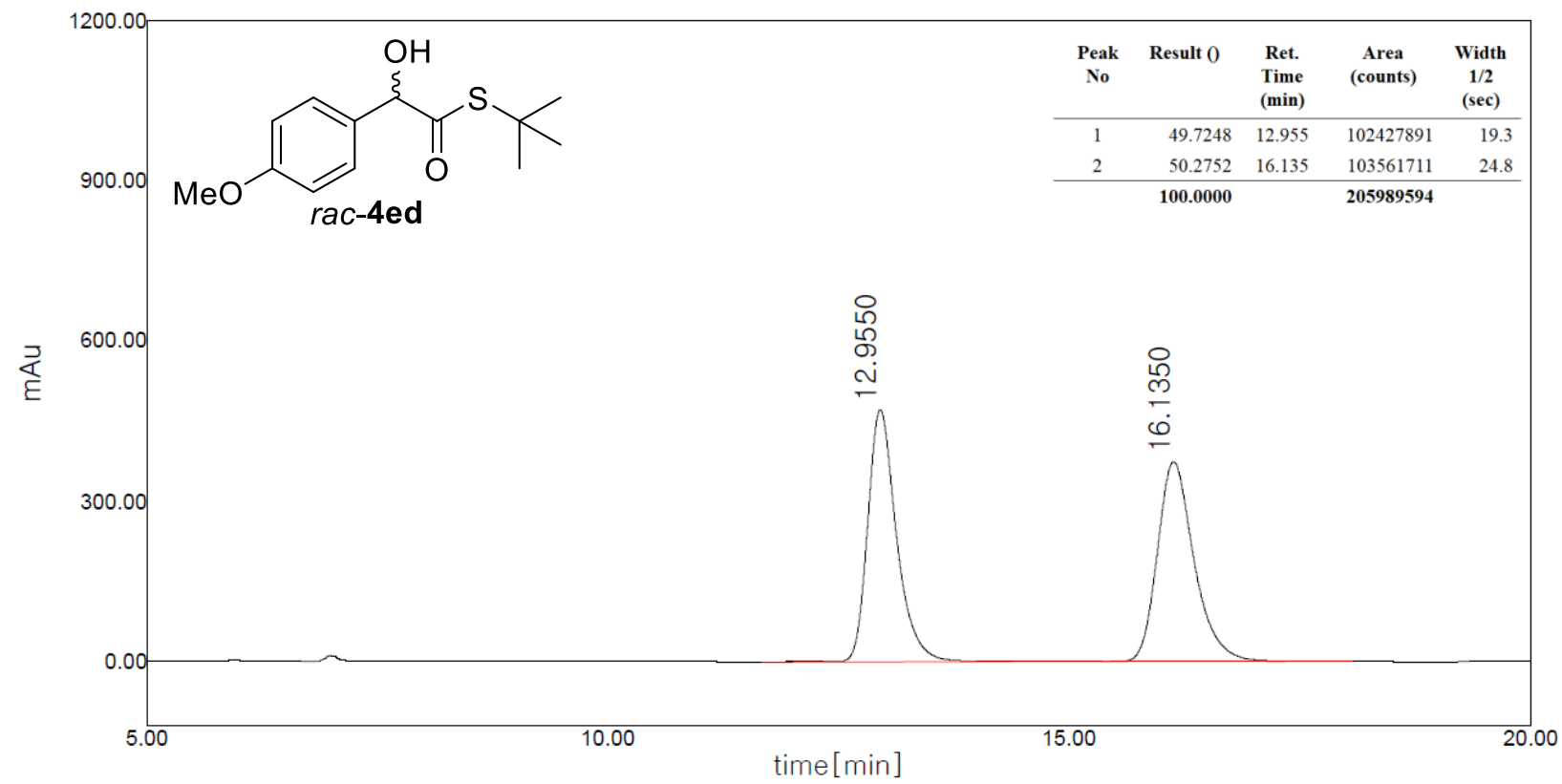

HPLC trace of $r a c-4 e d$

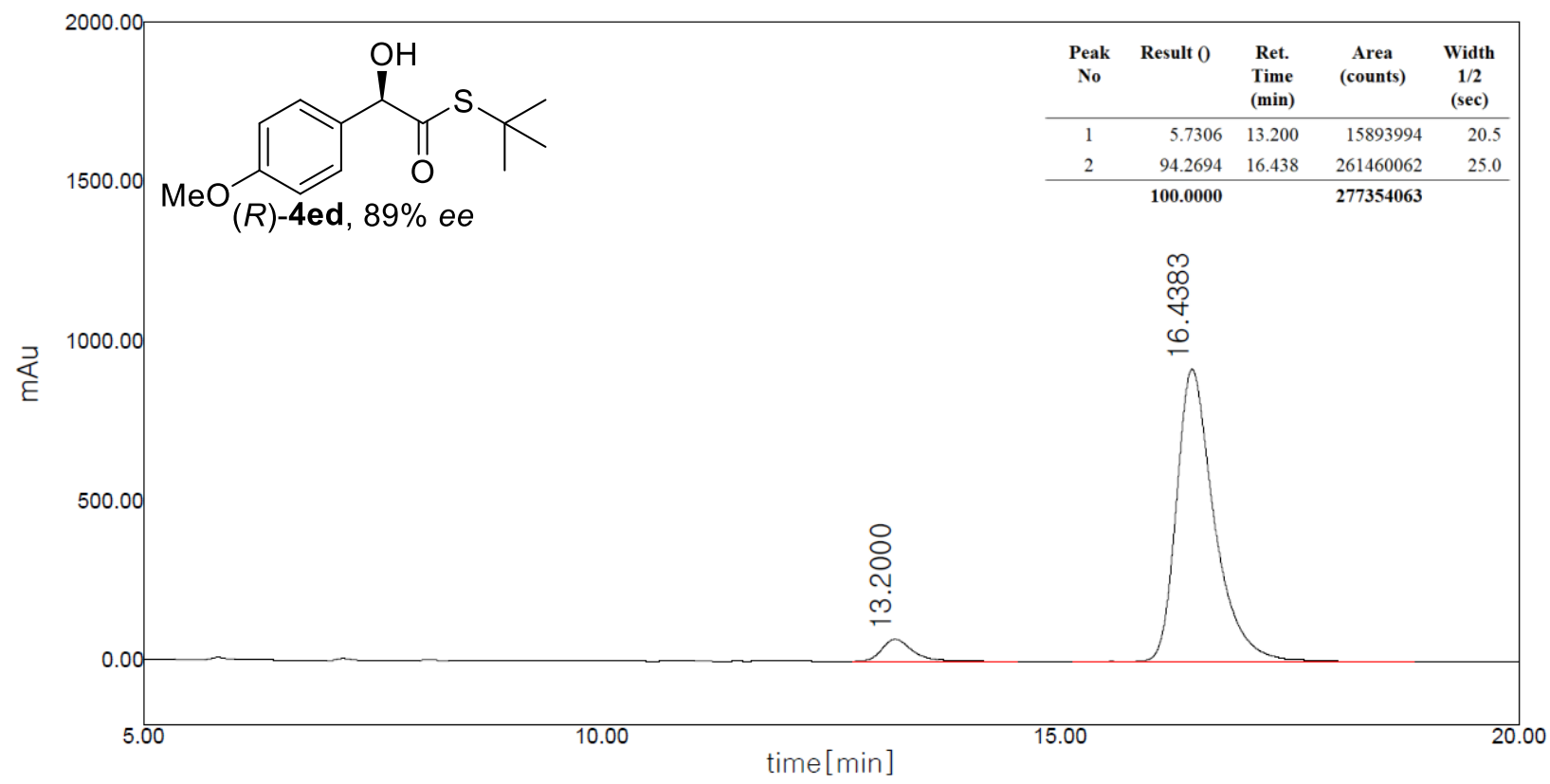

HPLC trace of 4ed (condition a) 


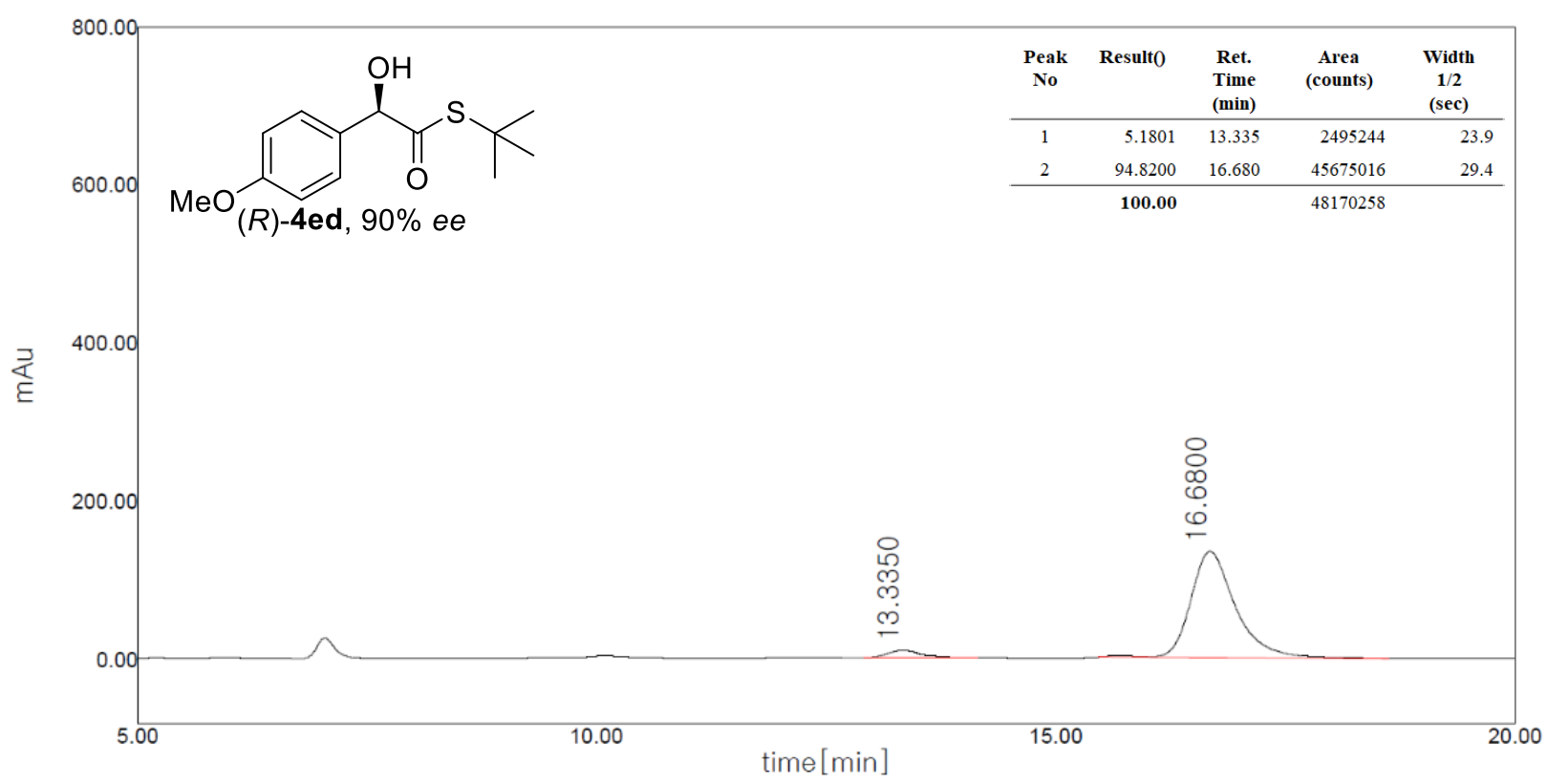

HPLC trace of 4ed (condition b)

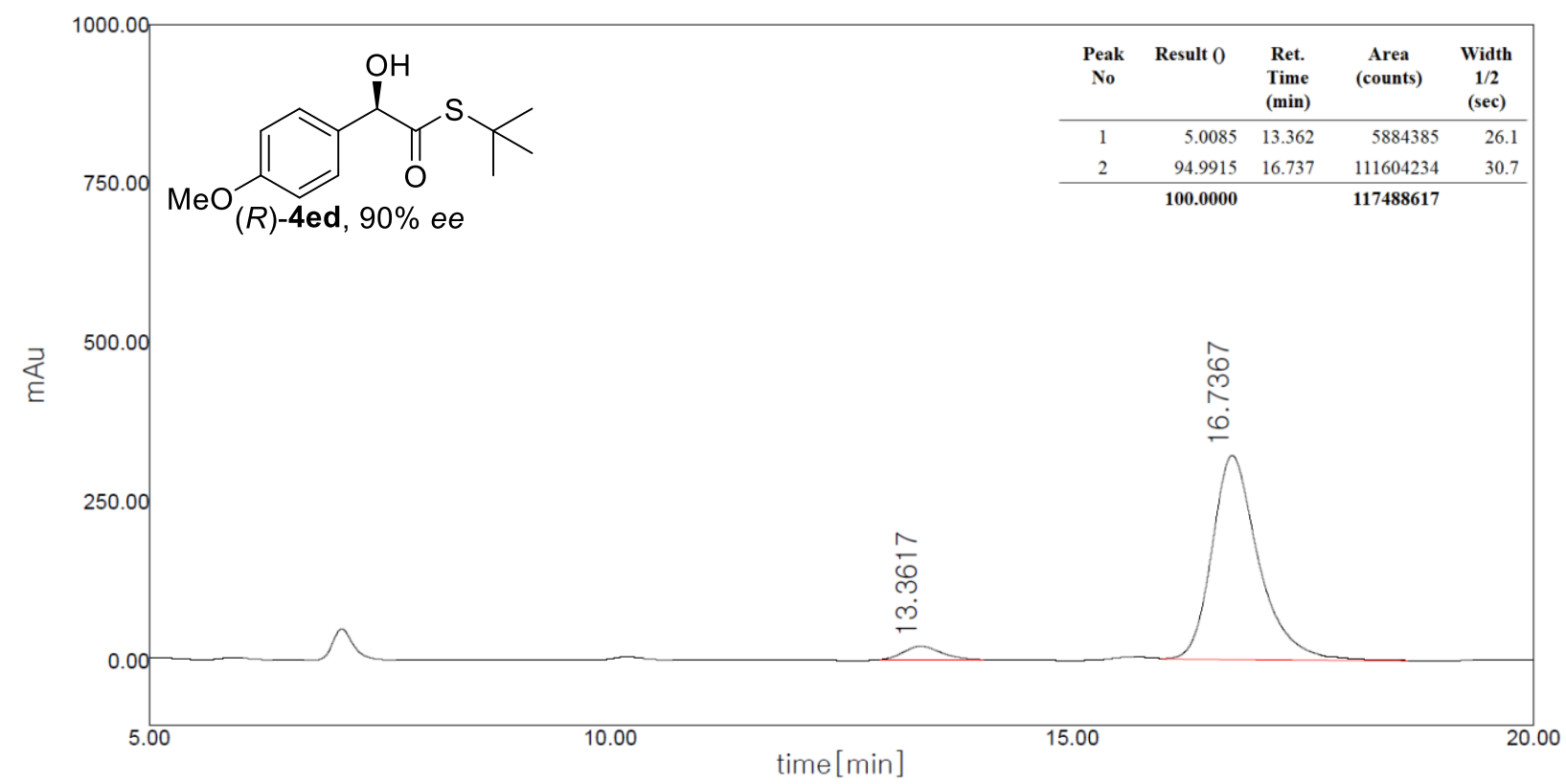

HPLC trace of 4ed (condition c) 


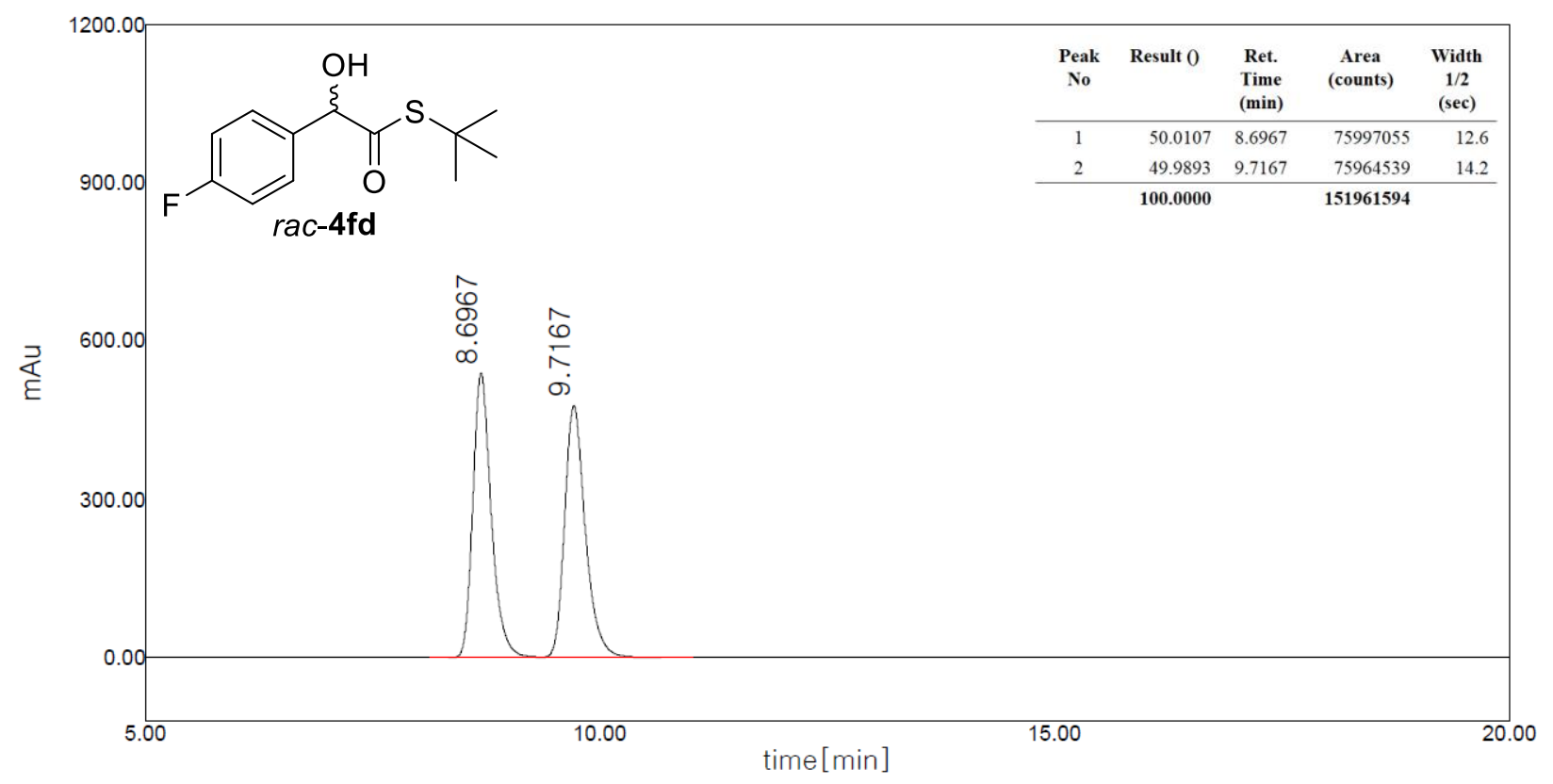

HPLC trace of $r a c-\mathbf{4 f d}$

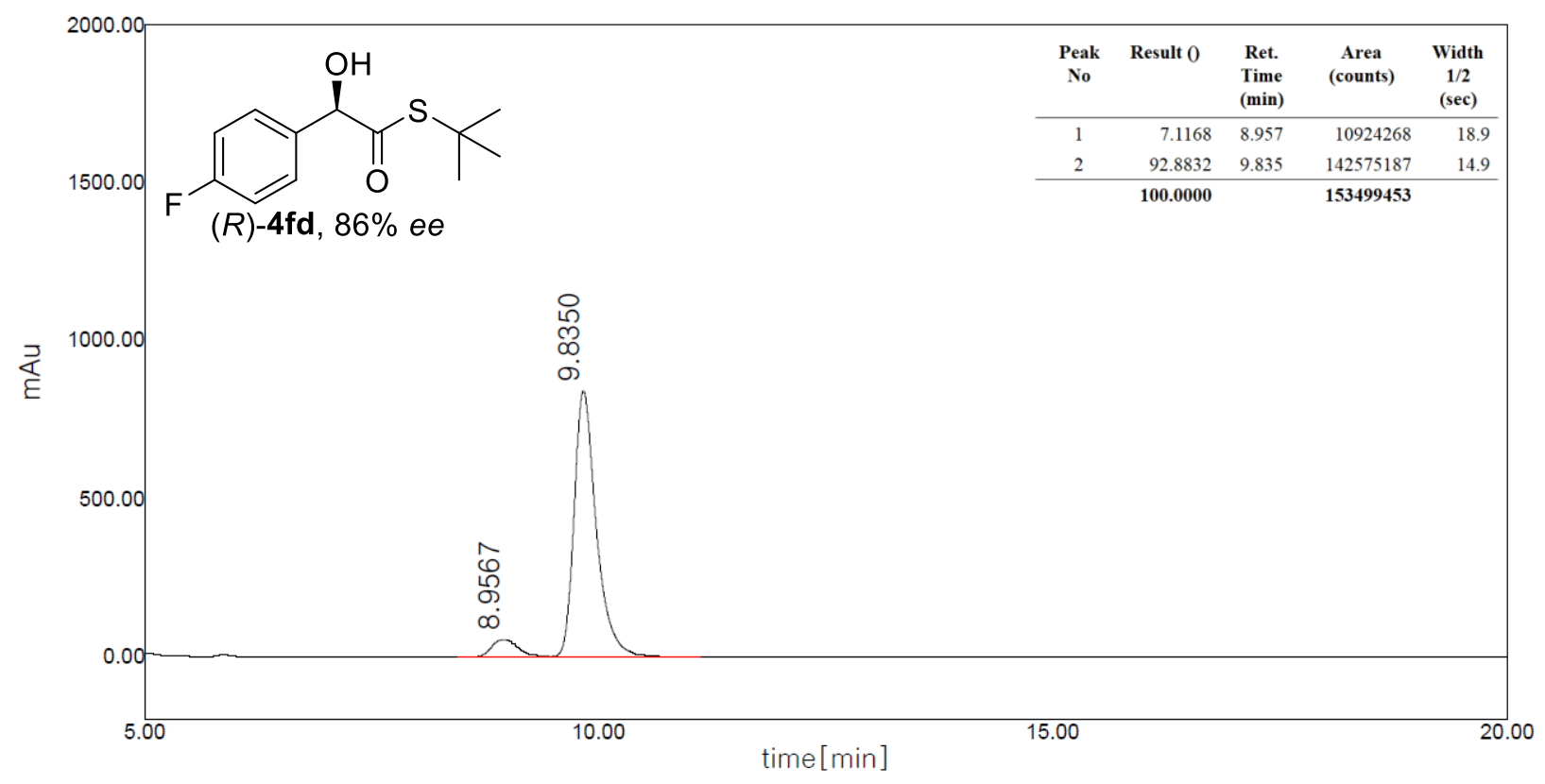

HPLC trace of $\mathbf{4 f d}$ (condition a) 


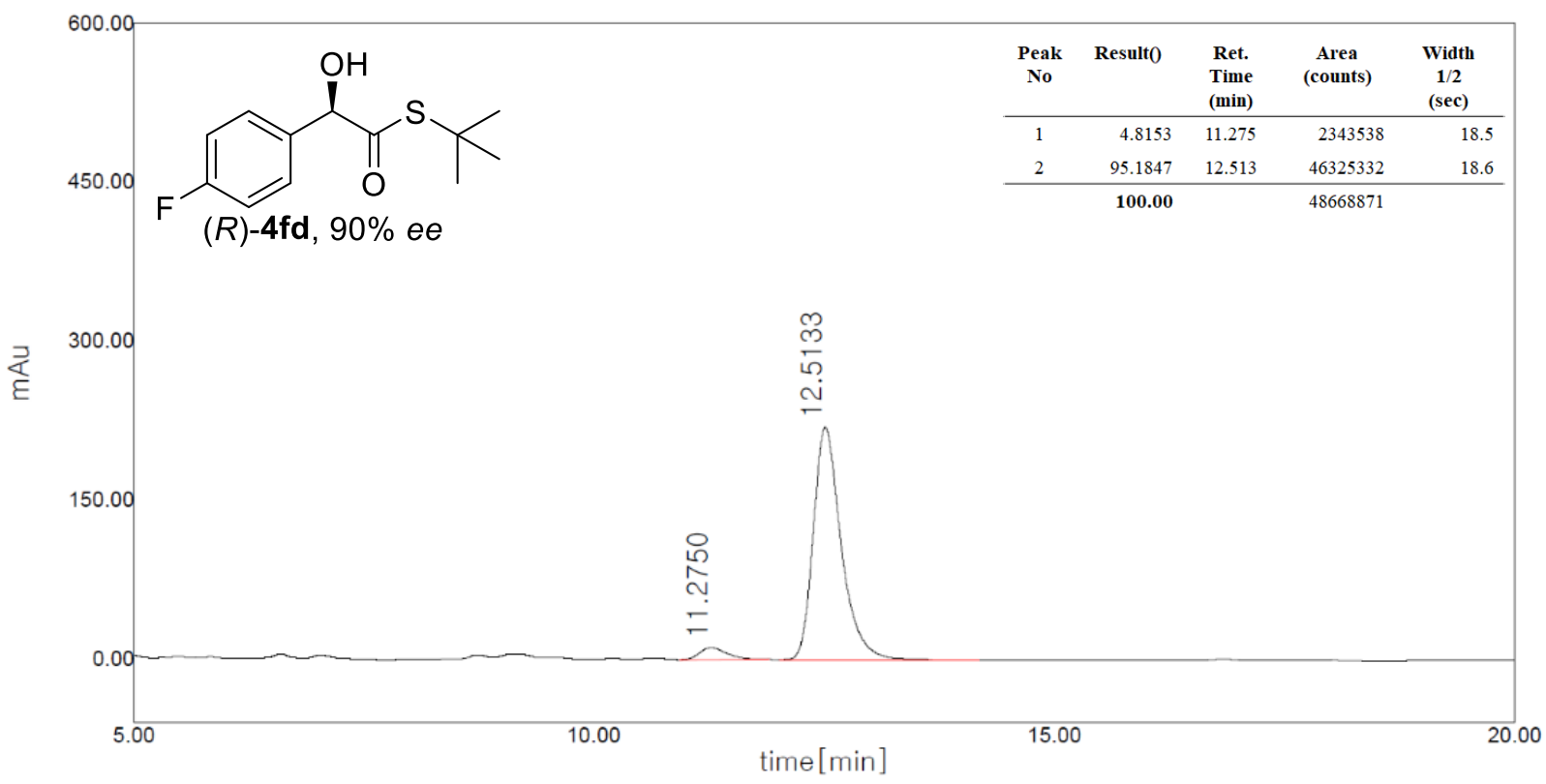

HPLC trace of $\mathbf{4 f d}$ (condition b)

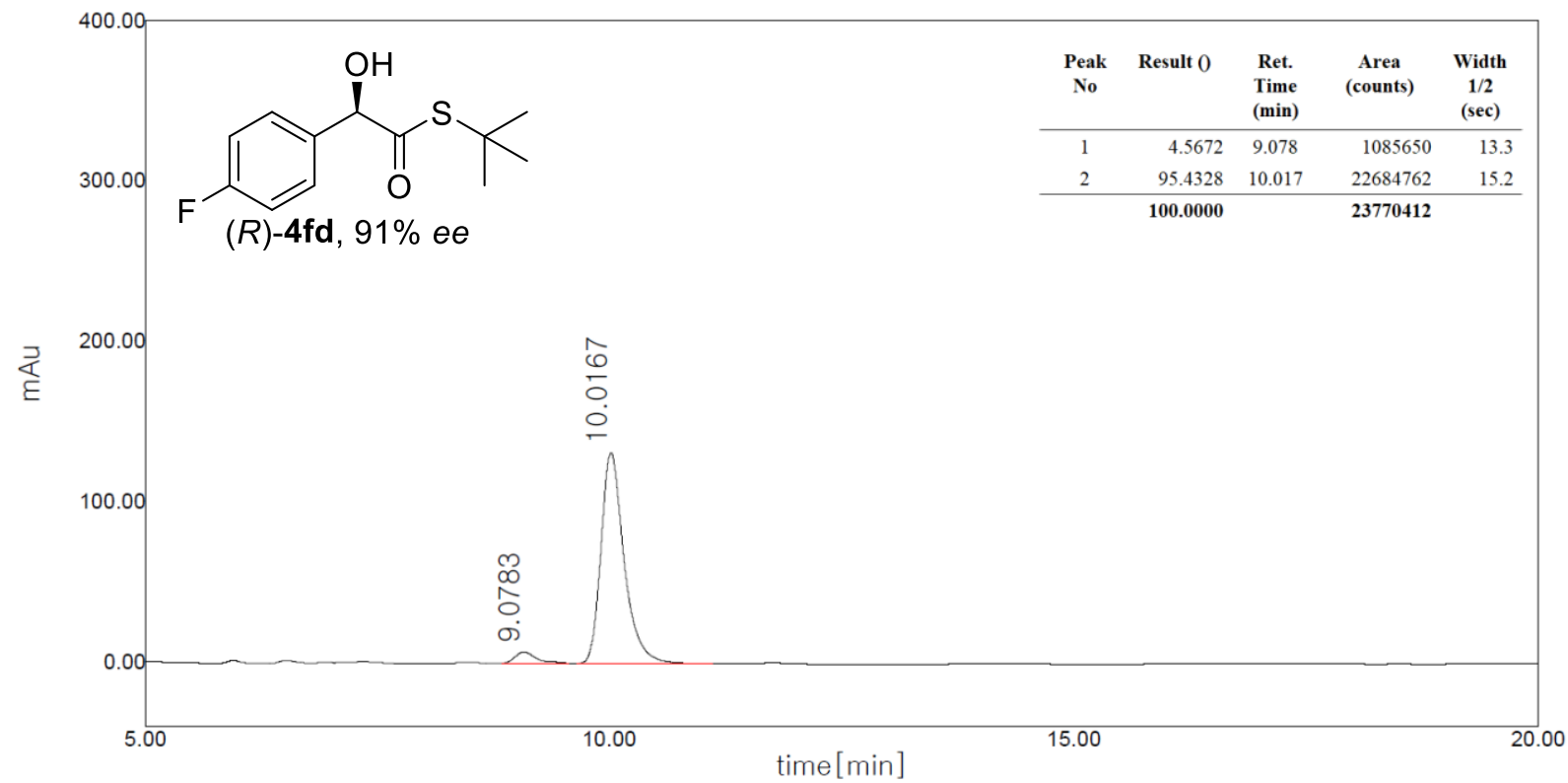

HPLC trace of $\mathbf{4 f d}$ (condition c) 


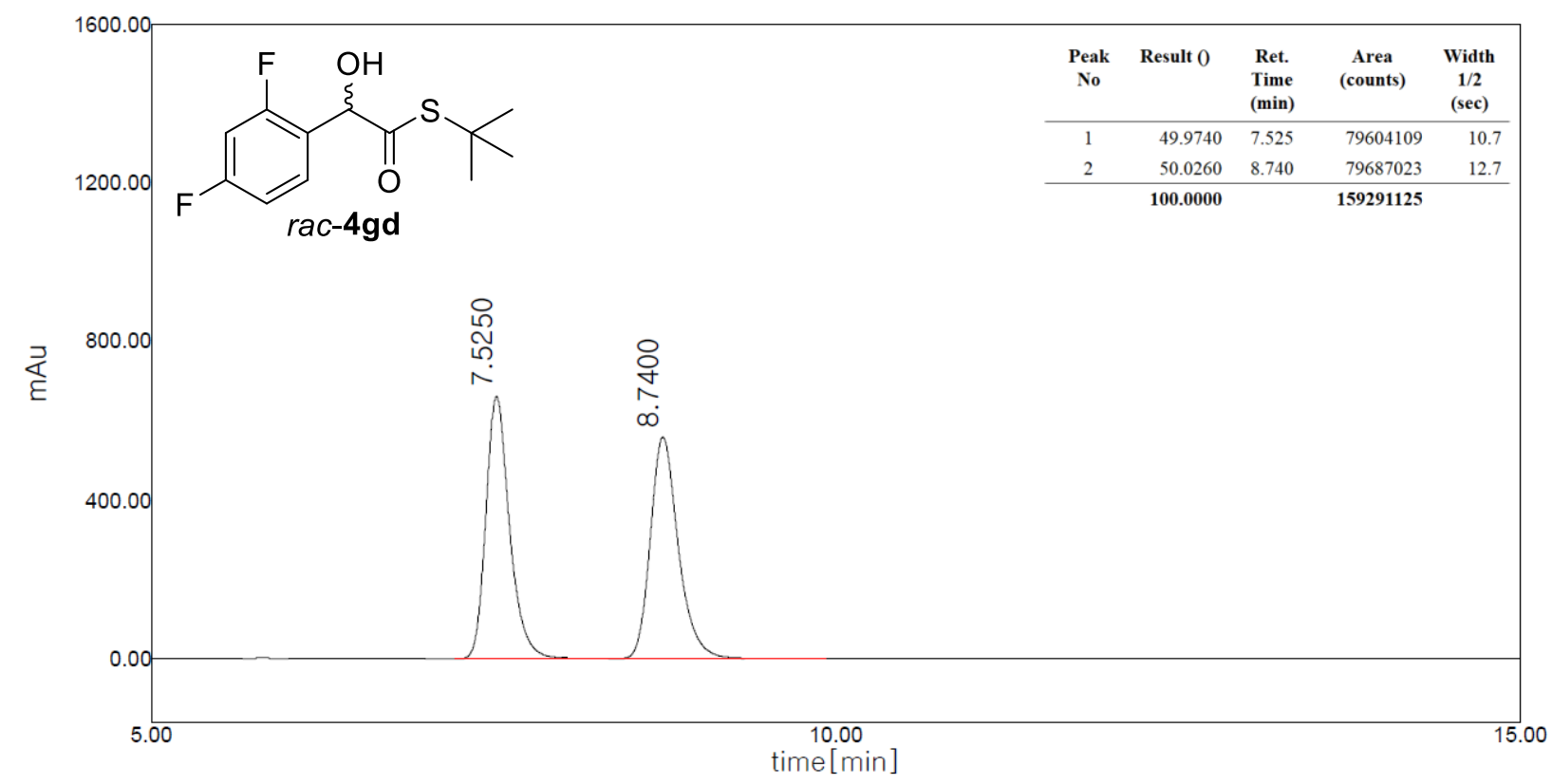

HPLC trace of $r a c-4 g d$

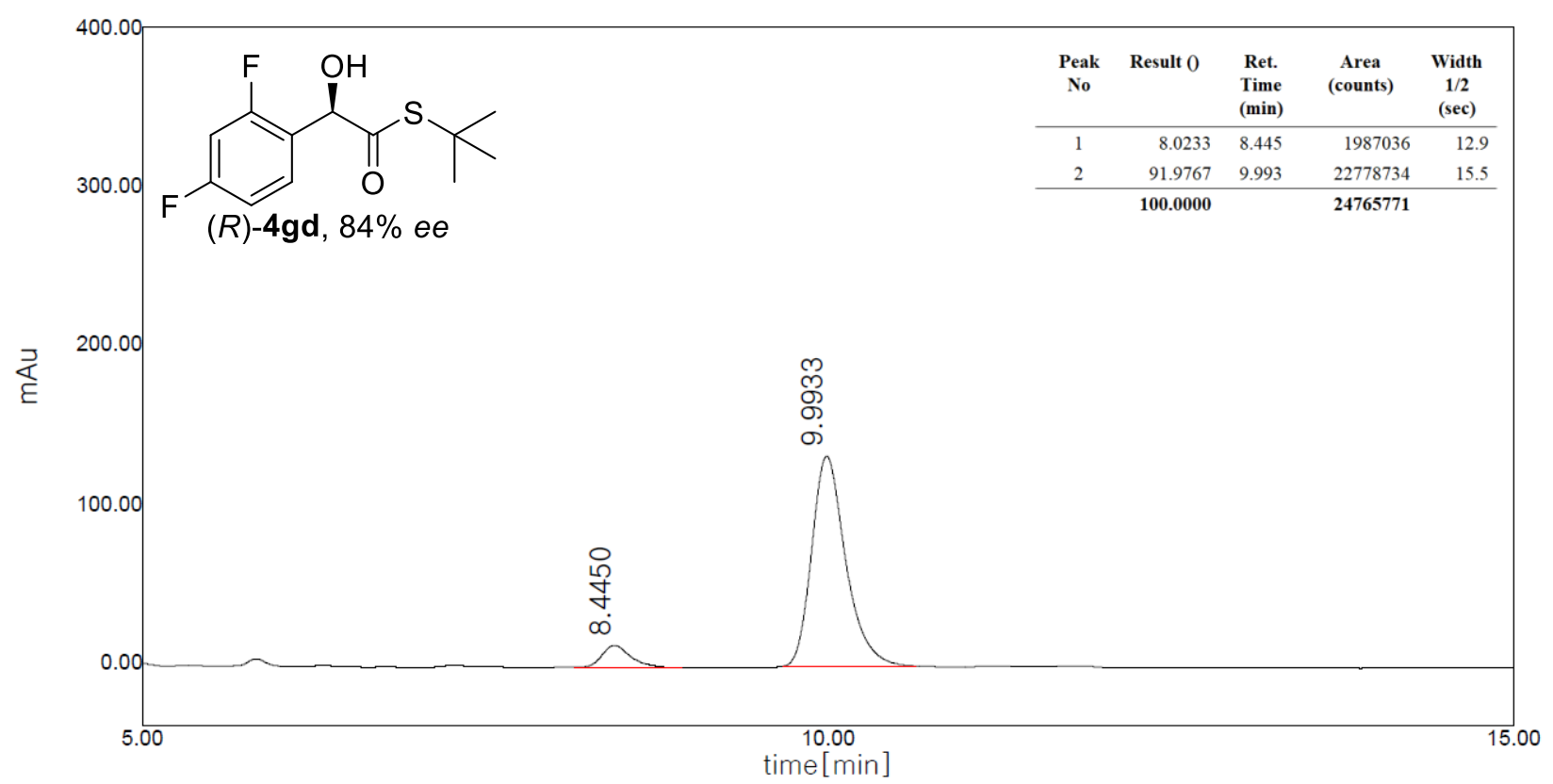

HPLC trace of 4gd (condition a) 


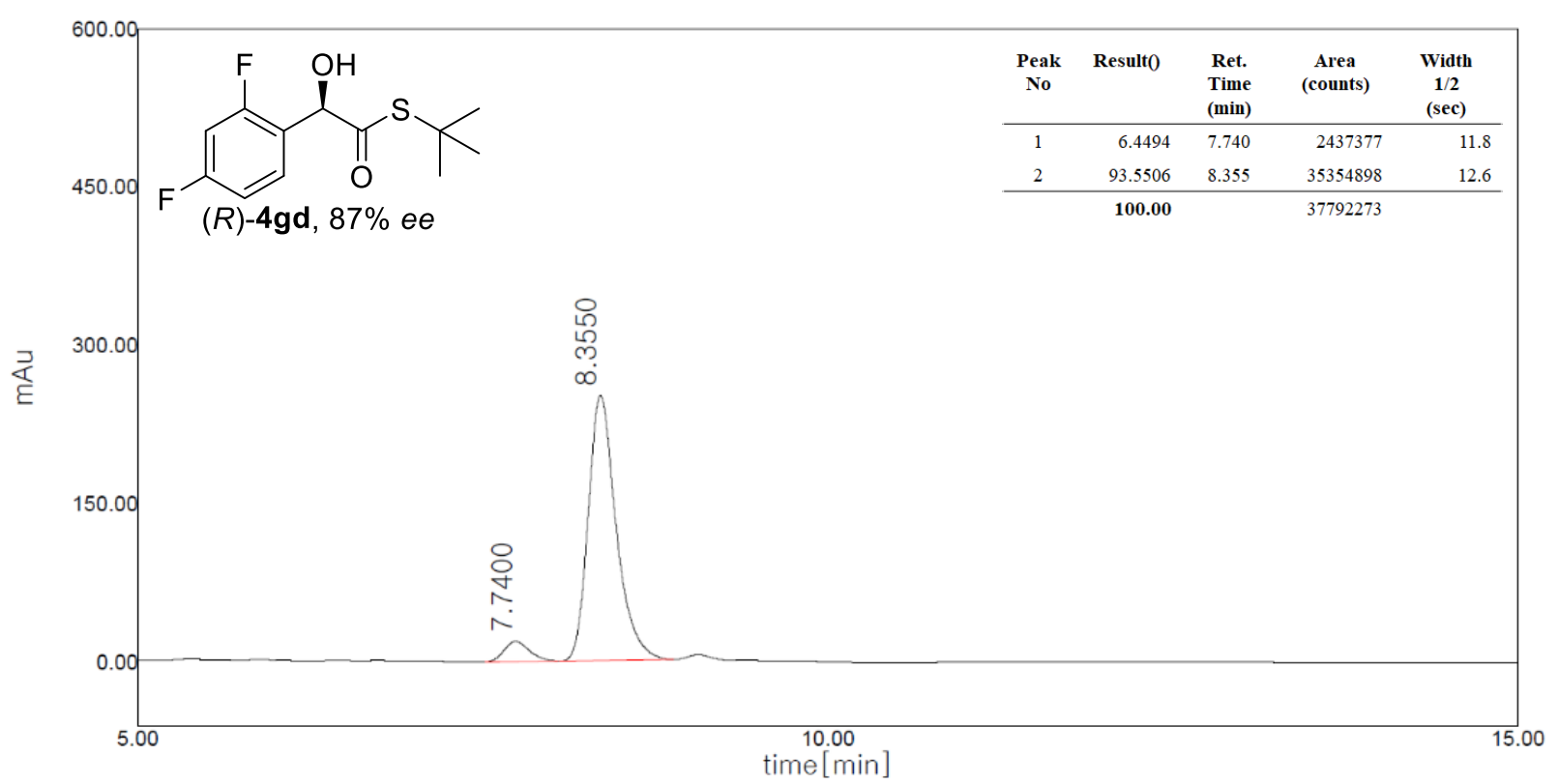

HPLC trace of 4gd (condition b)

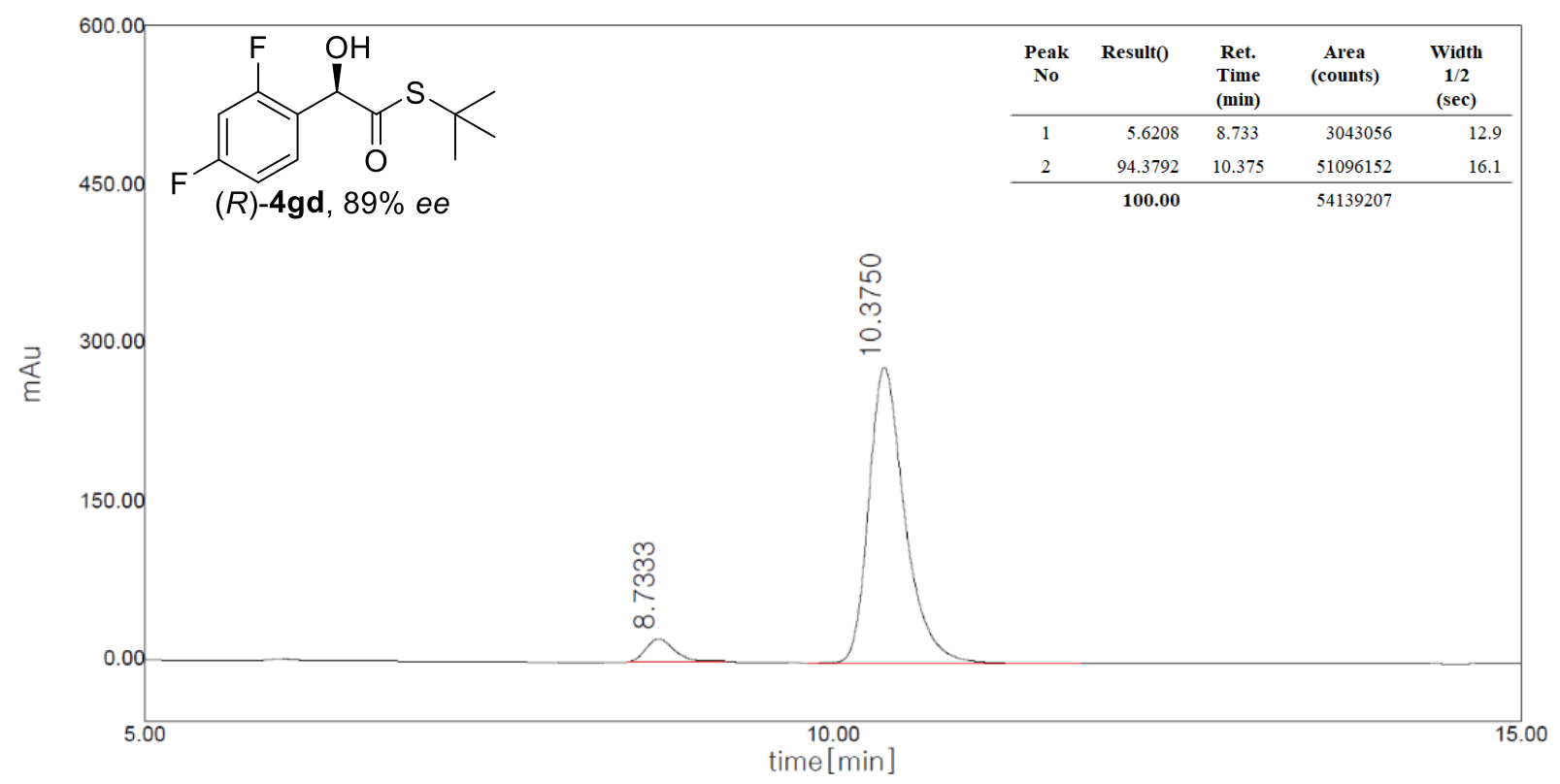

HPLC trace of 4gd (condition c) 


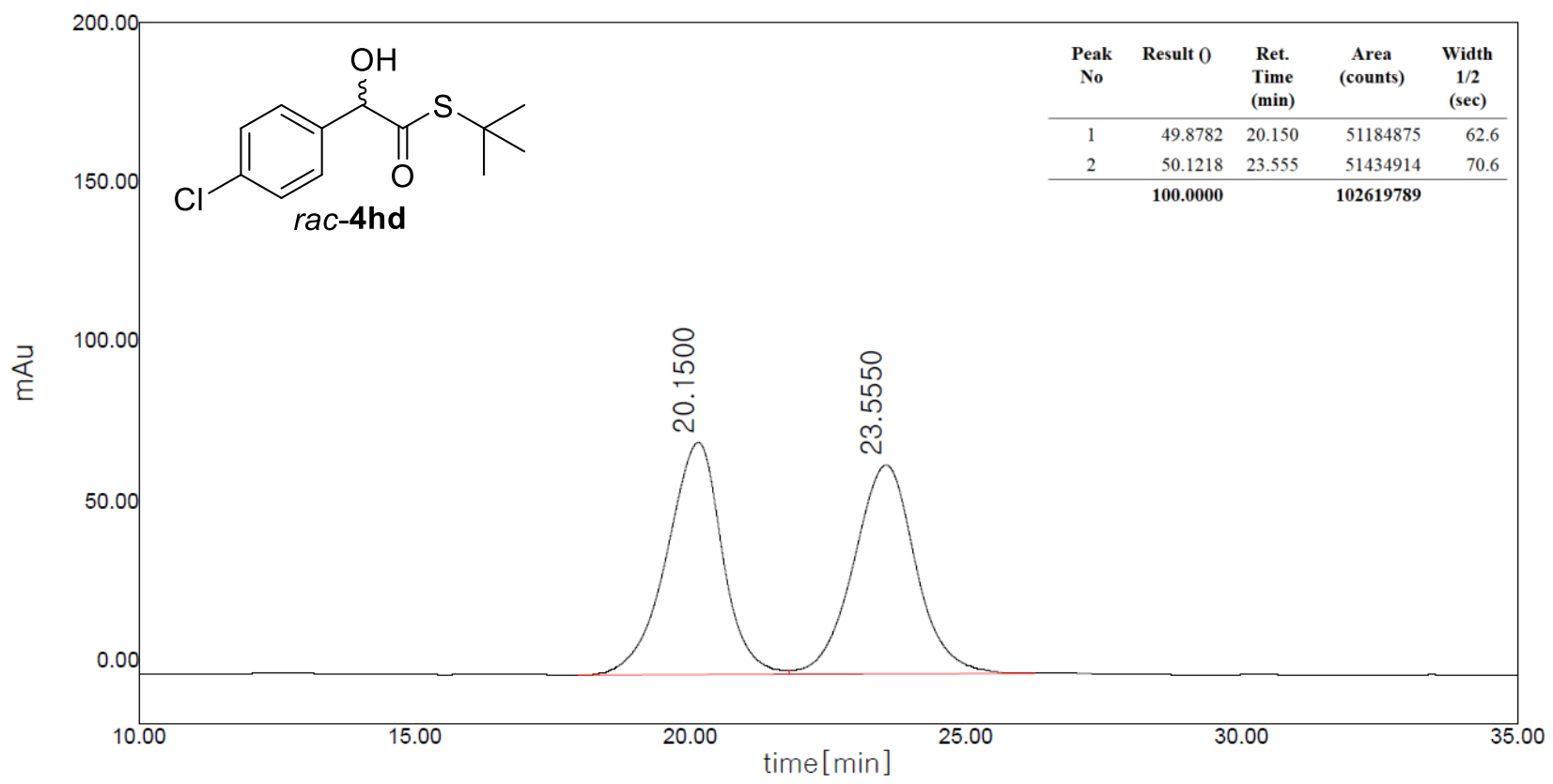

HPLC trace of $r a c-4 h d$

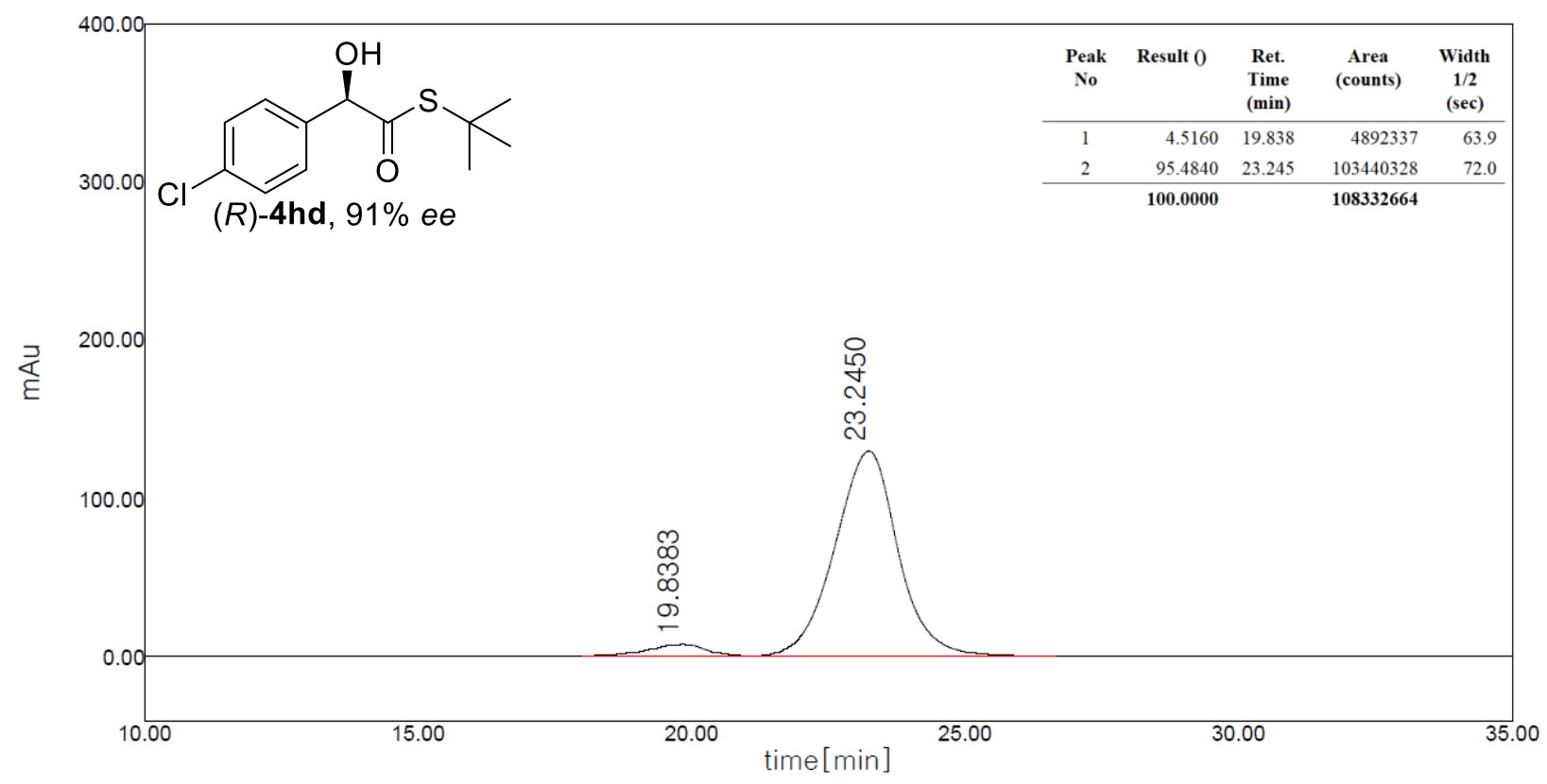

HPLC trace of $\mathbf{4 h d}$ (condition a) 


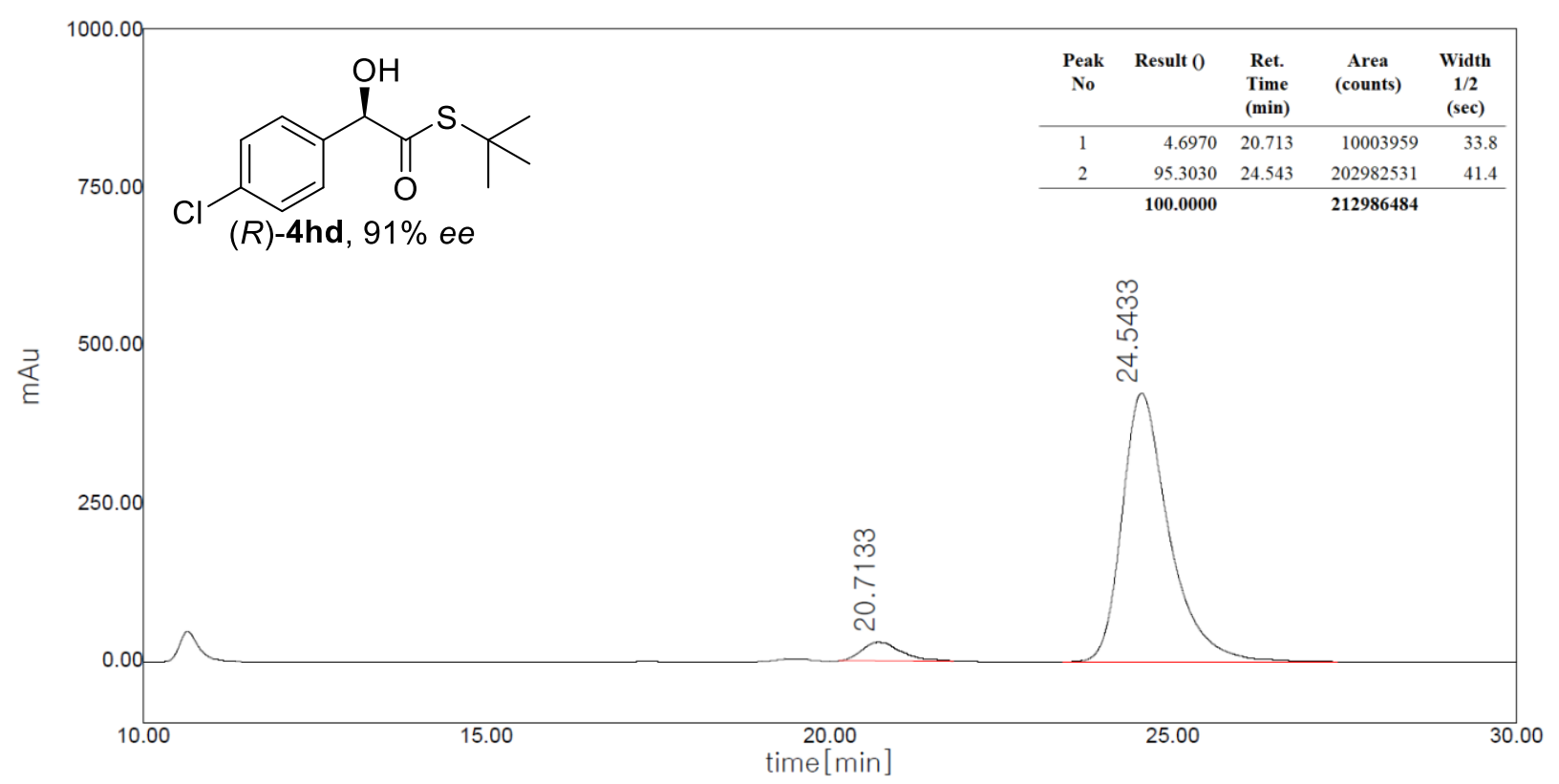

HPLC trace of $\mathbf{4 h d}$ (condition b)

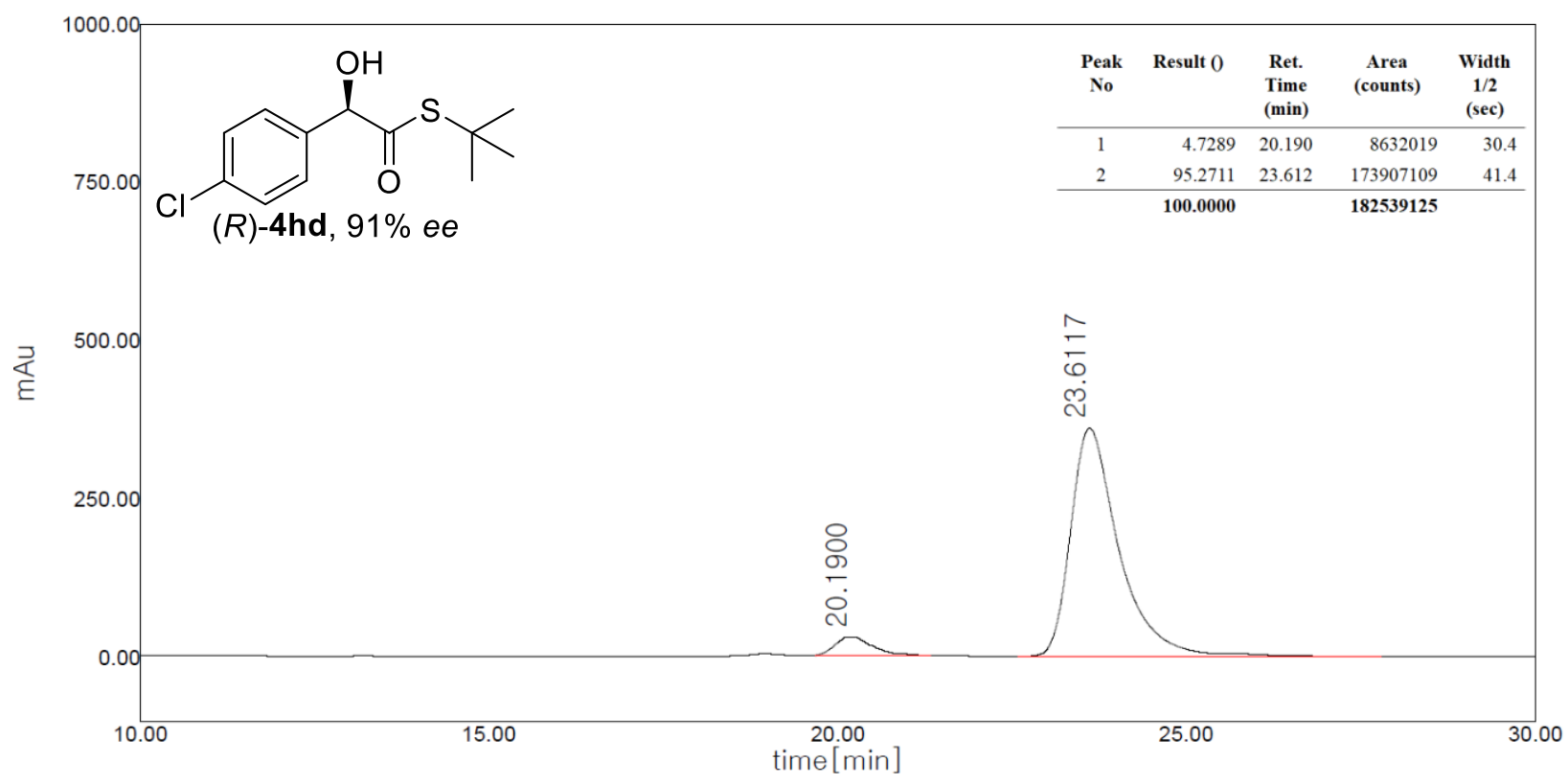

HPLC trace of $\mathbf{4 h d}$ (condition c) 


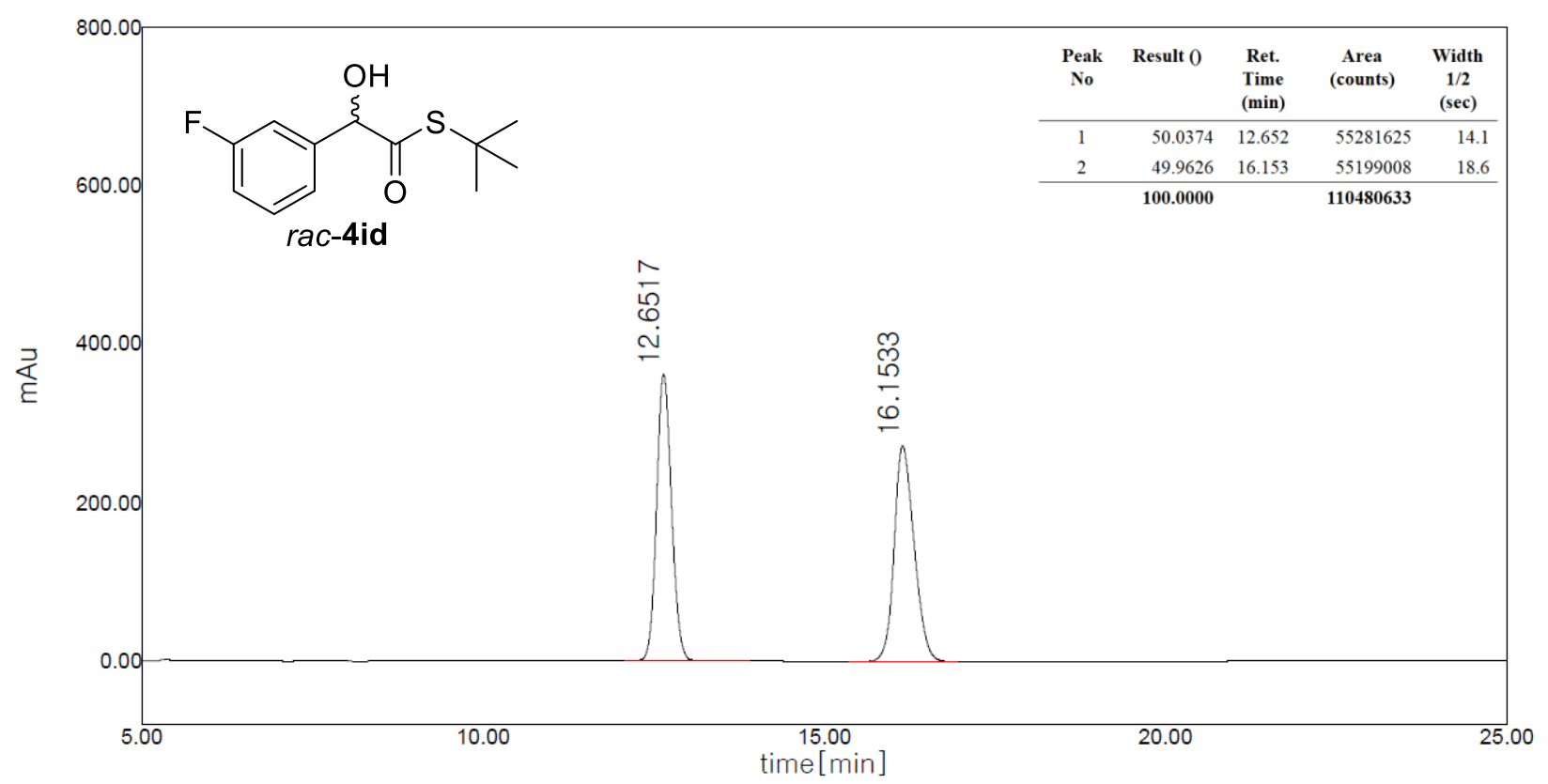

\section{HPLC trace of rac-4id}

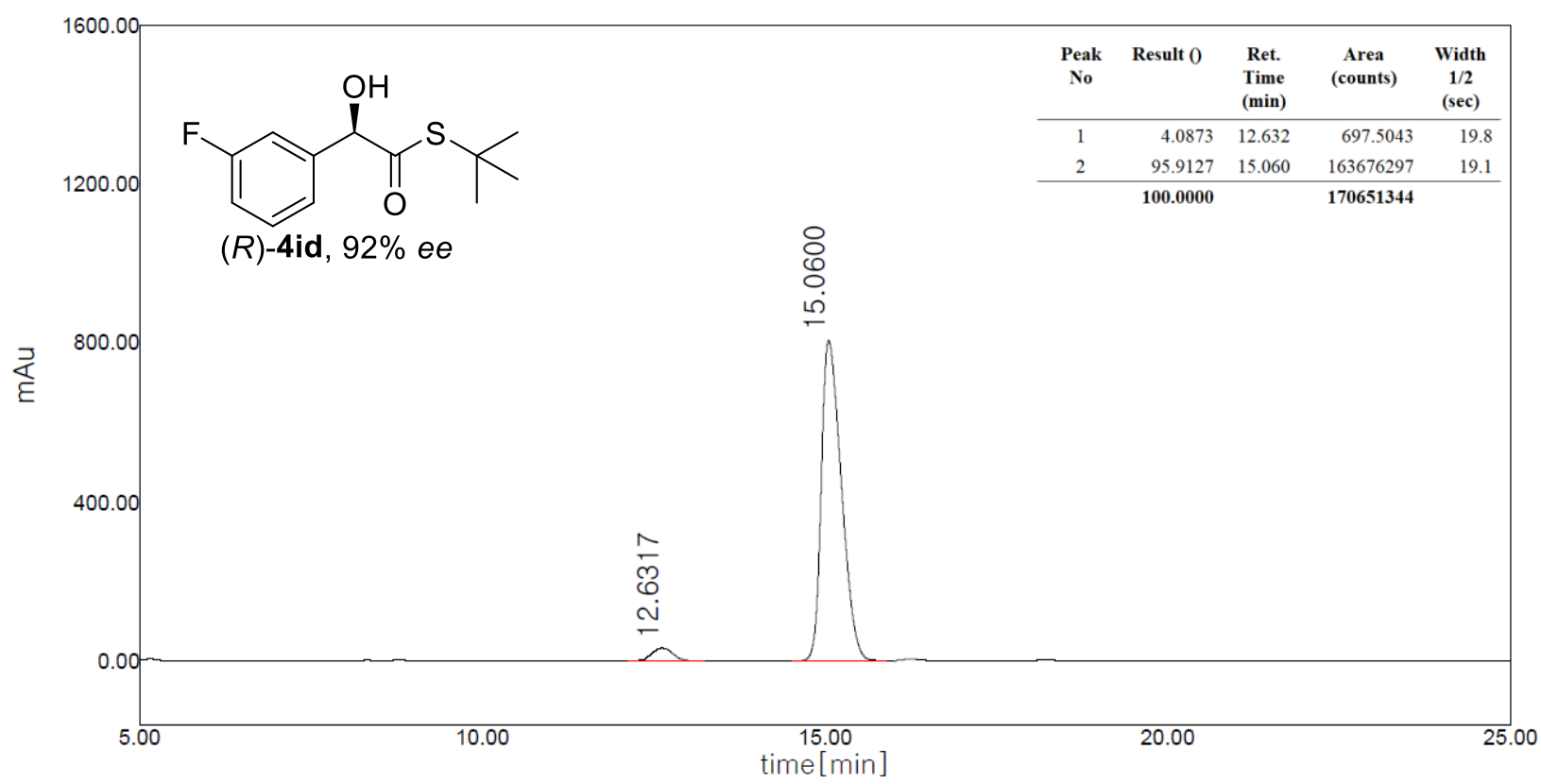

HPLC trace of 4id (condition a) 


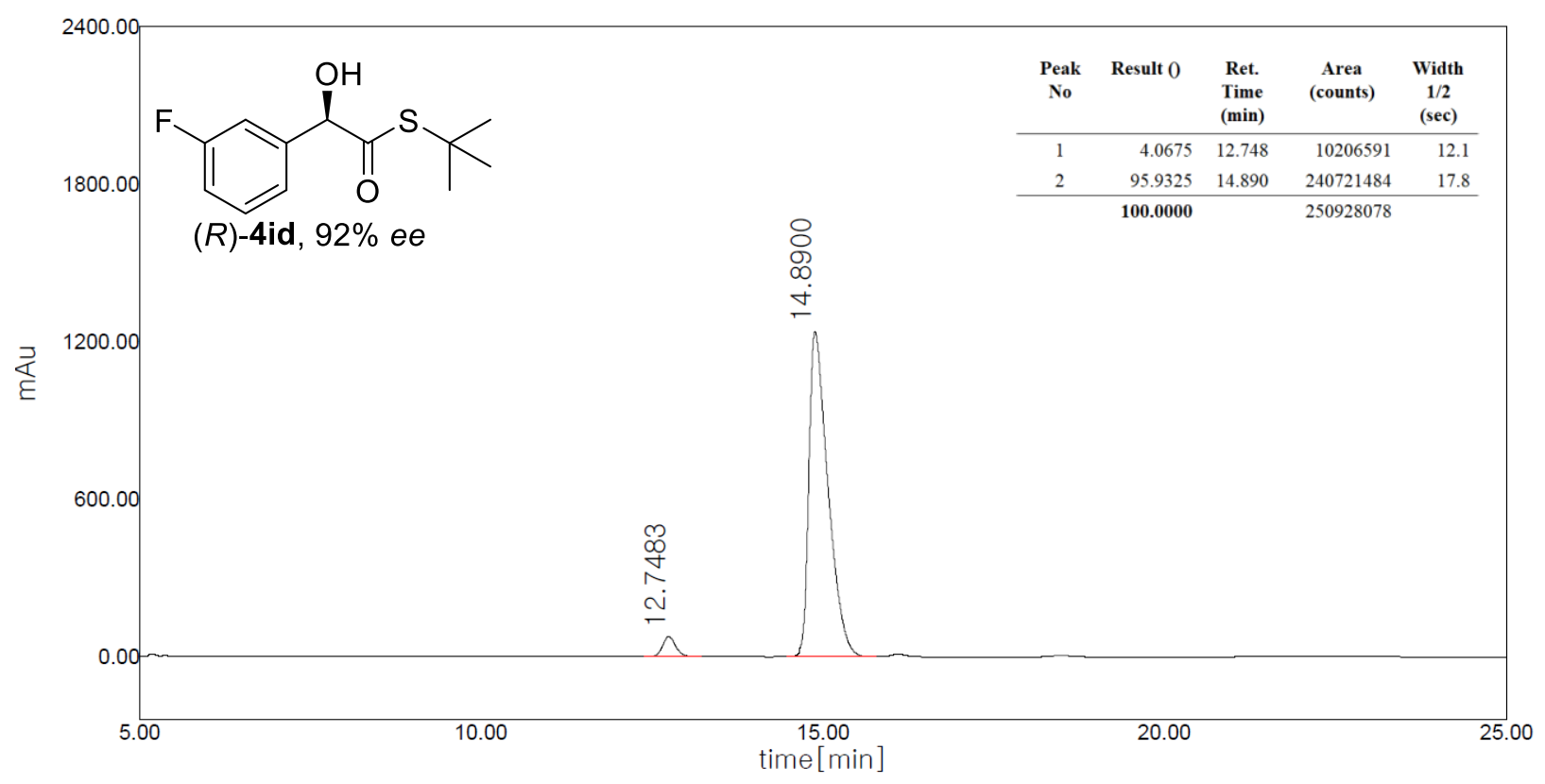

\section{HPLC trace of 4id (condition b)}

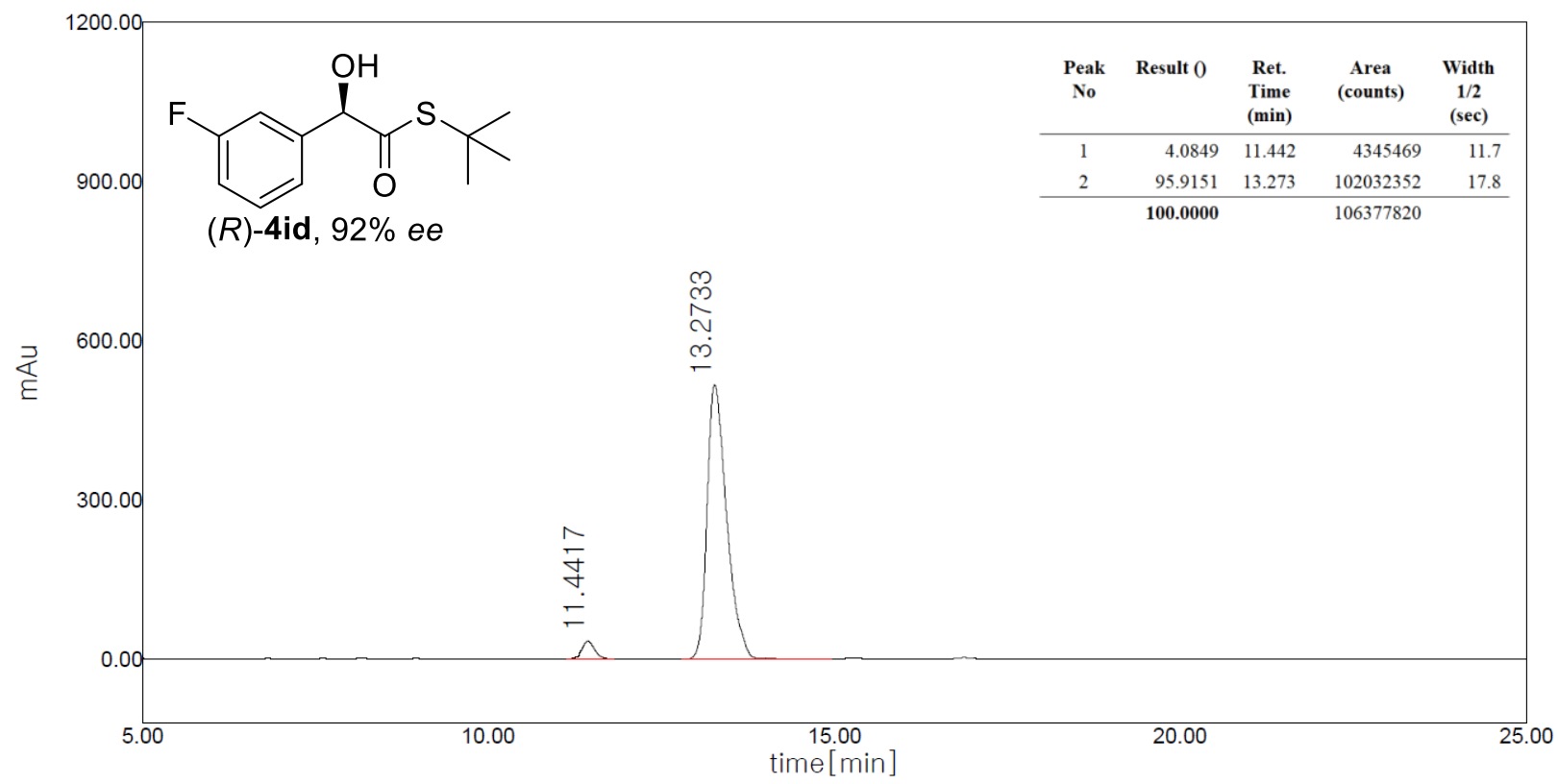

HPLC trace of 4id (condition c) 


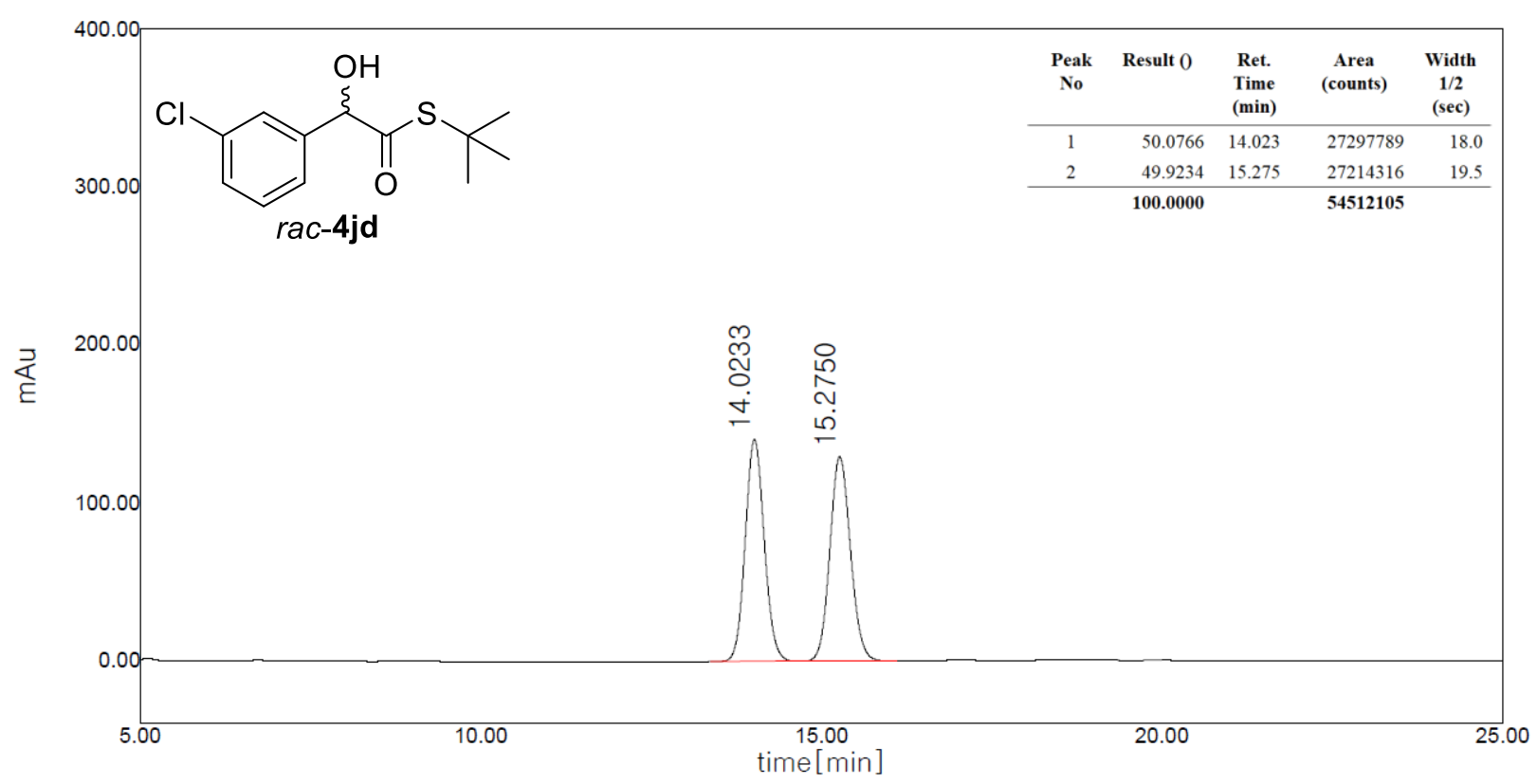

HPLC trace of rac-4jd

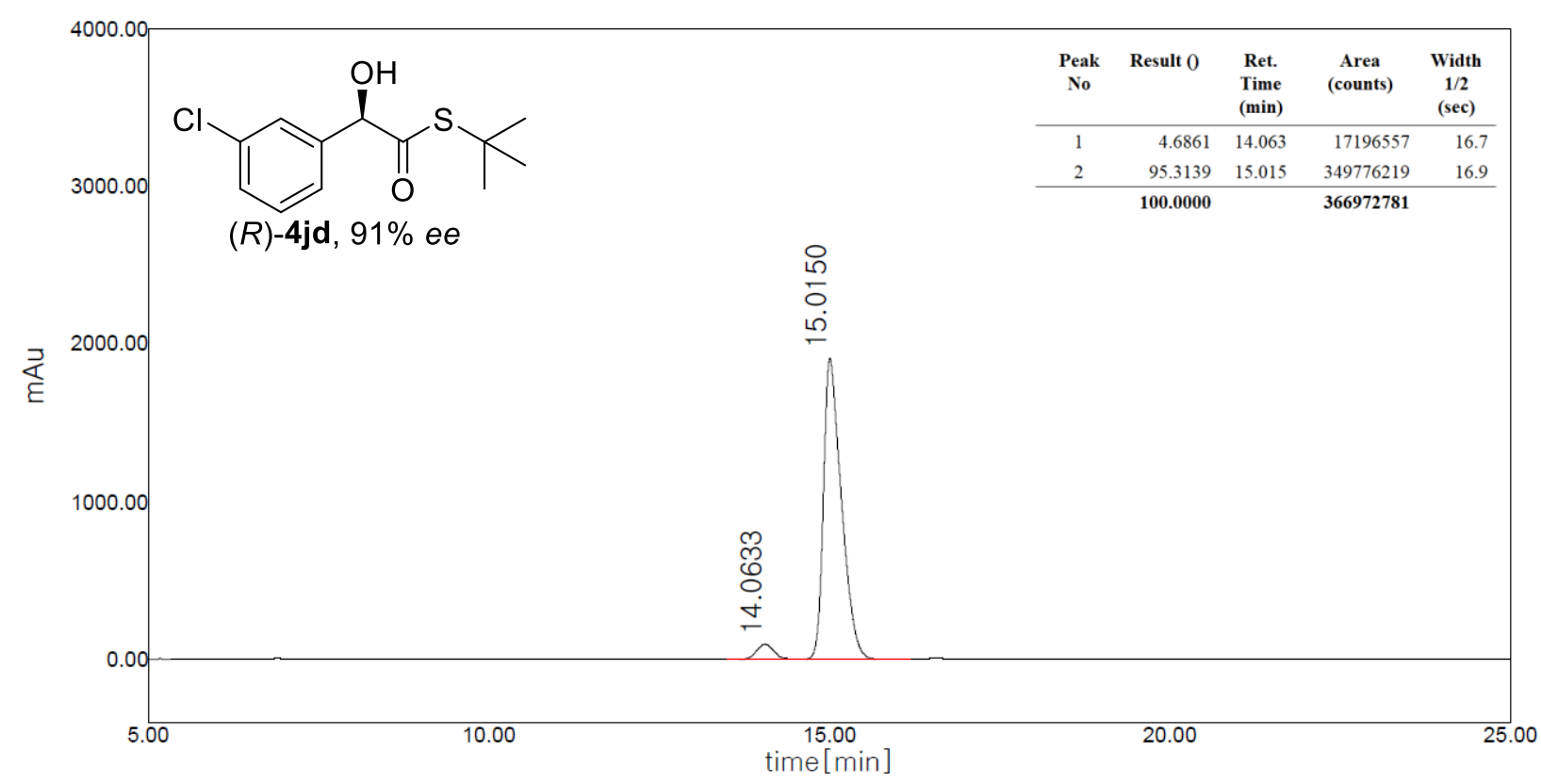

HPLC trace of $\mathbf{4 j d}$ (condition a) 


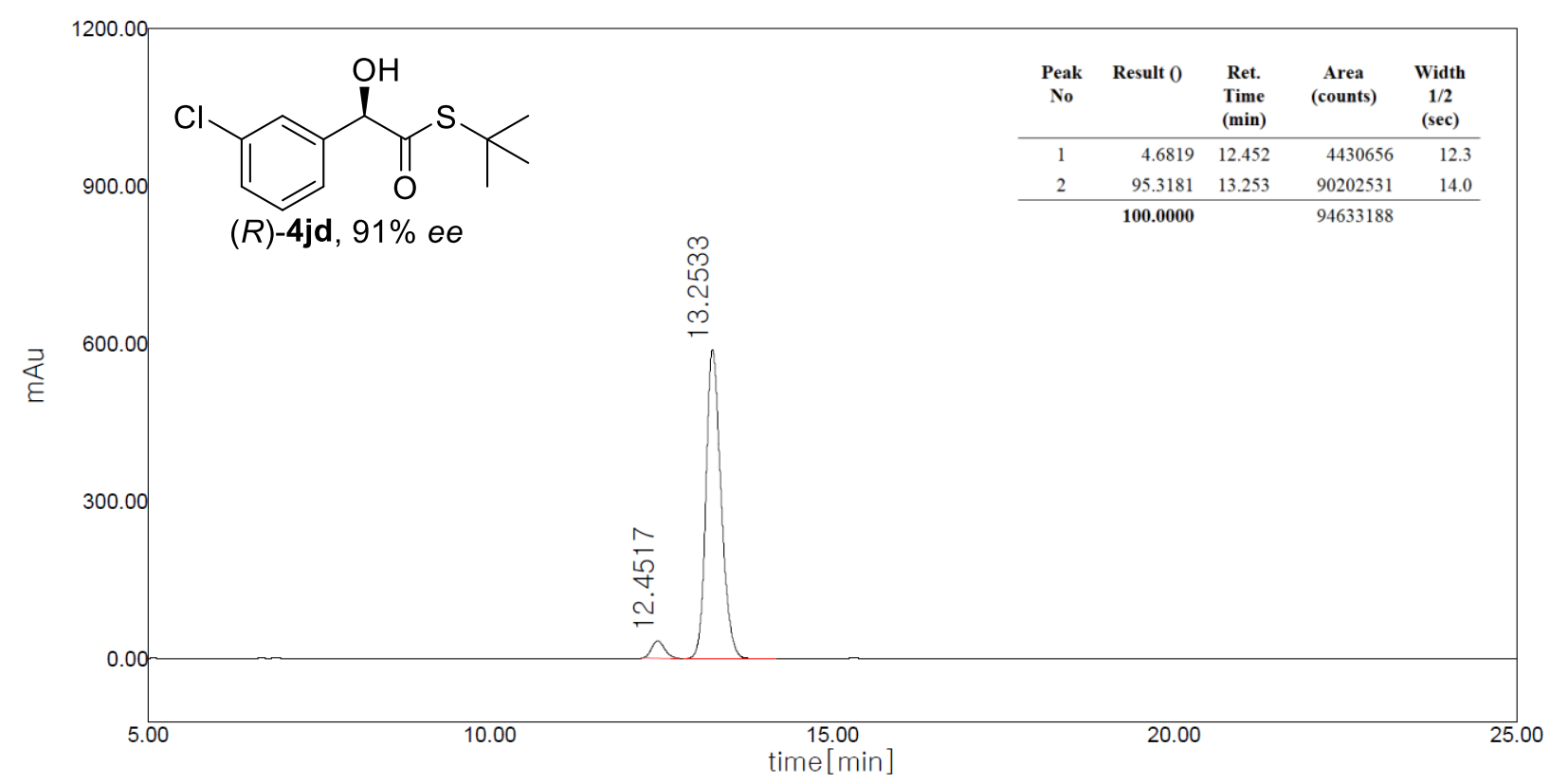

HPLC trace of $\mathbf{4 j d}$ (condition b)

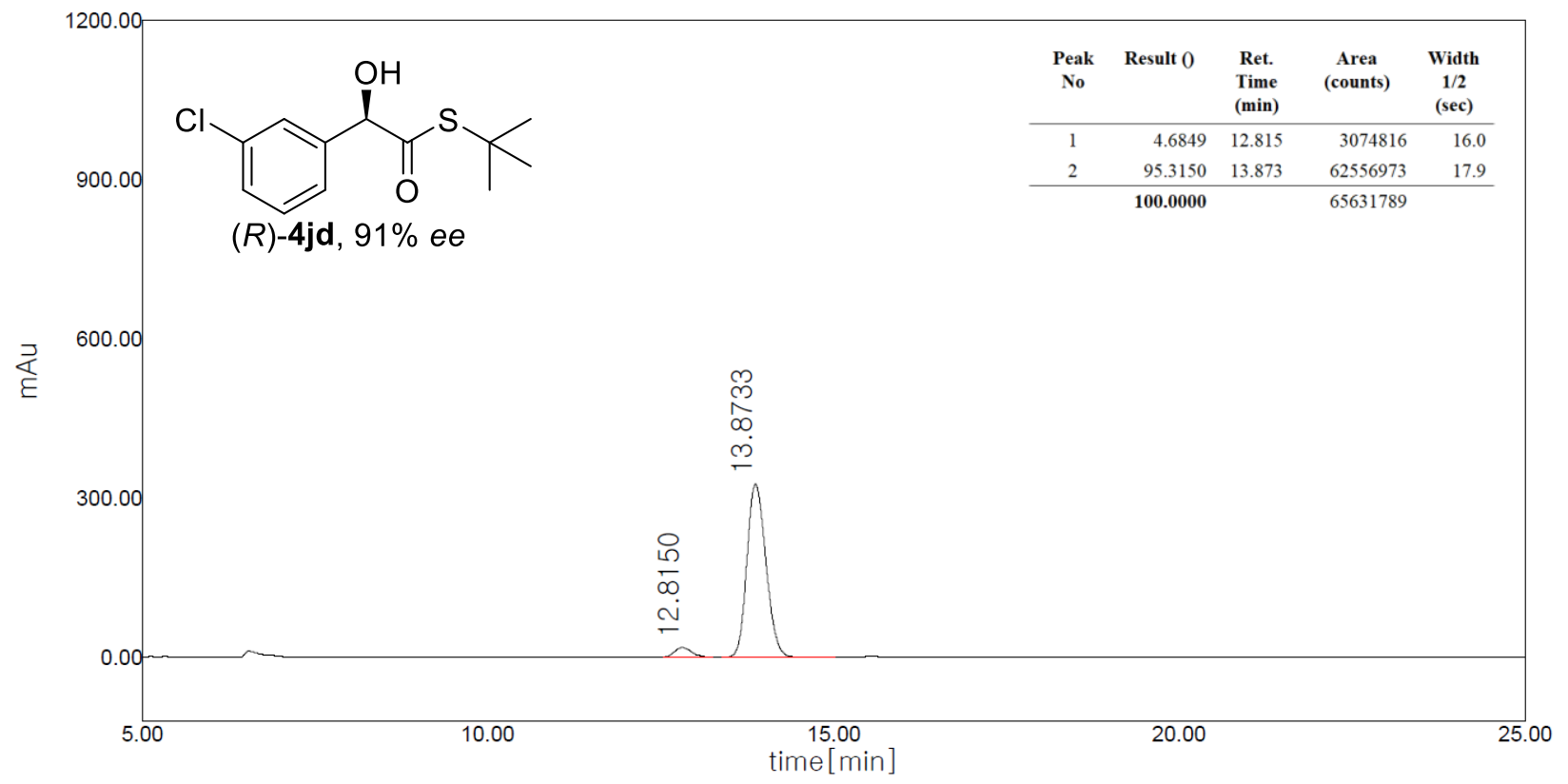

HPLC trace of $\mathbf{4 j d}$ (condition c) 


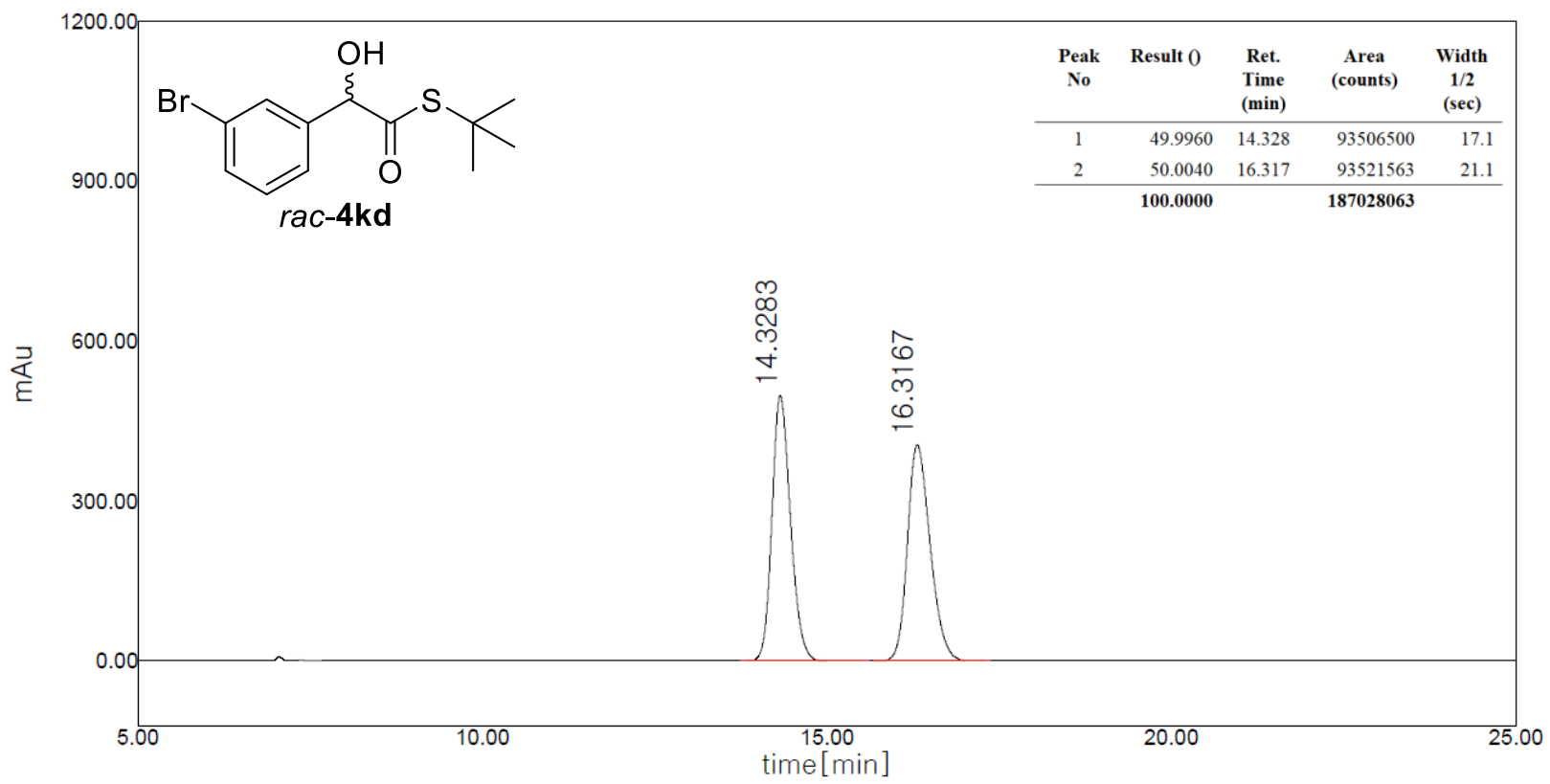

HPLC trace of $r a c-\mathbf{4 k d}$

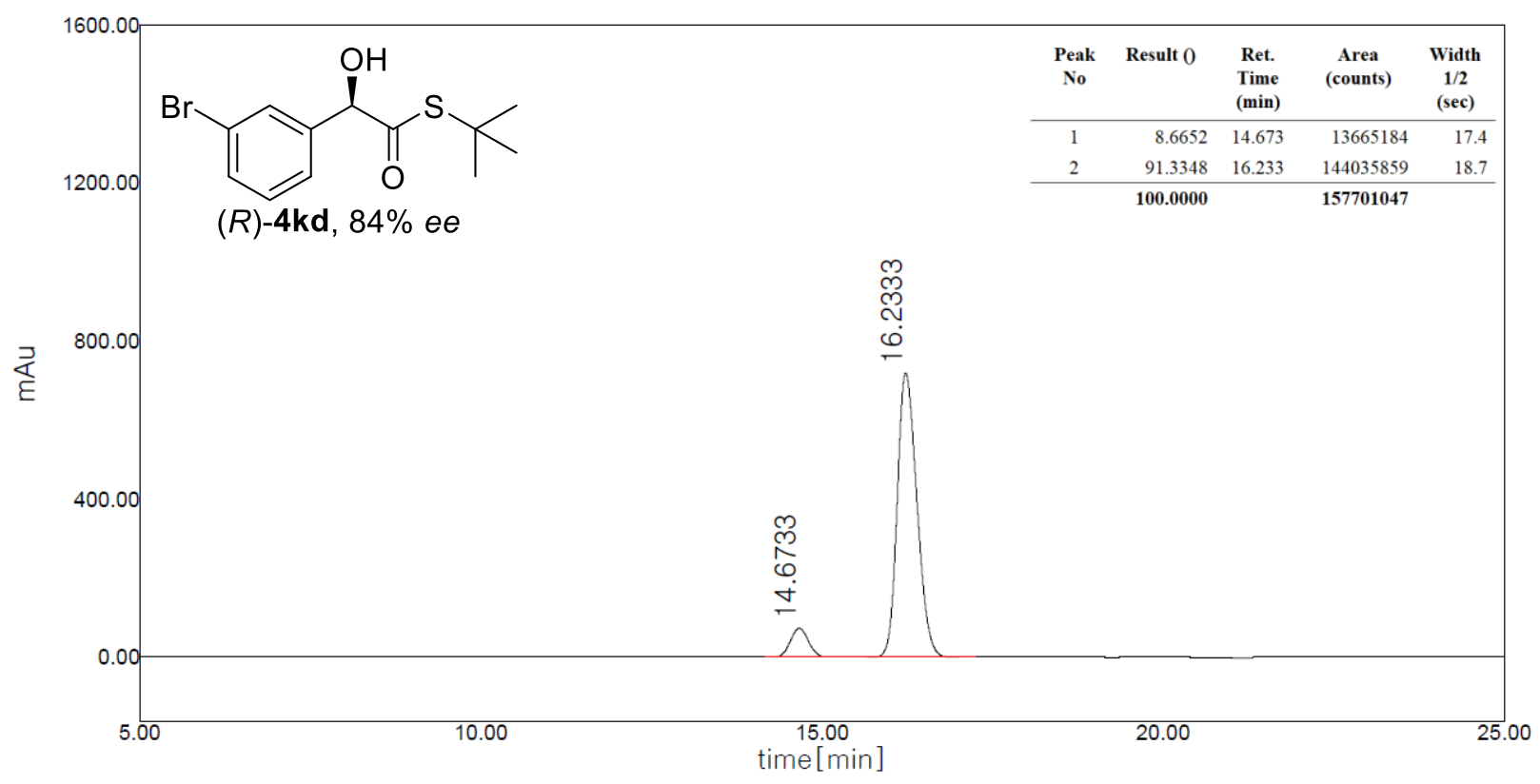

HPLC trace of $\mathbf{4 k d}$ (condition a) 


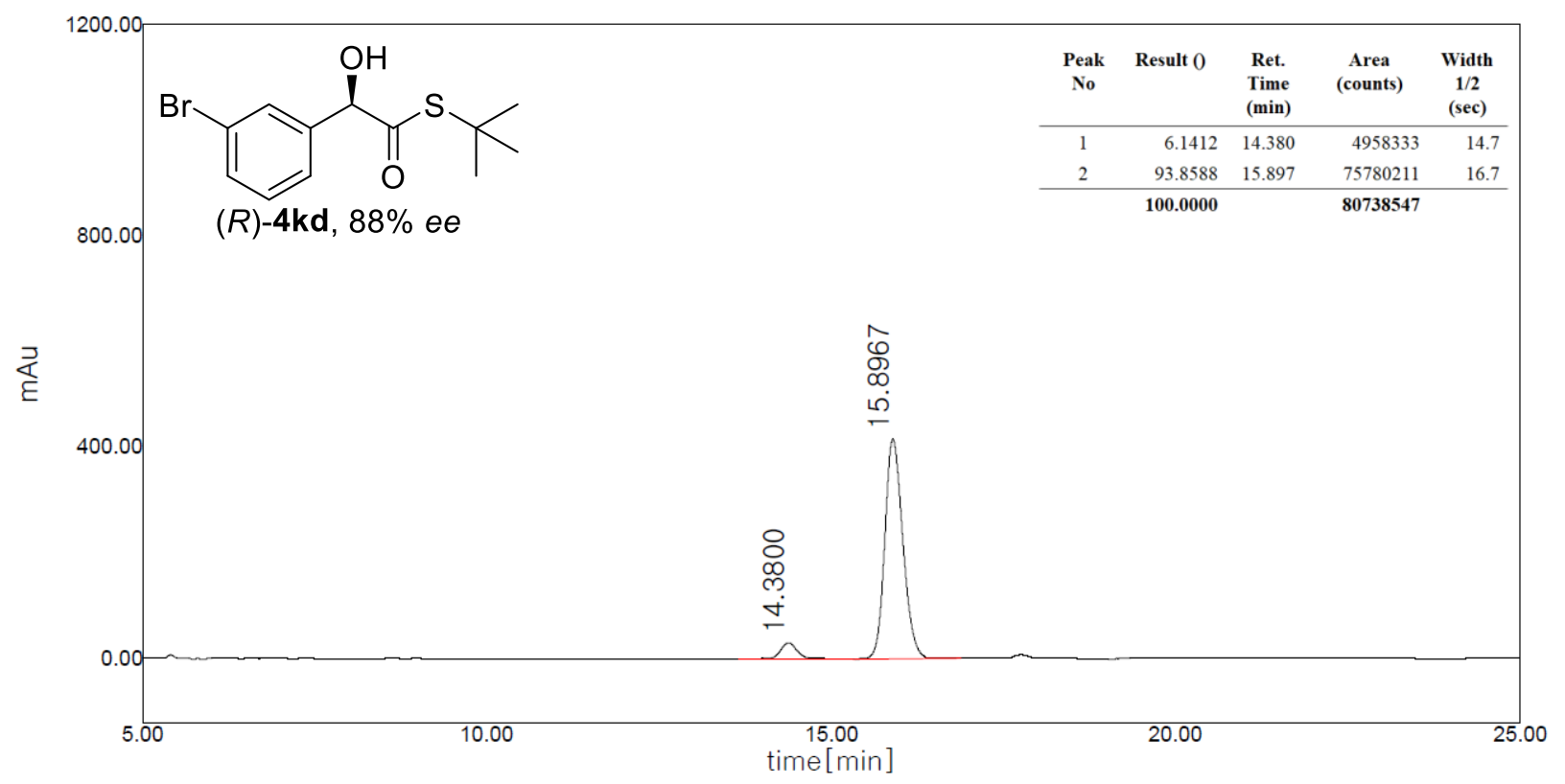

HPLC trace of $\mathbf{4 k d}$ (condition b)

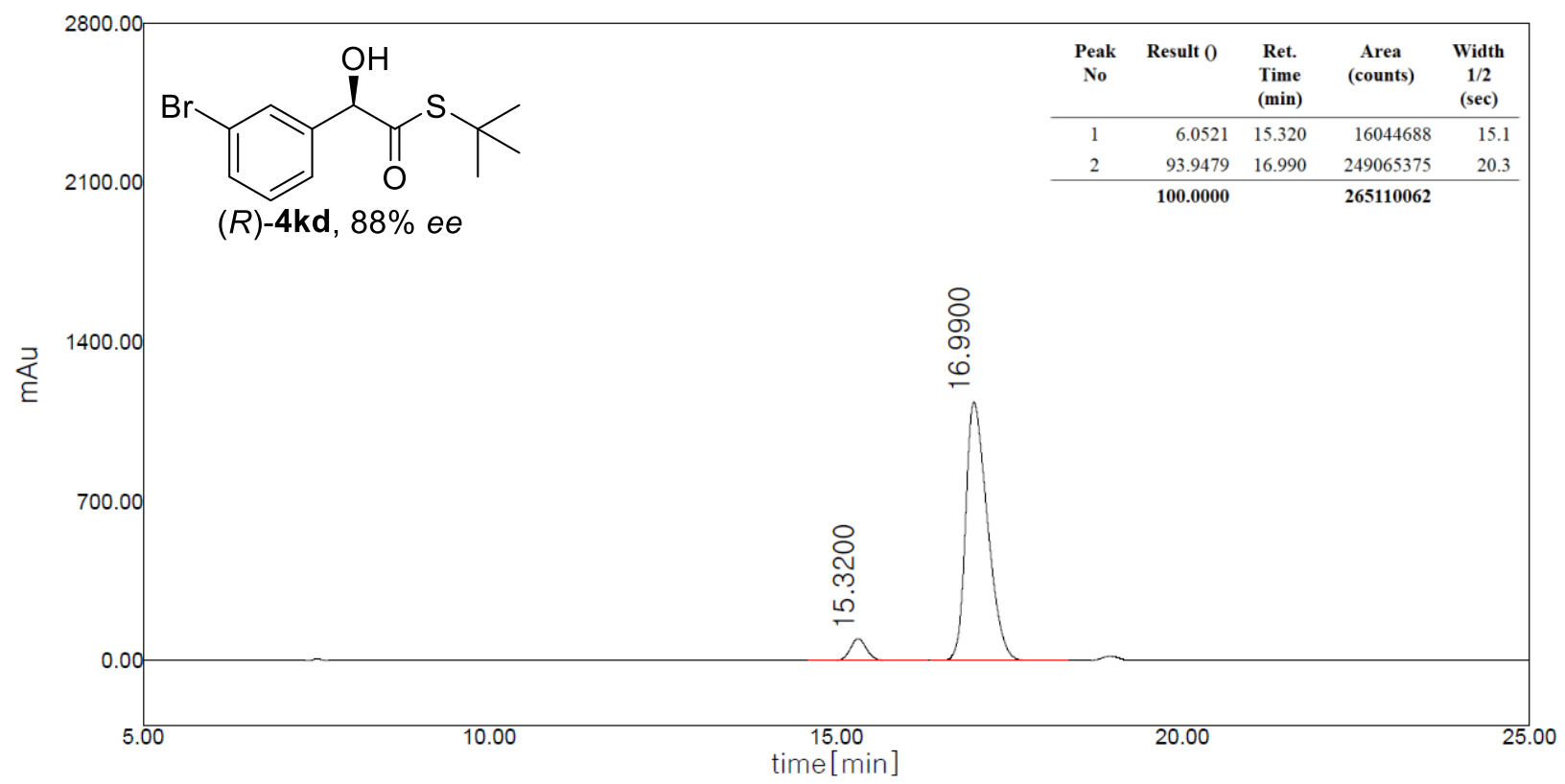

HPLC trace of $\mathbf{4 k d}$ (condition c) 


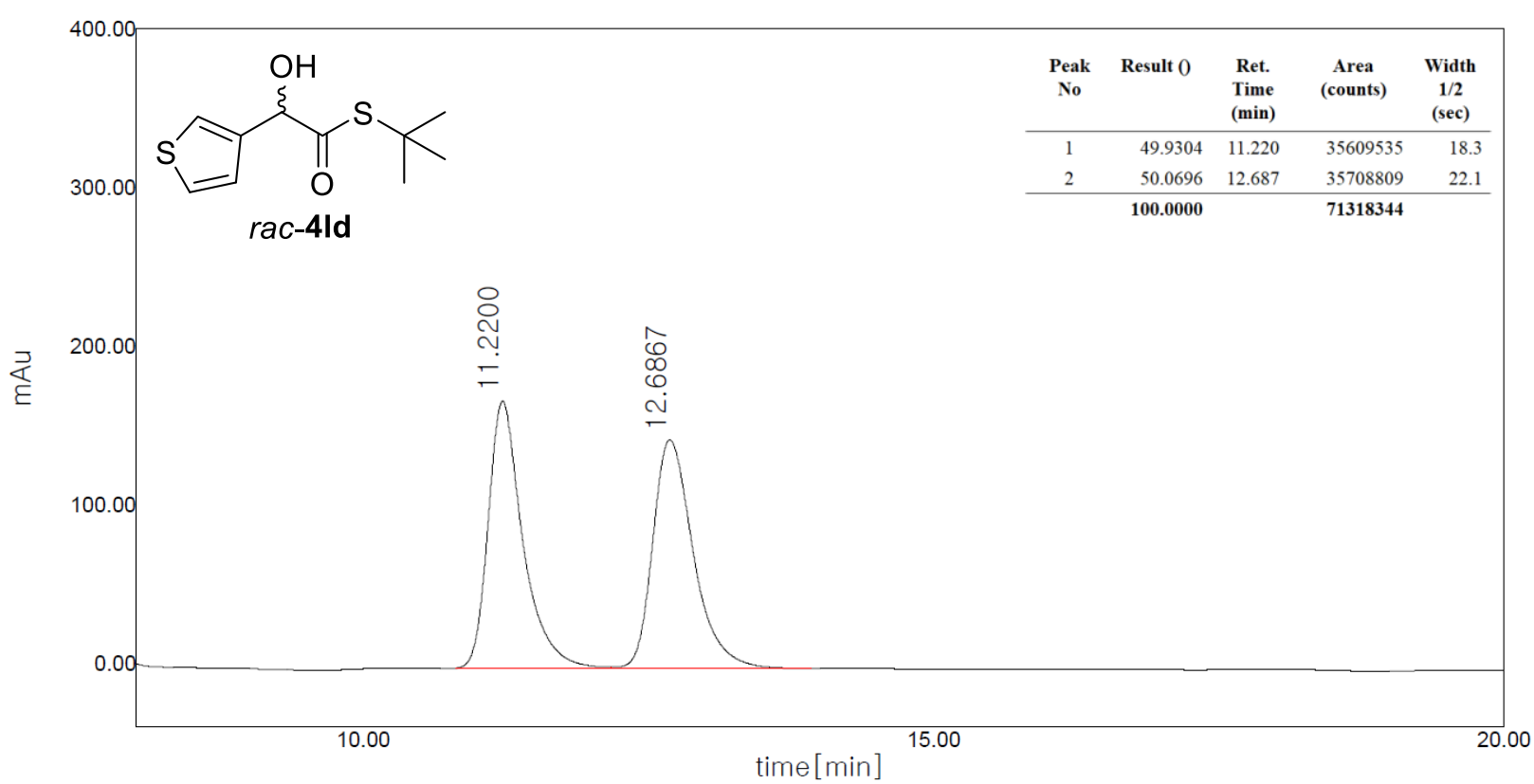

\section{HPLC trace of $r a c-4 l d ~$}

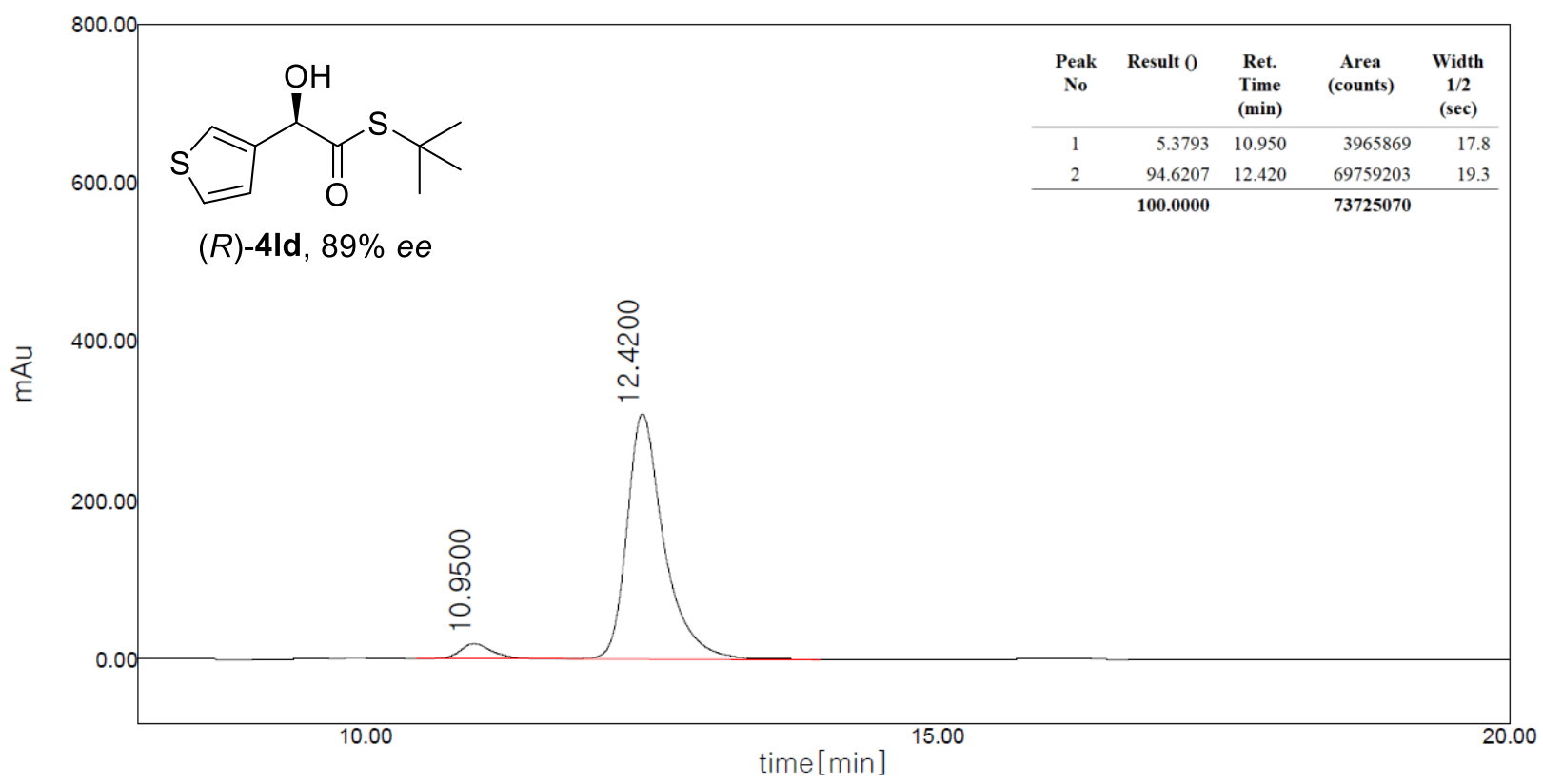

HPLC trace of 4ld (condition a) 


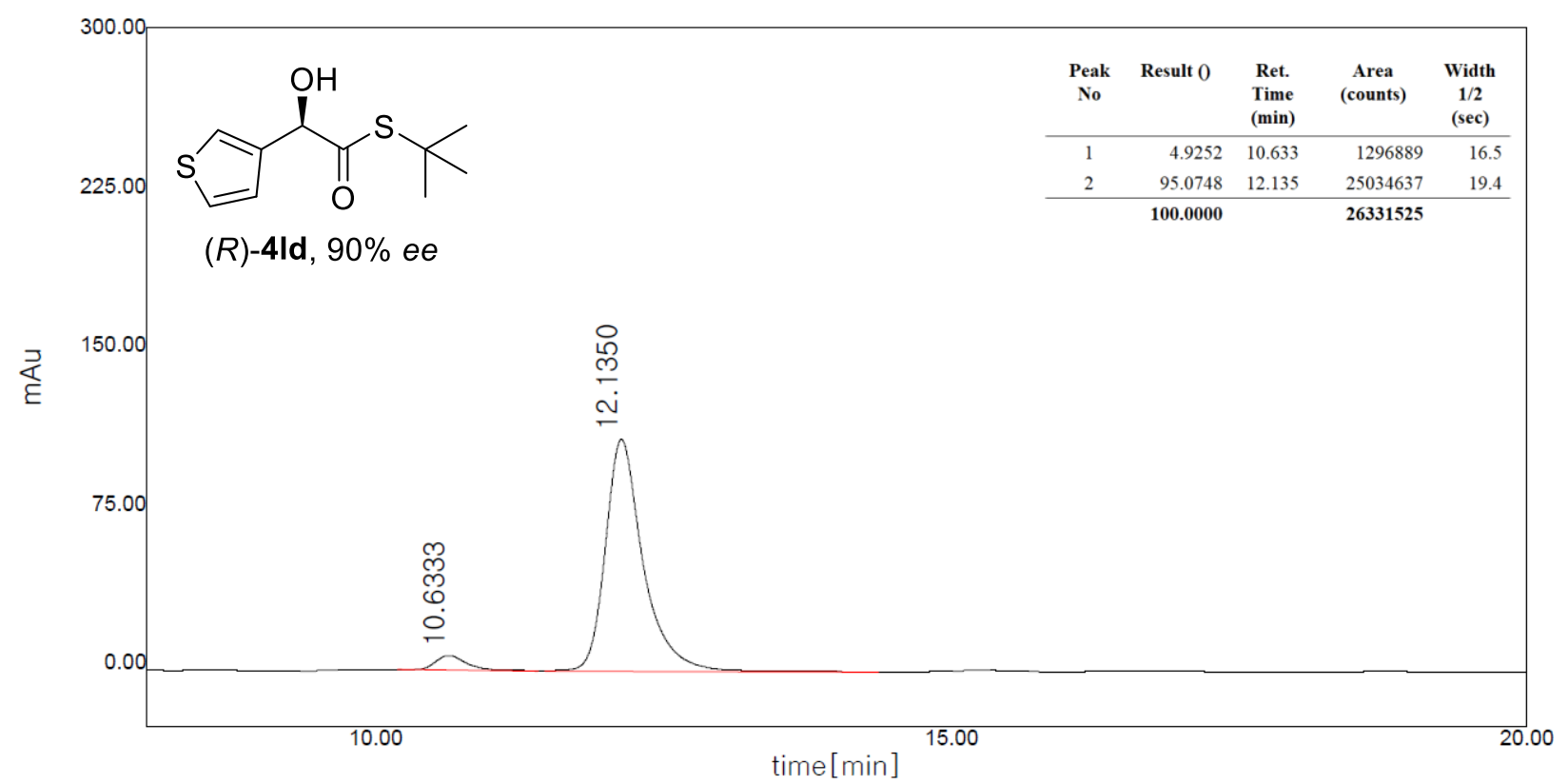

HPLC trace of 4ld (condition b)

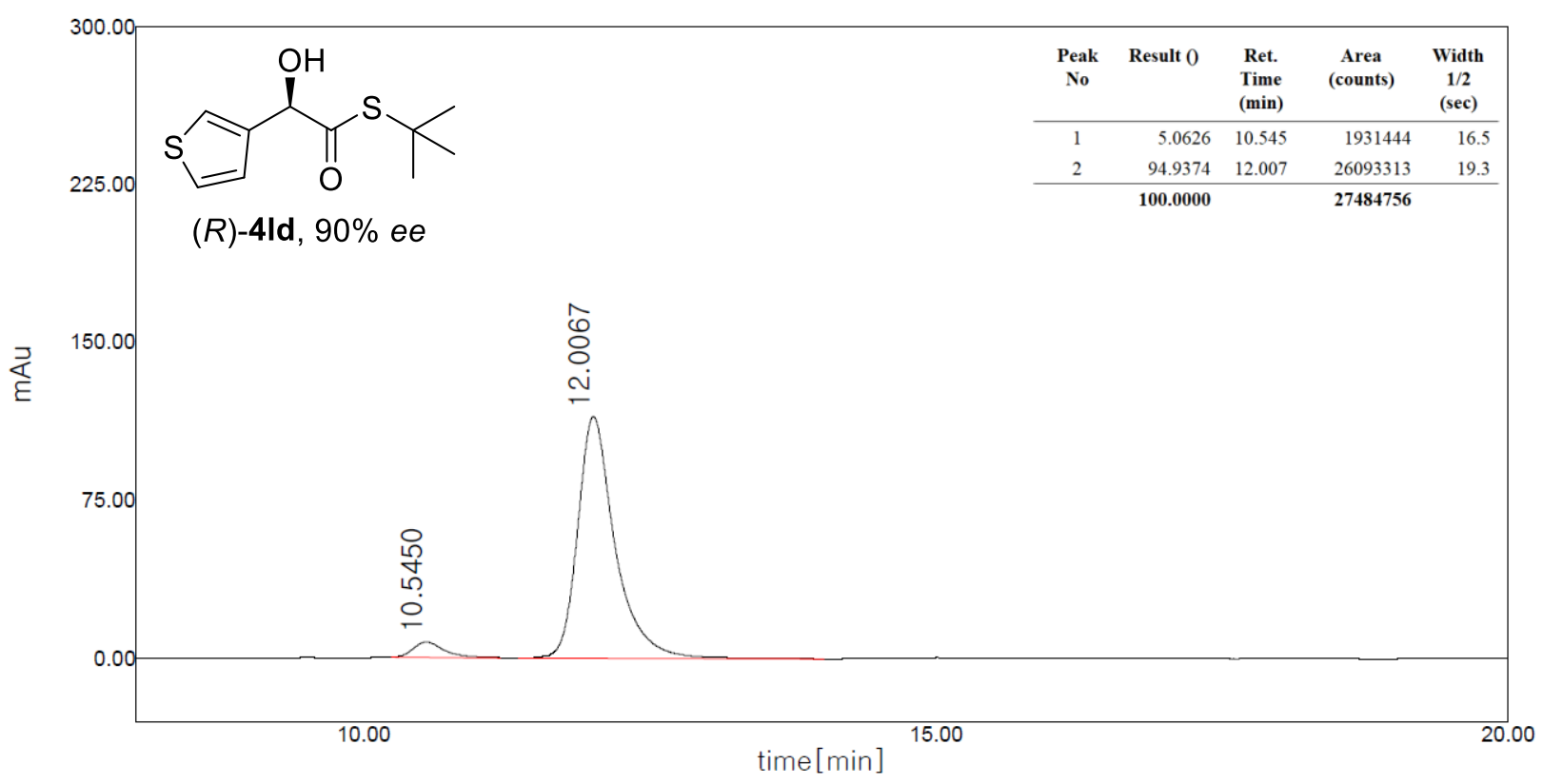

HPLC trace of 4ld (condition c) 


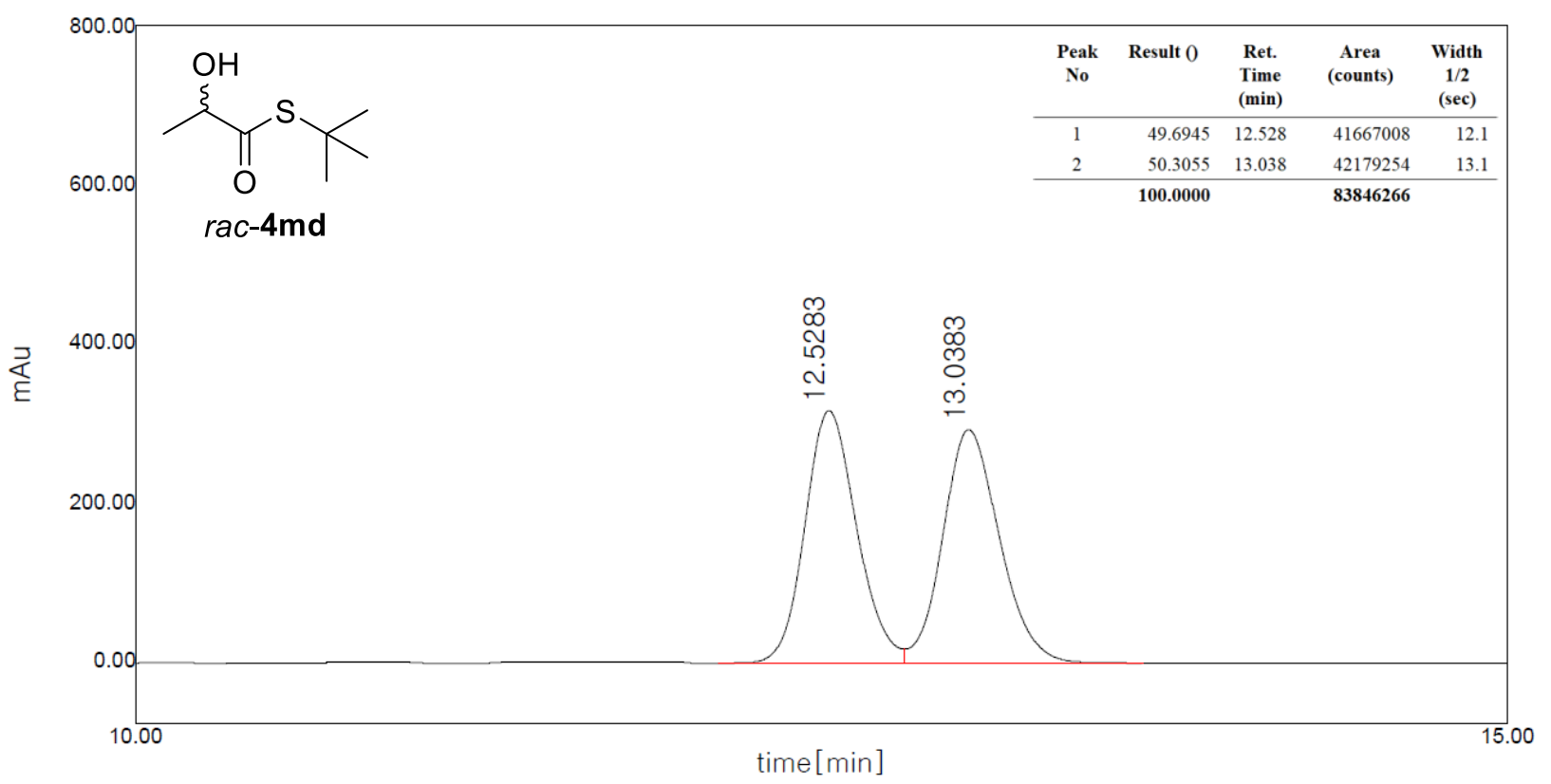

HPLC trace of $r a c-4 m d$

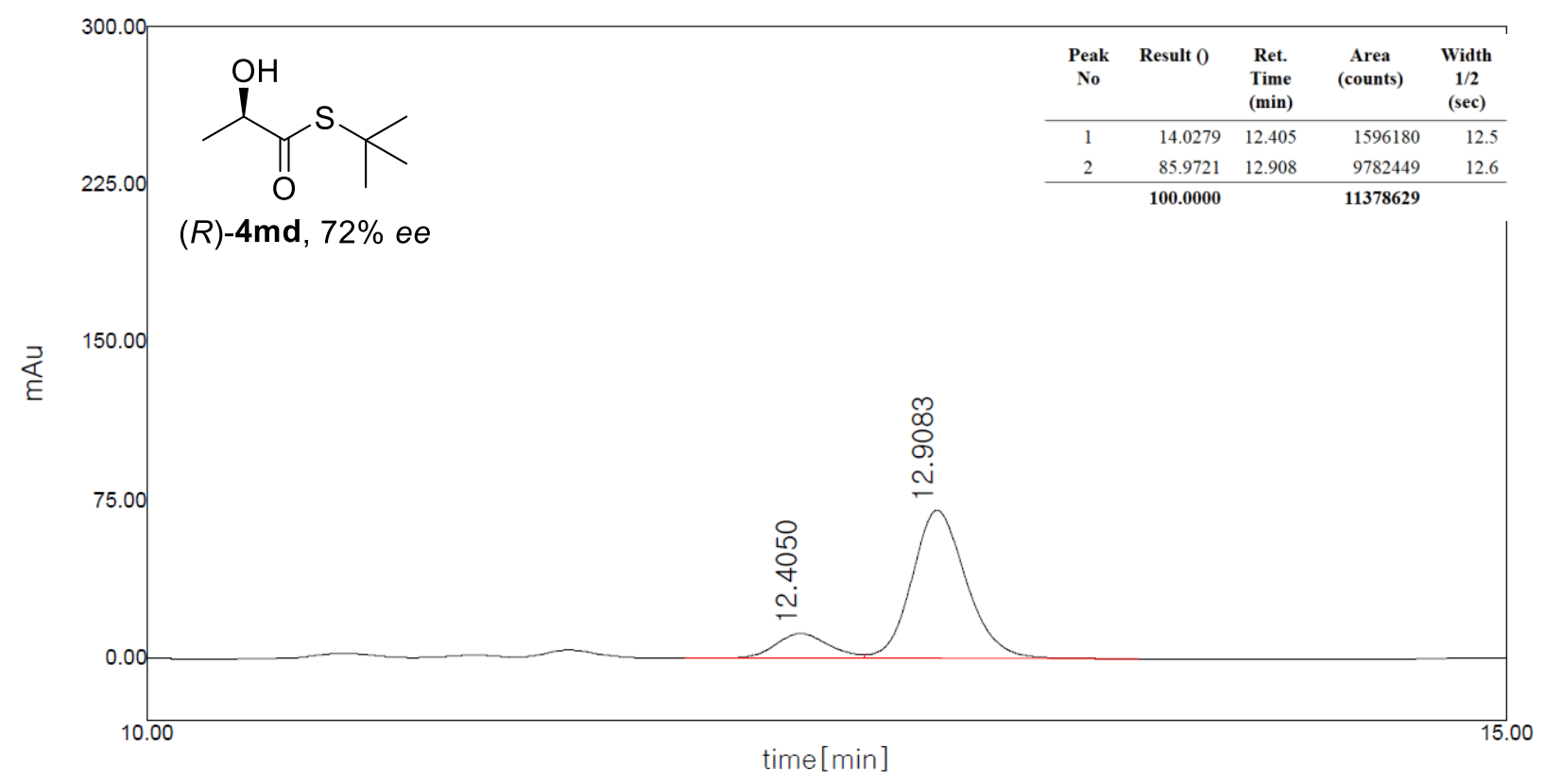

HPLC trace of 4md (condition a) 


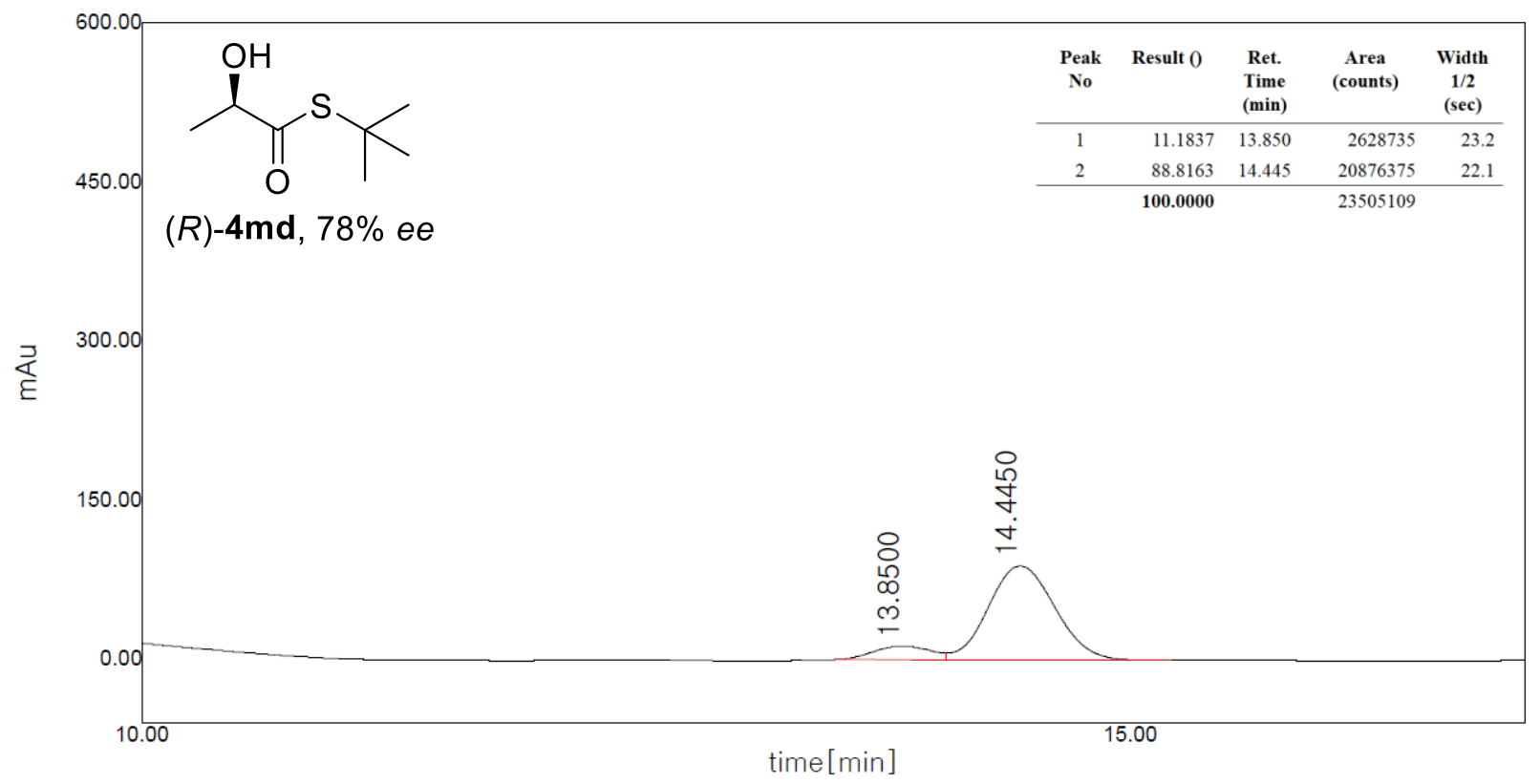

HPLC trace of $\mathbf{4 m d}$ (condition b)

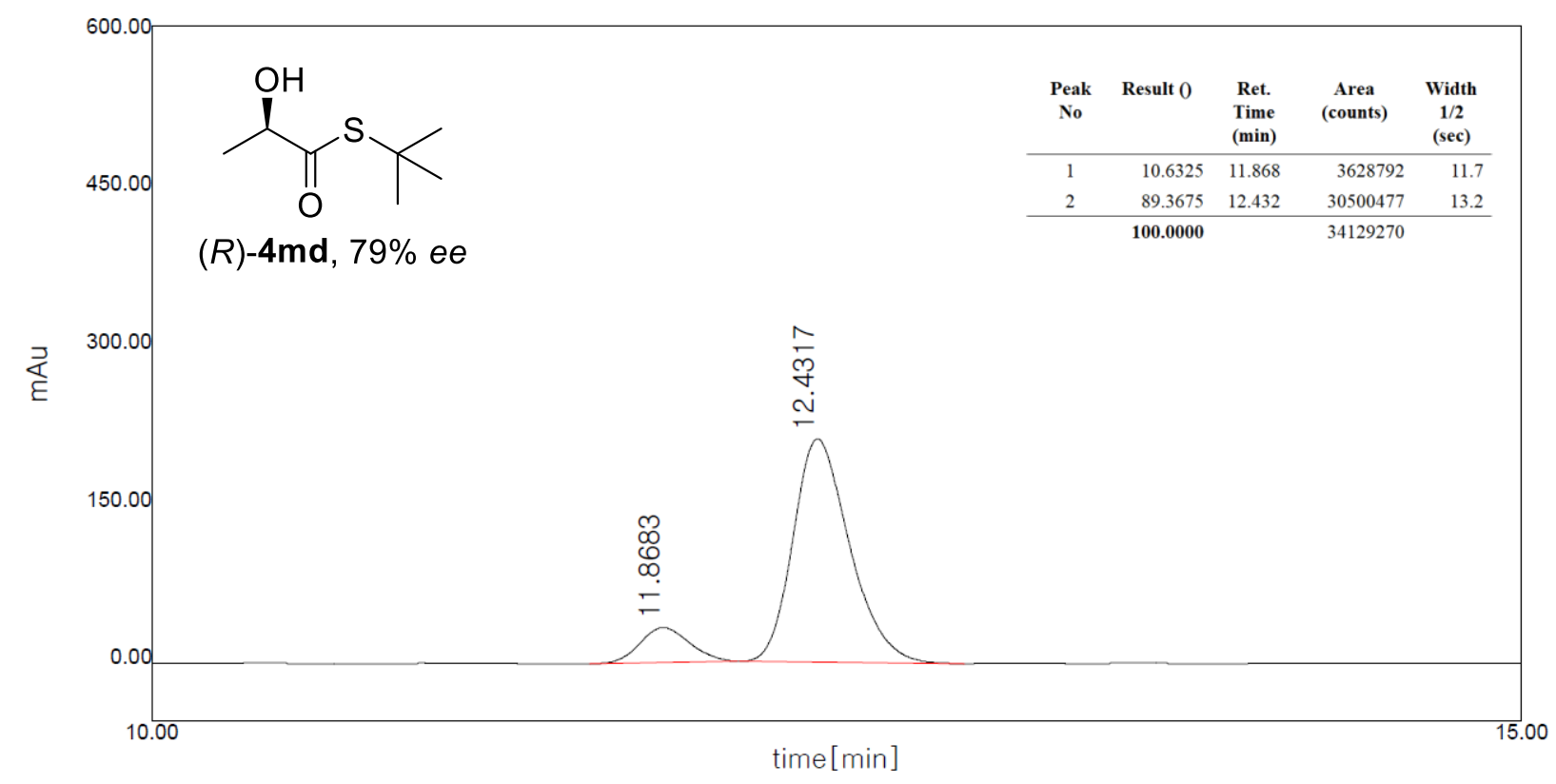

HPLC trace of $\mathbf{4 m d}$ (condition c) 


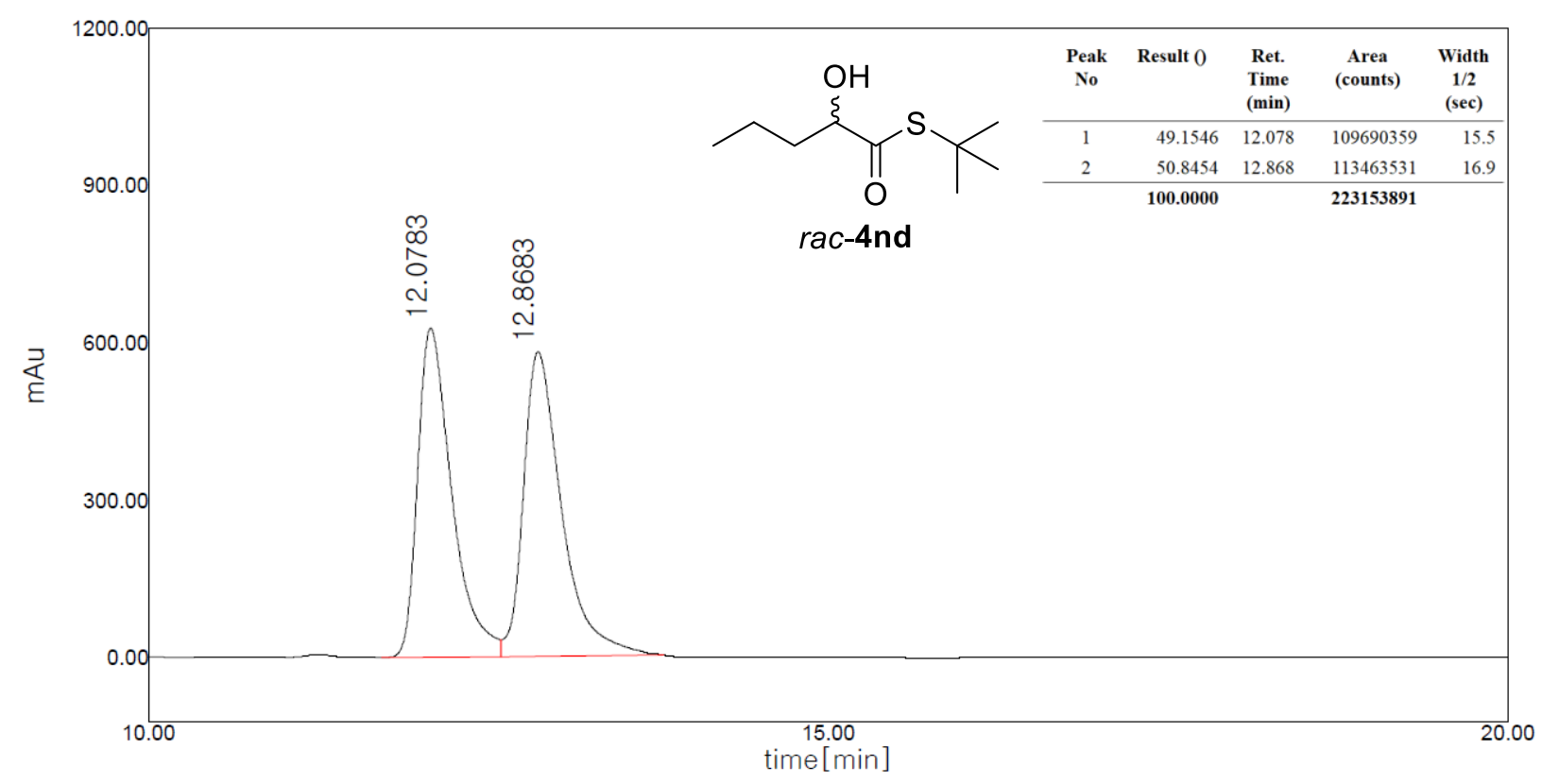

HPLC trace of $r a c-4 n d$

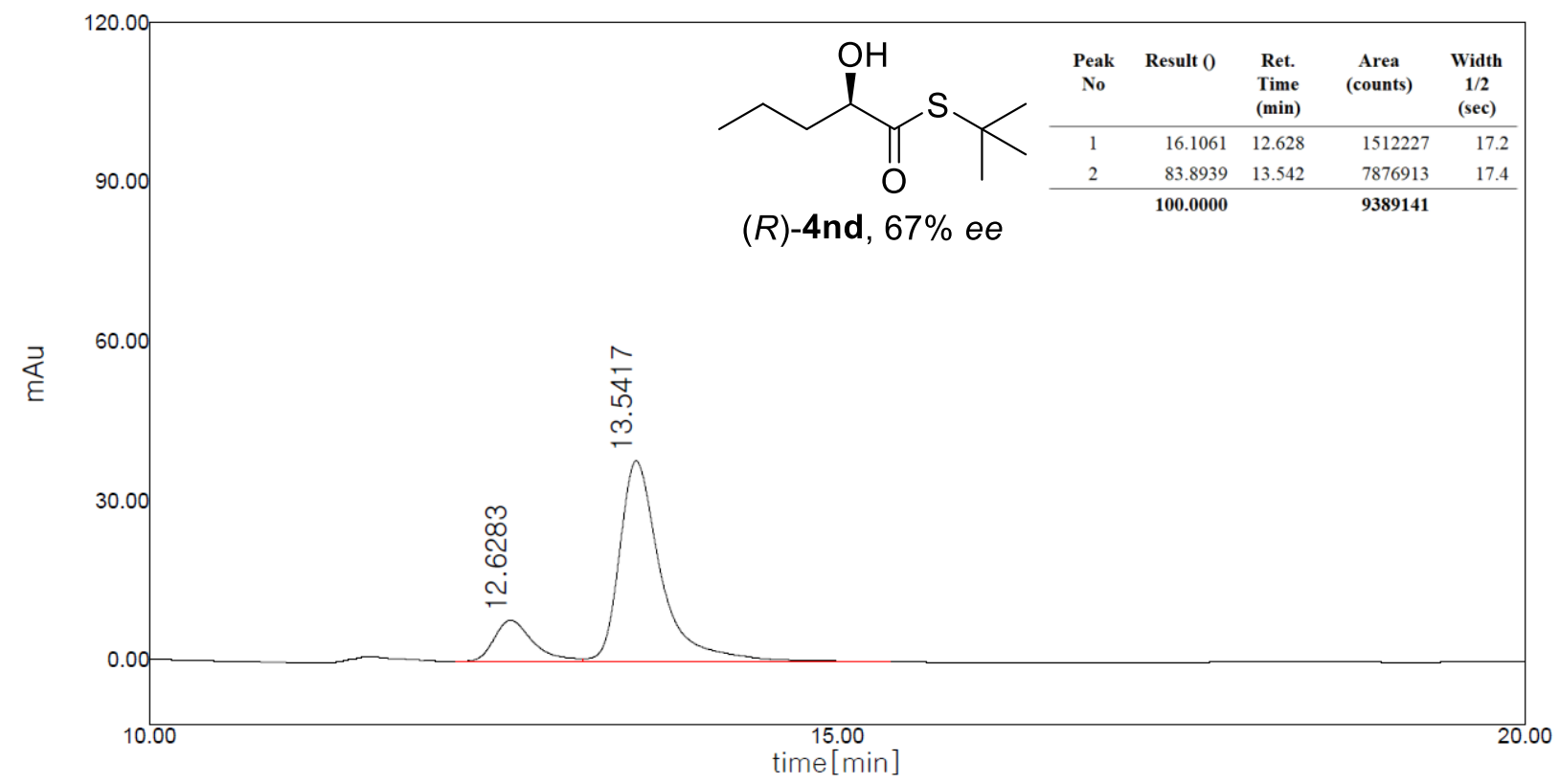

HPLC trace of 4nd (condition a) 


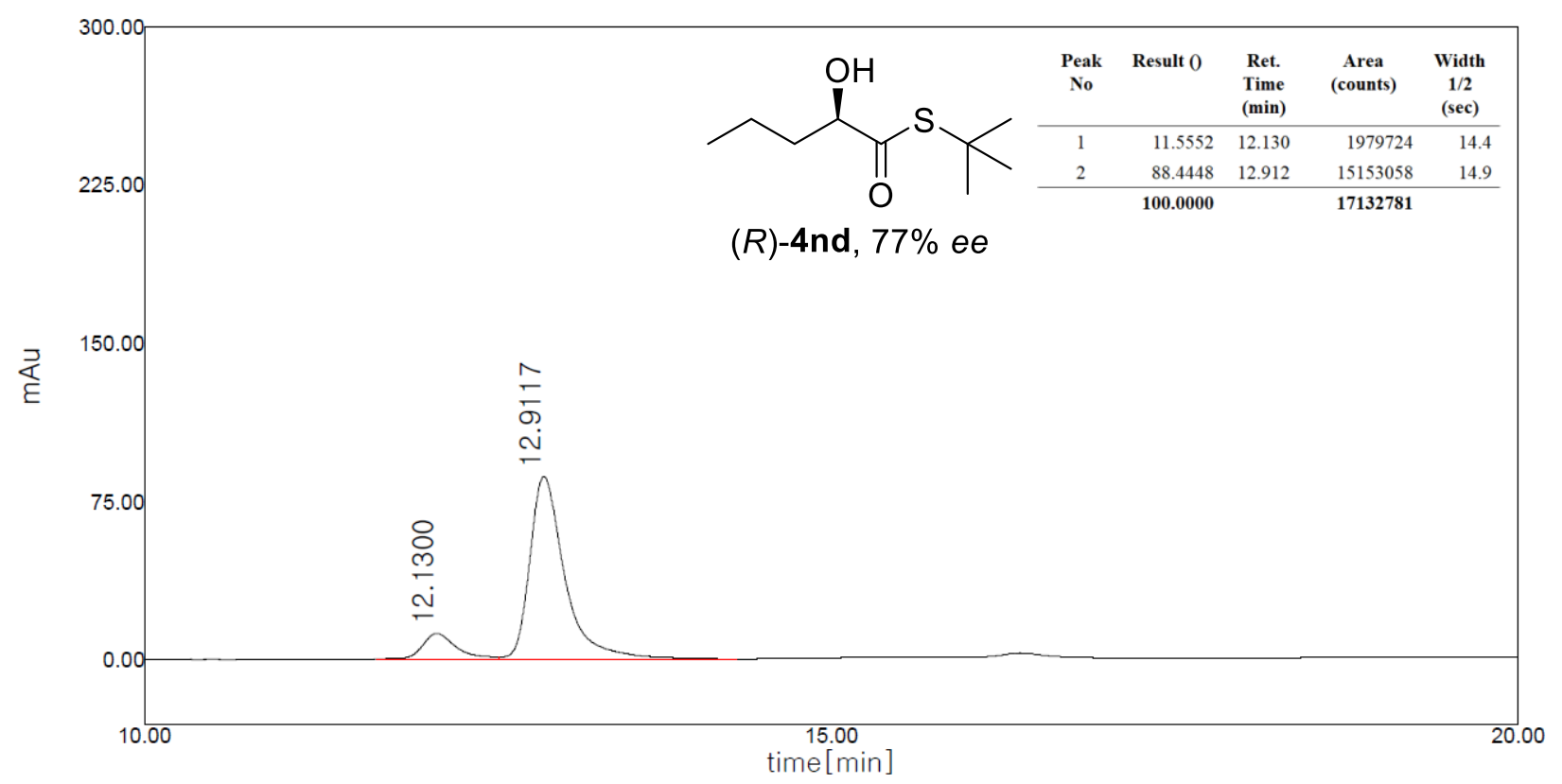

HPLC trace of 4nd (condition b)

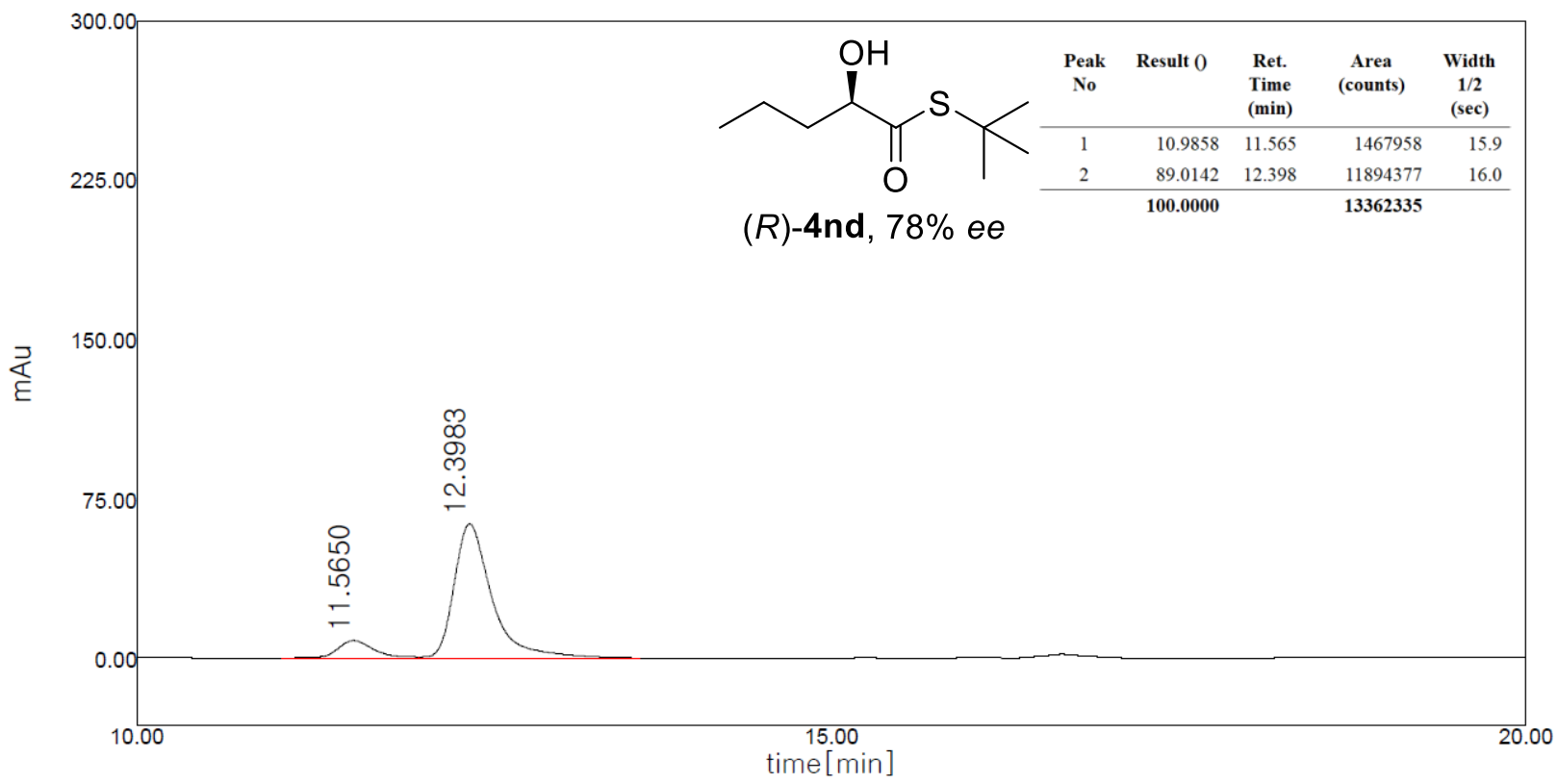

HPLC trace of 4nd (condition c) 


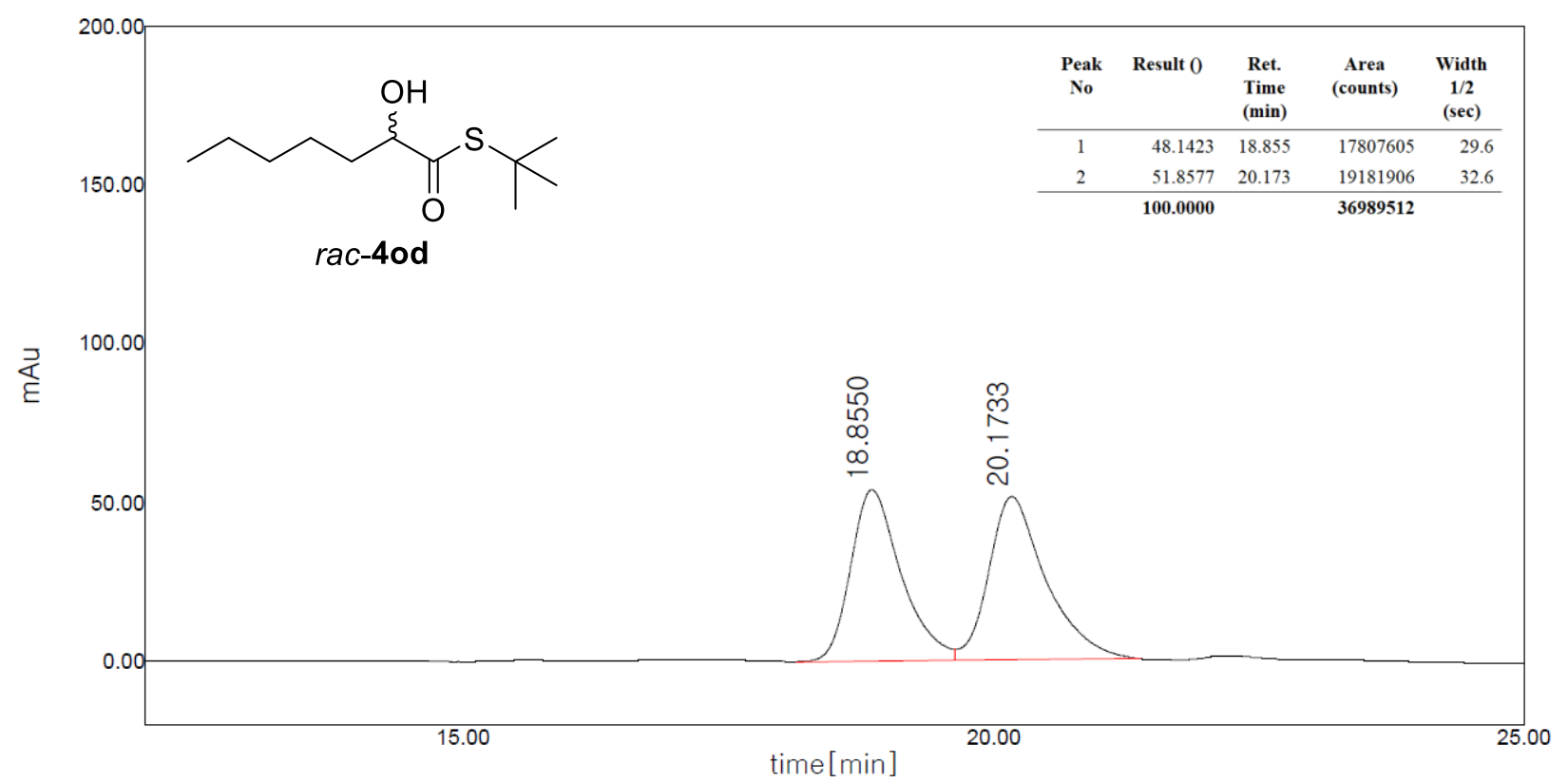

\section{HPLC trace of rac-4od}

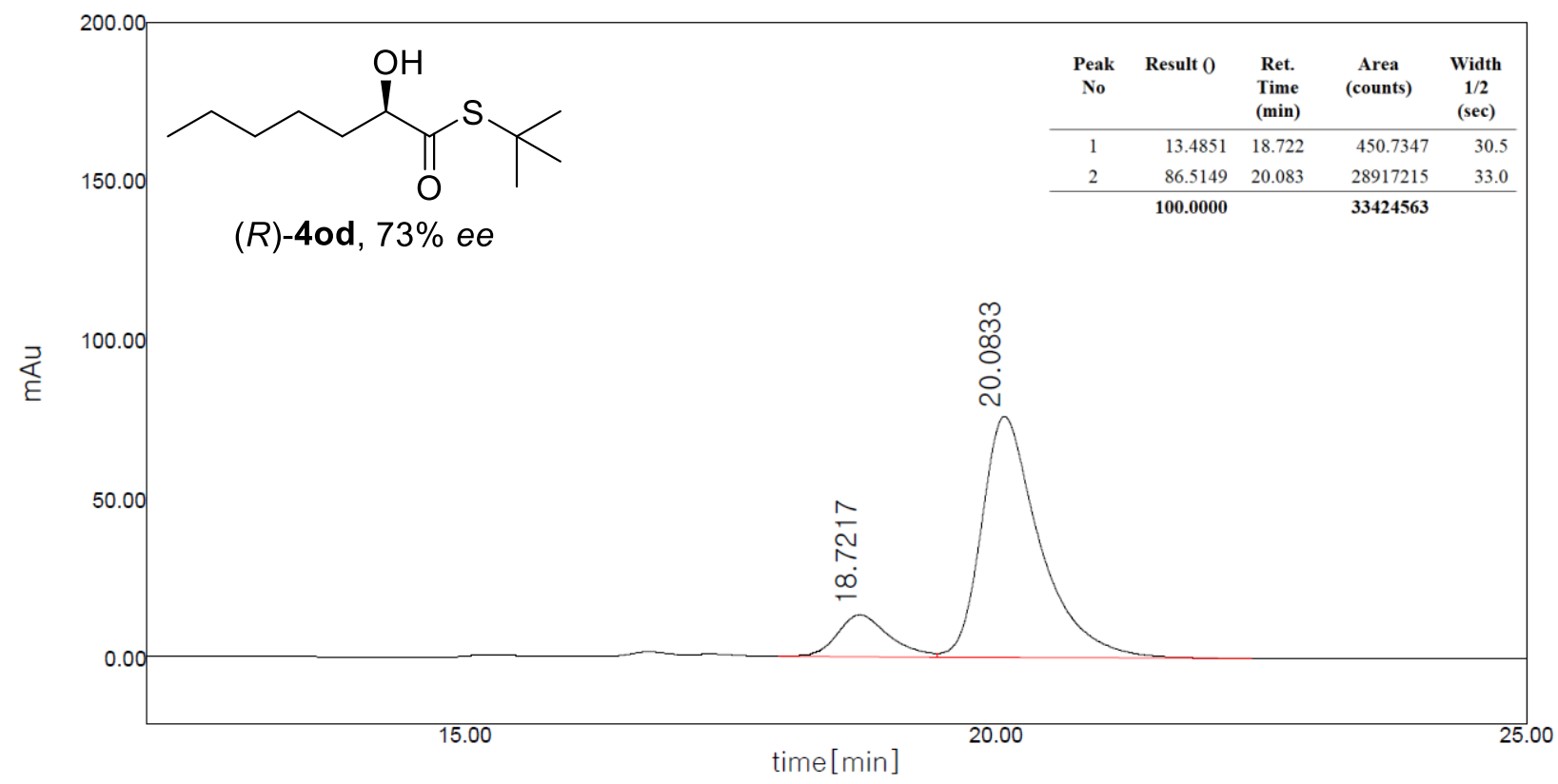

HPLC trace of 4od (condition a) 


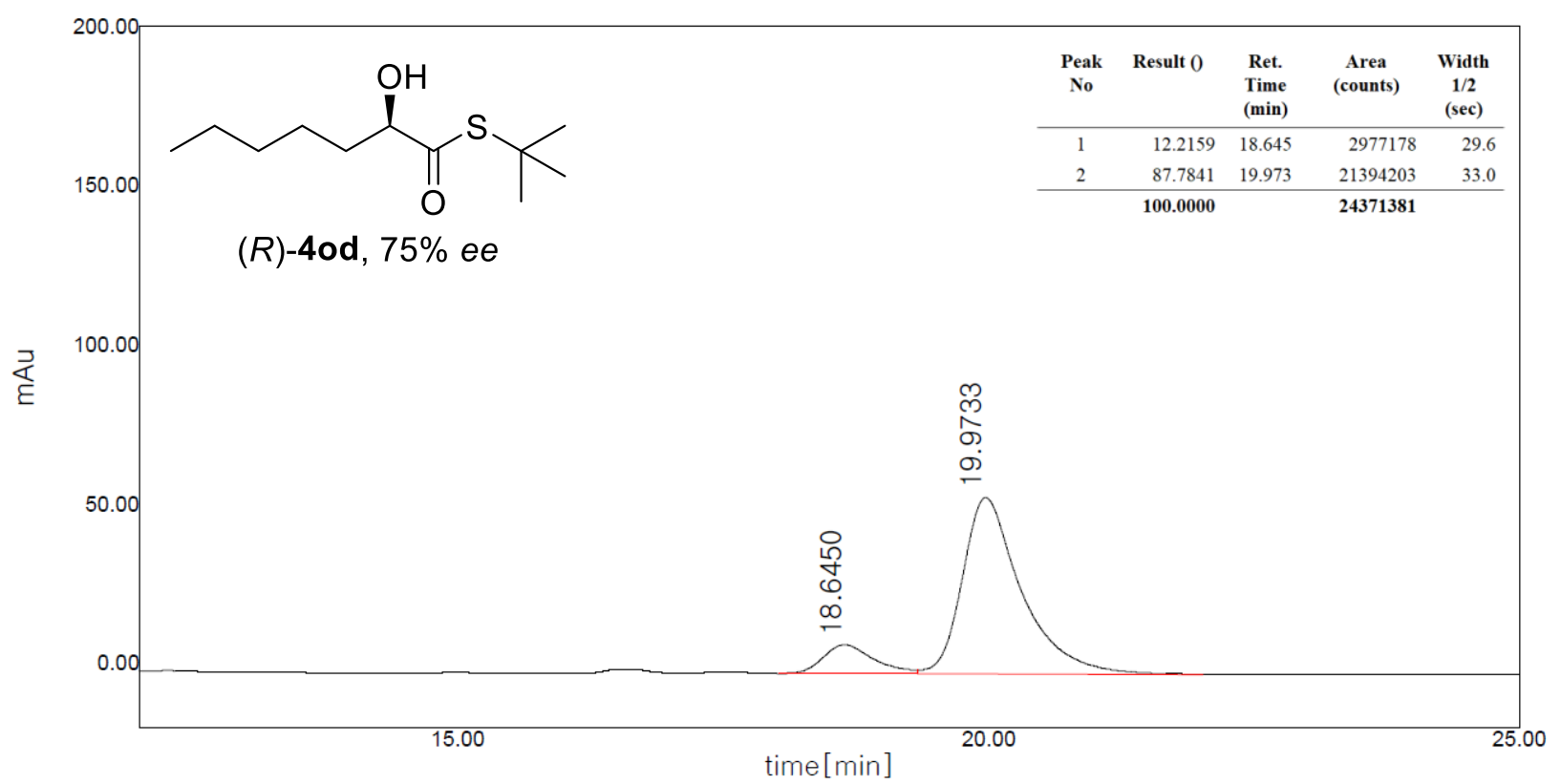

\section{HPLC trace of 4od (condition b)}

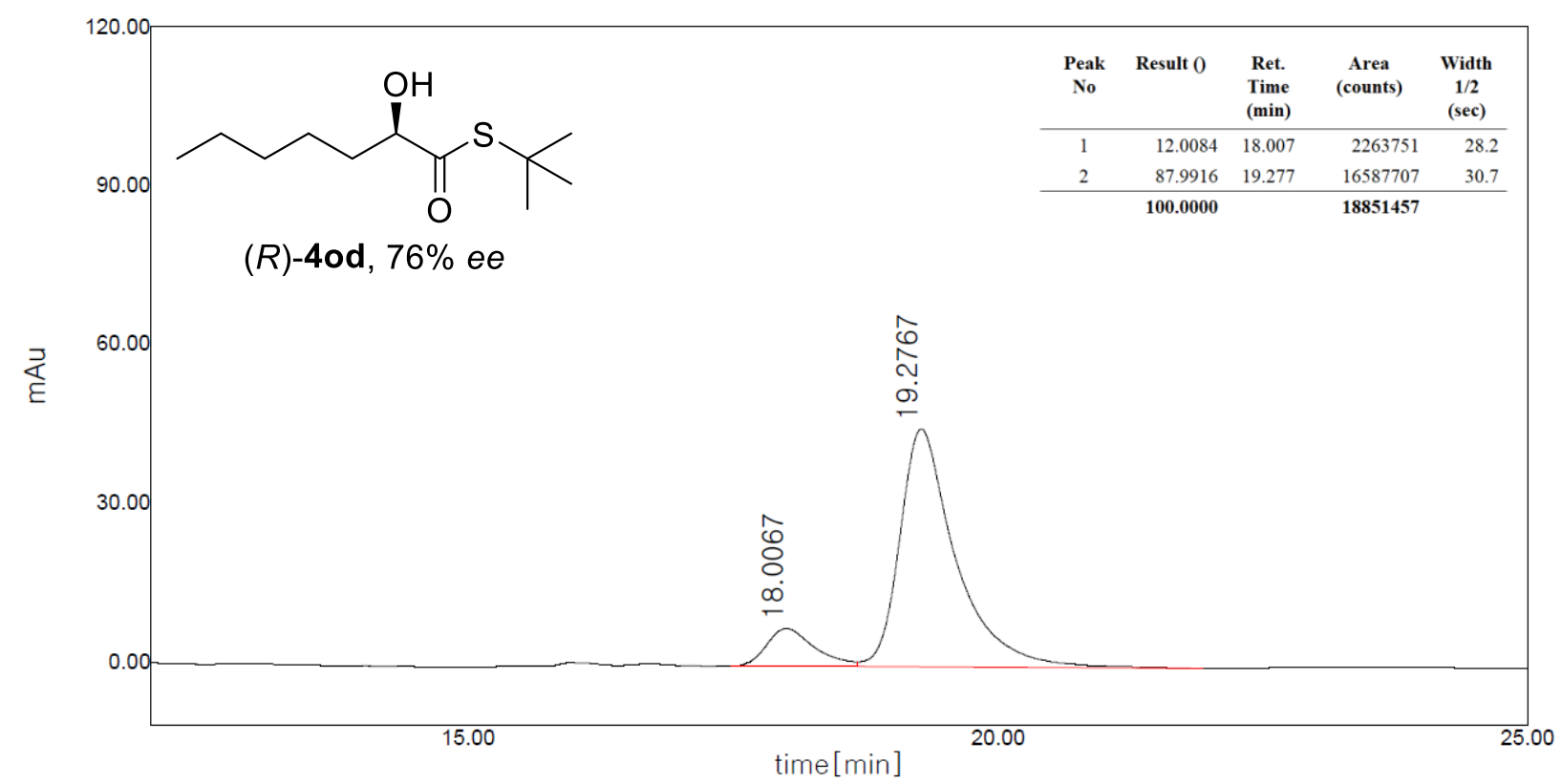

HPLC trace of 4od (condition c) 


\section{References}

1. Riley, H. A.; Gray, A. R. Phenylglyoxal. Org. Synth. 1935, 15, 67.

2. Zheng, Q.; Maksimovic, I.; Upad, A.; Guber, D.; David, Y. Synthesis of an Alkynyl Methylglyoxal Probe to Investigate Nonenzymatic histone Glycation. J. Org. Chem. 2020, 85, 1691-1697.

3. Okino, T.; Yasutaka, H.; Furukawa, T.; Xu, X.; Takemoto, Y. Enantio- and Diastereoselective Michael Reaction of 1,3-Dicarbonyl Compounds to Nitroolefins Catalyzed by a Bifunctional Thiourea. J. Am. Chem. Soc. 2005, 127, 119-125.

4. Park, S. Y.; Hwang, I.-S.; Lee, H.-J.; Song, C. E. Biomimetic catalytic transformation of toxic $\alpha$-oxoaldehydes to high-value chiral $\alpha$-hydroxythioesters using artificial glyoxalase I. Nat. Commun. 2017, 8, 14877.

5. Bergstrom, P. A.; Lindgren, J.; Kristiansson, O. An IR study of the hydration of $\mathrm{ClO}_{4}{ }^{-}, \mathrm{NO}_{3}{ }^{-}$, $\mathrm{I}^{-}, \mathrm{Br}^{-}, \mathrm{Cl}^{-}$, and $\mathrm{SO}_{4}{ }^{2-}$ anions in aqueous-solution. J. Phys. Chem. 1991, 95, 8575-8580. 